\title{
Internal Hydraulic Jumps with Upstream Shear
}

\author{
by \\ Kelly Anne Ogden \\ B.A.Sc., University of Waterloo (2009) \\ M.Math., University of Waterloo (2011) \\ Submitted to the Joint Program in Physical Oceanography \\ in partial fulfillment of the requirements for the degree of \\ Doctor of Philosophy \\ at the \\ MASSACHUSETTS INSTITUTE OF TECHNOLOGY \\ and the \\ WOODS HOLE OCEANOGRAPHIC INSTITUTION
}

February 2017

(c) 2017 Kelly Anne Ogden.

All rights reserved.

The author hereby grants to MIT and WHOI permission to reproduce and to distribute publicly paper and electronic copies of this thesis document in whole or in part in any medium now known or hereafter created.

Author

Joint Program in Physical Oceanography Massachusetts Institute of Technology \& Woods Hole Oceanographic Institution

November 10, 2016

Certified by

Karl Helfrich

Senior Scientist

Woods Hole Oceanographic Institution

Thesis Supervisor

Accepted by

Lawrence J. Pratt

Senior Scientist

Woods Hole Oceanographic Institution

Chair, Joint Committee for Physical Oceanography 


\title{
Internal Hydraulic Jumps with Upstream Shear
}

\author{
by \\ Kelly Anne Ogden
Submitted to the Joint Program in Physical Oceanography
Massachusetts Institute of Technology
\& Woods Hole Oceanographic Institution
on November 10, 2016, in partial fulfillment of the
requirements for the degree of
Doctor of Philosophy

\begin{abstract}
Internal hydraulic jumps in flows with upstream shear are investigated numerically and theoretically. The role of upstream shear has not previously been thoroughly investigated, although it is important in many oceanographic flows such as exchange flows and stratified flow over topography. Several two-layer shock joining theories, characterized by their distribution of dissipation in the jump, are considered and extended to include upstream shear, entrainment, and topography. Theoretical results are also compared to 2D and some 3D numerical simulations of the full Navier-Stokes equations, which allow continuous velocity and density distributions.
\end{abstract}

The solution space of idealized jumps with small upstream shear is identified using twolayer theories, which shows that upstream shear allows larger jumps to form and allows jumps for a larger range of parameters. Numerical simulations reveal several jump structures that can occur in these flows, including an undular bore, a fully turbulent jump, and a smooth front turbulent jump. At low shear, the 2D mixing efficiency is constant across simulations. As shear increases, the basic two-layer theories no longer provide solutions. Numerical simulations show that entrainment becomes significant as the shear increases, and adding entrainment and shape parameters to describe the continuous velocity profiles is required to accurately describe the simulations using two-layered theory. The entrainment depends on the upstream shear and can be predicted with a modified theory. However, use of the theory is limited due to its sensitivity to the value of the shape parameters. The $2 \mathrm{D}$ mixing efficiency also decreases significantly as shear increases. Finally, more realistic 2D and some 3D simulations including topography bridge the gap between the highly idealized simulations and the very realistic work of others. Simulations with topography show additional jump types, including a higher mode jump with a wedge of homogeneous, stagnant fluid similar to a structure seen in Knight Inlet. In all cases, numerical simulations are used to identify trends in the mixing and jumps structures that can occur in internal hydraulic jumps.

Thesis Supervisor: Karl Helfrich

Title: Senior Scientist

Woods Hole Oceanographic Institution 


\section{Acknowledgments}

This work was supported by National Science Foundation grant OCE-1029672, the H. B. Bigelow Chair of the Woods Hole Oceanographic Institution, and the Natural Sciences and Engineering Research Council of Canada.

I would like to thank my advisor, Dr. Karl Helfrich, for all of his guidance, support, and advice. He has been a wonderful advisor, and I am very grateful to have had the opportunity to work with him.

I am grateful for my committee, Dr. Larry Pratt, Dr. Rocky Geyer, Dr. Lou St. Laurent, and Prof. Glenn Flierl for their comments and suggestions on my work. They engaged me in useful and interesting scientific discussions that helped shape this work. I am also grateful for my defense chair, Dr. Amala Mahadevan.

Thanks to Dr. Jesús Pineda for sharing his Stellwagen Bank data with me and allowing me to include it in my thesis.

Thanks to my Professors at WHOI and MIT, and to the other students in PO, particularly those with whom I studied for generals and those a year ahead of me who gave advice about program milestones. 


\section{Contents}

1 Introduction $\quad 19$

1.1 Hydraulic Jumps in the Environment . . . . . . . . . . . . . . . . 20

1.2 Previous Findings and Open Questions . . . . . . . . . . . . 30

1.3 Outline of Thesis . . . . . . . . . . . . . . . . 34

2 Internal Hydraulic Jumps with Low Upstream Shear 37

2.1 Introduction . . . . . . . . . . . . . . . . . 37

2.2 Two-layer theories . . . . . . . . . . . . . . . . . . . 40

2.2.1 Solution space of basic two-layer theories . . . . . . . . . . 43

2.3 Numerical modeling . . . . . . . . . . . . . . . . . . 47

2.3.1 Solution types . . . . . . . . . . . . . . . 54

2.3.2 Numerical simulations compared to layer theories . . . . . . 58

2.3.3 Solitary waves solutions . . . . . . . . . . . . . 66

2.4 Mixing . . . . . . . . . . . . . . . . . . . . . 69

2.5 Three-dimensional simulations . . . . . . . . . . . . . . . 71

2.6 Conclusions . . . . . . . . . . . . . . . . . 75

3 Internal Hydraulic Jumps with High Upstream Shear 79

3.1 Introduction to high shear jumps . . . . . . . . . . . . . 80

3.2 Two-layer theory with entrainment and shape parameters . . . . . . . 82

3.3 Numerical simulations . . . . . . . . . . . . . . . . . . . 92

3.3.1 Simulation results . . . . . . . . . . . . . . . . . 95

3.3.2 Shape parameters and continuous profiles . . . . . . . . 103

3.3.3 Jump structure . . . . . . . . . . . . . . . . . . . 108 
3.3 .4 Vorticity budget. . . . . . . . . . . . . . . . . . 113

3.4 Predicting entrainment . . . . . . . . . . . . . . . . . . . . . 114

3.4.1 Wilkinson and Wood Approach . . . . . . . . . . . 124

3.5 Varying Weir Parameters . . . . . . . . . . . . . . . . . . . 130

3.6 Theory effectiveness . . . . . . . . . . . . . . . . . . . . . . . . . . . . 134

3.7 Trends in mixing efficiency and entrainment . . . . . . . . . . . . 134

3.8 Comparison with 3D $\ldots \ldots \ldots \ldots \ldots \ldots$

3.9 Conclusions . . . . . . . . . . . . . . . . . . . . . . . . . . . . 141

4 Flow over topography $\quad 145$

4.1 Introduction . . . . . . . . . . . . . . . . . . . 145

4.2 Two-layer theory with topography . . . . . . . . . . . . . 146

4.2.1 Theory with instantaneous step . . . . . . . . . . . . . . 147

4.2 .2 Numerical simulations . . . . . . . . . . . . . . . . . . . 151

4.2 .3 Theory compared to simulations . . . . . . . . . . . . . 153

4.3 Simulation of more realistic flows . . . . . . . . . . . . 157

4.3 .1 Simulation setup . . . . . . . . . . . . . . 158

4.3.2 Parameter space of realistic flows . . . . . . . . . . . . 159

4.3 .3 Flow structure . . . . . . . . . . . . . . . . . . . 161

4.3.4 Mass budgets . . . . . . . . . . . . . . . . . 171

4.3 .5 Mixing . . . . . . . . . . . . . . . . . . . 179

4.3.6 Simulations with viscosity . . . . . . . . . . . . 187

4.3.7 3D Simulations . . . . . . . . . . . . . . . . . . . 198

4.4 Stellwagen Bank . . . . . . . . . . . . . . . . . . . . 202

4.5 Conclusions of jumps with topography _ . . . . . . . . 207

5 Conclusions 211

5.1 Theory . . . . . . . . . . . . . . . . . . . . . . 211

5.2 Simulations: jump types . . . . . . . . . . . . . . . . . 214

5.3 Simulations: mixing . . . . . . . . . . . . . . . . . . 215

5.4 Applications . . . . . . . . . . . . . . . . . 216

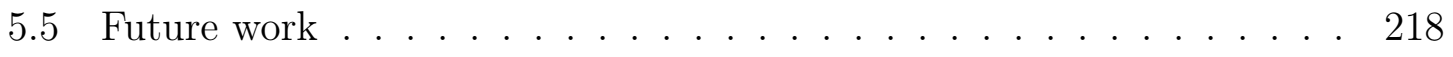


A Calculating Entrainment with Controlled Flow at Weir

A.1 Lower Layer . . . . . . . . . . . . . . . . . . . . . . . . . . 222

A.2 Upper layer . . . . . . . . . . . . . . . . . . . . . . 224

A.3 Combining upper and lower layer equations to find $h_{1 e} \ldots \ldots \ldots 227$

A.4 Combining upper and lower layer equations to find $h_{1 b}$, knowing $h_{1 e}$. 228

B Taylor-Goldstein equation and solution

231 


\section{List of Figures}

1-1 Knight Inlet location and topography . . . . . . . . . . . . . . . 21

1-2 Knight Inlet hydraulic jump . . . . . . . . . . . . . . . . . . 23

1-3 Hood Canal location . . . . . . . . . . . . . . . 24

1-4 Strait of Gibraltar hydraulic jump . . . . . . . . . . . . . . 26

1-5 Pre-Bosphorus Channel hydraulic jump . . . . . . . . . . . . . 27

1-6 Stellwagen Bank location and topogarphy . . . . . . . . . . . . 28

2-1 Idealized problem setup. . . . . . . . . . . . . . 40

2-2 Full and allowable solution spaces. . . . . . . . . . . . . . 45

2-3 Allowable solution space at various shear values. . . . . . . . . . 45

2-4 Length scales of the flow initialization . . . . . . . . . 50

2-5 Numerical simulation showing jump development in time. . . . . . 53

2-6 Jump types at low shear. . . . . . . . . . . . . . 56

2-7 Regime diagram of jump types throughout parameter space. . . . . . 56

2-8 Instantaneous density fields for simulations with negative upstream shear. 58

2-9 Numerical simulations compated to theoretical solution space for $s=1$

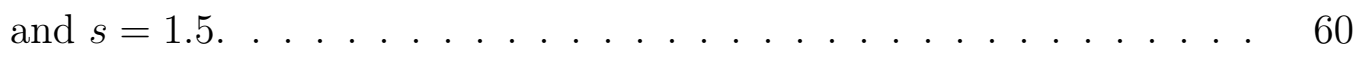

2-10 Density and velocity profiles of downstream flow. . . . . . . . . 61

2-11 Energy flux difference along streamlines versus downstream height of streamline. . . . . . . . . . . . . . . 63

2-12 Vorticity budgets for smooth front turbulent jump and fully turbulent jump. ........................... 66

2-13 Bore front speed compared to DJL predictions. . . . . . . . . . 68 
2-14 Cumulative integral of buoyancy flux and shear production along the domain. . . . . . . . . . . . . . . . 69

2-15 Mixing and Mixing efficiency, or $R i_{b}$, versus jump height. . . . . . . 69

2-16 3D density and velocity comparison for a SFTJ. . . . . . . . . . . 72

2-17 3D density and velocity comparison for a FTJ. . . . . . . . . . 73

2-18 Cumulative integrals of buoyancy flux and shear production along the domain for the 3D run. . . . . . . . . . . . . . . 74

3-1 Theoretical and allowable solution space for WS theory with various

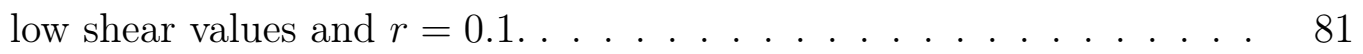

3-2 Dimensional two-layer flow setup. . . . . . . . . . . . . . 83

3-3 Non-dimensional two-layer flow setup with entrainment. . . . . . . . . 83

3-4 Theoretical results with and without entrainment and various shape parameters. ............................ 89

3-5 Shape parameters for sample profiles. . . . . . . . . . . . . . . . . 90

3-6 Behavior of upper layer for jumps with entrainment. . . . . . . . . . . 91

3-7 Initial conditions, development, and fully developed jump for a high shear jump. . . . . . . . . . . . . . . . . . . . 95

3-8 Super- to super- critical transition with high upstream shear . . . . . 97

3-9 Profiles and mode structure of super- to super-critical transition . . . 98

3-10 Super- to sub- critical transition with high upstream shear . . . . . . 100

3-11 Profiles and mode structure of super- to sub-critical transition . . . . 101

3-12 Numerical results compared to theory for two sets of shape parameters. 102

3-13 Solution curves with various combinations of shape parameters. . . . 105

3-14 Numerical results compared to theory for all simulations. . . . . . . . 106

3-15 Wilkinson and Wood (1971) flow structure . . . . . . . . . . . 110

3-16 Jump structures for flows with high upstream shear. . . . . . . . . . . 111

3-17 Regime diagram of jump structures. . . . . . . . . . . . . . . . . . 112

3-18 Vorticity budget of moderate shear jumps. . . . . . . . . . . . . . . . 113

3-19 Vorticity budget of high shear jumps. . . . . . . . . . . . . . . . 114 
3-20 Value of constant $d$ versus shear $s$ with turbulent energy upstream and without turbulent energy upstream. . . . . . . . . . . . . . 117

3-21 Value of constant $d$ versus shear $s$ with turbulent energy downstream. 118

3-22 Simulation and theory results for modified Holland et al. (2002) model with turbulent energy downstream only. . . . . . . . . . . . . . . 119

3-23 $R_{\text {theor }}$ versus $R_{\text {sim }}$ and $q_{\text {efrac,theor }}$ versus $q_{\text {efrac,sim }}$ using modified theory that predicts entrainment . . . . . . . . . . . . . . . 122

3-24 Gradient Richardson number. . . . . . . . . . . . . . . . . . 123

3-25 Flow setup of Wilkinson and Wood (1971) . . . . . . . . . . . . . . 124

3-26 Modified Wilkinson and Wood predictions. . . . . . . . . . . . 127

3-27 Regime diagram of control type. . . . . . . . . . . . . . . . . . . 128

3-28 Example flows with different control locations . . . . . . . . . . . 129

3-29 Effect of varying weir height. . . . . . . . . . . . . . . . . . 131

3-30 Effect of varying distance to weir. . . . . . . . . . . . . . 133

3-31 Effect on entrainment of varying distance to weir. . . . . . . . . . 133

3-32 Mixing efficiency and entrainment for increasing shear. . . . . . . . . 135

3-33 3D simulation results: tracer and velocity fields. . . . . . . . . . . . 137

3-34 3D simulation results: velocity and density profiles. . . . . . . . . . . 139

3-35 3D simulation results: $R i_{g} \ldots \ldots \ldots$. . . . . . . . . . . 140

4-1 Setup of two-layer flow with topography. . . . . . . . . . . . . . . . 147

4-2 Initial density field. . . . . . . . . . . . . . . . . . . . . 152

4-3 Theory with change in depth compared to numerical simulation. . . . 155

4-4 Theory with change in depth compared to numerical simulations. . . 156

4-5 Initial conditions and topography of more realistic flows. . . . . . . . 160

4-6 Higher-mode jump with wedge: flow structure. . . . . . . . . . . 163

4-7 First-mode jump with breaking lee wave: flow structure. . . . . . . . 163

4-8 Higher-mode jump with upstream shear instability: flow strucure. . . 164

4-9 First-mode jump with upstream shear instability: flow structure. . . . 164

4-10 Regime diagram for flows with varying topography: steep topography. 166 
4-11 Regime diagram for flows with varying topography: gentle topographic slope. ............................ 167

4-12 Regime diagram for flows with varying downstream slope: volume flow rate versus topographic slope. . . . . . . . . . . . . . . . . 167

4-13 Water mass budgets development in time. . . . . . . . . . . . . 175

4-14 Water mass budgets development in time. . . . . . . . . . . . . . 176

4-15 Mass source ratios versus volume flow rate. . . . . . . . . . . . . 178

4-16 $R i_{f}$ for jumps with topography. . . . . . . . . . . . . . . . 180

4-17 Mixing in a higher-mode jump with wedge. . . . . . . . . . . . . 181

4-18 Adjusted $R i_{f}$ for jumps with topography. . . . . . . . . . . . . . . . 182

4-19 Mixing in a first-mode jump with wave overturning. . . . . . . . . . . 183

4-20 Scalar variance production $P$ as a function of the flow parameters $s$, $q, h_{T}, T_{T}, \nu$, and the mode of the jump. . . . . . . . . . 185

4-21 Cumulative integral of scalar variance production versus upstream shear for jumps with topography. . . . . . . . . . . . . . 186

4-22 Scalar variance production versus upstream shear . . . . . . . . . . 186

4-23 Regime diagram of $q$ versus $h_{T}$, with color indicating the scalar variance production and the upstream shear. . . . . . . . . . . . . . 187

4-24 Viscosity function. . . . . . . . . . . . . . . . . . . . . 188

4-25 Time-averaged results for a no-slip flow compared to a flow with freeslip: first-mode jump with wave overturning. . . . . . . . . . . . . . . 190

4-26 Richardson number and streamlines for first-mode jump with wave overturning, with and without no-slip condition. . . . . . . . . 191

4-27 Water mass development for flows with and without no-slip condition. 192

4-28 Time-averaged results for a no-slip flow compared to flow with free-slip: higher mode jump with wedge. . . . . . . . . . . . . . . 193

4-29 Time-averaged results for a no-slip flow compared to flow with free-slip: first mode jump with upstream shear . . . . . . . . . . . . . . 195

4-30 Time-averaged results for a no-slip flow compared to flow with freeslip: first-mode jump with wave overturning (inviscid) and first-mode jump with upstream shear instabilities (no-slip). . . . . . . . . . 196 
4-31 Ri $i_{g}$ and streamlines for flow with and without the no-slip condition. . 197 4-32 3D and 2D equivalent simulations of a first-mode jump with wave overturning. . . . . . . . . . . . . . . . . . 199

4-33 3D and 2D equivalent simulations of a higher-mode jump with a wedge. 201

4-34 Observations of Stellwagen Bank from Pineda (personal communication).203

4-35 Density profiles calculated from observed temperature and salinity profiles. . . . . . . . . . . . . . . . . . . 203

4-36 Stellwagen Bank topography. . . . . . . . . . . . . . . . . . . 204

4-37 Stellwagen Bank simulation compared to observations. . . . . . . . 206 


\section{List of Tables}

1.1 General Properties of observed hydraulic jumps . . . . . . . . . . . 29

1.2 Non-dimensional parameters of observed hydraulic jumps . . . . . . . 30

3.1 Shape functions for a set of simulations including super- to super- and super- to sub-critical jumps. . . . . . . . . . . . . . . . 103

3.2 Idealized density and velocity profiles and corresponding mode- 1 phase speeds. . . . . . . . . . . . . . . . . . . . . . 108

4.1 Non-dimensional parameters of observed hydraulic jumps. . . . . . . 161 


\section{Chapter 1}

\section{Introduction}

Hydraulically controlled flow is ubiquitous in the world's ocean, seas, and channels. This includes hydraulic jumps, which occur in a variety of situations. River outflows can excite undular bores in the stratified ambient fluid into which they propagate (Nash and Moum, 2005). Other examples include atmospheric undular bores, such as the Morning Glory in Australia and Oklahoma, sea breeze fronts, and thunderstorm outflows (Rottman and Simpson, 1989), as well as downslope wind storms in the lee of mountains, such as those observed by Lilly (1978) in Colorado, and by Doyle et al. (2005) in Greenland. Internal hydraulic control and jumps can occur in the abyssal ocean as dense water flows over sills between deep basins (Polzin et al., 1996; St.Laurent and Thurnherr, 2007; Alford et al., 2013). They also occur in connecting channels such as Gibraltar, where the amount of mixing affects the Mediterranean water properties, and in peripheral channels such as Knight Inlet and Hood Canal (Sánchez-Garrido et al., 2011; Klymak and Gregg, 2003; Farmer and Armi, 1999; Gregg and Pratt, 2010).

Although many flows exhibit hydraulic control and a hydraulic jump, the properties of the flow and the jumps can vary significantly. For example, the flow in Knight

Inlet is tidally driven and therefore transient, whereas the flows in Gibraltar and the Pre-Bosphorus are exchange flows (Sánchez-Garrido et al., 2011; Gregg and Özsoy, 2002) with a steady component. In Hood Canal and Gibraltar, the channel width is large enough for rotation to affect the flow (Gregg and Pratt, 2010; Gregg and Özsoy, 
2002). Details of the flow such as the density stratification and the shape of the topography also affect the form of the hydraulic jump. All of these factors combine to form a unique jump in each location. However, most can be categorized based on their general properties, and understanding what types of jumps can occur under what conditions is the goal of this thesis.

Improving our understanding of hydraulic jumps is important mainly due to the mixing that they cause. Intense mixing can occur in a hydraulic jump, and this affects the water properties such as temperature, salinity, and oxygen distribution. A significant amount of energy can also be dissipated in a hydraulic jump (Klymak and Gregg, 2004). Understanding hydraulic jumps and the mixing that they cause affects the understanding of ocean circulation because mixing determines the properties of water such as Mediterranean outflow water through the Strait of Gibraltar. The mixing that occurs in hydraulic jumps also affects biological productivity by altering the distribution of dissolved oxygen and nutrients in the water column. Furthermore, the local energy budget is affected due to the dissipation of energy that can occur in a hydraulic jump. In atmospheric flows, understanding the jump properties could affect flight planning due to turbulence, and weather prediction based on the winds generated by the flowfield. For all of these reasons, a better understanding of internal hydraulic jumps is desired.

\subsection{Hydraulic Jumps in the Environment}

Hydraulic jumps in several locations have been studied in detail. For example, Knight Inlet, a peripheral channel located in southwest British Columbia, Canada, has been studied observationally by Farmer and Armi (1999) and Klymak and Gregg (2004), and numerically by Cummins (2000), Lamb (2004), and Afanasyev and Peltier (2001), among others. The location and bottom topography are shown in figure 1-1.

Knight Inlet experiences a tidally driven flow over a sill, and hydraulic jumps occur 

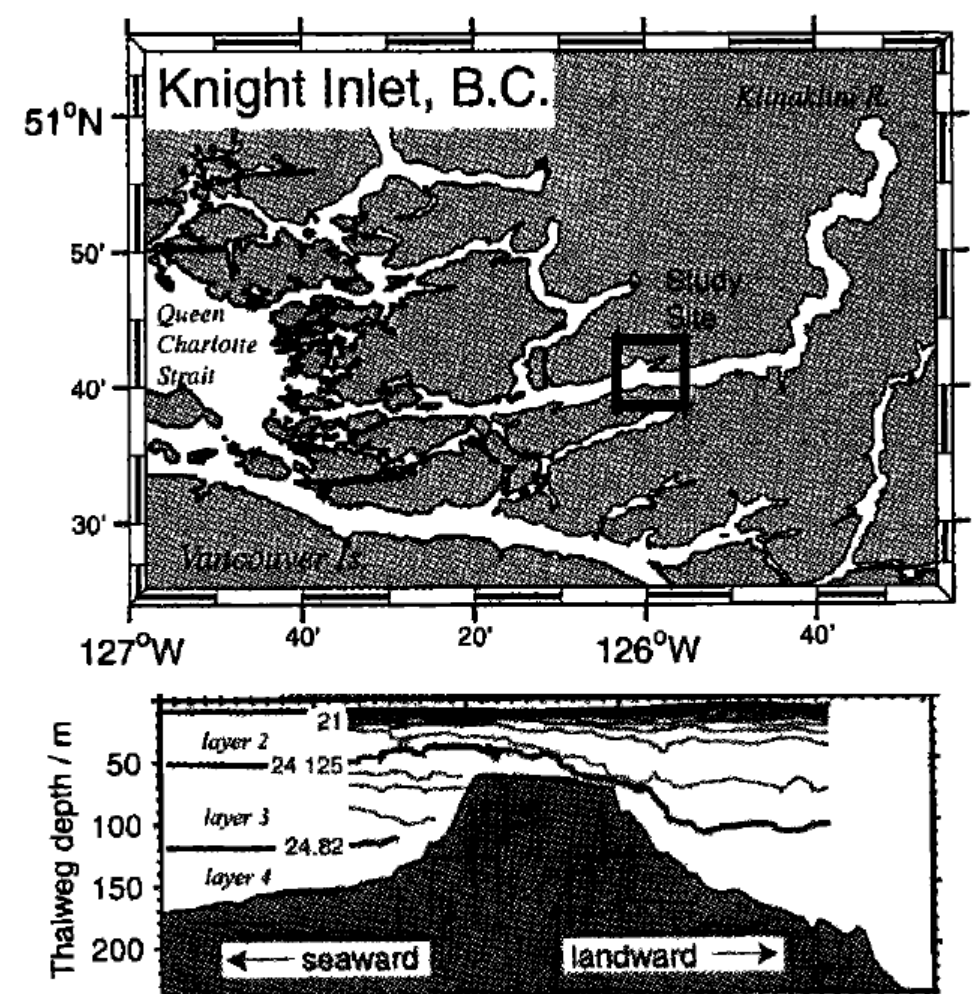

Figure 1-1: Knight Inlet location and topography (reprinted from Klymak, J. and Gregg, M. (2001). Three-dimensional nature of flow near a sill. Journal of Geophysical Research, 106(C10):22295 - 22311. (C)2001 American Geophysical Union. Used with permission.) 
on alternating sides, in the lee of the sill. In the summer, the density profile in Knight Inlet has a shallow mixed layer at the surface, with density increasing significantly within the upper twenty meters, and then increasing slowly through the remaining depth. In the winter, the density has a constant stratification through the upper forty meters, and a constant but smaller stratification through the remaining depth (Farmer and Smith, 1980). There are also differences in stratification and topography between the two sides of the sill, which could produce qualitatively different jumps depending on the season and the phase of the tide.

Observations of the flow in Knight Inlet are shown in figure 1-2 (Klymak and Gregg, 2004). The figure shows a hydraulic jump downstream of the sill, where at about $x=1000 \mathrm{~m}$, the lower isopycnals plunge down over the sill, and then, around $x=1500 \mathrm{~m}$, they jump back up toward the surface in a hydraulic jump. Knight Inlet also exhibits another interesting structural feature called a wedge. The wedge is a region of well-mixed, stagnant fluid of intermediate density just ahead of the jump. The wedge is visible in figure 1-2a centered at around $x=1200 \mathrm{~m}$ and a depth of 20 m. The hydraulic jump in Knight Inlet is an important factor in the energy budget, dissipating about one-third of the barotropic energy in the inlet (Klymak and Gregg, 2004), which is one reason an understanding of the details of the hydraulic jump is important.

Due to the extensive studies, the details of the hydraulic jump that occurs in Knight Inlet are fairly well understood. However, there is still some debate about the details of the dynamics. For example, the mechanism that causes mixing ahead of the jump and in the wedge region is still unclear. The flow in Hood Canal, however, is not as well understood. Hood Canal is another tidally driven peripheral channel, located in Washington State, off Puget Sound, as seen in figure 1-3a. A recent paper by Gregg and Pratt (2010) suggests that a hydraulic jump may occur over a sill in Hood Canal, although whether there is a jump and the properties of the jump are uncertain. Several important properties of Hood Canal are different from Knight Inlet. The density profile is one example. The density field through the depth and along the channel is 


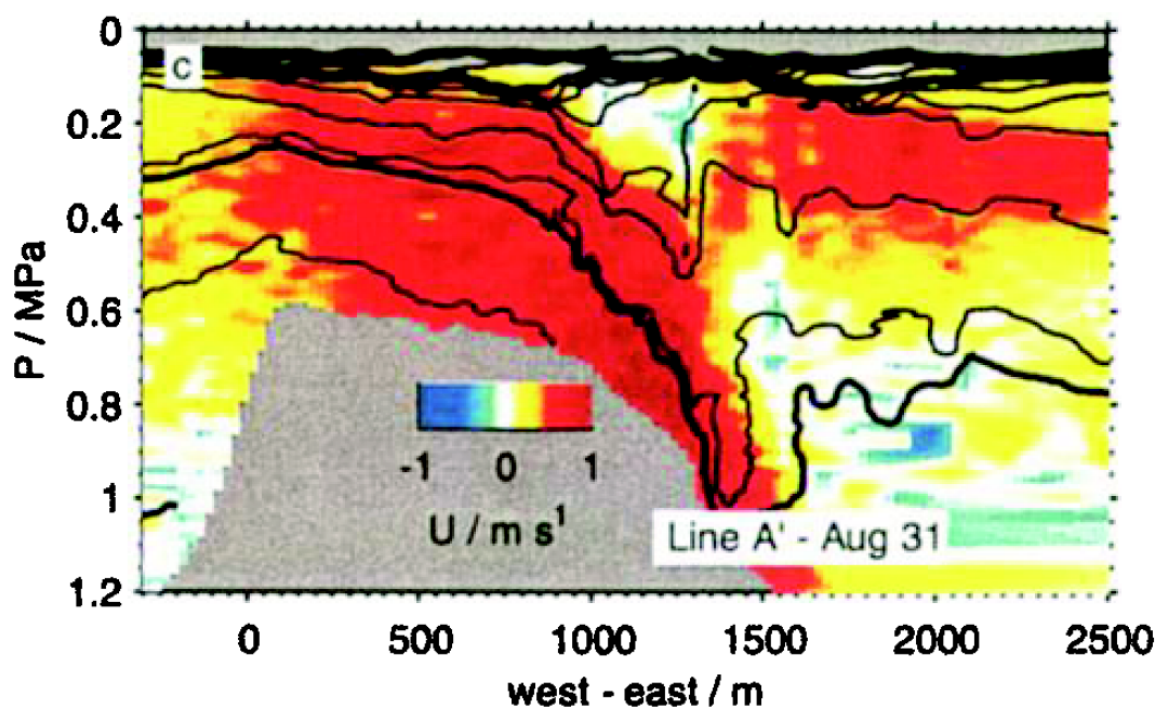

Figure 1-2: Hydraulic jump in Knight Inlet showing along-channel velocity magnitude in color and density contours in solid lines (reprinted from Klymak, J. and Gregg, M. (2001). Three-dimensional nature of flow near a sill. Journal of Geophysical Research, 106(C10):22295 - 22311. (C)2001 American Geophysical Union. Used with permission.)

shown in figure 1-3b. The deeper isopycnals again plunge over the sill at an alongchannel distance of 20 to 21, jumping back up around 23, which is the feature that may be a hydraulic jump. However, the wave speeds do not transition from superto sub-critical across the jump as expected (Gregg and Pratt, 2010). Hood Canal has recently experienced fish kills, motivating an interest in a better understanding of the flow there (Josberger and Cheng, 2005). The mixing in internal hydraulic jumps affects the distrubution of dissolved oxygen and nutrients, which could influence the fish populations in Hood Canal, and may affect the magnitude of these fish kill events.

The Strait of Gibraltar and the Pre-Bosphorus Channel are both exchange flows that exhibit hydraulic jumps (Wesson and Gregg, 1994; Gregg and Özsoy, 2002). The flow over Camarinal Sill in the Strait of Gibraltar is shown in figure 1-4, where the lower layer flows from right to left. The figure shows accoustic backscatter, and the interface can be identified where the scatter is larger. The jump occurs at $x \approx 2400$ m. As with Hood Canal, the Strait of Gibraltar is also wide enough to be affected by rotation, complicating the understanding of the flow. The mixing that occurs in Gibraltar dissipates barotropic tidal energy and affects local water properties, such 

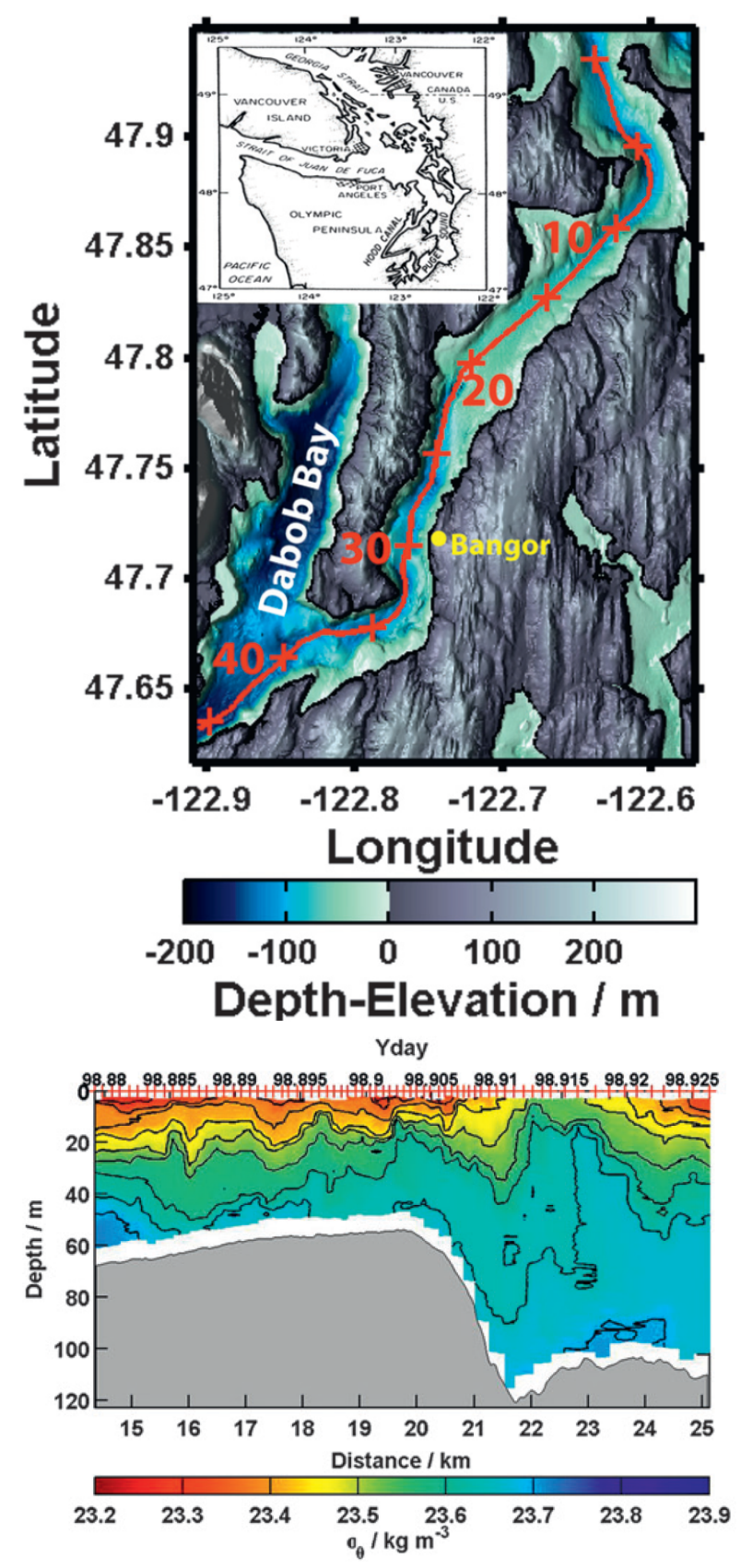

Figure 1-3: (a) Location of Hood Canal and (b) along-channel cross-section of the potential density field (reprinted from Gregg, M. and Pratt, L. (2010). Flow and hydraulics near the sill of Hood Canal, a strongly sheared, continuously stratified fjord. Journal of Physical Oceanography, 40:1087 - 1105. Figures 1 and 13. (C)2010 American Meteorological Society. Used with permission.) 
as salinity and temperature (Sánchez-Garrido et al., 2011), which motivates an understanding of the hydraulic jump in the Strait of Gibraltar.

The flow in the Pre-Bosphorus Channel is shown in figure 1-5, where the lower layer moves from left to right. This flow exhibits a homogeneous region of intermediate density near the jump, which may be considered a wedge, located at $37 \mathrm{~km}$ and a pressure of $p=0.5 \mathrm{MPa}$. The feature is a region of intermediate density between the upper and lower layers, just ahead of the jump. The Pre-Bosphorus also experiences low oxygen levels deep in the water column, which affects the biologoical productivity. This is particularly important in such a highly populated region, where the economy may also be affected through the fishing industry if biological productivity is reduced (Ünlüata et al., 1993). In both the Strait of Gibraltar and the Pre-Bosphorus Channel, the density stratification is more two layered than either Hood Canal or Knight Inlet. This is because both are exchange flows, so the upper and lower layer waters have different sources. Therefore, the simple two-layer approximation used in most of this work may be more applicable for these locations.

The tide forces a flow back and forth over Stellwagen Bank, which results in a hydraulic jump forming in the lee of the sill (Scotti et al., 2007). The location and topography of Stellwagen Bank are shown in figure 1-6. This type of flow differs from the previous examples because it is not contained within a narrow channel. Scotti et al. (2007) studied the flow in Stellwagen Bank in the context of non-linear internal waves, although the flow spilling over the sill could also be considered in the context of a hydraulic jump by applying the ideas investigated in this work, as hydraulic jumps uppear to form in their numerical model results.

A summary of some of the important properties of the hydraulic jumps described above is given in table 1.1. Non-dimensional parameters indicating the importance of friction, rotation, and time-dependence are give in table 1.2. The importance of friction is quantified by $\frac{C_{D} L}{D}$, which compares the drag coefficient $C_{D}$, the characteristic along channel length $L$, and the depth $D$ of the channel. If the drag coefficient is 


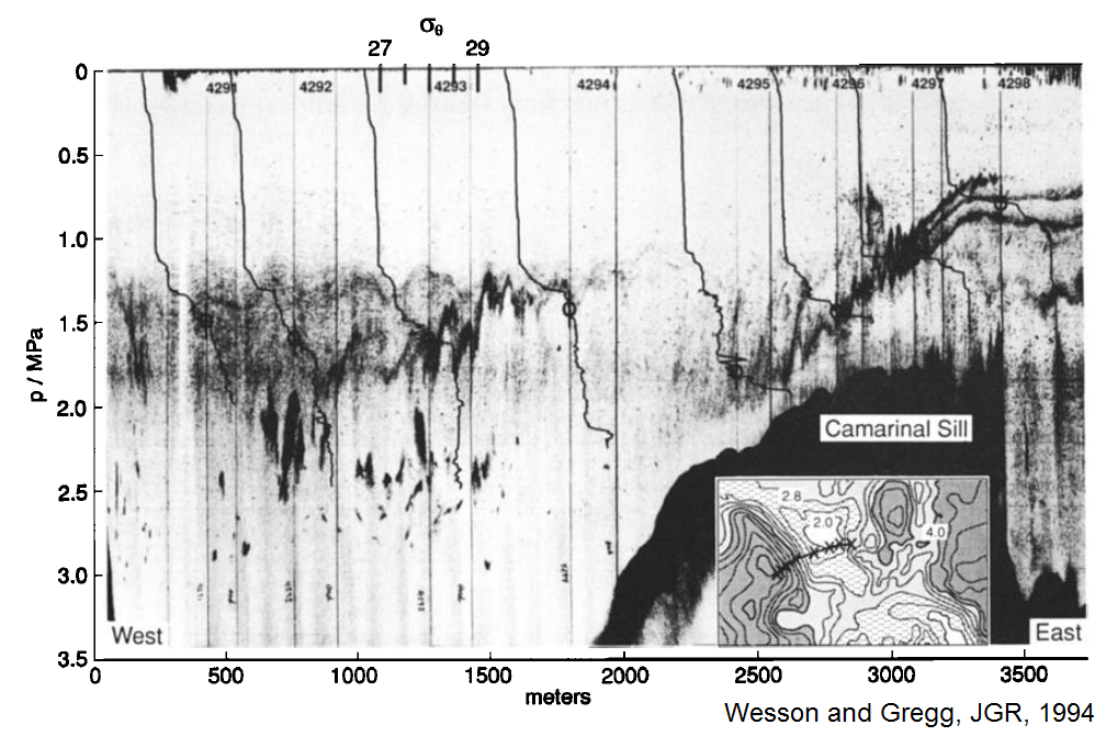

Figure 1-4: Acoustic backscatter showing a hydraulic jump in the Strait of Gibraltar at Camarinal Sill (reprinted from Wesson, J. and Gregg, M. (1994). Mixing at Camarinal sill in the Strait of Gibraltar. Journal of Geophysical Research. (C1994 American Geophysical Union. Used with permission.)

large or acts over a sufficiently large distance compared to the depth of the channel, $\frac{C_{d} L}{D} \gtrsim O(1)$ and friction is important. Time-dependence is quantified by the length $(L)$ and time $\left(T_{u}\right)$ scales of the flow and the fastest internal wave speed $\left(c_{-}\right.$of the flow. If disturbances are unable to propagate away from the region of the jump before the forcing changes significantly, $\frac{L}{c_{-} T_{u}} \gtrsim O(1)$ and the temporal variation of the forcing is important. The importance of rotation depends on the internal wave speed $c$, the width of the channel $W$, and the Coriolis frequency $f$. If the time a wave takes to traverse the width of the channel is comparable to the timescale of Earth's rotation, then the rotation impacts the dynamics of the flow. The values indicate that time-dependence is important in Knight Inlet and Hood Canal, which are tidally driven, but not in the Pre-Bosphorus, which is an exchange flow. In most locations, friction may be important, but it is not obviously a significant factor. Rotation is most important in the Strait of Gibraltar, Hood Canal, and Stellwagen Bank, and not very important in Knight Inlet and the Pre-Bosphorus. The range of properties suggests that hydraulic jumps may take on a variety of structural features, as they can be influenced by a combination of these factors. The non-dimensional parameter 

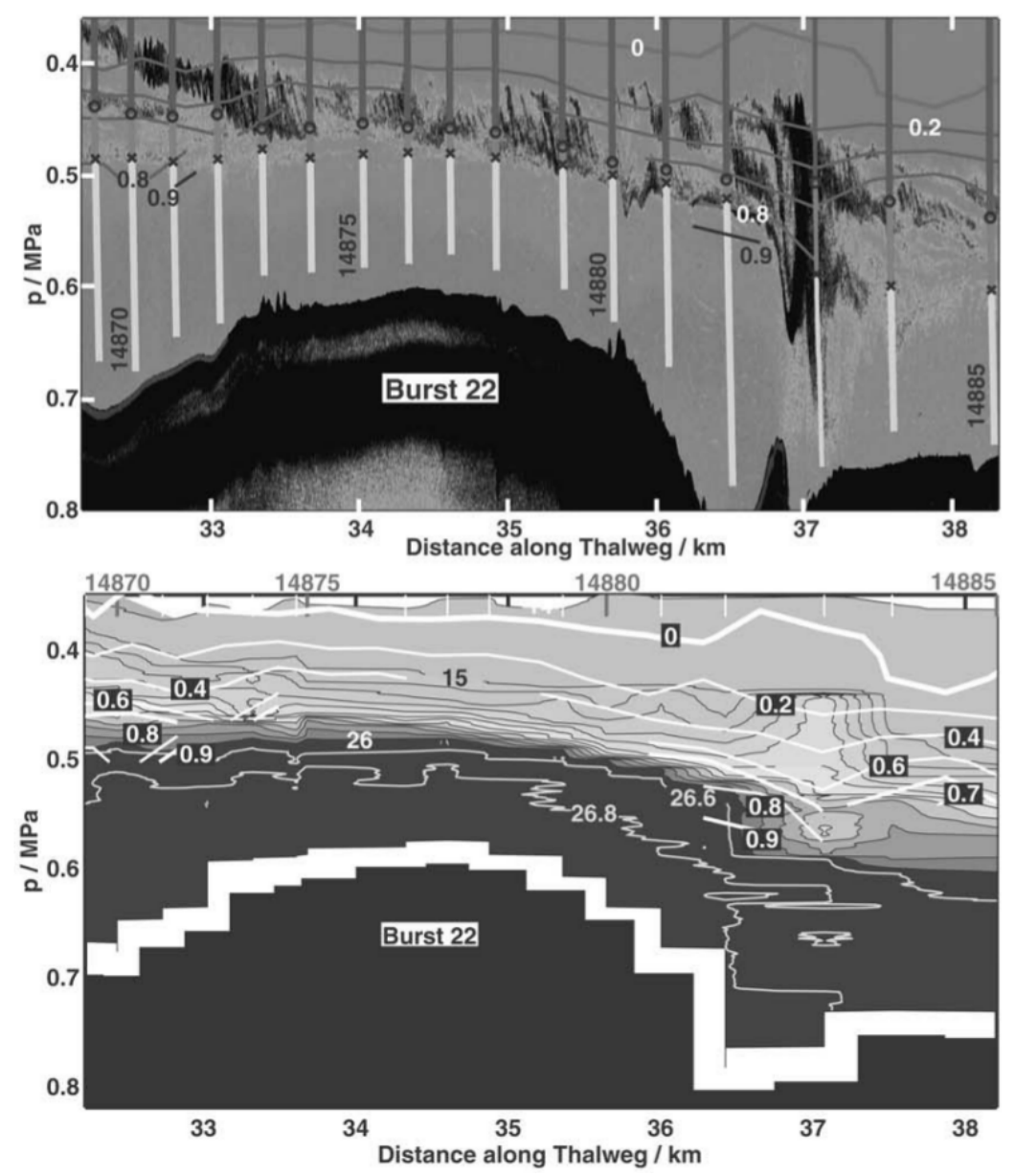

Figure 1-5: Acoustic backscatter (top) and density and velocity contours (bottom) showing a hydraulic jump in the Pre-Bosphorus Channel (reprinted from Gregg, M. and Özsoy, E. (2002). Flow, water mass changes, and hydraulics in the Bosphorus. J. of Geophysical Research, 107(C3). (C)2002 American Geophysical Union. Used with permission.) 

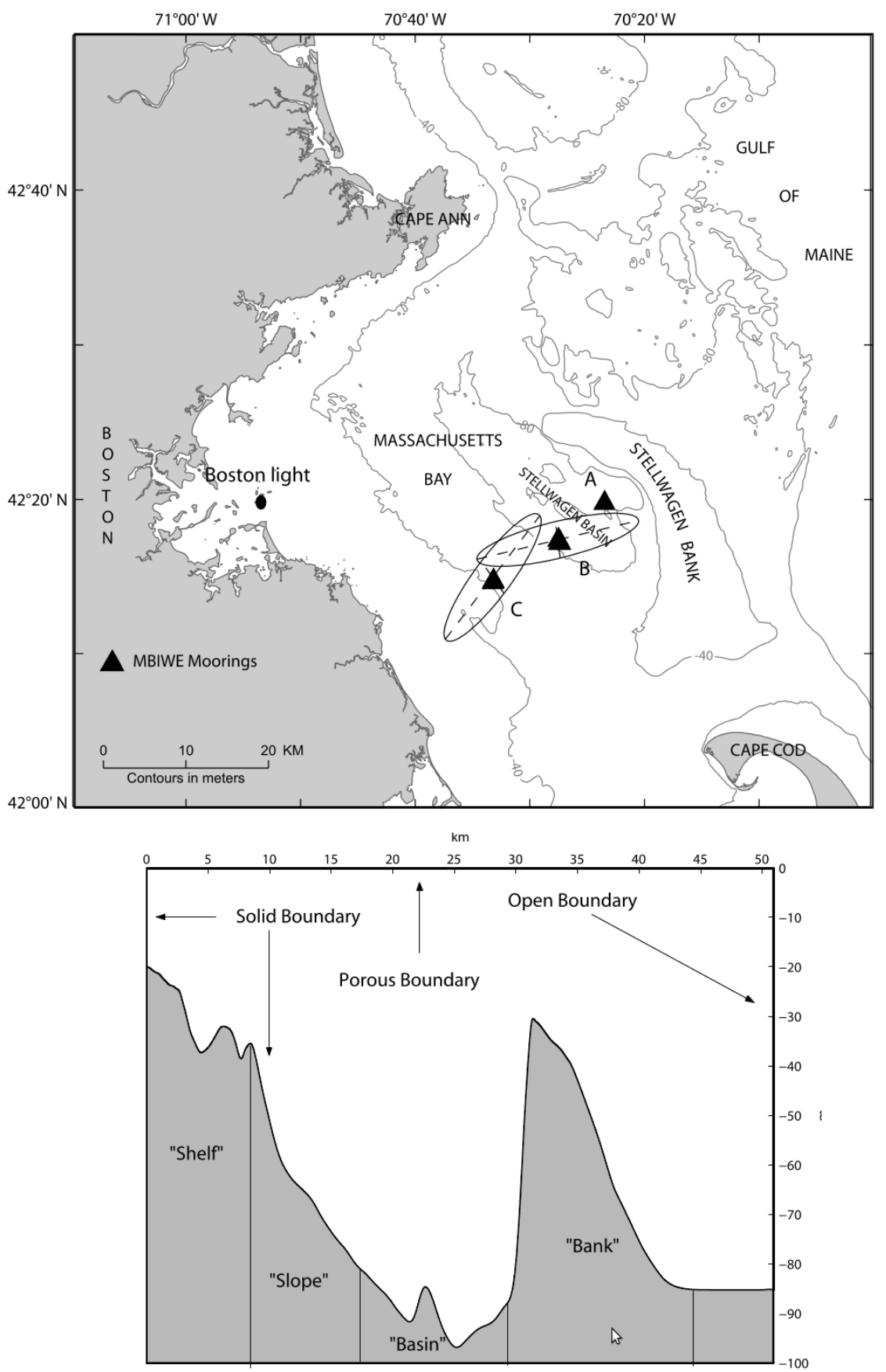

Figure 1-6: Stellwagen Bank location and topogarphy (reprinted from Scotti, A., Beardsley, R., and Butman, B. (2007). Generation and propagation of nonlinear internal waves in Massachusetts Bay. Journal of Geophysical Research, 112. (C)2007 American Geophysical Union. Used with permission.) 
describing the importance of friction is also included for the Faroe Bank Channel and the Denmark Strait, which are dense overflows. In these locations, the topographic slope is much more gentle, and friction is therefore more important (Pratt and Whitehead, 2007). Friction can prevent a jump from occuring, and gentle topography allows entrainment to modify the upstream flow before a hydraulic jump can occur, changing the properties of or eliminating a jump. Furthermore, the width of these channels allows rotation to influence the flow (Pratt et al., 2007), which would modify the properties of a jump, if one occurs. For these reasons, the results found in this work are more applicable to the abrupt jumps in the coastal ocean and peripheral channels.

The flows studied in this work are most applicable to Knight Inlet, Hood Canal, and Stellwagen Bank, where the upstream flow is unidirectional through the depth of the water column, rather than an exchange flow. However, the effect of upstream shear is important because even these tidally driven flows develop shear as the stratified water is forced over topography and develops a vertical velocity shear. The effect of this upstream shear on internal hydraulic jumps the main focus of this work.

\begin{tabular}{lllllll}
\hline Location & Depth & Time Var. & Strat. & Rotation & Slope & Exchange \\
\hline Hood Canal & Shallow & Time-dep. & Cts & Rotation & 0.03 & No \\
Knight Inlet & Shallow & Time-dep. & Cts & No rotation & $0.05-0.2$ & No \\
Gibraltar & Shallow & Time-dep. & 2-layer & Rotation & 0.05 & Yes \\
Pre-Bosphorus & Shallow & Steady & 2-layer & No rotation & 0.01 & Yes \\
Stellwagen Bank & Shallow & Steady & Cts & Rotation & $0.003-0.04$ & No \\
\hline
\end{tabular}

Table 1.1: General Properties of observed hydraulic jumps. Information from Klymak and Gregg (2003); Lamb (2004); Gregg and Pratt (2010); Sánchez-Garrido et al. (2011); Gregg and Özsoy (1999); Scotti et al. (2007).

Hydraulic control, possibly including internal hydraulic jumps, has also recently been indentified as a possibly important factor in the intense mixing of deep channels such as the Romanche fracture zone, Vema Passage, and smaller passages in the Mid-Atlantic Ridge (St.Laurent and Thurnherr, 2007; Polzin et al., 1996). Although this thesis is guided by the geometry and parameters of shallow tidally driven 
flows over topography, these deep channels may be a useful application of the findings.

\begin{tabular}{lccc}
\hline & Friction & Time-Dependence & Rotation \\
Important if: & $\frac{C_{d} L}{D} \gtrsim O(1)$ & $\frac{L}{c_{-} T_{u}} \gtrsim O(1)$ & $\frac{R_{d, i}}{W}=\frac{c}{W f} \lesssim O(1)$ \\
\hline Hood Canal & 0.05 & 1.2 & 0.9 \\
Knight Inlet & 0.08 & 0.7 & 1.3 \\
Gibraltar & 0.05 & 1.7 & 0.5 \\
Pre-Bosphorus & 1 & 0.8 & 8 \\
Stellwagen Bank & 0.2 & 1.4 & 0.009 \\
Denmark Strait & 1 & - & - \\
Faroe Bank Channel & 2 & - & - \\
\hline
\end{tabular}

Table 1.2: Non-dimensional parameters of observed hydraulic jumps. Parameters estimated from data in Klymak and Gregg (2003); Lamb (2004); Gregg and Pratt (2010); SánchezGarrido et al. (2011); Gregg and Özsoy (1999); Scotti et al. (2007); Pratt and Whitehead (2007).

\subsection{Previous Findings and Open Questions}

Idealized hydraulic jumps have been studied previously, for example by Wood and Simpson (1984) and Klemp et al. (1997), and more recently by Borden and Meiburg (2013a). These authors considered two-layered hydraulic jumps without upstream shear, and each studies a different theoretical approach to dealing with the change in surface pressure across the jump. In hydraulic jumps in the environment, the height of the surface of the water changes across the jump. However, when modeling these jumps, a rigid-lid assumption is often made. The change in surface height is then accounted for through a change in surface pressure across the jump, which can be approximated in different ways. Wood and Simpson (1984) assume that energy is conserved in the upper layer, while Klemp et al. (1997) assume that energy is conserved in the lower layer. Klemp et al. (1997) note that their approach is most applicable for flows with small upstream shear and a thin upstream lower layer, and the Wood and Simpson (1984) approach is more applicable for jumps with larger upstream shear. This is also consistent with the high shear jumps studied in chapter 3, in which fluid is entrained and mixed into the lower layer; this mixing would dissipate energy in 
the lower, contradicting the assumptions of the Klemp et al. (1997) model. Borden and Meiburg (2013a) conserve vorticity, which eliminates the pressure from the governing equations, avoiding this type of assumption. Their model balances baroclinic production of vorticity with vorticity divergence, which is consistent with low shear simulations, and their model predictions fall between those of Wood and Simpson (1984) and Klemp et al. (1997).

All of these two-layered models fail to predict hydraulic jumps as the upstream shear increases, and provide no guidance as to how the physics of the flow changes with increasing shear. Simulations show the dominant vorticity balance is between the mean and turbulent vorticity divergence terms for flows with large upstream shear, suggesting that the vorticity conservation model developed by Borden and Meiburg (2013a) is no longer applicable. Wilkinson and Wood (1971) studied the one-and-ahalf-layer problem theoretically and experimentally, which allows upstream shear that is determined by the upstream lower layer velocity, due to the stagnant upper layer. Their work suggests that entrainment and continuous velocity and density profiles may be important. A comprehensive understanding of the two-layer problem with an active upper layer and independent upstream shear has not yet been described, and addressing this idealized problem is one of the goals of this thesis. The results show that the two-layer approximation is limited as shear increases because the continuous velocity profile becomes important, with a flow reversal near the interface developing in some cases.

Upstream shear commonly occurs in hydraulic jumps in the environment due to the flow over topography in locations such as Knight Inlet and Hood Canal, and as a natural part of an exchange flow in locations such as the Pre-Bosphorus and the Strait of Gibraltar. Therefore, a better understanding of how jump properties are affected by upstream shear is important.

Existing hydraulic jumps in the environment have also been studied previously. Knight Inlet, for example, has been extensively studied numerically (Klymak and 
Gregg, 2003; Stashchuk and Vlasenko, 2007; Afanasyev and Peltier, 2001; Lamb, 2004). Klymak and Gregg (2003) conducted simulations of tidally varying flow over a sill topographically similar to Knight Inlet, using a 2D hydrostatic model. They studied the effect of asymmetric sill topography and density stratification in the along channel direction. They conclude that the density asymmetry has an important role in the asymmetry of the flow observed in Knight Inlet, including the flow separation.

Stashchuk and Vlasenko (2007) used a non-hydrostatic, Boussinesq, 2D model to conduct time-dependent simulations with smoothed topography based on the sill in Knight Inlet and a density profile based on observations. They focused on whether shear instabilities were necessary to forming the hydraulic jump in the lee of the sill, and concluded that the shear instabilities and solitary waves are formed separately. Afanasyev and Peltier (2001) also performed idealized simulations of Knight Inlet using a 2D, Boussinesq, non-hydrostatic model. They concluded that the mixing downstream of the sill is caused by breaking lee waves rather than upstream shear instability, and that formation of the wedge region is necessary for the flow to become hydraulically controlled. Lamb (2004) also studied Knight Inlet using a 2D, non-hydrostatic model with vertical eddy diffusivity localized near the bottom, and a no-slip bottom boundary condition, allowing him to investigate the conditions under which flow separation occurred. He concluded that flow separation was an important factor influencing the flow in Knight Inlet, but that breaking lee waves caused the formation of the jump. Conversely, Armi and Farmer (2002) claim small-scale instabilities cause the mixing that generates fluid of intermediate density in the wedge region in Knight Inlet. In general, Winters and Armi (2013) conclude that shear instability is responsible for the energy dissipation in flows with low frequency of forcing and/or low topographic Froude number. These conditions occur for a flow that is quasi-steady, allowing a hydraulic jump to develop in the lee of the sill, and has sufficiently high topography to cause a jet to form over the sill and the flow to be controlled. This suggests that the mechanism responsible for mixing depends on details of the flow and the location of the jump. However, many of these studies are specific to Knight Inlet, whereas jumps in other channels may have different proper- 
ties determined by different physical processes.

Other open questions about properties of hydraulic jumps include under what conditions a wedge region forms, and from where the water in the wedge originates. A wedge is found in Knight Inlet, as noted in Klymak and Gregg (2004), and shown in figure 1-2. A wedge region at $37 \mathrm{~km}$ along the thalweg is also visible between the sill and hydraulic jump in the Pre-Bosphorus Channel (Gregg and Özsoy, 2002), shown in figure 1-5. Klymak and Gregg (2004) suggest that the wedge may depend on the tidal cycle. However, the Pre-Bosphorus is almost steady, and a wedge-like region also occurs in other steady hydraulic jumps, such as in atmospheric flows. For example, Peltier and Clark (1979) did numerical simulations of lee waves motivated by the atmospheric application of steady flows over mountains, and found a homogeneous region of stagnant fluid. Smith (1985) calculated theoretical properties of the the wedge in a steady, infinitely deep flow with constant stratification. More recently, Doyle et al. (2005) published observations of mountain lee waves over Greenland that show a hydraulic jump and a region of constant potential temperature that resembles a wedge. However, a wedge region does not appear in Hood Canal (Gregg and Pratt, 2010). Afanasyev and Peltier (2001) claim that a wedge region will occur if the flow is hydraulically controlled, although the observations of Gregg and Pratt (2010) indicate that the flow in Hood Canal is supercritical, and a hydraulic jumps appears to occur. Although the wedge seems to be a common feature of hydraulic jumps, the conditions under which it exists and its effect on the jump structure and mixing are unknown. Furthermore, Farmer and Armi (1999) say that the wedge causes the two layers to decouple. If the two layers are decoupled, the two-layer theories discussed in chapters 2 and 3 may be innapplicable. The cause and effects of the wedge region on the jump structure and mixing are therefore important.

Open questions about naturally occuring internal hydraulic jumps include under what conditions a wedge region develops and where the fluid within the wedge originates, how stratification and topography affect the jump properties, and when shear instability versus wave overturning drives the development of the jump. Even in the 
well studied Knight Inlet, questions remain about the physical processes that determine jumps properties. Furthermore, most hydraulic jumps in the environment are not as well studied as Knight Inlet, and predictions about the properties of these jumps could be made based on a more complete understanding of idealized hydraulic jumps. These questions motivate this work on internal hydraulic jumps, although highly idealized jumps are considered first. Chapter 4 begins to address some of these questions, which are more relevant to hydraulic jumps in the environment.

\subsection{Outline of Thesis}

This work focuses on idealized flows in chapters 2 and 3, with some more realistic simulations described in chapter 4 to draw connections between observed hydraulic jumps and the idealized work. Shallow, steady, two-layer flows without rotation are considered in chapters 2 and 3. Although these basic flows are much simpler than those seen in the environment, studying such idealized flows illustrates the types of jumps that might be observed in nature, and allows some of the factors that determine the jump properties to be identified. In chapter 2, the simplest approximation of an internal hydraulic jump is considered. A two-layer flow with a flat bottom and rigid lid is studied by comparing existing two-layer theories, which are extended to include upstream shear. Upstream shear is an important factor in all of the jumps described in $\S 1.1$, which includes jumps that develop shear due to flow over topography, and jumps that occur in exchange flows with a natural background shear. The theoretical solution space of the theories is identified, and used to guide numerical simulations that include continuous velocity and density profiles. The simulations illustrate the jump structures that can develop and the mixing that occurs within the jump.

The two-layer theories considered in chapter 2 fail to provide jump solutions as the upstream shear between layers increases. How this modifies the jump is studied in

chapter 3. One of the two-layered theories is modified to allow entrainment and shape functions, which account for the continuous velocity profiles. Adding entrainment or 
shape functions allows the theory to produce solutions for higher shear jumps. Predicting the entrainment using the upstream shear and including shape parameters for the velocity profiles highlights the important physics of the flow. However, the theory is very sensitive to the value of the shape functions, and therefore cannot be used to predict the jump height and entrainment, given the upstream conditions. This indicates that the two-layer approximation is limited, particulaly for high shear jumps.

To draw connections between the highly idealized work of chapters 2 and 3 and the observed hydraulic jumps described above, time-dependence, variations in topography, and more continuous density stratifications are considered in chapter 4. How these properties modify the structures of internal hydraulic jumps is investigated. First, the basic two-layered theory with entrainment is modified to include a change in depth across the jump. Although the two-layer approximation had limited applicability in chapter 3 , realistic jumps tend to have lower shear, so its use is again investigated here. How the theory changes when a change in depth is added is generally consistent with how the simulation results differ from the basic theory. However, flows with continuous velocity and density profiles are much more complex than the two-layer approximation can accomodate, so again the theory cannot be used to accurately predict jump properties. Numerical simulations including topography, various density stratifications, and some time-dependence are therefore used to qualitatively categorize the jumps and investigate questions related to mixing and jump structure. 


\section{Chapter 2}

\section{Internal Hydraulic Jumps with Low Upstream Shear}

Reprinted Ogden and Helfrich (2016) with permission of Cambridge University Press.

\subsection{Introduction}

The focus of this chapter is non-rotating internal hydraulic jumps in two-layer flows with upsteam shear. These are relevant in narrow channels with sills, such as Knight Inlet and the Pre-Bosphorus Channel (Klymak and Gregg, 2004; Gregg and Özsoy, 2002). The flow in these channels can often be approximated as a two-layer flow with a chosen isopycnal as the interface. Particularly in cases such as the Pre-Bosphorus Channel, two distinct layers are clearly evident in the exchange flow due to the salin-

ity and temperature differences of the source waters (Gregg and Özsoy, 2002). The opposing flows also suggest that the effects of ambient vertical shear upstream of a jump could be significant. In flows such as Knight Inlet, a division into two distinct layers is less obvious, although the flow over topography tends to form a fast moving jet that is distinct from the slower upper layer, resulting in a flow with upstream shear. A two-layer approximation provides a convenient simplification for analyzing these flows. 
Most existing theories treat the flow as two-layered, ignore the mixing and internal dynamics of the jump, and use shock-joining theories to match the upstream and downstream conditions (Wood and Simpson, 1984; Klemp et al., 1997; Li and Cummins, 1998). The two-layer problem is typically approached by conserving mass and momentum flux through the jump. However, because of the rigid-lid imposed at the surface, an additional assumption is then required to close the problem due to the change in surface pressure across the jump. This assumption determines how mechanical energy dissipation within the jump is distributed between the layers. Two-layer model closures to this problem include those by Wood and Simpson (1984), which conserves energy in the contracting layer, and Klemp et al. (1997), which conserves energy in the expanding layer. Li and Cummins (1998) proposed a model in which the energy loss can be partitioned arbitrarily between the two layers. Recently, Borden and Meiburg (2013b) proposed an approach based on vorticity conservation across the jump. By integrating the vorticity equation through the jump, they balance the baroclinic production of vorticity in the jump with the downstream flux of vorticity on the vortex sheet between the fluid layers. The appealing aspect of their model is that the apparently arbitrary choice of how to distribute dissipation is replaced with a constraint on the vorticity. This results in dissipation in both layers such that the Bernoulli head difference between the layers is conserved across the jump (Borden and Meiburg, 2013a; White and Helfrich, 2014). However, in a dissipative turbulent flow, spatial variations in dissipation can lead to production of vorticity within the jump so that the divergence in vorticity advection is not necessarily balanced exactly by baroclinic production (White and Helfrich, 2014). Borden and Meiburg (2013b) also extended this idea to allow a finite thickness shear layer downstream of the jump, and the theory agrees reasonably well with numerical model simulations for flows without upstream shear.

The two-layer studies discussed above have focused primarily on internal bores advancing into a resting two-layer stratification. In many oceanographic situations upstream shear is present and likely important. Examples include jumps in dense overflows (Alford et al., 2013) and exchange flows, such as the Strait of Gibraltar and 
the Pre-Bosphorus channel (Sánchez-Garrido et al., 2011; Klymak and Gregg, 2004; Gregg and Özsoy, 2002), that have upstream shear by definition. However, the effect of upstream shear has not been fully investigated in the layer models. Klemp et al. (1997) suggested that with sufficient upstream shear, their closure of energy conservation in the expanding layer might fail, and that the Wood and Simpson (1984) closure might be more appropriate.

In an effort to address the issue we explore the behavior of two-layer jumps with upstream shear using the energy closures considered by Wood and Simpson (1984), Klemp et al. (1997), and the Borden and Meiburg (2013b) vorticity conservation model. We also extend the work of Borden et al. (2012), which includes a downstream shear layer and resulting inter-layer energy exchange, to include upstream shear. The physically allowable solution space of the two-layer theories is carefully identified by consideration of the stability of the flow to long interfacial waves and the requirement that energy must be lost across the jump.

These two-layer theories neglect non-hydrostatic processes, turbulence, and mixing within the hydraulic transition between the upstream and downstream states. These models can be modified to parameterize the effects of mixing (c.f. Borden and Meiburg, 2013b). However, these modifications do not directly incorporate the detailed dynamics within the jumps and need to be tested. Since understanding the role of mixing is one of the primary motivations for studying internal jumps, numerical simulations of internal hydraulic jumps are performed with a non-hydrostatic numerical model that allows continuous density and velocity profiles and turbulent mixing. The numerical results are then compared with the predictions from the two-layer theories. 


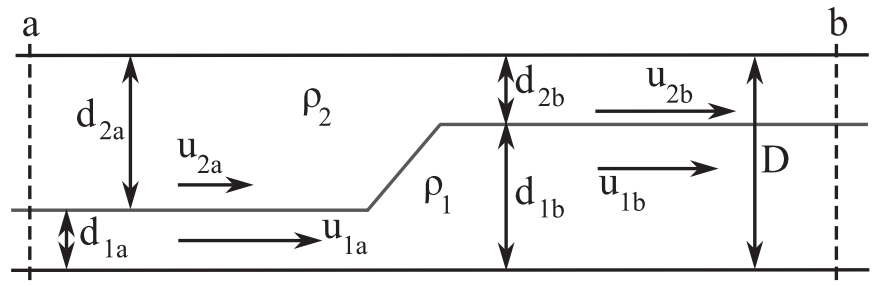

Figure 2-1: Idealized problem setup.

\subsection{Two-layer theories}

The two-layer flow setup is shown in figure 2-1. Two immiscible layers of distinct densities, $\rho_{i}$, flow between rigid horizontal boundaries separated by a constant distance $D$. The lower layer undergoes a sudden expansion, or hydraulic jump. In this work, the lower layer is always taken to be shallower upstream and thus the expanding layer. However, for Boussinesq flows, the results also apply to hydraulic jumps with expanding upper layers. Each layer has a depth, $d_{i}$, and velocity, $u_{i}$, which change across the jump. The jump is considered in the frame of reference moving with the jump, so the layer velocities are relative to the speed of the jump and the governing equations are stationary. The subscript $i=1$ (2) indicates the lower (upper) layer. The subscript $a(b)$ indicates upstream (downstream) of the jump, corresponding to the vertical sections similarly labeled in figure 2-1.

The two-layer theories explored by Wood and Simpson (1984), Klemp et al. (1997), and Li and Cummins (1998) are shock-joining models based on conservation of layer mass and total momentum flux across the jump (c.f., Klemp et al., 1997),

$$
\begin{aligned}
& {\left[d_{i} u_{i}\right]_{a}^{b}=0, \text { for } i=1,2,} \\
& {\left[\int_{0}^{D}\left(p+\rho u^{2}\right) d z\right]_{a}^{b}=0}
\end{aligned}
$$


where $[\cdot]_{a}^{b}$ indicates the difference across the bore (downstream minus upstream). The volume flow rate per unit width in each layer is $q_{i}=d_{i} u_{i}$ and $p(z)$ is the pressure.

The theories assume that there is no mixing, so the density in each layer is constant, and the detailed dynamics within the jump are ignored. The problem is considered in the frame of reference moving with the bore. If the upstream conditions are known, then these three equations should allow one to solve for the downstream conditions, $u_{1 d}, u_{2 d}, d_{1 d}, d_{2 d}$, using the rigid-lid assumption, $d_{1}+d_{2}=D$, and assuming the pressure is hydrostatic in the uniform regions up- and downstream of the jump:

$$
p= \begin{cases}p_{s}+g \rho_{2}(D-z) & , d_{1}<z<d_{2}, \\ p_{s}+g \rho_{2} d_{2}+g \rho_{1}\left(D-d_{2}-z\right) & , 0<z<d_{1} .\end{cases}
$$

However, the surface pressure, $p(z=D)=p_{s}$, is unknown and can change across the jump. This introduces an additional unknown, $\left[p_{s}\right]_{a}^{b}$, requiring another constraint to close the system. This change in surface pressure in a rigid-lid system is equivalent to a change in surface elevation in a free-surface flow. To calculate the change in surface pressure, Wood and Simpson (1984) assumed conservation of energy flux in the upper (contracting) layer, which is equivalent to conserving the Bernoulli function along $z=D$. With this assumption,

$$
\left[E_{2}\right]_{a}^{b}=\left[\int_{d_{1}}^{D} u\left(p+\rho \frac{u^{2}}{2}+\rho g z\right) d z\right]_{a}^{b}=q_{2}\left[p_{s}+\rho_{2} u_{2}^{2}\right]_{a}^{b}=0
$$

and

$$
\left[p_{s}\right]_{a}^{b}=-\left[\rho_{2} u_{2}^{2}\right]_{a}^{b} .
$$

After eliminating $u_{2 b}$, the closed set of equations for the unknown $d_{2 b}$ are

$D\left[p_{s}\right]_{a}^{b}+\left[\left(\rho_{1}-\rho_{2}\right) \frac{g}{2}\left(d_{1 b}^{2}-d_{1 a}^{2}\right)+\rho_{1}\left(\frac{q_{1}^{2}}{d_{1 b}}-u_{1 a}^{2} d_{1 a}\right)+\rho_{2}\left(\frac{q_{2}^{2}}{D-d_{1 b}}-u_{2 a}^{2} d_{2 a}\right)\right]=0$

and

$$
\left[p_{s}\right]_{a}^{b}=\frac{\rho_{2}}{2}\left[u_{2 a}^{2}-\left(\frac{q_{2}}{D-d_{1 b}}\right)^{2}\right]
$$


Together, (2.2.6) and (2.2.7) will be referred to as the WS theory.

Klemp et al. (1997) note that the WS theory results in an incorrect bore speed in the gravity-current limit $d_{1 a} \rightarrow 0$. They corrected this by conserving energy flux in the lower (expanding) layer,

$$
\left[E_{1}\right]_{a}^{b}=\left[\int_{0}^{d_{1}} u\left(p+\rho \frac{u^{2}}{2}+\rho g z\right) d z\right]_{a}^{b}=0
$$

to give, after eliminating $u_{2 b}$,

$$
\left[p_{s}\right]_{a}^{b}=\left[g d_{2}\left(\rho_{1}-\rho_{2}\right)-\rho_{1} u_{1}^{2}\right]_{a}^{b}=g\left(\rho_{2}-\rho_{1}\right)\left(d_{1 b}-d_{1 a}\right)+\frac{\rho_{1}}{2}\left[u_{1 a}^{2}-\left(\frac{q_{1}}{d_{1 b}}\right)^{2}\right]
$$

This model will be referred to as the KRS theory. The final equations for $d_{2 b}$, given upstream values of $d_{i}$ and $u_{i}$, are (2.2.6) and (2.2.9).

The KRS theory gives the correct linear long-wave phase speeds and reduces to the Benjamin (1968) two-layer gravity current speed. Klemp et al. (1997) suggest that energy conservation in the expanding layer is most appropriate for jumps with zero or small upstream shear, and jumps with a thin upstream lower layer $\left(\frac{d_{1 a}}{D} \ll 1\right)$. For jumps with large and positive upstream shear $\left(u_{1 a} \gg u_{2 a}\right)$, which typically develop an embedded roller (Wood and Simpson, 1984), Klemp et al. (1997) suggest that the WS closure might be more appropriate.

Borden and Meiburg (2013b) employed an alternative approach for two-layer internal jumps with no upstream shear (i.e., internal bores). By integrating the vorticity equation over the area of the jump they found that the baroclinic vorticity production through the jump equaled the downstream flux of vorticity. Their closure can also be derived directly from the shallow water equations (Helfrich, 1995; Borden and Meiburg, 2013a; White and Helfrich, 2014). The steady, two-layer shallow water 
equations can be written as

$$
\frac{\partial}{\partial x}\left(\frac{u_{i}^{2}}{2}+\frac{p_{i}}{\rho_{i}}\right)=0, \quad \frac{\partial}{\partial x}\left(d_{i} u_{i}\right)=0, \quad i=1,2
$$

where the pressure $p_{i}$ is given by (2.2.3). Using the Boussinesq approximation, assuming a rigid-lid, differencing the momentum equations between the layers to eliminate $p_{s}$, and then integrating the resulting equations across the jump in the usual shockjoining approach gives

$$
\begin{gathered}
{\left[\frac{\Delta u\left(q_{1}+q_{2}\right)+(\Delta u)^{2} d_{2}}{D}-\frac{(\Delta u)^{2}}{2}-g^{\prime} d_{2}\right]_{a}^{b}=0,} \\
{\left[\frac{d_{2}\left(q_{1}+q_{2}\right)+\Delta u d_{2}^{2}}{D}-d_{2} \Delta u\right]_{a}^{b}=0 .}
\end{gathered}
$$

Here $\Delta u=u_{1}-u_{2}$ is the velocity jump between layers, $d_{2}=D-d_{1}$, and $g^{\prime}=$ $g\left(\rho_{1}-\rho_{2}\right) / \rho_{1}$ is the reduced gravity. Given $\Delta u_{a}$ and $d_{2 a},(2.2 .11)$ and $(2.2 .12)$ can be solved for $\Delta u_{b}$ and $d_{2 b}$. In the limit of zero upstream shear, $\Delta u_{a}=0$, the Borden and Meiburg (2013b) model is recovered. Unlike the KRS and WS theories, this approach is derived here using the Boussinesq approximation, which is acceptable for the oceanographic applications in mind. This theory will be referred to as the VS (vortex sheet) model. As noted by Borden and Meiburg (2013a) and White and Helfrich (2014), this closure distributes the dissipation in both layers such that the difference in Bernoulli function between layers is conserved across the jump.

\subsubsection{Solution space of basic two-layer theories}

Prior to discussing the solutions, it is convenient to non-dimensionalize variables using the upstream lower layer depth, $d_{1 a}$, for lengths and $\sqrt{g^{\prime} d_{1 a}}$ for velocities. With this scaling, a two-layer Boussinesq flow (assumed below unless stated otherwise), in the frame of reference moving with the jump, can be fully described by the four 
non-dimensional parameters

$$
U_{0}=\frac{u_{1 a}}{\sqrt{g^{\prime} d_{1 a}}}, \quad s=\frac{u_{1 a}-u_{2 a}}{\sqrt{g^{\prime} d_{1 a}}}, \frac{1}{r}=\frac{D}{d_{1 a}}, \quad R=\frac{d_{1 b}}{d_{1 a}}
$$

where $U_{0}$ is the upstream lower layer velocity, $s$, the velocity difference between layers, $r^{-1}$, the total depth, and $R$ is the downstream lower depth. The velocities are scaled by $\sqrt{g^{\prime} d_{1 a}}$, the lengths by $d_{1 a}$, and time by $\sqrt{\frac{d_{1 a}}{g^{\prime}}}$. Given $r^{-1}$ and $s, R=R\left(U_{0}\right)$ can be found from any of the two-layer theories discussed in $\S 2.2$.

Figure 2-2a shows the full solution space for $R\left(U_{0}\right)$ for the KRS model with $r=0.1$ and $s=1$. However, not all possible solutions are physically relevant. Allowable solutions are determined, in part, by the long wave phase speeds, $c_{ \pm}$, in dimensional variables (Baines, 1995),

$$
c_{ \pm}=\frac{u_{1} d_{2}+u_{2} d_{1}}{D} \pm\left[g^{\prime} \frac{d_{1} d_{2}}{D}\left(1-\frac{\left(u_{1}-u_{2}\right)^{2}}{g^{\prime} D}\right)\right]^{1 / 2}
$$

on either side of the jump. The sub- and super-criticality of the upstream and downstream regions are indicated in figure 2-2. Here $c_{-}<0(>0)$ implies sub-criticality (super-criticality). If the negative characteristics upstream and downstream of the transition converge, a jump is expected to occur. If they diverge, an initially imposed jump will produce a rarefaction wave. Solutions with converging negative characteristics fall into one of three categories: super- to super-critical, super- to sub-critical, or sub- to sub-critical. The behavior of the positive characteristics does not affect the formation of a shock.

While the full solution space of the KRS theory is shown in figure 2-2a, only a limited range of these solutions are physically allowable. To be structurally stable, solutions must have real $c_{ \pm}$and steady jump solutions require converging negative characteristics. Also, the total energy flux must decrease or be conserved across the jump so that

$$
\left[\int_{0}^{D} u\left(p+\rho \frac{u^{2}}{2}+\rho g z\right) d z\right]_{a}^{b} \leq 0
$$



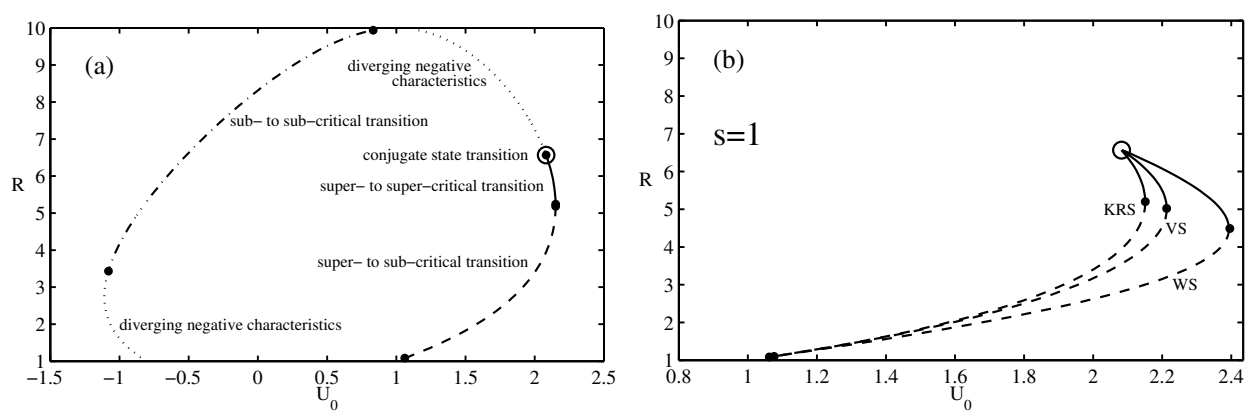

Figure 2-2: (a) Full solution space for the KRS theory with $r=0.1$ and $s=1$. (b) Allowable solution space for KRS, WS and VS theories for $r=0.1$ and $s=1$. Line type indicates behavior of characteristics as marked in (a).
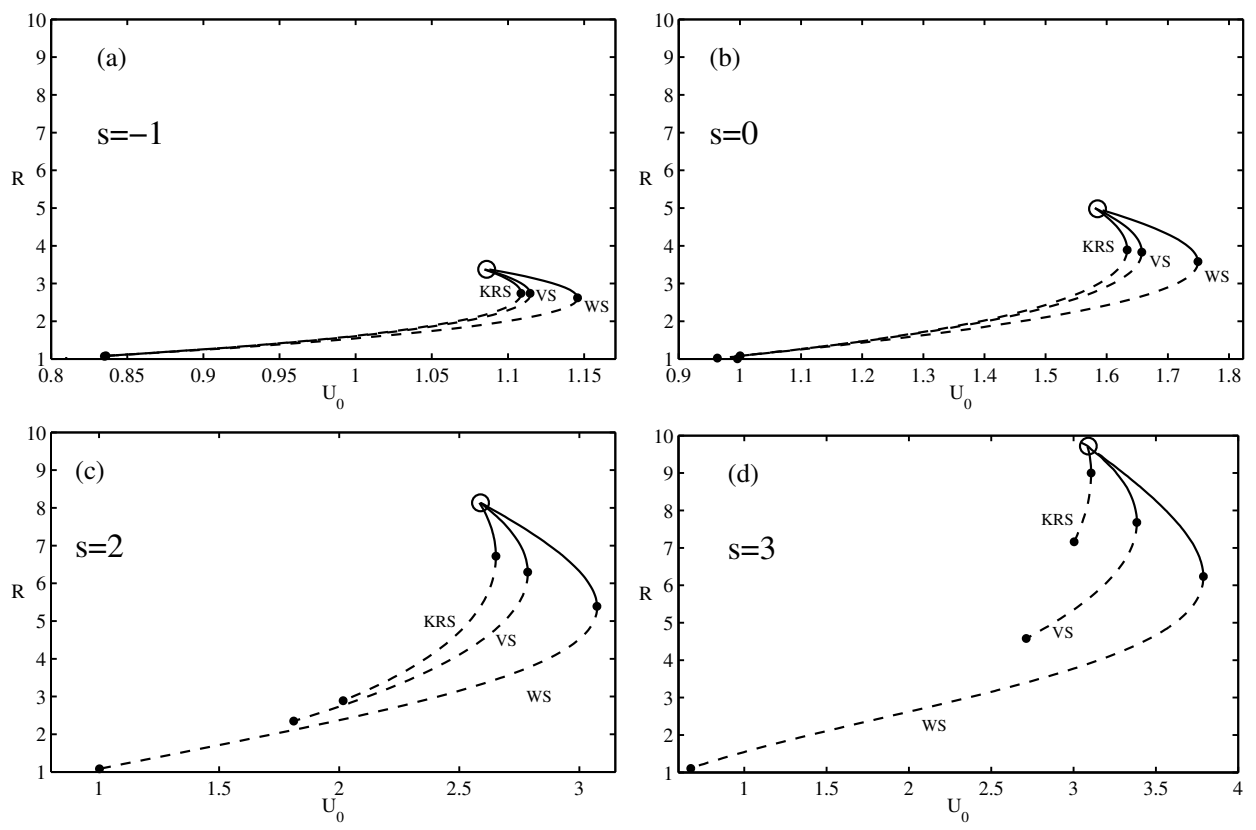

Figure 2-3: (a) Allowable solution space for KRS, WS and VS theories for $r=0.1$ and $s=-1$. (b) Allowable solution space for KRS, WS and VS theories for $r=0.1$ and $s=0$. (c) Allowable solution space for $r=0.1$ and $s=2$, and (d) allowable solution space for $r=0.1$ and $s=3$. Line type indicates behavior of characteristics as marked in figure 2-2(a). 
Finally, $U_{0}$ must be positive to be consistent with the definition of upstream.

The allowable solution spaces of the KRS, VS, and WS theories that satisfy these criteria are shown in figure $2-2 \mathrm{~b}$ for $r=0.1$ and $s=1$. The allowable solutions include super- to sub-critical jumps and super- to super-critical jumps. A special solution, called the conjugate state solution (Lamb, 2000), is also indicated in figure 2-2b. This solution conserves energy in both layers, and the interfacial wave speeds upstream and downstream of the jump are the same. The KRS, WS and VS models all converge to the conjugate state solution. The downstream height $R$ of this special solution is equal to the maximum internal solitary wave amplitude in the given upstream flow (Benjamin, 1968; Stastna and Lamb, 2002). The maximum possible $U_{0}$ occurs at the transition from sub- to super-critical and super- to super-critical jumps. This leads to a range of $U_{0}$ with two possible solutions for $R$.

The allowable solutions for $r=0.1$ with $s=-1,0,2$ and 3 are shown in figures 2-3a to d, respectively. For all theories with the same $s$, the maximum possible $R$, which is the conjugate state solution, is the same. The minimum value of $R(>1)$ also increases for the KRS model, and to a lesser degree for the VS model as $s$ increases. The persistence of small jumps for large $s$ in the WS solutions is consistent with Klemp et al. (1997), who suggest that small jumps with larger shear are better predicted by WS theory, while large jumps with small shear agree more with KRS theory. When the shear increases enough, the models no longer give any allowable solutions. For $r=0.1$, the allowable solution space of the KRS theory extends to $s=3.15$, and for the VS and WS theories, it ends at $s=3.18$. For $r=0.2$, the allowable solution space extends to $s=2.23$ for all three theories. As shear increases, the KRS theory solution space recedes toward the conjugate state solution and then disappears. The two solutions for $R=1$ in the full solution space, shown in figure 2-2a, approach each other as shear increases. They coalesce and then move off the $R=1$ line, at that point the WS and VS theories no longer produce allowable solutions. However, the WS and VS theories maintain a solution curve until this shear value, rather than receding to a single solution as the KRS theory does. As $s$ 
approaches the upper limit for solutions, the maximum allowable jump height (the conjugate state solution) approaches $r^{-1}$.

When $s>0$, there are solutions for $R<1$ (i.e., drops in the lower layer height across the jump). However, these solutions occur for $U_{0}<s$ and are thus exchange flows. Furthermore they have diverging negative characteristics, and would result in a rarefaction wave rather than a hydraulic jump and thus violate our allowable solution criteria.

Without upstream shear, the KRS theory, which conserves energy in the lower (expanding) layer, is more applicable than the WS theory, according to Klemp et al. (1997), although the VS theory, which also dissipates most of the energy in the upper layer, agrees well with simulations in the same parameter range in which the KRS theory works well. Focusing on flows without upstream shear, Borden et al. (2012) recently modified the KRS theory to allow a finite thickness vertical shear layer downstream of the jump that permits momentum and energy transfer from the faster (upper) to the slower (lower) layer. Instead of conserving energy as the KRS theory does, the Borden et al. (2012) modification allows an energy increase in the lower layer. This idea has been extended to flows with upstream shear, although the results are very similar to the KRS theory for large jumps with small upstream shear and to the WS theory for small jumps with large shear, so the remainder of the article focuses on the KRS, WS, and VS theories.

\section{$2.3 \quad$ Numerical modeling}

The theories discussed in $\$ 2.2$ apply to two-layer flows and do not account for the flow within the transition region of the jump that involves nonhydrostatic effects and turbulent mixing. Numerical simulations are thus used to investigate these effects and compared to the two-layer theories to determine which, if any, predicts the behavior of the more realistic numerical simulations. 
The numerical simulations use the non-hydrostatic, adaptive-mesh model IAMR (Almgren et al., 1998). The model solves the incompressible Navier-Stokes equations,

$$
\begin{aligned}
\frac{\partial \vec{u}}{\partial t}+\vec{u} \cdot \nabla \vec{u} & =-\frac{1}{\rho} \nabla p+\nu \nabla^{2} \vec{u}-g \hat{k} \\
\frac{\partial \rho}{\partial t}+\vec{u} \cdot \nabla \rho & =\kappa \nabla^{2} \rho \\
\nabla \cdot \vec{u} & =0 .
\end{aligned}
$$

Here $\vec{u}=(u, v, w)$, with $u$ the along channel, $v$ the transverse (for 3D simulations only), and $w$ the vertical velocities. The density is $\rho, p$ is the pressure, $\nu$ is the kinematic viscosity, $\kappa$ is the density diffusivity, $g$ is the magnitude of gravitational acceleration, and $\hat{k}$ is the unit vector in the vertical direction. The model employs an adaptive Cartesian grid and a time-centered, second-order projection method with a Godunov scheme for the advective terms. The model runs presented here are done in a non-Boussinesq mode required for the open boundaries (see below), although all simulations are essentially Boussinesq with $\Delta \rho / \rho_{1}=0.02$.

IAMR falls into a class of models that have have been termed implicit large-eddy simulation (ILES) models. Because of the non-oscillatory, finite-volume Gudonov treatment of the nonlinear advection terms, the sub-grid stress is in the same form as a viscous stress tensor (Margolin et al., 2006; Grinstein et al., 2007; Aspden et al., 2008). The numerical error acts as a turbulence scheme, dissipating energy at the grid-scale, while conserving mass and momentum, and accurately capturing the energy cascade from resolved scales to the dissipation range at the grid-scale. ILES models have been shown to give solutions that compare very well with DNS and traditional LES models for unstratified turbulent flows (Margolin et al., 2006; Grinstein et al., 2007; Aspden et al., 2008; Zhou et al., 2014), passive-scalar mixing (Hickel et al., 2007) and stratified flows (Waite and Smolarkiewicz, 2008; Remmler and Hickel, 2012). White and Helfrich (2013) found very good agreement of kinetic energy dissipation and irreversible mixing between DNS calculations and ILES solutions with IAMR for intense stratified turbulence in a horizontal shear layer subject to grav- 
itational tilting. These studies support the use of the ILES method for modeling energetic stratified flows such the hydraulic jumps considered here.

The numerical model results are non-dimensionalized in the same way as the twolayer theories and a scaled density

$$
b=\frac{\rho-\rho_{2}}{\rho_{1}-\rho_{2}}
$$

is introduced. Most of the simulations are done in two-dimensions with a domain of $x \in\left[0, L_{x}\right]$ and $z \in\left[0, L_{z}\right]$ where $L_{z}=r^{-1}$ and $L_{x} \gtrsim 10 r^{-1}$. Simulations are initialized with an imposed smooth transition region that adjusts to form a hydraulic jump. In the initial conditions, all of the non-dimensional parameters are set and the transition region, or jump, is stationary. The initial velocity and density fields, in the non-dimensional variables introduced above, vary smoothly in the vertical through a hyperbolic tangent profile with height scale $\lambda^{-1}$ according to

$$
u(x, z, t=0)=\frac{\hat{U}_{0}}{d_{1}(x)}+\frac{\delta u(x)}{2}\left(1-\tanh \left[\lambda\left(z-d_{1}(x)\right)\right]\right)
$$

and

$$
b(x, z, t=0)=\frac{1}{2}\left(1-\tanh \left[\lambda\left(z-d_{1}(x)\right)\right]\right) .
$$

The interface position, $d_{1}(x)$, is given by

$$
d_{1}(x)=1+\frac{\hat{R}-1}{2}\left(1+\tanh \left[\frac{\left(x-x_{0}\right)}{L}\right]\right),
$$

and the velocity jump between the layers,

$$
\delta u(x)=\frac{\hat{U}_{0}}{d_{1}(x)}-\frac{\left(r^{-1}-1\right)\left(\hat{U}_{0}-s\right)}{r^{-1}-d_{1}(x)} .
$$

Here $x_{0}$ is the initial location of the jump and $L$ is the horizontal length scale of the transition. $\hat{R}$ is the initial height of the transition and $\hat{U}_{0}$ is the initial upstream lower layer velocity that are found from a two-layer model solution. The length scales are shown graphically in figure 2-4. 


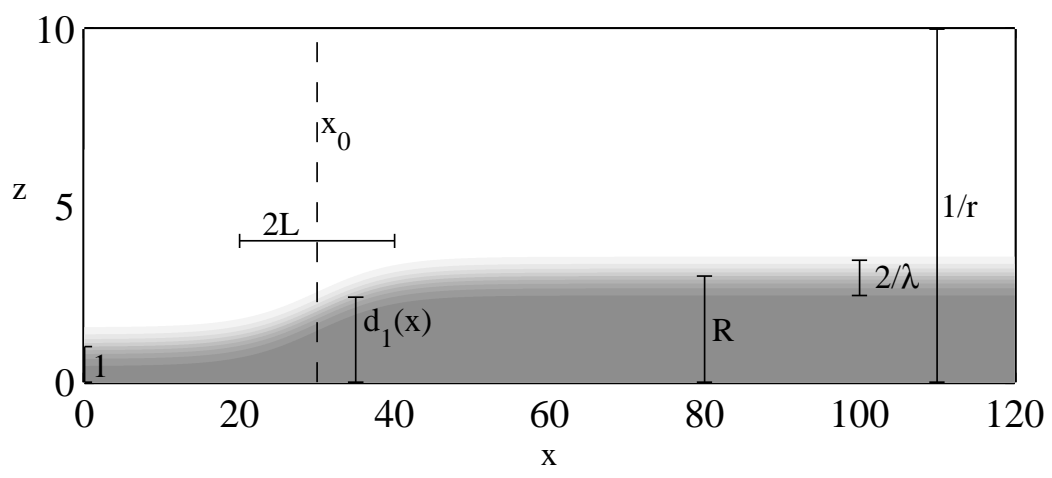

Figure 2-4: Length scales of the flow initialization.

The initial vertical velocity is zero at the top and bottom of the domain and defined so that the flow satisfies the continuity equation,

$$
w(x, z, t=0)=-\int_{0}^{z} \frac{\partial u\left(x, z^{*}, t=0\right)}{\partial x} d z^{*} .
$$

The upstream inlet conditions are given by (2.3.3) and (2.3.4) evaluated at $x=0$. An open boundary condition at $x=L_{x}$, where the density and velocities satisfy Neumann conditions and the pressure is hydrostatic, allows the flow to leave the domain. Free-slip conditions are imposed at the top and bottom boundaries. Thus upstream of the transition, $d_{1}=1$ and $\delta u=s$, while far downstream, $d_{1}=\hat{R}$ and $\delta u$ is given by (2.3.6), which satisfies the two-layer continuity condition. Initial values for $r, s, \hat{U}_{0}$, and $\hat{R}$ are chosen based on the allowable solution space from the two-layer theories. $\hat{U}_{0}$ values from both the KRS and WS theories were tested for a range of $\hat{R}$ values. During a simulation the jump front will usually translate at a small, constant speed and the jump height $\hat{R}$ may also adjust. For comparison with the theories the simulations are shifted into the frame moving with the jump. $\hat{U}_{0}$ is adjusted by the speed of the jump to give $\bar{U}_{0}=\hat{U}_{0}-\bar{U}_{b}$, where $\bar{U}_{b}$ is the time-averaged speed of the jump. Therefore, many of the results presented here were initiated with $\hat{U}_{0}$ values chosen so that the speed of the jump front would be small and the jump would remain in the domain, away from boundaries, for as long as possible. This was achieved by setting $\hat{U}_{0}$ between the KRS and VS theories for large $\hat{R}$, and larger than the WS theory for small $\hat{R}$. 
In the simulation results that will be shown, $\lambda=9, L=3.75, x_{0}=25, \rho_{1}=$ $1+\Delta \rho=1.02$, and $\rho_{2}=1$. The initial interface between the upper and lower layer, parameterized by $\lambda$, is very thin, approximating a two-layer flow.

An isotropic grid with spatial, but temporally stationary, refinement is employed. The low resolution regions are concentrated near the upper boundary and just upstream of the outlet. The latter helps with the open boundary implementation. However, the active jump region is always contained within the finest grid that has an effective resolution of 256 cells in the vertical. The time step varies such that the maximum CFL number $\leq 0.75$. Several tests have been run to ensure that the domain lengths employed are sufficient to prevent the open downstream boundary condition from significantly influencing the results. In most simulations, there is no explicit viscosity or diffusivity $(\nu=\kappa=0)$, so that the effects of any turbulence are handled through ILES. Some simulations with explicit viscosity and diffusivity are conducted, and are discussed further in section 2.3.2.

As an example of the numerical modeling approach, plots of the density field $b$ from a $2 \mathrm{D}$ calculation initiated for $r=0.1, s=1$, and with $\hat{U}_{0}=2.1$ and $\hat{R}=3.7$ model are shown at $t=0,62.6$, and 156.6 in figure 2-5a, b, and c. The initial transition steepens and small-scale shear instabilities quickly form on the downstream interface $(t=62.6)$. The upstream flow has a minimum Richardson number of $R i_{\text {min }}=2 /\left(\lambda \delta u^{2}\right)=0.22$, and the downstream section, where $\delta u=-1.0$ initially, also has $R i_{\text {min }}=0.22$, consistent with the development of downstream shear instability. The upstream section remains smooth because no disturbances are added to the inlet flow and the Kelvin-Helmholtz instability for the weakly unstable inflow has a relatively slow growth rate. The downstream section is disturbed by the steepening bore front, and the downstream wave and flow velocities are slower, allowing disturbances to grow before propagating out of the domain. Coherent vortices are subsequently shed in the lee of the bore front and are swept downstream, dominating the downstream interface so that the small scale shear instabilities are no longer apparent $(t=157)$. The bore front moves slowly downstream at a constant speed. 
The time-averaged density field of the fully developed jump in this steadily moving reference frame of the bore front is shown in figure 2-5d, averaged from $t=157-189$. Note that this average plot has the bore front arbitrarily shifted to $x \approx 10$. For these parameter values, the quasi-steady jump has a smooth front followed by a turbulent interfacial region. The details of the jump structure are discussed more in the next section.

To compare the numerical model results to the two-layer theories, the four nondimensional parameters of the fully adjusted flow, $r, s, \bar{U}_{0}$, and $\bar{R}$, are needed. The initial upstream conditions define $r$ and $s$, which do not change as the flow develops. To determine $\bar{R}$, the interface is first located as follows. At every $x$, the interface is defined so that the vertical integral of the density profile from the simulation is equal to the vertical integral of a two-layer density profile with interface height $\tilde{R}(x)$,

$$
\tilde{R}(x)=\int_{0}^{r^{-1}} b(x, z) d z .
$$

The interface depth $\tilde{R}(x)$ downstream of the jump is then averaged over a region where the flow is approximately horizontally uniform to give a final value, $\bar{R}$. This region excludes the jump front and the part of the domain just upstream of the outlet. The horizontal line in the time-averaged field in figure $2-5 \mathrm{~d}$ indicates the averaging interval. The bore front, identified as the along channel location where the interface height reaches $\tilde{R}=(\epsilon R-1) / 4+1$, is tracked. For jumps with a smooth front, $\epsilon=1.2$, while $\epsilon=1.08$ is used for jumps with a turbulent front. The horizontal velocities are then adjusted into a frame of reference moving with the bore, giving $\bar{U}_{0}$. The simulations are analyzed over a time during which the speed of the bore front is approximately steady, resulting in less than $2 \%$ variation in $U_{0}$ for the smooth front jump shown in figure 2-5. Jumps with a turbulent front have more variation in the speed of the front (approximately 10\% variation in $U_{0}$ ), although the speed oscillates about a constant value and does not exhibit a trend.

This procedure allows each simulation to be directly compared to the two-layer 

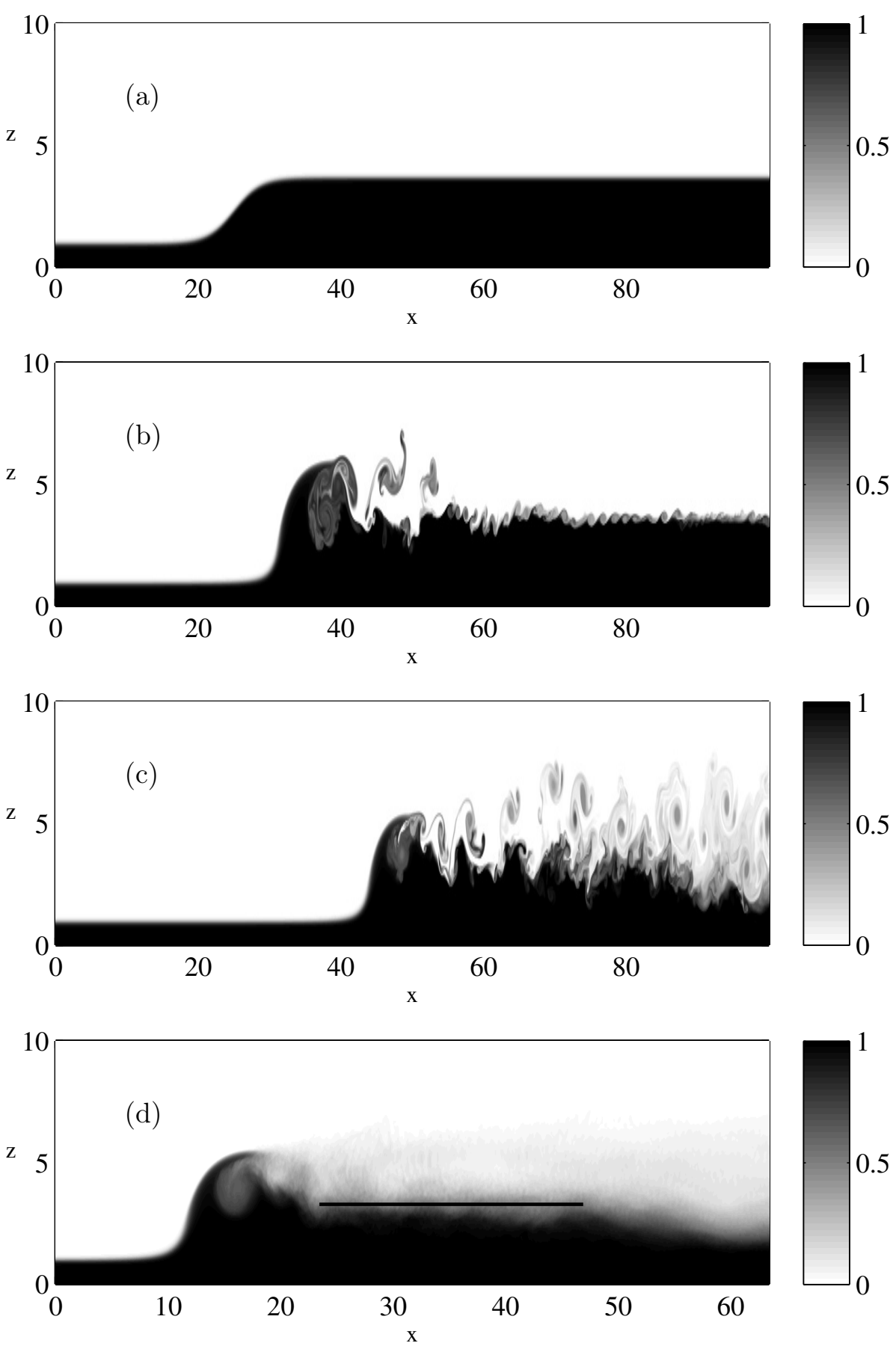

Figure 2-5: Example 2D numerical solution for an initial bore with $r=0.1, s=1, \hat{U}_{0}=2.1$, and $\hat{R}=3.7$. The panels show the instantaneous density field $b$ at (a) $t=0$, (b) $t=62.6$, (c) and $t=157$. The average density field of the fully developed jump, in a frame of reference moving with the bore, is shown in (d). The fully developed jump has $r=0.1, s=1$, $\bar{U}_{0}=1.99$, and $\bar{R}=3.28$. 
theories. For the solution shown in figure 2-5, this gives $\bar{U}_{0}=1.99$ and $\bar{R}=3.28$. Recall that the initial conditions of the simulation were $\hat{U}_{0}=2.1$ and $\hat{R}=3.7$, so that the numerical solution evolved to a slightly slower and smaller jump than was used for the initial conditions.

A large number of 2D simulations, and a few 3D runs (discussed separately in $\S 2.5)$, have been carried out for $r=0.1$ and $s=0-2$ to explore the jump structure and the mean downstream density and velocity fields. A smaller set of simulations with $r=0.2$ have also been conducted, but the calculations for $r=0.1$ are illustrative of results and are the focus of the effort.

\subsubsection{Solution types}

For flows with positive shear $(s>0)$, four distinct, qualitatively different jump types were found in the numerical simulations. They are undular bores, smooth front turbulent jumps, fully turbulent jumps, and conjugate state transitions. Undular bores (UB) consist of a train of solitary-like waves, with the lower layer depth increasing slightly after each wave up to the uniform downstream level, as shown in figure 2-6a. The corresponding velocity field, shown in figure 2-6b, is approximately parallel to isopycnals. There is very little mixing. Consequently, energy loss across the front is due to wave radiation as the train of solitary-like waves spreads with time.

Turbulent jumps may be smooth front turbulent jumps (SFTJ), with a smooth wave-like front, as shown in figure 2-6c and d. In these jumps, the velocity along the bore front is in the upward and downstream direction, with a small counterclockwise recirculation region (vorticity the same sign as the upstream shear layer) slightly downstream of the front. Alternatively, turbulent jumps may be fully turbulent jumps (FTJ) with an overturning leading edge, as shown in figure 2-6g and h. The velocity field figures show that the mean jump consists of two counter-rotating recirculation regions. In both SFTJ and FTJ cases, the interface region downstream of the jump is turbulent with substantial vertical mixing of density and momentum. 
The fourth type are conjugate state (CS) jumps. These are smooth wave-like transitions, which are, in theory, energy conserving. This type of jump is very close to the theoretical conjugate state transition discussed in section 2.2.1, and corresponds to the maximum allowable height of the 'table-top' internal solitary wave (Grimshaw et al., 2004). Numerical simulations of this type of transition exhibit a steady form with very little mixing immediately downstream of the front, which is consistent with energy conservation. For flows without upstream shear, the layer depths downstream of the jump have equal thickness. As shear is increased, the downstream lower layer depth exceeds half of the total depth (Stastna and Lamb, 2002). An example of a conjugate state solution with some upstream shear is shown in figure 2-6e and $\mathrm{f}$. Within and immediately downstream of the front, the velocity is approximately along isopycnals, resulting in very little mixing, consistent with the theoretical conjugate state solution. However, downstream shear instabilities do eventually emerge. These instabilities appear to trip a secondary hydraulic drop from the super-critical flow immediately downstream of the front to a sub-critical state at $x \approx 30$. This super- to sub-critical drop is considered to be a separate feature from the upstream CS transition and may be linked to the presence of an imperfect outflow boundary.

The type of jump that occurs depends on the final jump height $\bar{R}$ (which depends monotonically on $U_{0}$ ) and the upstream shear $s$, for a given $r$. Figure 2-7 shows jump type regime diagrams for $r=0.1$ and $r=0.2$. Small jumps or jumps with small $s$ are undular bores. Large jumps with $s \leq 1$ near the theoretical, two-layer CS solution are conjugate state jumps. Increasing $s$ for moderate $\bar{R}$ gives smooth front turbulent jumps, and further increases in $s$ lead to fully turbulent jumps at all jump heights. For larger $s$, the conjugate state solutions are lost due to intense interfacial instabilities that develop. The distribution of jump types changes with $r$, although the same jump types and the trends described above are maintained.

An interesting behavior is found for moderate shear values near $s=1$.1. Smaller jumps with $\bar{R}<2.5$ are fully turbulent (FTJ), whereas larger jumps are smooth front 

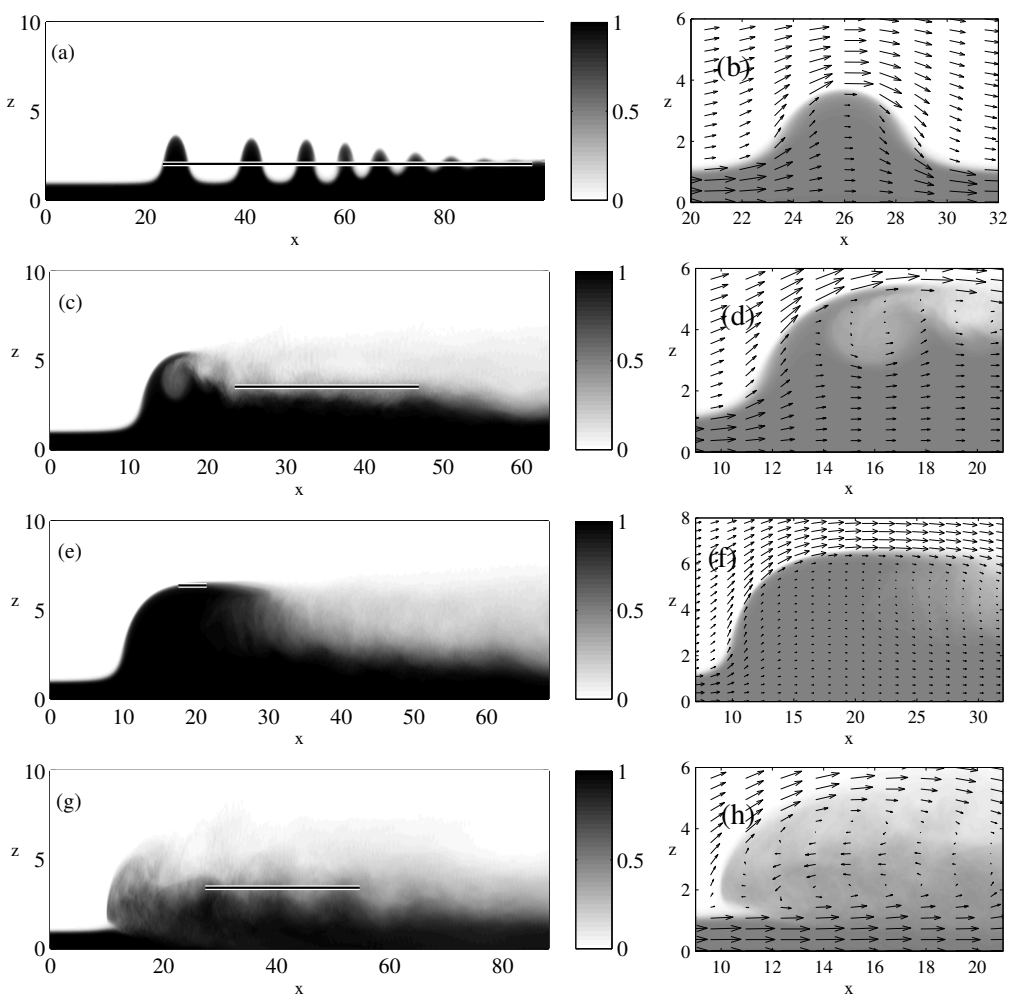

Figure 2-6: The instantaneous density field, $b$, is shown at a non-dimensional time of 157 for (a) an undular bore with $r=0.1, s=1, \bar{R}=2.00$, and $\bar{U}_{0}=1.75$. Time-averaged density fields are shown for (c) a smooth front turbulent jump with $r=0.1, s=1, \bar{R}=3.28$, and $\bar{U}_{0}=1.99$, (e) a conjugate state transition with $r=0.1, s=1, \bar{R}=6.42$, and $\bar{U}_{0}=2.08$, and (f) a fully turbulent jump with $r=0.1, s=1.5, \bar{R}=3.12$, and $\bar{U}_{0}=2.59$. The density field and velocity vectors are shown in the region of the jump in the panels (b), (d), (f), and (h). Note that the density field shown with the velocity vectors uses lighter shades so that the vectors are visible.
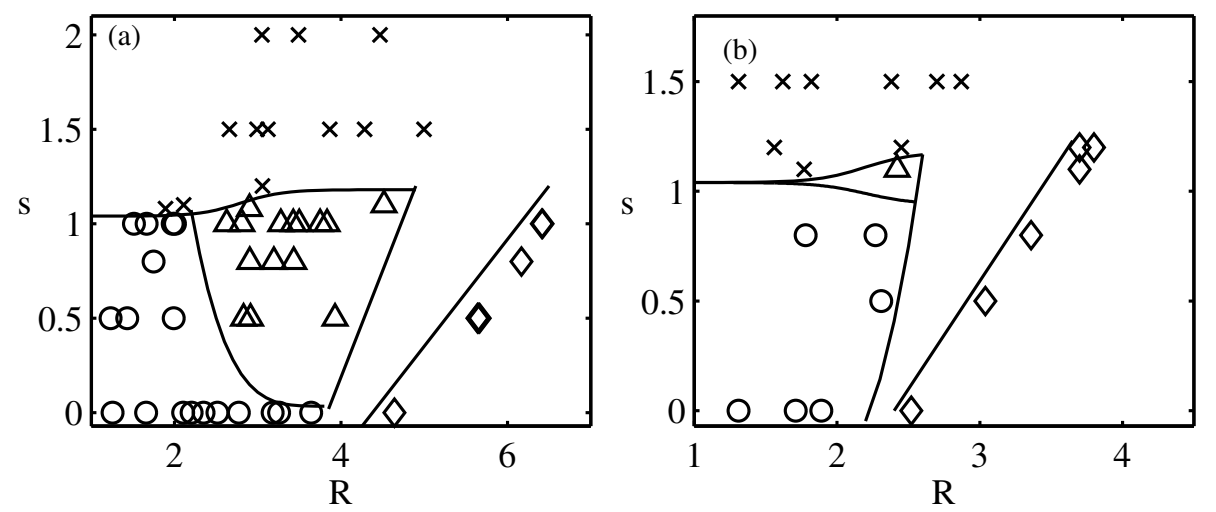

Figure 2-7: The solution type depends on the jump height $\bar{R}$ and the upstream shear $s$. Each point represents a numerical simulation of a given shear and jump height. $\times=$ fully turbulent jump, $\bigcirc=$ undular bore, $\triangle=$ smooth front turbulent jump, and $\diamond=$ conjugate state. (a) $r=0.1$. (b) $r=0.2$. 
turbulent jumps (SFTJ). This is counterintuitive because increased shear and jump height tend to increase instability and turbulence. However, as will be discussed in $\S 2.3 .3$, this unexpected behavior can be linked to the properties of nonlinear waves in the upstream wave guide.

Finally, for a fixed $s \leq 1$, there is a gap between the largest SFTJ or UB and the CS solutions. This gap corresponds to the region of super- to super-critical jumps in the two-layer models shown in figure 2-2. Although these solutions are physically allowable, a numerical simulation initiated in this region will either evolve to a solution near the conjugate state if the initial condition is close enough, or to a jump in the super- to sub-critical region. This gap is further illustrated in the next section where the numerical solutions are compared to the two-layer models.

This work focuses on flows with positive shear $(s>0)$, which have a faster moving lower layer relative to the jump, as this is the type of flow that is generated by topography in locations such as Hood Canal and Knight Inlet. However, as seen in figure 2-3, allowable solutions exist for $s<0$. Because the sign of the upstream vorticity is positive (opposite sign from $s$ ), these solutions are structurally different from those that develop when $s>0$. An example is shown for $s=-1$ in figure 2-8. Jumps with different initial values for $R$ and $U_{0}$ develop similarly. When $s<0$, interfacial instabilities are enhanced because the jump increases the downstream interfacial shear. These instabilities are generally swept downstream, leaving a smooth, solitary wave, or undular bore, type of transition. Overturning at the leading edge of the jump is inhibited by the positive ambient vorticity. Furthermore, jumps with $s<0$ only have allowable solutions for small $R$, which, along with the the direction of the vorticity, helps to explain why they are similar to the undular bores found for $s \geq 0$ (c.f., figure $2-7)$. 

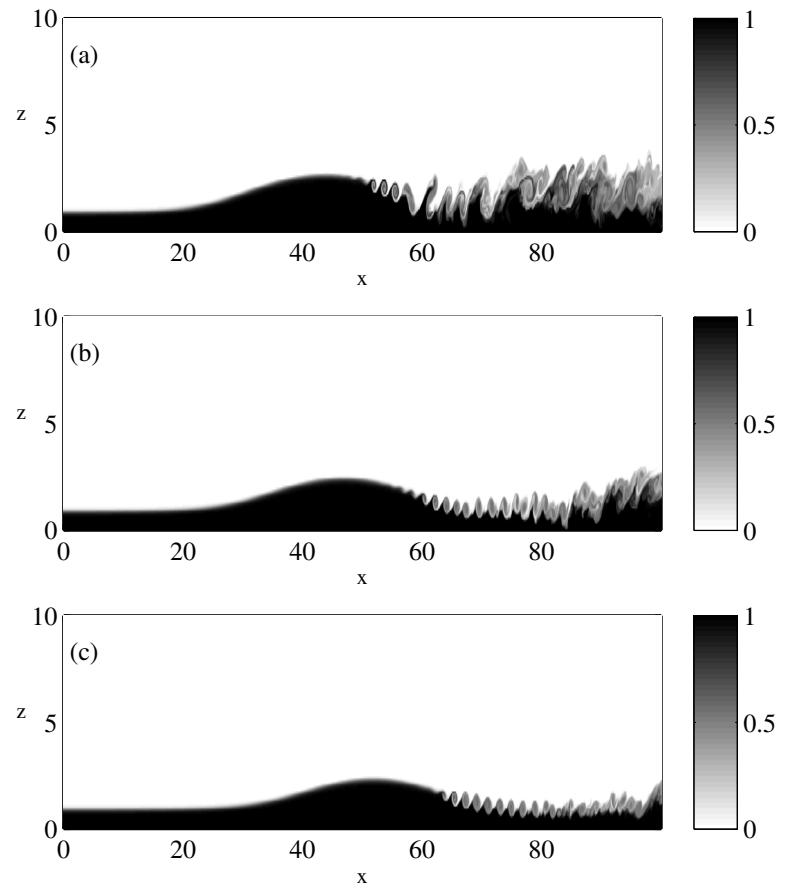

Figure 2-8: The instantaneous density field, $b$, is shown for a flow with $r=0.1$ and $s=-1$ at non-dimensional times of $t=$ (a) 125 , (b) 219, and (c) 313 for initial conditions of $\hat{R}=3.4$, and $\hat{U}_{0}=1.08$.

\subsubsection{Numerical simulations compared to layer theories}

The two-layer theories might be used to predict the approximate height of a stationary $(c=0)$ hydraulic jump, given the upstream layer thicknesses and velocities. Klemp et al. (1997) claim that large jumps without shear should be best predicted by the KRS theory, especially when $r \ll 1$. However, they find that in jumps with upstream shear, the upstream vorticity changes the structure of the jump and the WS theory may be more accurate (Klemp et al., 1997). The VS theory, which replaces specification of the distribution of dissipation with a vorticity conservation condition, gives predictions between the KRS and WS theories.

White and Helfrich (2014) recently developed a theory for flows with continuous stratification without upstream shear, which allows the form of the dissipation to be specified. In the two-layer limit, the form of the dissipation can reduce their theory to any of the three two-layer theories specified above. However White and Helfrich (2014) find that the form that maximizes the total dissipation more accurately 
matches their numerical simulations. This form transfers energy from the upper to the lower layer, an idea that was also used by Borden et al. (2012), who modified the KRS model to transfer energy from the upper to the lower layer.

Simulation results for $\bar{R}$ and $\bar{U}_{0}$ are compared to the KRS, VS, and WS theories with $r=0.1$ and $s=1$ and $s=1.5$ in figures $2-9 \mathrm{a}$ and $\mathrm{b}$, respectively. The qualitative jump type is also indicated. For moderate shear of $s=1$ (figure 2-9a), small jumps $(\bar{R} \lesssim 2)$ agree best with the WS theory, while for larger jumps $(\bar{R} \gtrsim 3)$, the simulations are in better agreement with the KRS theory. The tendency for smaller jumps (and with smaller $\bar{U}_{0}$ ) to agree with the WS model and larger jumps with the KRS model is in broad agreement with the hypothesis of Klemp et al. (1997). Also note that the jump type is an undular bore for $\bar{R} \lesssim 2$ and a smooth-front turbulent jump for larger amplitudes, with the exception of a conjugate state jump for $\bar{R} \approx 6.4$. Jumps initiated in the super- to super-critical region of $4 \lesssim \hat{R} \lesssim 6.4$ develop into either a conjugate state transition if $\hat{R}$ is large enough $(\hat{R} \gtrsim 6)$, or retreat to a smaller super- to sub-critical transition if $\hat{R}$ is smaller. This leaves a gap in the solution space where theoretical super- to super-critical jumps are possible, but numerical solutions do not exist. A possible explanation for this behavior lies in the energy loss associated with each of these branches. For the same $U_{0}$, the downstream sub-critical theoretical solution with a smaller $R$ has more dissipation and less downstream vertical shear than the larger, super-critical solution. This suggests that when two solutions are theoretically possible, the solution that dissipates more energy through the jump is selected.

Although these simulations are bounded by a rigid-lid, the change in pressure at this boundary can be used to estimate the change in surface height that a free-surface flow would experience across a jump. The dimensional change in surface pressure is converted to a non-dimensional change in depth through $\Delta \hat{z}=\frac{\Delta z}{d_{1 a}}=\frac{\Delta p}{\rho g d_{1 a}}$. Because the characteristic pressure in the non-dimensionalization used in this thesis is $\rho g^{\prime} d_{1 a}$, converting a change in surface pressure to a change in surface height involves $\frac{\Delta \rho}{\rho_{0}}$. For the jump shown in figure 2-6c with $\frac{\Delta \rho}{\rho_{0}}=0.02$, the change in surface height would 

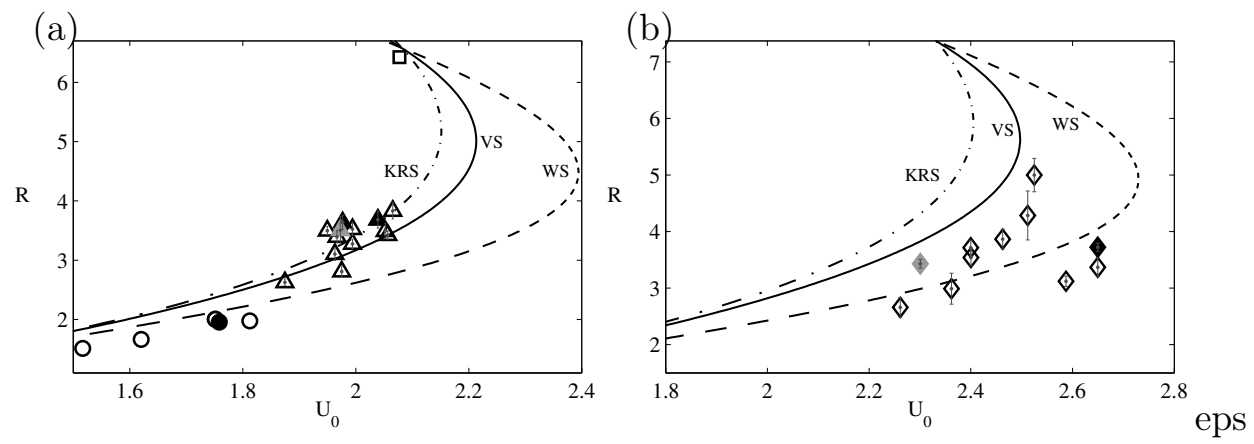

Figure 2-9: (a) Theoretical solution space and numerical simulation results for $r=0.1$ and $s=1 . \square=$ conjugate state transition, $\bigcirc=$ undular bore, $\triangle=$ smooth front turbulent jump. $\bullet=3 \mathrm{D}$ undular bore, $\boldsymbol{\Delta}=3 \mathrm{D}$ smooth front turbulent jump, $\boldsymbol{\Delta}=2 \mathrm{D}$ high-resolution smooth front turbulent jump with $R e=1250$ and $S c=1$. (b) Theoretical solution space and numerical simulation results for $r=0.1$ and $s=1.5, \diamond=2$ D fully turbulent jump, $\diamond=$ $3 \mathrm{D}$ fully turbulent jump, $\nabla=2 \mathrm{D}$ high-resolution fully turbulent jump with $R e=1250$ and $S c=1$. Error bars show one standard deviation of $\tilde{R}(x)$.

be about 0.036 , which is much smaller than the lower layer upstream depth of 1 , indicating that neglecting the change in total depth across the jump is a reasonable approximation. In the oceanic applications of this problem, $\frac{\Delta \rho}{\rho_{0}}$ would be even smaller, resulting in a smaller change in surface elevation.

Simulations with higher upstream shear, $s=1.5$, shown in figure 2-9b, generally fall between the WS and VS predictions. The larger jumps do tend to lie closer to the VS curve, but there is substantial scatter in $\bar{R}$ for the similar values of $\bar{U}_{0}$. All of these jumps in figure 2-9b are the fully turbulent type, which may explain the increased scatter.

Although these simulations give some support to transitions between the two-layer WS and KRS models suggested by Klemp et al. (1997), there is significant scatter in the numerical data, and none of the two-layer theories performs particularly well for the parameter values examined. This is perhaps not too surprising since the twolayer theories neglect flow features such as continuous velocity and density profiles, as well as the dynamics and structure within the jump. Velocity and density profiles, averaged in time and in space downstream of the jump, are shown in figure 2-10a for a smooth front turbulent jump (for $t=157-189$ and $x=23-47$ ) and in 

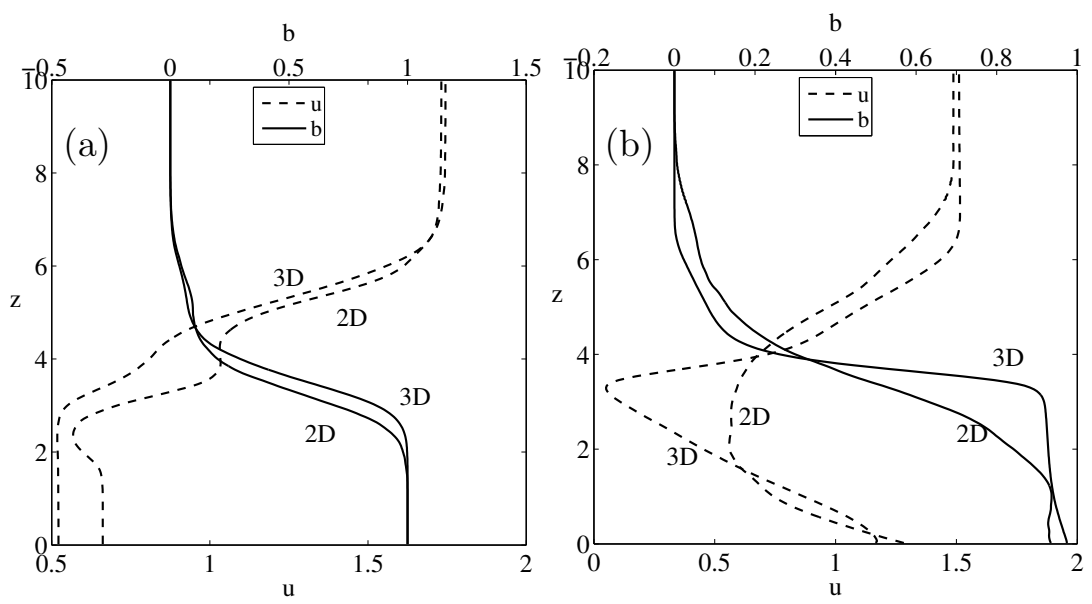

Figure 2-10: Along channel velocity and density profiles downstream of the jump, averaged in space and time. (a) smooth front turbulent jump with $r=0.1, s=1, U_{0}=1.99, R=3.28$ averaged from $t=157-189$ and $x=23-47$. (b) fully turbulent jump with $r=0.1, s=1.5$, $U_{0}=2.59, R=3.12$ averaged from $t=157-189$ and $x=27-55$.

figure 2 -10b for a fully turbulent jump (for $t=157-189$ and $x=27-55$ ). The time-averaged density fields are shown in figures 2-6c and g, respectively. While the downstream density profiles have monotonic variation with height and are reasonably close to two-layered, the velocity profiles exhibit a velocity minimum between the layers. It is particularly pronounced for the higher shear, $s=1.5$, fully turbulent jump in figure 2-10b. The velocity minimum occurs in the lee of the bore head, where the streamlines slowly expand to fill the space behind the bore, and the influence of the jump is felt well downstream of the front.

It is also interesting to note that the strongest density gradient is not coincident with the velocity shear layer. For the smooth front turbulent jump in figure 2-10a, the velocity shear layer extends higher than the density gradient, and for the FTJ, the shear layer extends above and below the density gradient layer. This shear in the velocity profile is generated by baroclinic torque in the turbulent jump head region. These velocity and density profiles are very different from the idealized two-layer theories, and even from the Borden et al. (2012) theory that assumes linear coincident mixing layers downstream of the jumps.

The theoretical models assume no fluid exchange between the layers, i.e. no en- 
trainment. The numerical results show strong vertical mixing and departures from the two-layer assumptions that suggest when viewed in a two-layer limit, entrainment between the layers may occur. This as been assessed from the change in mean lower layer volume flux across the jump

$$
\Delta \bar{q}_{1}=\left[\int_{0}^{\bar{R}} \bar{u}(z) d z\right]_{a}^{b}
$$

Over the range of parameters explored the entrainment, $\Delta \bar{q}_{1} / \bar{q}_{1 a}$, into the lower layer is at most $10 \%$, and generally less than $5 \%$. As discussed in $\S 6$, while entrainment can be incorporated into two-layer jump models, it does not seem to be critical in the parameter range considered here.

The change in energy along streamlines between the downstream averaged state and the upstream state,

$$
\left[\frac{d(\bar{u} \bar{B})}{d \psi}\right]_{a}^{b},
$$

is shown in figures 2-11a and b for the smooth front turbulent jump and the fully turbulent jump in figures 2-10a and b. Here, $B$ is the Bernoulli function and $u$ is the horizontal velocity of the time averaged fields. The derivative with respect to the streamlines, $\psi$, accounts for the spreading of streamlines through the jump, giving a measure of how the distribution of the depth integrated energy flux changes across the jump. The circles indicate the height of the interface downstream of the jump, $\bar{R}$, and the gray bars indicate the average energy flux change across the jump in each layer. In both the SFTJ and the FTJ, there is energy loss in the streamlines both above and below the downstream interface. In the smooth front turbulent jump (figure 2-11a), the streamlines near the interface lose the most energy, while there is a small gain in energy near the lower boundary. In contrast, the streamlines just above the interface of the fully turbulent jump (figure 2-11b) gain some energy, while the lower layer loses energy. In both of these examples, the upper layer moves faster than the lower layer downstream of the jump (see figure 2-10), suggesting that the upper layer might transfer energy to the lower layer downstream of the jump. This transfer 

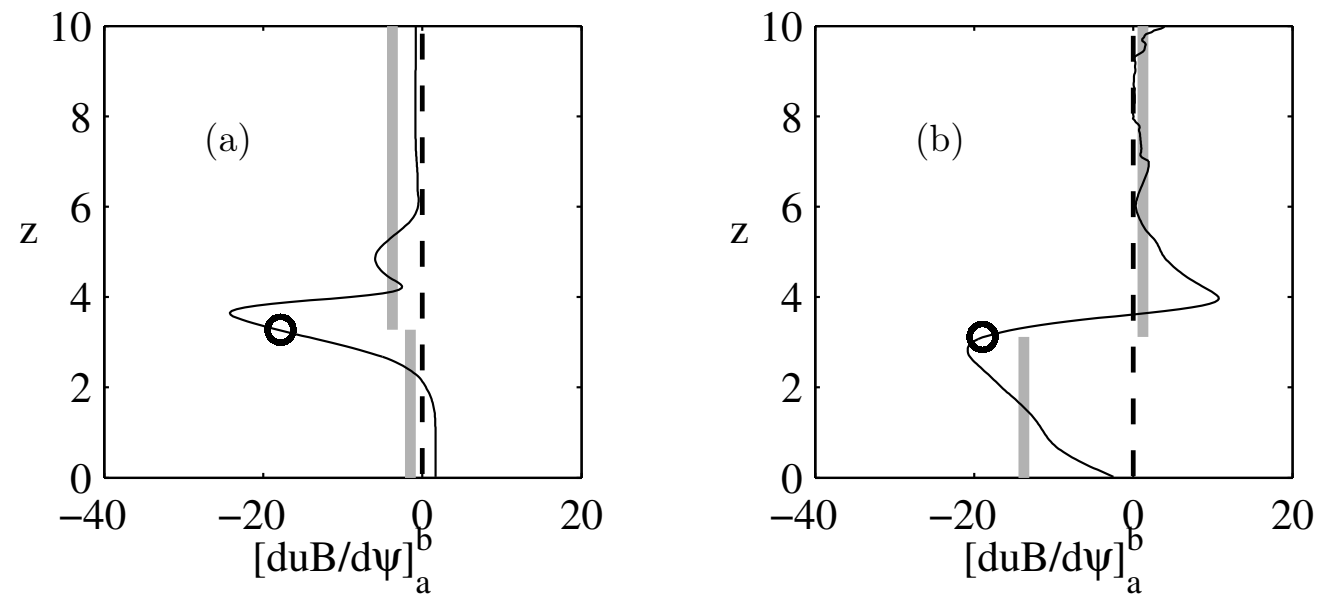

Figure 2-11: Energy flux difference along streamlines versus downstream height of streamline. (a) smooth front turbulent jump with $r=0.1, s=1, \bar{U}_{0}=1.99, \bar{R}=3.28$. (b) fully turbulent jump with $r=0.1, s=1.5, \bar{U}_{0}=2.59, \bar{R}=3.12 . \bar{R}$ is indicated by $\circ$. The average energy flux change in each layer is indicated by the light gray line through the layer.

may be occurring in the smooth front turbulent jump (figure 2-11a) in which there is more energy lost in the upper layer than the lower layer, although it is not evident in the fully turbulent jump (figure 2-11b).

The energy loss in the smooth front turbulent jump peaks in the middle of the velocity shear layer, where there is also a reduced vertical shear. This also corresponds with the upper part of the density gradient zone. In the fully turbulent jump, there are two velocity shear zones, one through the lower part of the flow, with velocity decreasing with height, and another just above the two-layer interface, with velocity increasing with height, and they are separated by a thin layer of low shear. The largest dissipation occurs within this low shear layer, which is also coincident with a region of high density gradient. In both cases, high dissipation coincides with a region of low vertical shear, indicating that the dissipation is tending to stabilize the flow. A similar calculation that includes the mean turbulent turbulent kinetic energy, $\left(\overline{u^{\prime 2}}+\overline{w^{\prime 2}}\right) / 2$, in $\bar{B}$ gives nearly the same result, indicating that the turbulent contribution is negligible. This is expected because the turbulent quantities are much smaller than the means, particularly outside the jump transition region. 
Smooth front turbulent jump heights with $r=0.1$ and $s=1$ are best described by the KRS and VS theories (figure 2-9a). In the KRS model, all of the energy loss is in the upper layer, while in the VS theory, most of the energy loss occurs in the upper layer (approximately 80\% for the SFTJs shown here). The lower layer of the simulated flow loses less energy than the upper layer, consistent with the KRS and VS theories. The fully turbulent jump is better described by the WS theory, which conserves energy in the upper layer, consistent with figure 2-11b. However, in both cases the energy flux distributions of the simulations are much more complicated that the theories allow.

These results suggest, not surprisingly, that the energy changes across hydraulic jumps are not well described by two-layer theories that confine energy loss to a single layer or assume a simple distribution of energy loss and gain. White and Helfrich (2014) calculate theoretical energy loss distributions for jumps with continuous density and velocity profiles and without upstream shear, and showed numerical simulation results for both an undular bore and a smooth front turbulent jump. However, their resulting distributions are not consistent with those in figure 2-11, indicating that upstream shear influences the dissipation distribution. Their equivalent of the smooth front turbulent jump (see their figure 5) exhibits significantly less mixing that is localized further downstream of the bore head than found here.

The vorticity budget can also be analyzed to determine how well the simulations conserve vorticity across the jump, which is the basis of the VS theory (Borden and Meiburg, 2013b). The vorticity budget for a stationary, Reynolds-averaged, Boussinesq, flow integrated over the area of the jump is (in scaled variables)

$$
\left[\int_{0}^{r^{-1}} \bar{u} \bar{\omega}_{2} d z\right]_{0}^{x}+\left[\int_{0}^{r^{-1}} \overline{u^{\prime} \omega_{2}^{\prime}} d z\right]_{0}^{x}-\int_{0}^{x} \int_{0}^{r^{-1}} \frac{\partial \bar{b}}{\partial x} d z d x=\oint_{C} \nu \nabla \bar{\omega}_{2} \cdot \mathbf{n} d s
$$

where $\omega_{2}=\frac{\partial u}{\partial z}-\frac{\partial w}{\partial x}$ is the vorticity in the $y$-direction, $C$ is the contour bounding the control volume, and $\mathbf{n}$ is the outward normal. The overbars indicate mean quantities and the primes denote turbulent departures from the time-mean flow. The first and 
second terms on the left are the divergence of mean and turbulent vorticity fluxes across the region, while the third term is the mean baroclinic production of vorticity within the region. The term on the right is the divergence of the viscous vorticity flux, which will generally be small for high Reynolds number flows. This equation includes the effect of turbulence that may result in vorticity fluxes across the mean streamlines and potentially disrupt the balance between the mean vorticity flux divergence and baroclinic production (terms 1 and 3) assumed by Borden and Meiburg (2013b). We note that these fluxes, if not explicitly included in the budget, could be interpreted as resulting from a turbulent viscosity that would appear in the viscous flux term and are analogous to the production by gradients in (turbulent) dissipation across streamlines in White and Helfrich (2014).

The cumulative integral of each term are shown for a SFTJ in figure 2-12a, and for a FTJ in figure 2-12b. Also indicated in these figures is the downstream region used to find $\bar{R}$, which is also used to determine the downstream averaged values of the vorticity budget terms. The results show that the divergence of the vorticity flux is approximately balanced by the baroclinic production as assumed in the VS model. This is especially true for the SFTJ where the downstream-averaged turbulent fluxes are less than $0.4 \%$ of the mean flux divergence. In the FTJ, the turbulent fluxes are slightly larger, about $6.2 \%$, of the mean flux divergence. In both examples the average residual is positive and comparable $(2.2 \%$ and $7.5 \%$, respectively) to the turbulent flux divergence, indicating that the ILES (numerical) dissipation with the jump region is a small, but non-negligible, contribution to the vorticity budget. These two examples are typical of other runs and indicate that for the parameters considered the vorticity budget integrated over the jump region agrees reasonable well with the mean balance of Borden and Meiburg (2013b).

All of the results presented so far relied entirely on the ILES properties of the numerical scheme to model the grid-scale dissipation and irreversible mixing. In order to assess the ILES calculations, several high-resolution runs with isotropic grids and 1024 cells in the vertical with finite $R e=\left(g^{\prime} d_{1 a}^{3}\right)^{3 / 2} \nu^{-1}=1250$ and $S c=\nu \kappa^{-1}=1$ 

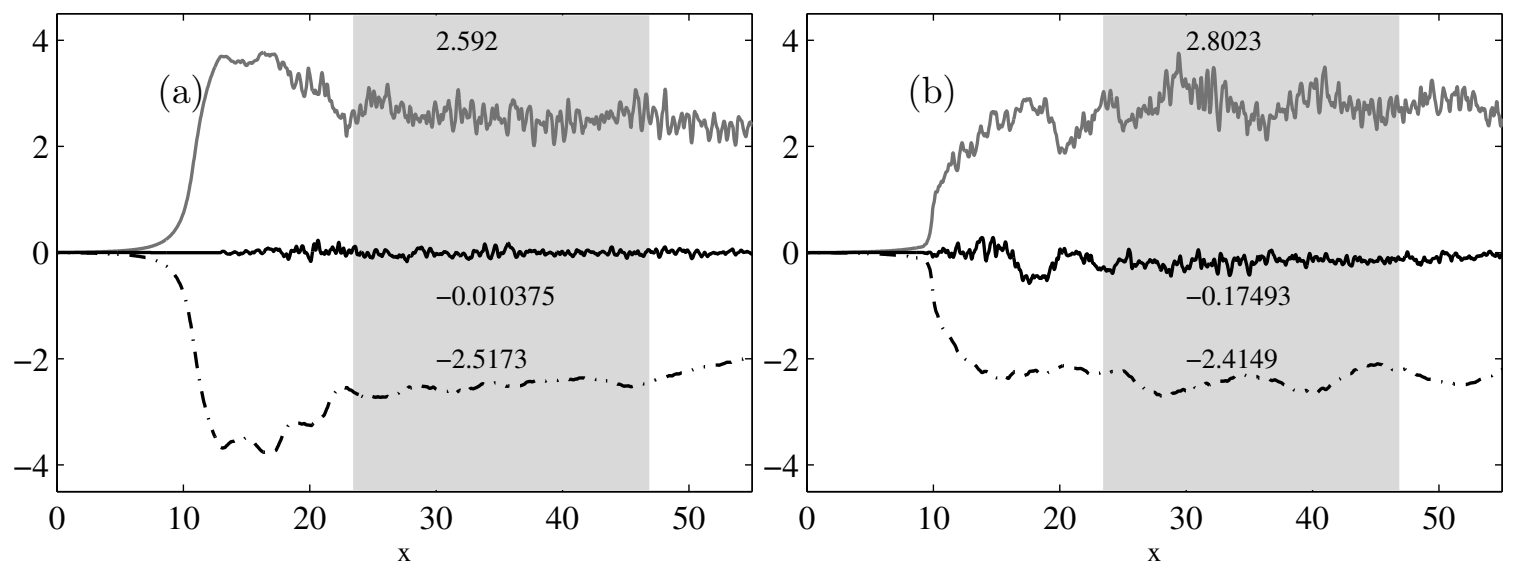

Figure 2-12: Integrated vorticity budget terms for (a) smooth front turbulent jump with $r=0.1, s=1, \bar{U}_{0}=1.99, \bar{R}=3.28$ and (b) fully turbulent jump with $r=0.1, s=1.5$, $\bar{U}_{0}=2.59, \bar{R}=3.12$. - vorticity flux change; - - - baroclinic production of vorticity; turbulent vorticity flux. The dowsntream region is indicated with gray shading, and the average value of each term downstream is also indicated. The average residual for the SFTJ (a) is 0.064 , and for the FTJ (b) is 0.21 .

were conducted. High-resolution runs for the SFTJ and FTJ cases of figure 2-6c and $\mathrm{g}$ are included in figure 2-9 and the results for $\bar{U}_{0}$ and $\bar{R}$ are within a few percent of the ILES values. In these finite-Re runs, over $80 \%$ of the total dissipation is resolved. Futhermore, for the SFTJ, the total dissipation in the high resolution finite Re simulation is very similar to that of the equivalent ILES run, and for the FTJ, the total dissipation of the high resolution run is within a factor of 1.5 of ILES run. While the ILES is a compromise for computational efficiency, these comparisons support the approach as also found by White and Helfrich (2013). The simulation results for $R e=1250$ and $R e \rightarrow \infty$ agree, indicating that the results are not highly dependent on $R e$ in this parameter range. The value of $S c=1$ was chosen because the applications of interest are highly turbulent, such that the turbulent Schmidt number would be approximately one.

\subsubsection{Solitary waves solutions}

Undular bore transitions have so far been classified as hydraulic jumps with the jump front identified as the front of the first wave. However, the simulations show that the wave packet slowly expands and the individual waves separate, acting more like a train 
of solitary waves than a sudden jump. The height of the leading wave might therefore be predicted from the relation between wave amplitude and speed for large-amplitude internal solitary waves obtained from the Dubreil-Jacotin-Long (DJL) model.

The DJL equation for steadily propagating internal solitary waves, in the Boussinesq limit and with an ambient shear flow, is (Stastna and Lamb, 2002)

$$
\begin{gathered}
\nabla^{2} \eta+\frac{U^{\prime}(z-\eta)}{c-U(z-\eta)}\left[\eta_{x}^{2}+\left(\eta_{z}-2\right) \eta_{z}\right]+\frac{N^{2}(z-\eta)}{(c-U(z-\eta))^{2}} \eta=0,0<z<D \\
\eta(x, 0)=\eta(x, D)=\lim _{x \rightarrow \pm \infty} \eta(x, z)=0,
\end{gathered}
$$

where $\eta(x-c t, z)$ is the isopycnal displacement, $N(z)$ is the background buoyancy frequency $U(z)$ is the background shear profile, and $c$ is the speed of the solitary wave. In the frame of reference moving with the leading wave, $U(z)$ is shifted so that $U(z) \rightarrow U(z)-c$ and $c \rightarrow 0$. Simulation results for $\bar{U}_{0}$ can therefore be compared to DJL predictions using $U(0)-c$. The DJL equation is solved numerically using the pseudo arc-length continuation method with Newton-Raphson iteration (LuzzattoFegiz and Helfrich, 2014) with $U(z)$ and $N(z)$ from (2.3.3) and (2.3.4) evaluated upstream. The calculations are initialized with the KdV solution for small amplitude waves and then followed to give the solution curve for $c=c\left(\eta_{\max }\right)$, where $\eta_{\max }$ is the maximum displacement. Each family of solutions ends at the limiting amplitude conjugate state solution. To compare the lead wave of an undular bore to a DJL wave, the maximum height of the leading wave, $\tilde{R}_{\max }$, is obtained. The equivalent DJL wave amplitude is then $\tilde{R}_{\max }-1$.

Results from the numerical simulations are compared to numerical solutions of the DJL equation for $s=0$ and $s=1$ with $r=0.1$ in figure 2-13. It should be noted that in the numerical simulations, the height of the interface downstream of the leading wave is higher than the interface upstream of the wave, whereas the DJL equation assumes symmetry in isopycnal displacement about the center of the wave. Despite this difference, the DJL theory predicts the speed of the leading wave of an undular bore quite accurately. Furthermore, smooth front turbulent jumps, which 


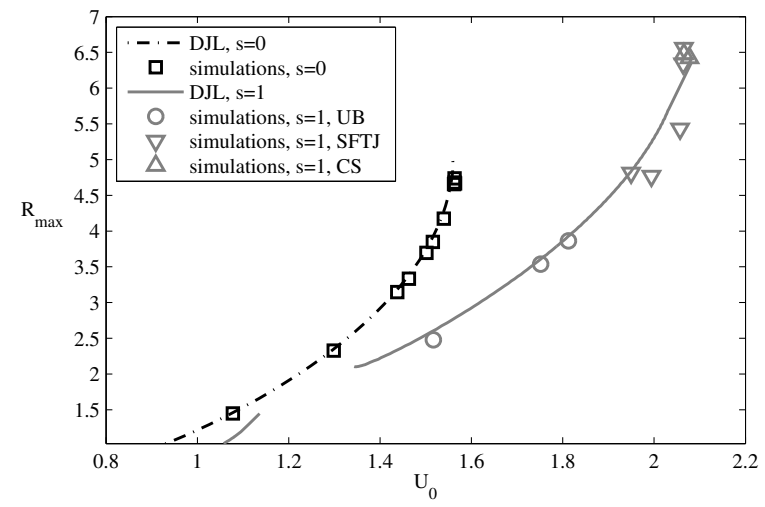

Figure 2-13: Interface height of leading wave $\left(R_{\max }\right)$ versus lower layer upstream speed $\left(U_{0}\right)$ relative to the leading wave. Numerical simulations are compared to convectively stable DJL predictions for $r=0.1$. Note that the $s=1$ curve is discontinuous because convectively unstable solutions that occur for $1.4 \lesssim R_{\max } \lesssim 2.1$, are not plotted.

exhibit complete upstream-downstream asymmetry are also included in figure 2-13 and also show good agreement with the DJL relation $c=c\left(R_{\max }-1\right)$.

DJL solutions for large shear values $(s \gtrsim 1)$ begin to exhibit convective instability for waves with moderate amplitudes (Choi, 2006; Stastna and Lamb, 2002). Convectively unstable solutions develop trapped cores (Stastna and Lamb, 2002; Helfrich and White, 2010; Carr et al., 2012; Luzzatto-Fegiz and Helfrich, 2014). The trapped core solutions are distinguished by regions of closed streamlines with gravitationally unstable density distributions. With ambient shear the recirculation regions may be embedded within the body of the fluid near the interface (Choi, 2006). Streamlines in the recirculation zones do not extend to $|x| \rightarrow \infty$, in violation of the assumptions used to derive the DJL equation. In figure 2-13, convectively unstable DJL solutions develop for $s \gtrsim 1$. Interestingly, the trapped core solutions only appear in the limited amplitude range $1.4 \lesssim R_{\max } \lesssim 2.1$ for $s=1$, and are excluded from the solution curve. Both smaller and larger wave solutions are convectively stable. The intermediate range of convectively unstable solutions coincides with the appearance of fully turbulent jumps, as shown in the regime diagram in figure 2-7a for $s=1.1$ (Note that the regime diagram shows $\bar{R}$, while $\tilde{R}_{\max }$ must be considered in relation to the DJL predictions. Fully turbulent jumps do not have a meaningful $\tilde{R}_{\text {max }}$, and are therefore not included in figure 2-13). These results emphasize the control that the upstream 


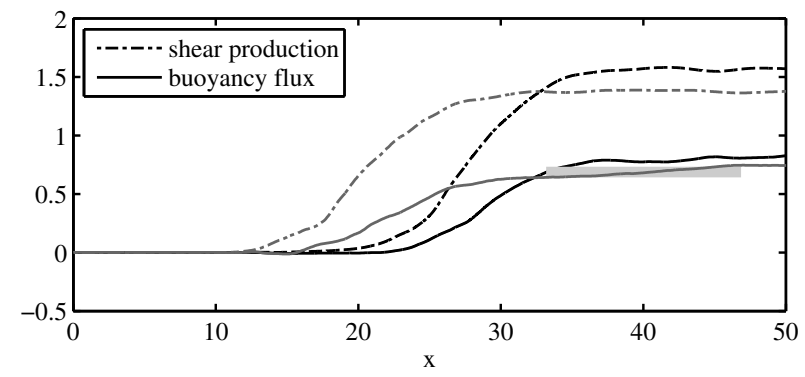

Figure 2-14: Cumulative integral of buoyancy flux and shear production along the domain for the pure ILES simulation with 256 grid points in the vertical with $R e=1250$ and $S c=1$. $r=0.1, s=1, \bar{U}_{0}=1.99$, and $\bar{R}=3.28$ (gray lines) and the fine grid (1024 grid points) simulation with $R e=1250$ and $S c=1$ (black lines). The total mixing, $M$, is indicated for the ILES case by the thick horizontal line.
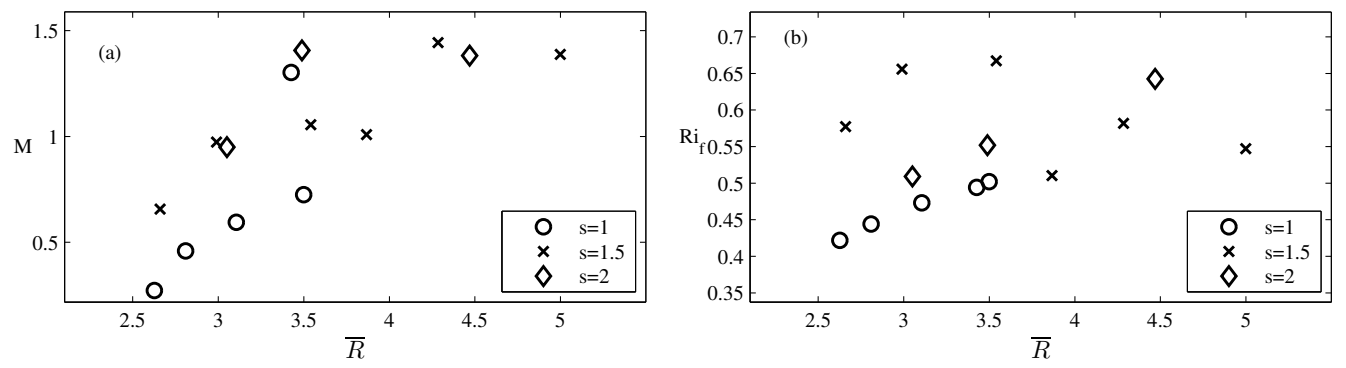

Figure 2-15: (a) $M$ and (b) $\overline{R i_{f}}$ versus $\bar{R}$ for $r=0.1$ and $s=1,1.5$, and 2 as indicated.

wave guide exerts on the bore and jump solutions, in agreement with the results of White and Helfrich (2014).

\subsection{Mixing}

An important consequence of internal hydraulic jumps is turbulent mixing, which dissipates energy and redistributes fluid properties. Mixing can be quantified through the time-averaged turbulent buoyancy flux, $\overline{w^{\prime} b^{\prime}}$, and shear production, $\overline{u_{i}^{\prime} u_{j}^{\prime}} \frac{\partial \bar{U}_{i}}{\partial x_{j}}$, which are terms in turbulent kinetic energy equation (Wyngaard, 2010),

$$
\frac{1}{2} \frac{\partial}{\partial t} \overline{u_{i}^{\prime} u_{i}^{\prime}}=-\frac{U_{j}}{2} \frac{\partial}{\partial x_{j}} \overline{u_{i}^{\prime} u_{i}^{\prime}}-\overline{u_{i}^{\prime} u_{j}^{\prime}} \frac{\partial U_{i}}{\partial x_{j}}-\frac{1}{2} \frac{\partial}{\partial x_{j}} \overline{u_{i}^{\prime} u_{i}^{\prime} u_{j}^{\prime}}-\frac{1}{\rho} \frac{\partial}{\partial x_{i}} \overline{p^{\prime} u_{i}^{\prime}}-\nu \overline{\frac{\partial u_{i}^{\prime}}{\partial x_{j}} \frac{\partial u_{i}^{\prime}}{\partial x_{j}}}-g^{\prime} \overline{u_{3}^{\prime} b^{\prime}} .
$$

The primed quantities are deviations from the time-averaged mean of the fully developed flow (in the frame moving with the bore front). The cumulative area integral of 
the buoyancy flux, which will be referred to as the mixing, $M$, is

$$
M=\int_{0}^{x} \int_{0}^{D} \overline{w^{\prime} b^{\prime}} d z d x
$$

and shear production,

$$
S_{P}=\int_{0}^{x} \int_{0}^{D}-\overline{u_{i}^{\prime} u_{j}^{\prime}} \frac{\partial \bar{u}_{i}}{\partial x_{j}} d z d x,
$$

for the smooth front turbulent jump shown in figure 2-5, with moderate shear of $s=1, \bar{R}=3.28, \bar{U}_{0}=1.99$, and $r=0.1$, are shown in figure 2-14. The simulation is averaged over 151 time steps evenly spaced from $t=156$ to $t=250$. The integral extends through the depth of the flow and from the inlet of the domain to a point $x$ downstream of the inlet. The figure shows that there is an area of intense mixing just downstream of the bore front at $x \approx 12$, leading to a rapid increase in the cumulative buoyancy flux and shear production from $x \approx 15$ to $x \approx 25$. Downstream, the cumulative buoyancy flux and shear production plateau. The average downstream cumulative buoyancy flux is a measure of the total mixing, $M$, of the hydraulic jump. The horizontal extent of the averaging region is the same as used to obtain $\bar{R}$ and is indicated with a horizontal line in figure 2-14.

Figure 2-14 also shows the cumulative buoyancy flux for a fine grid simulation with explicit dissipation. The simulation has 1024 points in the vertical, $R e=1250$, and $S c=1$. The cumulative buoyancy flux and shear production levels are very similar to the pure ILES case, indicating that the ILES approach of approximating turbulence is consistent with simulations in which nearly all of the dissipation is resolved. The region of mixing is shifted downstream in the simulation with a finite $R e$ as a result of a smoother density field in the bore head.

The total mixing, $M$, versus jump height, $\bar{R}$, is shown in figure $2-15$ a for $2 \mathrm{D}$ simulations with several shear values. Shear and jump height both affect the magnitude of mixing. Larger jumps undergo more mixing, and FTJs experience more mixing than SFTJs. The conjugate state jumps, however, are an exception to this trend. These large transitions undergo very little mixing (excluding the subsequent super- 
to subcritical drop), consistent with their energy-conserving properties.

The mixing efficiency,

$$
\overline{R i_{f}}=\frac{1}{x_{2}-x_{1}} \int_{x_{1}}^{x_{2}}\left(\frac{\int_{0}^{x} \int_{0}^{D} \overline{w^{\prime} b^{\prime}} d z d x}{\int_{0}^{x} \int_{0}^{D}-\overline{u_{i}^{\prime} u_{j}^{\prime}} \frac{\partial \overline{u_{i}}}{\partial x_{j}} d z d x}\right) d x
$$

shown in figure 2-15b, does not exhibit an overall trend. The mixing efficiency is slightly higher for FTJs $(s=1.5$ and $s=2)$ than for SFTJs $(s=1)$, but does not increase with shear for a given jump structure. It increases slightly with increasing jump height for SFTJs, but this trend is weak and does not hold for FTJs.

\subsection{Three-dimensional simulations}

Several three-dimensional simulations were carried out to determine whether the results are significantly altered. For all 3D simulations, the initial setup is the same as the corresponding 2D simulation, with the addition of periodic boundary conditions in the transverse, $y$, direction. The width of the domain is one quarter of the height, which limits the development of cross-stream lateral structures in the flow. However, $3 \mathrm{D}$ variations with small length-scales and 3D turbulence can be produced. The grid is isotropic with 256 grid points in the vertical and the simulations are run as pure ILES with zero explicit viscosity and diffusivity.

Instantaneous density surfaces of a $3 \mathrm{D}$ simulation of a smooth front turbulent jump with $r=0.1, s=1, \bar{U}_{0}=2.04$, and $\bar{R}=3.72$ (now also averaged in $y$ ) are shown in figure 2-16a for $t=173$, after the jump has fully adjusted. The initial conditions are equivalent to the $2 \mathrm{D}$ case shown in figure $2-5$. In the $3 \mathrm{D}$ simulation, vortex rolls in the interface downstream of the jump are visible early in the flow development, but subsequently break down rapidly due to $3 \mathrm{D}$ effects, whereas the $2 \mathrm{D}$ simulations develop and maintain more coherent vortex rolls, as shown in figure 2-5c. The jump is otherwise similar to the equivalent $2 \mathrm{D}$ case, with $\left(\bar{U}_{0}, \bar{R}\right)=(1.99,3.28)$ 

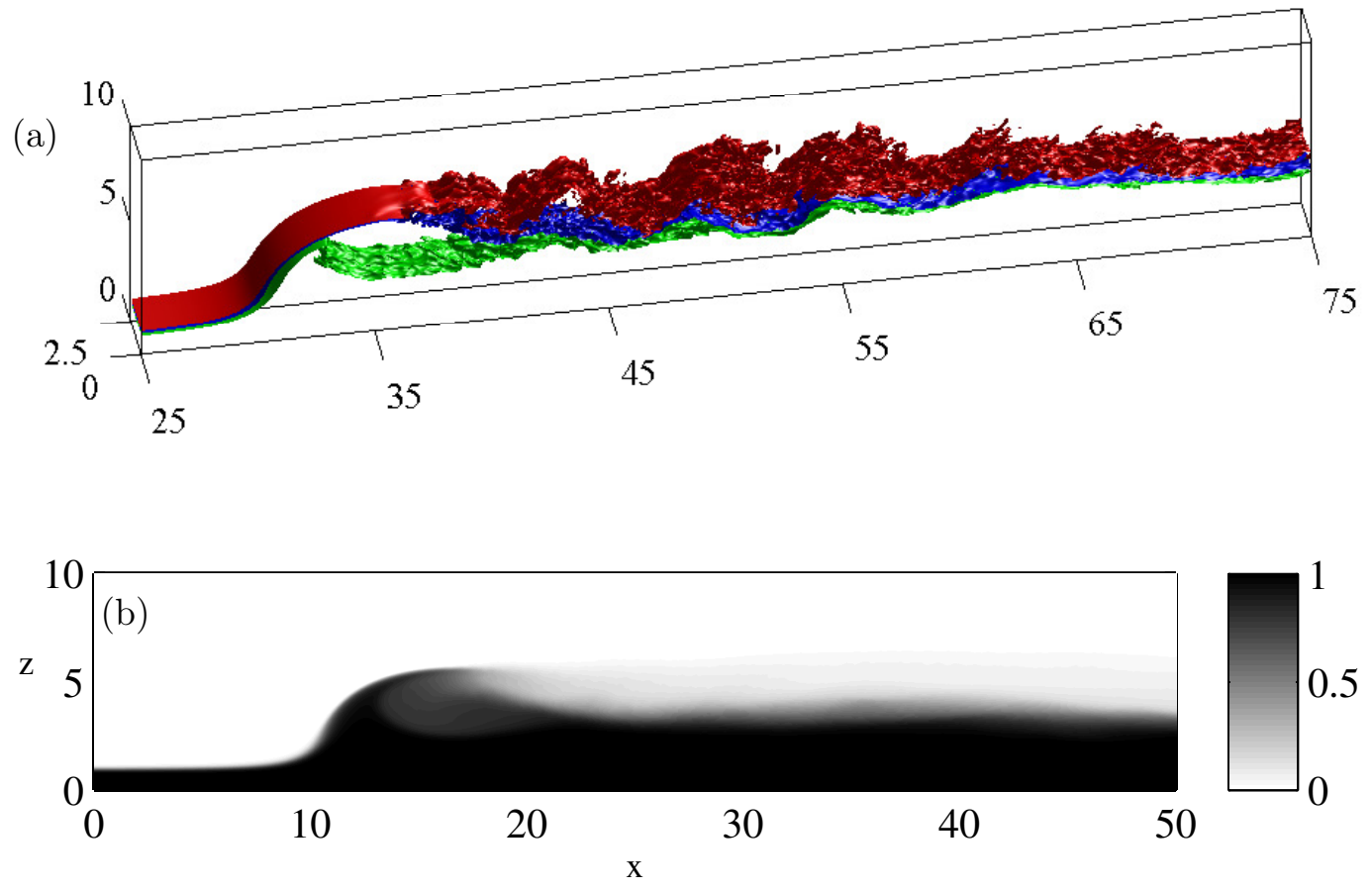

Figure 2-16: (a) Density surfaces of 3D SFTJ. The density surfaces are $b=0.1$ (red), 0.5 (blue), $=0.9$ (green). Simulations parameters are $r=0.1, s=1, \bar{R}=3.72$, and $\bar{U}_{0}=2.04$ shown at $t=157$. (b) Transverse and time averaged density field.

in $2 \mathrm{D}$ and $(2.04,3.72)$ in $3 \mathrm{D}$. The transverse and time averaged density field for the $3 \mathrm{D}$ simulation is shown in figure $2-16 \mathrm{~b}$. It should be compared to the equivalent $2 \mathrm{D}$ case in figure $2-5 \mathrm{~d}$. Both the $2 \mathrm{D}$ and $3 \mathrm{D}$ simulations have been averaged in time from $t=157-188$, with data saved at 51 evenly spaced time steps. The density field from the 3D simulation is much smoother due to the breakdown of the vortex rolls. The downstream-averaged velocity and density profiles (figure 2-10a) are also similar to the 2D case, with a velocity minimum at the bottom of the shear layer and a thicker velocity shear layer than density transition layer.

This 3D smooth front turbulent jump is included in the two-layer theory solution space in figure 2-9a. A 3D smooth front turbulent jump with with the same initial conditions and a domain width of half the total height $(r=0.1, s=1, \bar{R}=3.64$, and $\left.\bar{U}_{0}=1.98\right)$, and a $3 \mathrm{D}$ simulation result for an undular bore $(r=0.1, s=1, \bar{R}=1.96$, and $\left.\bar{U}_{0}=1.76\right)$ are also shown in this figure. For all of these simulations, the 3D results are close to their $2 \mathrm{D}$ companions and within the scatter of the $2 \mathrm{D}$ simulations. 
(a)
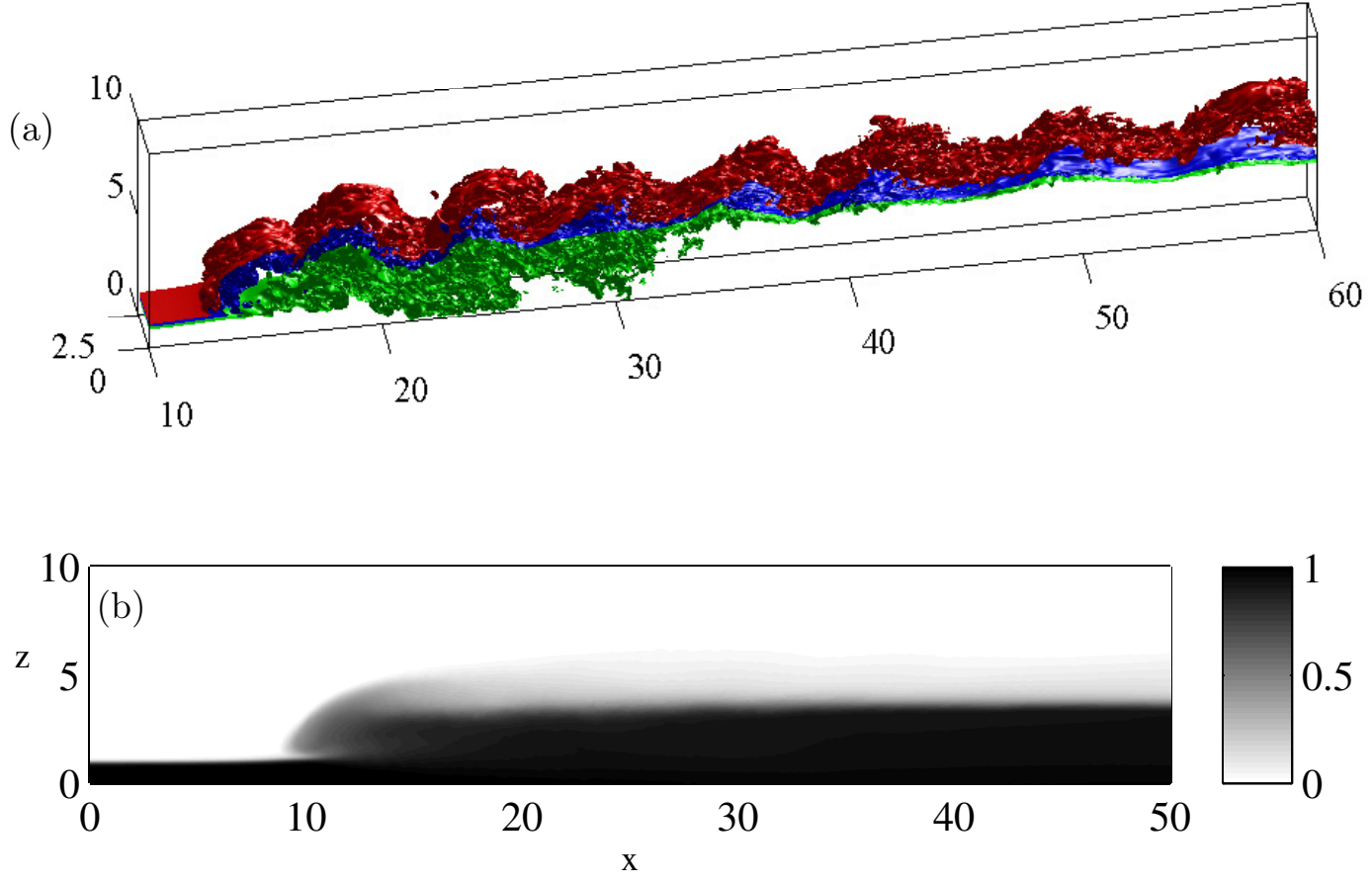

Figure 2-17: (a) Density surfaces of 3D FTJ. The density surfaces are $b=0.1$ (red), 0.5 (blue), $=0.9$ (green). Simulations parameters are $r=0.1$ and $s=1.5, \bar{R}=3.72$, and $\bar{U}_{0}=2.65$. $t=172$. (b) Transverse and time averaged density field.

Instantaneous density surfaces of a fully turbulent hydraulic jump in 3D with $r=0.1, s=1.5, \bar{U}_{0}=2.65$, and $\bar{R}=3.72$ are shown in figure $2-17$ a. The transverseaveraged density field, average in time from $t=157-188$ using 51 time steps, is shown in figure $2-17 \mathrm{~b}$, and should be compared to the equivalent $2 \mathrm{D}$ case in figure 2$6 \mathrm{~g}$ where $\left(\bar{U}_{0}, \bar{R}\right)=(2.59,3.12)$. Downstream-averaged density and velocity fields, shown in figure $2-10 \mathrm{~b}$, are similar to the equivalent $2 \mathrm{D}$ case, with a velocity minimum at a height of $z \approx 3$. The vertical density gradient at the interface between layers is sharper in the 3D result and, while it is not obvious from figures $2-6 \mathrm{~g}$ and $2-17 \mathrm{~b}$, the horizontal density gradient across the bore front is sharper in the 2D case, although the overall properties are otherwise similar. The 3D simulation is compared with the two-layer theories in figure 2-9b. While there are some qualitative differences between the $2 \mathrm{D}$ and $3 \mathrm{D}$ pair, the $3 \mathrm{D}$ simulation falls within the scatter of the $2 \mathrm{D}$ results.

Not surprisingly, vertical mixing in 3D cases is quantitatively different from mixing 


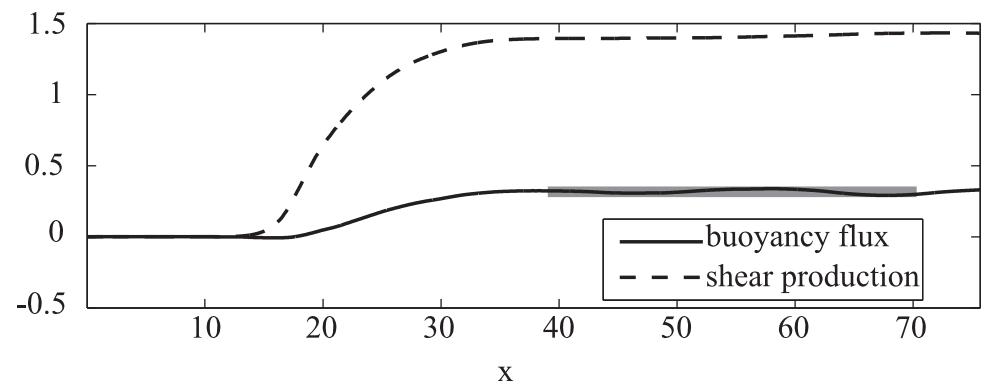

Figure 2-18: Cumulative integrals of buoyancy flux and shear production along the domain from the $3 \mathrm{D}$ run with $r=0.1, s=1, \bar{U}_{0}=2.04$, and $\bar{R}=3.72$. The total mixing $M$, is indicated by the horizontal line.

in the equivalent 2D flows due to vortex breakdown. Shear production of turbulent kinetic energy is consumed by this lateral mixing, reducing the turbulent kinetic energy available for buoyancy fluxes. The cumulative integrals of shear production and buoyancy flux are shown in figure 2-18 for the 3D smooth front turbulent jump with $r=0.1, s=1, \bar{U}_{0}=2.04$, and $\bar{R}=3.72$. This should be compared to the equivalent $2 \mathrm{D}$ case, shown in figure 2-14. The shear production is similar in $2 \mathrm{D}$ and $3 \mathrm{D}$, but because of the energy lost to lateral mixing, the 3D buoyancy flux is about half of the $2 \mathrm{D}$ result, giving reduced mixing efficiency in $3 \mathrm{D}$.

In three dimensions the $y$-vorticity budget (Boussinesq limit) will include vortex stretching terms, $\omega_{i} \partial v / \partial x_{i}$, that could disrupt the balance between mean vorticity flux divergence and baroclinic production assumed in Borden and Meiburg (2013b). However, analysis of the vorticity budgets of these 3D runs gives results similar to the 2D cases in figure 2-12. For the SFTJ the mean vorticity flux divergence is 2.71 (per unit width) and the baroclinic production term is -2.69 . The sum of the mean and turbulent stretching terms is 0.02. The turbulent vorticity flux, 0.02, and residual, 0.04, are both similarly small. The stretching terms, -0.01 , are also insignificant in the FTJ where the primary balance is again between mean flux divergence, 2.96, and baroclinic production, -2.68 . The three-dimensional turbulent flux divergence is now positive, 0.44 , and a slightly larger contributor to the overall balance than in $2 \mathrm{D}$. The residual, 0.74, is also larger, indicating that ILES (turbulent) dissipation is becoming 
important.

\subsection{Conclusions}

The full solution space of three basic two-layer theories for hydraulic jumps with upstream shear was explored. Allowable solutions were found in the super- to sub-critical and super- to super-critical branches of the solution space, including the maximal height, energy conserving conjugate state solution. For some upstream conditions, there are two theoretical downstream states, one of which is a super- to sub-critical transition, while the other is a super- to super-critical transition. When two theoretical solutions are possible, the numerical simulations fall along the super- to sub-critical branch unless they are initialized very close to the theoretical conjugate state solution, in which case they result in conjugate state jumps. The KRS and VS theories were in better agreement with the numerical results for large jumps with smaller shear, while the WS theory agreed better for smaller jumps and larger shear. This is consistent with the predictions of Klemp et al. (1997). However, none of the basic two-layer theories were particularly accurate, and there was significant scatter in the numerical results. Furthermore, the downstream-averaged velocity and density profiles of the numerical simulations were significantly different from the strictly two-layer structure assumed in the theories, and also the linear shear layer modification proposed by Borden et al. (2012). The distribution of energy dissipation was also much more complicated than the two layer theories assume. Together these show that the nonhydrostatic effects and mixing within the jump transition, which are ignored by the two-layer shock-joining theories, are important and need to be accounted for with improved models.

The two-layer theories give solutions only for a limited range of shear values. As shear increases, the allowable solution space decreases, and for large enough shear, no solutions exist. This result also indicates that two-layer theories again may have limited applicability in the presence of significant upstream shear. One modification that 
can be made to the two layer theories to extend the range of solutions for large shear is to include entrainment of fluid from the upper, slower layer, into the faster lower layer following, for example, the approach of Holland et al. (2002). Jacobson et al. (2008) also consider one-and-a-half-layer flow with entrainment by substituting the layer volume conservation equation with energy conservation, and allowing energy that would otherwise be dissipated to generate entrainment. Milewski and Tabak (2015) have explored this approach in two-layer flows, although their model does not include the variation in layer densities across a jump in the momentum balance. This is the topic of ongoing work.

Numerical simulations produced hydraulic jumps of four qualitative forms: undular bores, smooth front turbulent jumps, fully turbulent jumps, and conjugate state jumps. With small shear, small jumps tend to produce undular bores, moderate jump height produce turbulent jumps with smooth fronts, and the largest jumps are conjugate state-like transitions. Increasing the upstream shear results in more turbulent transitions with overturning at the jump front. Undular bores can be treated as hydraulic jumps, but they are perhaps more appropriately described as a train of solitary waves. The DJL equation with a background shear flow can be used to predict the height of the leading wave, given the upstream conditions, including the flow velocity relative to the wave. Solutions to the DJL equation also help explain why, for $s \approx 1$, small jumps can be fully turbulent jumps while larger jumps are smooth front turbulent jumps. This is counterintuitive because larger jumps are generally more turbulent. However, the convectively unstable solutions to the DJL equation for small waves are related to the fully turbulent jumps in the numerical simulations, while the convectively stable DJL solution branch, which exists for larger waves, is associated with smooth front turbulent jumps. As discussed in White and Helfrich (2014) for zero upstream shear, these results highlight the strong influence that the properties of fully-nonlinear wave properties of the upstream wave guide exert on the jumps.

Mixing due to hydraulic jumps was found to occur mainly within a small region 
just downstream of the bore front. The total mixing depends on both the height of the jump and the upstream shear, with more mixing occurring for larger jumps and larger upstream shears. Exceptions to this result are the conjugate state transitions, which are large jumps with very little mixing.

The three-dimensional simulations were compared to results from equivalent 2D simulations, and, despite some qualitative differences, the simulation results agree very well. The bore speed and downstream lower layer depth closely match. This indicates that conclusions drawn from the 2D simulations are likely valid in $3 \mathrm{D}$ for the parameter ranges explored and to the extent that the simple geometry considered here remain applicable.

Examination of the integrated vorticity budgets in both the two- and threedimensional cases supports the Borden and Meiburg (2013b) approach that the mean vorticity flux divergence across a jump is balanced by the mean baroclinic production within the jump. However, there are indications that turbulence, through the turbulent vorticity flux divergence and dissipative effects (through the ILES model), are increasingly important for larger shears and a transition to the fully turbulent jump structure.

This work illustrates a range of structures that hydraulic jumps can exhibit and under which conditions each is likely to occur. It helps explain why undular bores may occur in river outflows, while turbulent jumps occur in peripheral channels such as Knight Inlet. However, further investigation is required to understand jumps in high shear flows where the two-layer solutions are lost, and also for flows with exchangeflow structure with upstream upper layer flow in the opposite direction to the lower layer flow. 
Chapter 3

\section{Internal Hydraulic Jumps with High Upstream Shear}




\subsection{Introduction to high shear jumps}

The allowable and theoretical solution spaces for the WS theory, which conserves energy in the upper layer and is described in chapter 2 , is shown for shear values of $s=0,2$, and 5 in figure 3-1. For shear values of $s \gtrsim 7$, with $r=0.1$, there are no longer mathematical solutions to the KRS, WS, or VS theories. Between shears of 2 and 5 , the two solutions along $R=1$ coalesce and then move off the $R=1$ line. When

this occurs, around $s \gtrsim 3$ for $r=0.1$, there are no longer any allowable solutions, where allowable solutions have real phase speeds at the interface and conserve or dissipate energy across the jump. However, researchers such as Wilkinson and Wood (1971) have shown through experiments that jumps do occur with higher shear. This chapter investigates the physics of these high shear jumps by determining how the theory must be changed to make predictions that are consistent with the jumps produced in numerical simulations. Previous work on high shear jumps, which focuses on one-and-and-half layer flows with a stagnant upper layer, suggests that more mixing may occur and entrainment may be important. Holland et al. (2002), Wilkinson and Wood (1971), and Thorpe (2010) include entrainment in their studies of high shear jumps, and Thorpe (2010) notes that for $F r_{a}>7$ (in the current notation, $U_{0 a}>7$ with $s=U_{0}$ ), entrainment is required for jump solutions to exist, which is consistent with the loss of solutions for the WS theory noted above. However, these studies considered one-and-a-half layer flows with a stagnant upper layer, while here, a two-layer setup with independent upstream shear is considered. Two-layer internal hydraulic jumps with large upstream shear are studied by considering the two-layer theories studied previously, with the addition of entrainment and consideration of the vertical velocity profiles. The theory is compared to numerical simulations, and together, the physical mechanisms that govern high shear jumps are identified.

Although most hydraulic jumps that occur in the environment have lower upstream shear values in the range considered in chapter 2 , it is important to understand how these jumps might change if the upstream conditions are altered (Farmer and Armi, 1999; Gregg and Pratt, 2010; Gregg and Özsoy, 2002). Higher upstream shear 
jumps could develop if the dimensional velocity difference between the two layers, upstream of the jump, is large enough. Alternatively, due to the way the problem is non-dimensionalized, the upstream shear can increase if the density difference between the two layers decreases. Furthermore, this chapter provides some insight into how hydraulic jumps and spatially developing shear flows are related.
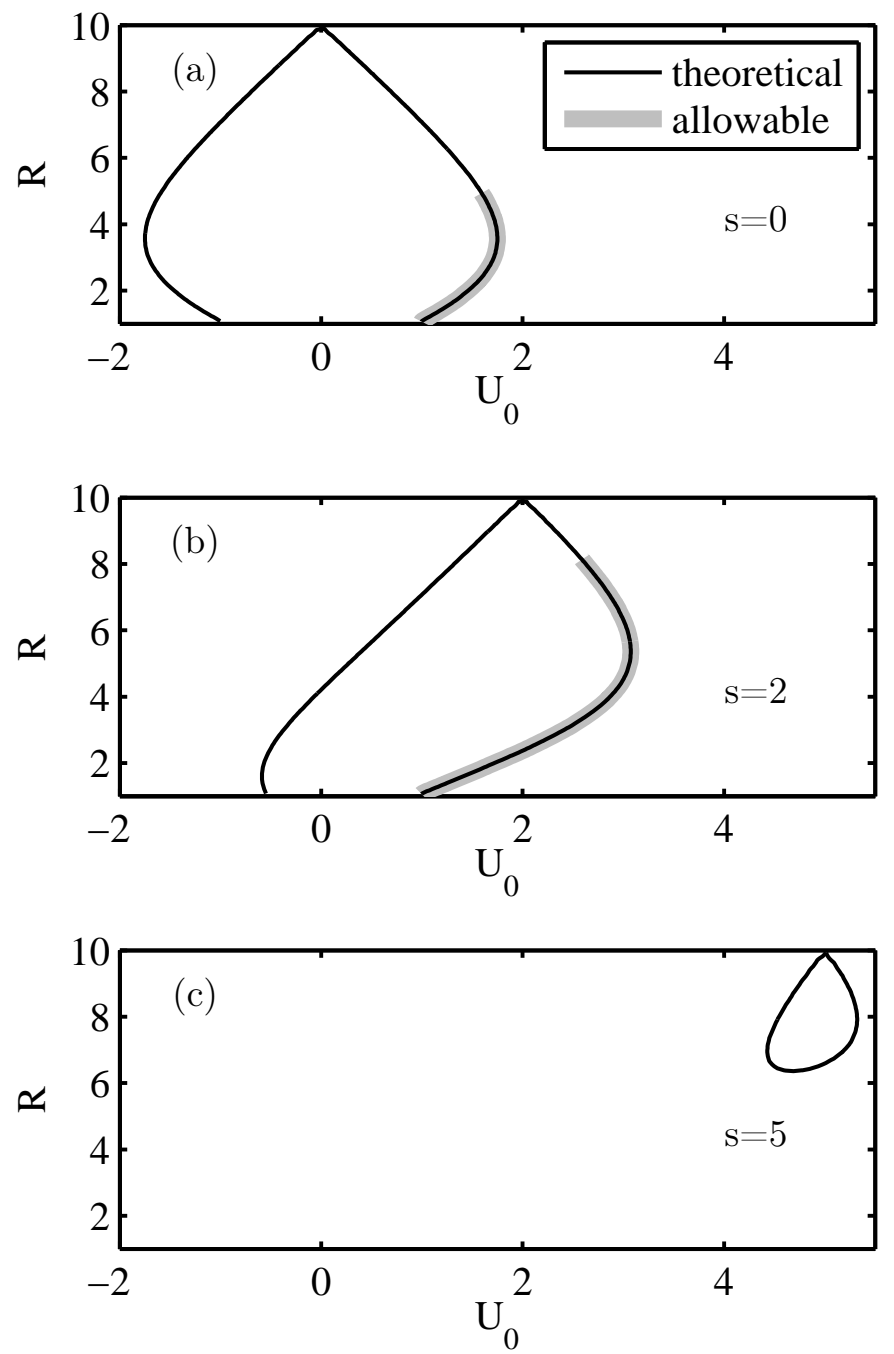

Figure 3-1: Theoretical and allowable solution space for WS theory with various low shear values and $r=0.1$. (a) $s=0$, (b) $s=2$, and (c) $s=5$. 


\subsection{Two-layer theory with entrainment and shape parameters}

In this section, the two-layer theory with entrainment and shape parameters, which quantify the continuous nature of the vertical profiles, are developed. This is different from previous work that includes entrainment such as Wilkinson and Wood (1971) and Holland et al. (2002), who considered one-and-a-half-layer flows with stagnant upper layers. Here, the total depth of the flow is limited, and the upper layer has a velocity that can be non-zero and different from the velocity in the lower layer. The flow setup is shown in dimensional variables in figure 3-2, and non-dimensional variables in figure 3-3. It is non-dimensionalized in the same way as the low shear flows, described in $§ 2.2 .1$. The only additional non-dimensional parameter compared to the low shear flows described in chapter 2 is the entrainment fraction, $q_{\text {efrac }}$, which is the fractional increase in the volume flow rate of the lower layer across the jump. The theory also allows non-dimensional shape parameters, historically referred to as shape functions (Wilkinson and Wood, 1971), which quantify the continuous nature of the velocity profiles. Shape parameters are not actually functions, but rather constants that attempt to account for departures from purely two-layered flow, and are used in the momentum flux equation (3.2.3), as described below.

Entrainment enters into the theory through the volume and mass conservation equations. The volume conservation equation in each layer is modified from that used in the low-shear case to allow a specified entrainment. The entrained fluid is assumed to mix completely throughout the entraining layer, altering the layer's density instantaneously across the jump. The entrainment is positive if the volume flow rate in the lower layer increases across the jump, and negative if the upper layer entrains lower-layer fluid across the jump. The volume conservation equations are

$$
\begin{gathered}
u_{1 a} d_{1 a}\left(1+q_{e f r a c}\right)=u_{1 b} d_{1 b}, \\
u_{2 a} d_{2 a}-q_{e f r a c} u_{1 a} d_{1 a}=u_{2 b} d_{2 b} .
\end{gathered}
$$

Because the density changes across the jump in the layer that entrains fluid, the mass 
conservation equation provides additional information. The mass conservation in the lower (entraining) layer is

$$
u_{1 a} d_{1 a}\left(\rho_{1}+\rho_{2} q_{e f r a c}\right)=\rho_{1 b} u_{1 b} d_{1 b}
$$

If the upper layer entrains fluid from the lower layer, the upper layer density would change across the jump, and the mass conservation equation for the upper layer would be of interest instead. In this chapter, the lower layer is always assumed to be the entraining layer, and this is consistent with the numerical simulations that are described in $\S 3.3$.

The mass (3.2.2) and volume (3.2.1) conservation equations for the lower layer can be used together to calculate the density of the lower layer after the jump, given the entrainment fraction:

$$
\rho_{1 b}=\frac{\rho_{1}+\rho_{2} q_{\text {efrac }}}{1+q_{\text {efrac }}}
$$

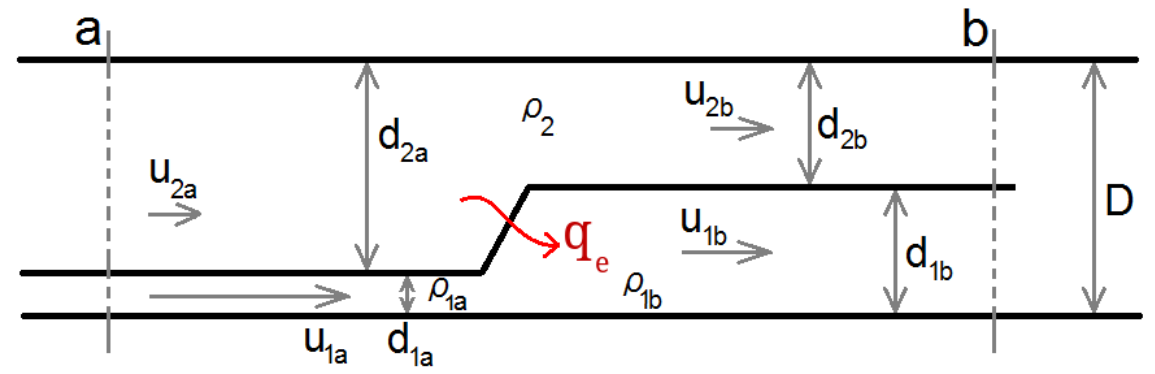

Figure 3-2: Dimensional two-layer flow setup.

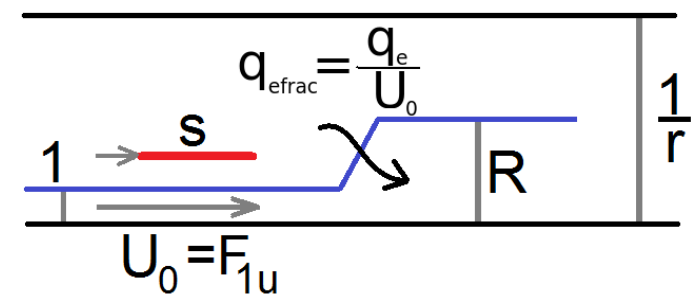

Figure 3-3: Non-dimensional two-layer flow setup with entrainment.

The entrainment does not alter the momentum flux conservation equation, except 
through the density, which changes in the entraining layer across the jump. This also alters $g^{\prime}$, the reduced gravity, across the jump. However, the momentum equation will be modified from the low shear version by the inclusion of shape parameters to represent departures from two-layered flow. The momentum flux conservation equation is

$$
\left[\int_{0}^{D}\left(p+\rho u^{2}\right) d z\right]_{a}^{b}=0
$$

where the pressure is assumed to be hydrostatic away from the jump, and is given by

$$
\begin{gathered}
p(z=D)=p_{s} \\
p= \begin{cases}p_{s}+\rho_{2} g(D-z) & d_{1} \leq z \leq D \\
p_{s}+\rho_{2} g d_{2}+\rho_{1} g\left(d_{1}-z\right) & 0 \leq z \leq d_{1} .\end{cases}
\end{gathered}
$$

Shape functions enter into the momentum flux equation (3.2.3) through the nonlinear terms. Shape functions for a continuous density profile could also be included in the hydrostatic pressure term and in the $\rho u^{2}$ term of the momentum flux equation (3.2.3) (Wilkinson and Wood, 1971). However, the numerical simulations, seen in §3.3.1, show that the density profile usually remains approximately two-layered while the velocity profiles does not, so only shape parameters for the velocity profile are included here. Furthermore, additional shape parameters would require specifying additional unknown parameters to predict a solution. With shape parameters used only for velocity and a constant density in each layer, the $u^{2}$ term in momentum flux equation (3.2.3) is

$$
\int_{0}^{D} u^{2} d z=\int_{0}^{d_{1}} u^{2} d z+\int_{d_{1}}^{D} u^{2} d z .
$$

Considering just the first term on the right hand side, which is the lower layer,

$$
\int_{0}^{d_{1}} u^{2} d z=\frac{\left(\int_{0}^{d_{1}} u d z\right)^{2}}{\left(\int_{0}^{d_{1}} u d z\right)^{2}} \int_{0}^{d_{1}} u^{2} d z=\frac{u_{1}^{2} d_{1}^{2}}{\left(\int_{0}^{d_{1}} u d z\right)^{2}} \int_{0}^{d_{1}} u^{2} d z=u_{1}^{2} d_{1} \frac{d_{1} \int_{0}^{d_{1}} u^{2} d z}{\left(\int_{0}^{d_{1}} u d z\right)^{2}}
$$

If the layered velocities are defined as the layered average of the continuous velocity, 
then

$$
u_{1}=\frac{\int_{0}^{d_{1}} u d y}{d_{1}} ; \quad u_{2}=\frac{\int_{d_{1}}^{d_{2}} u d y}{d_{2}} .
$$

Then, if shape parameters are defined as

$$
S_{1}^{2}=\frac{d_{1} \int_{0}^{d_{1}} u^{2} d z}{\left(\int_{0}^{d_{1}} u d y\right)^{2}} ; \quad S_{2}^{2}=\frac{d_{2} \int_{d_{1}}^{D} u^{2} d z}{\left(\int_{d_{1}}^{D} u d y\right)^{2}},
$$

the momentum flux equation in the two-layer limit with shape parameters for the velocity profiles becomes

$$
\left[p_{s} D+\frac{g}{2} d_{1}^{2}\left(\rho_{1}-\rho_{2}\right)+\rho_{1} S_{1}^{2} u_{1}^{2} d_{1}+\rho_{2} S_{2}^{2} u_{2}^{2} d_{2}\right]_{a}^{b}=0
$$

The justification for shape parameters will be discussed in $§ 3.3 .2$. However, using $S_{1}^{2}=S_{2}^{2}=1$ will reduce the theory to the strictly two-layered limit. It should also be noted that the shape parameters do not specify the shape of the velocity profiles. They simply quantify the deviation from a two-layered profile in a single number. In the Boussinesq limit with shape parameters for velocity, the momentum flux equation (3.2.3) simplifies to

$$
\left[\frac{p_{s}}{\rho_{0}} D+\frac{g^{\prime}}{2} d_{1}^{2}+S_{1}^{2} u_{1}^{2} d_{1}+S_{2}^{2} u_{2}^{2} d_{2}\right]_{a}^{b}=0 .
$$

Note that $\rho_{1}=\rho_{1}(x=a) \neq \rho_{1}(x=b)=\rho_{1 b}$, and $g_{b}^{\prime}=\frac{g_{a}^{\prime}}{1+q_{\text {efrac }}}$.

As with the theories in chapter 2, a closure method must still be used to specify the change in surface pressure across the jump. Because fluid is being entrained from the upper layer into the lower layer and then mixed completely within the lower layer, energy cannot be conserved in the lower layer across the jump. Instead, following Wood and Simpson (1984), energy is conserved along the upper streamline. This is consistent with Klemp et al. (1997), who note that the Wood and Simpson (1984) approach is appropriate for high shear flows. The approximation is usually good, with the change in $B=p+\rho g z+\rho \frac{u^{2}}{2}$ usually significantly smaller along the upper stream line than along the lower streamline. Conserving energy along the streamline 
at $z=D$ to get the change in surface pressure gives

$$
\left[p+\rho_{2} g z+\rho \frac{u^{2}}{2}\right]_{a}^{b}=0 .
$$

Noting that $\rho g D$ is the same at sections a and $\mathrm{b}$, and letting $p_{s}(x=a)=0$, which is allowable because only the change in pressure across the jump is required, gives

$$
p_{s}(x=b)=p_{s b}=\frac{\rho_{2}}{2}\left(u_{2 a}^{2}-u_{2 b}^{2}\right)
$$

Incorporating this result into the momentum flux equation (3.2.5) gives the following system of equations for a Boussinesq flow, which can be solved to fully specify the jump, if the entrainment, $q_{\text {efrac }}$, and the upstream and downstream shape parameters are known:

$$
\begin{gathered}
\frac{D}{2}\left(u_{2 a}^{2}-u_{2 b}^{2}\right)+\left[\frac{g^{\prime}}{2} d_{1}^{2}+S_{1}^{2} u_{1}^{2} d_{1}+S_{2}^{2} u_{2}^{2} d_{2}\right]_{a}^{b}=0 \\
d_{2 b}=D-d_{1 b} \\
u_{1 b}=\frac{u_{1 a} d_{1 a}\left(1+q_{\text {efrac }}\right)}{d_{1 b}} \\
u_{2 b}=\frac{u_{2 a} d_{2 a}-q_{\text {efrac }} u_{1 a} d_{1 a}}{D-d_{1 b}}
\end{gathered}
$$

Shape functions and entrainment could also be added to the vorticity conservation model, and an assumption for the surface pressure change across the jump would not be required. However, as shown in $§ 3.3 .4$, the turbulent vorticity divergence term is significant for these high shear flows, so the balance between vorticity divergence of the mean flow and baroclinic production of vorticity would not hold.

These high-shear, two-layered flows are non-dimensionalized in the same way as the low-shear flows, described in $\S 2.2 .1$ and depicted in figure 3-3. Additionally, the entrainment fraction, $q_{\text {efrac }}=\frac{u_{1 b} d_{1 b}-u_{1 a} d_{1 a}}{u_{1 a} d_{1 a}}$ and shape parameters (3.2.4) are already non-dimensional. Non-dimensional solution spaces of the two-layer theory with entrainment and shape parameters are shown in figure 3-4 for various shear values. Results both with $\left(q_{\text {efrac }}=0.4\right)$ and without $\left(q_{\text {efrac }}=0\right)$ entrainment, 
and with $S_{1 b}^{2}=1.3^{2}$ and $S_{1 b}^{2}=1$, are shown for $r=0.1$ so that the effect of adding these parameters is apparent. The basic two-layer theory with $q_{\text {efrac }}=0$ and $S_{1 a}^{2}=S_{2 a}^{2}=S_{1 b}^{2}=S_{2 b}^{2}=1$ (solid black curves in the left column) gives solutions for low shear values $(s=0$ and $s=2)$. As shear increases to $s=5$, a theoretical solution space exists, although wave speeds at the interface are complex, indicating that the solution may be unstable and therefore physically inconsistent. This is also the range of shear values for which the solution space separates from the $R=1$ line, as noted in $\S 3.1$. The theoretical solution space disappears as shear increases to $s=8$ and above. Adding entrainment of $q_{\text {efrac }}=0.4$ to this theory (grey dashed line in left column) results in a theoretical solution space at all shear values. The shape of the solution space is also altered at low shear values: instead of a limited range of $U_{0}$ values for which jumps exist, adding entrainment allows small jumps with indefinite $U_{0}$. As shear increases to $s=5$, there are multiple solutions for a small range of $U_{0} \approx 5$. The upper branches predict jump heights similar to the solutions without entrainment, although real flows would likely produce jumps closer to the lower branch, where energy loss is maximized. For larger shear values $(s=8$ and $s=10$ ), solutions only exist if entrainment is allowed, and jumps can occur for any shear value. This suggests that entrainment is physically important in jumps with larger upstream shear.

The solution space with and without entrainment is shown with a shape parameter of $S_{1 b}^{2}=1.3^{2}$ in the right column of figure $3-4$. Using this shape parameter allows the theory to predict solutions at all shear values shown, even without including entrainment. However, based on previous work (Wilkinson and Wood, 1971; Regev and Hassid, 2010; Holland et al., 2002), adding entrainment is expected to be the most important physical difference between high and low shear jumps.

While the basic two-layer theory ceased to give solutions for shears of $s \gtrsim 7$, adding entrainment, shape parameters, or both results in a theoretical solutions space for much higher shear values. This implies that the governing physics changes as shear increases, and entrainment and continuous velocity profiles become necessary con- 
siderations. However, it should be noted that the value of the entrainment has been assumed, rather than calculated as part of the solution. The appropriate values of the shape parameters are also unknown, although values for several hyperbolic tangent profiles are given in figure 3-5. The effect of interface thickness and shear are shown. The values are typically close to one, but increase with increasing interface thickness and as $U_{0}-s \rightarrow 0$.

In the solution space with entrainment, three regions exist that categorize the behavior of the upper layer of the flow. These are depicted in figure 3-6 and indicated in the lower-left panel of figure 3-4. In region II, an exchange flow occurs, with the upper layer everywhere moving in the opposite direction of the lower layer. In region III, the flow is unidirectional, with the upper and lower layer moving in the downstream direction, both upstream and downstream of the jump. In region I, the flow in the upper layer is toward the jump, both upstream and downstream of the jump. This region is only possible because the lower layer entrains fluid from the upper layer, while regions II and III exist for flows without entrainment. As a result, region I spans a narrow range of $U_{0}$ values for typical values of entrainment. This work focuses on region III, so that fluid does not enter the computational domain through the outlet. 

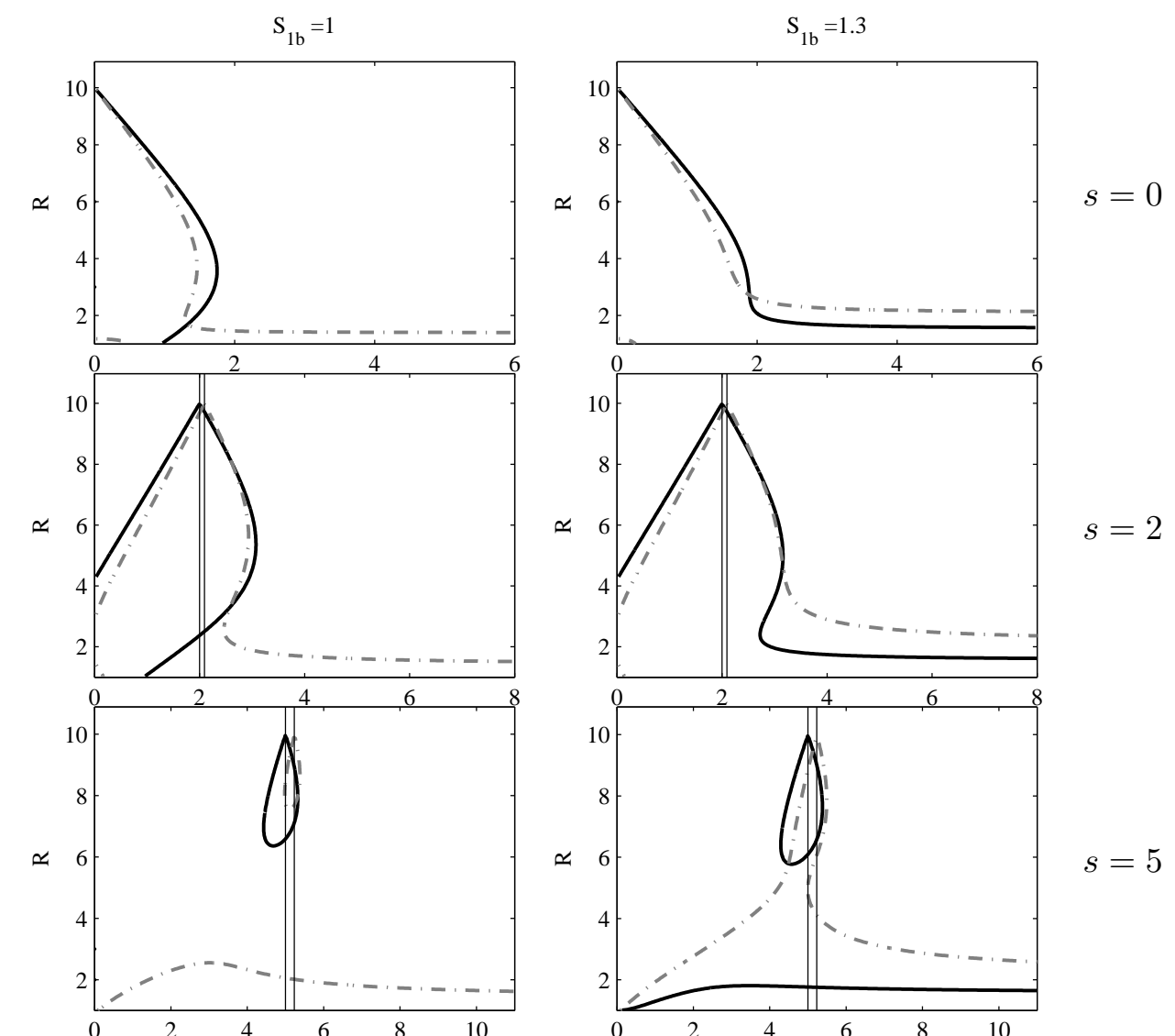

$s=5$
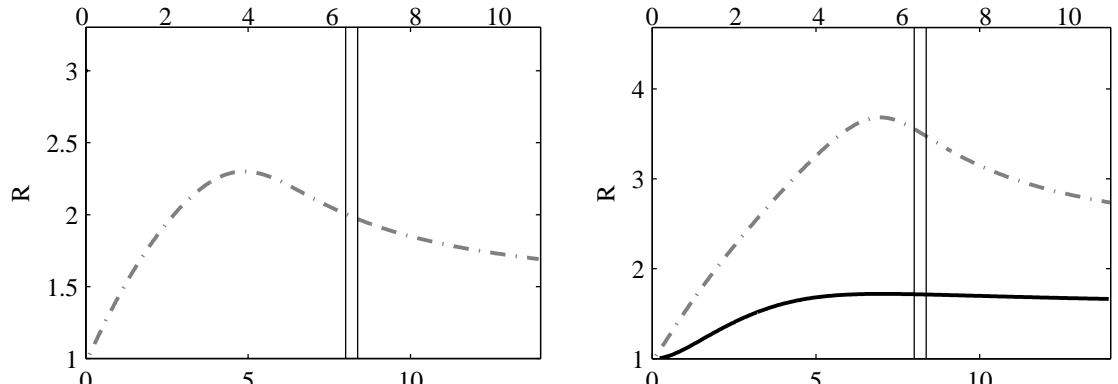

$s=8$
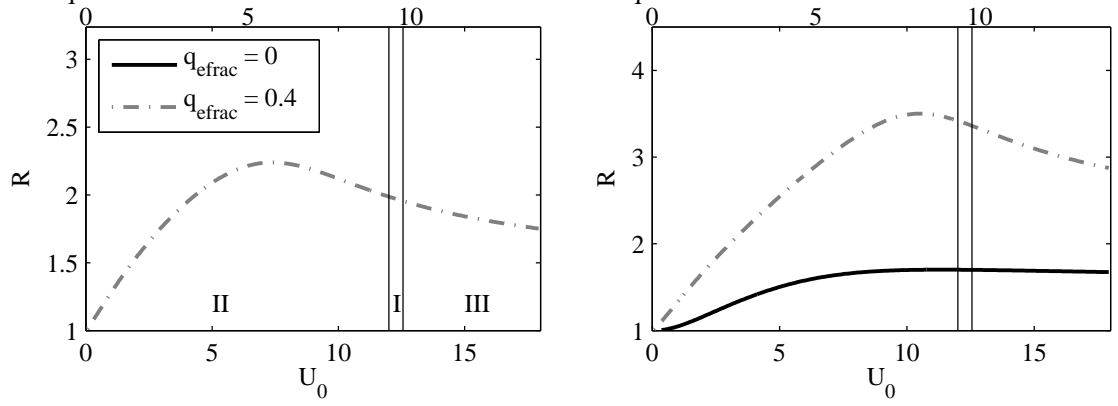

$s=12$

Figure 3-4: Theoretical results with $\left(q_{\text {efrac }}=0.4\right)$ and without $\left(q_{\text {efrac }}=0\right)$ entrainment. $S_{1 a}^{2}=S_{2 a}^{2}=S_{2 b}^{2}=1 . S_{1 b}^{2}$ is indicated on plot. $s=0$ (top), $s=2$ (second row), $s=5$ (middle), $s=8$ (fourth row), $s=12$ (bottom). 

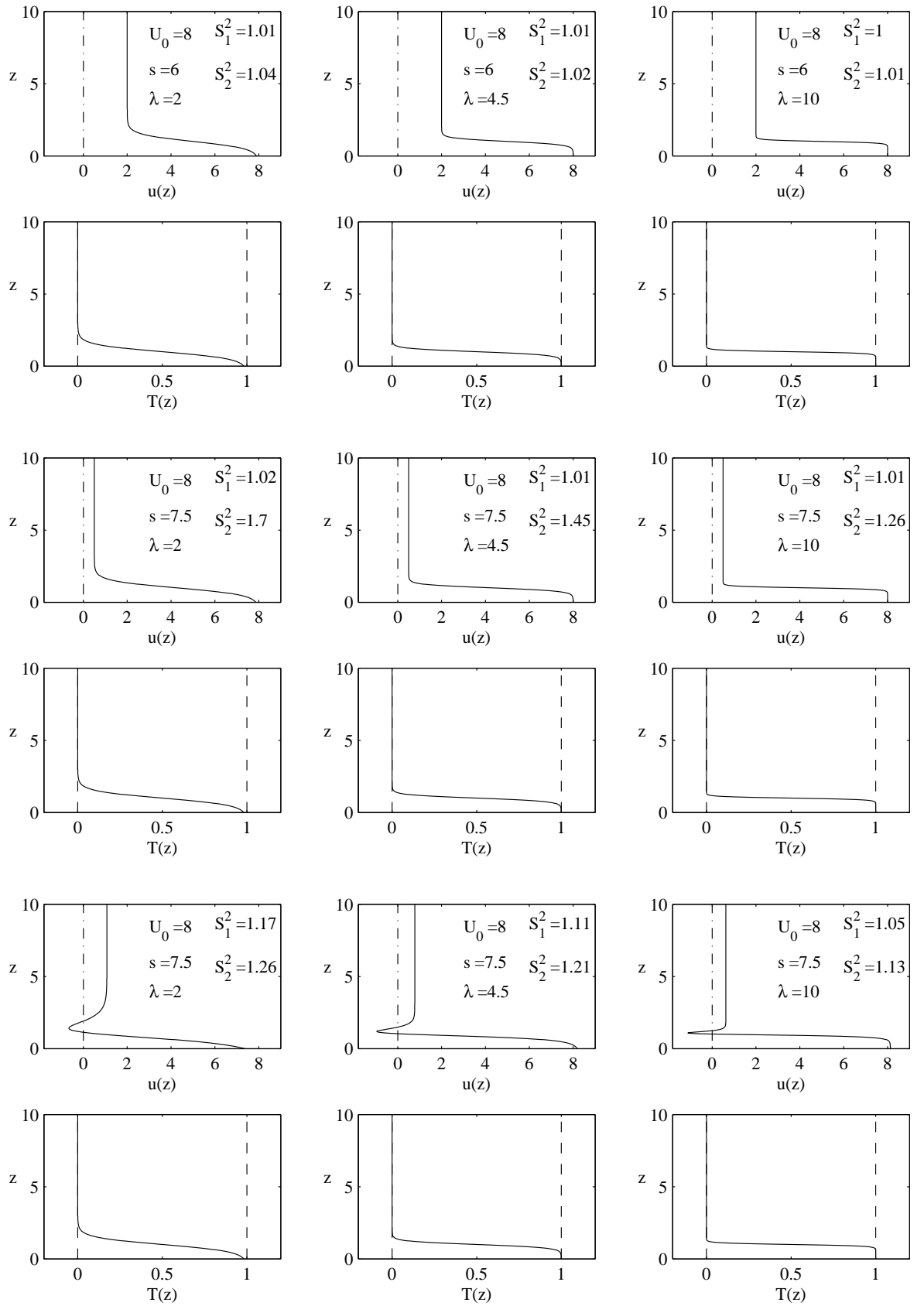

Figure 3-5: Shape parameters for sample profiles with varying shear, $s$, and interface thickness, $\frac{1}{\lambda}$. $\lambda$ increases (interface thickness decreases) to the right, and $s$ increases $\left(U_{0}-s\right.$ decreases) down. Bottom row shows effect of velocity minumum, compared to profiles with same volume flow rate and no minimum (middle row). 

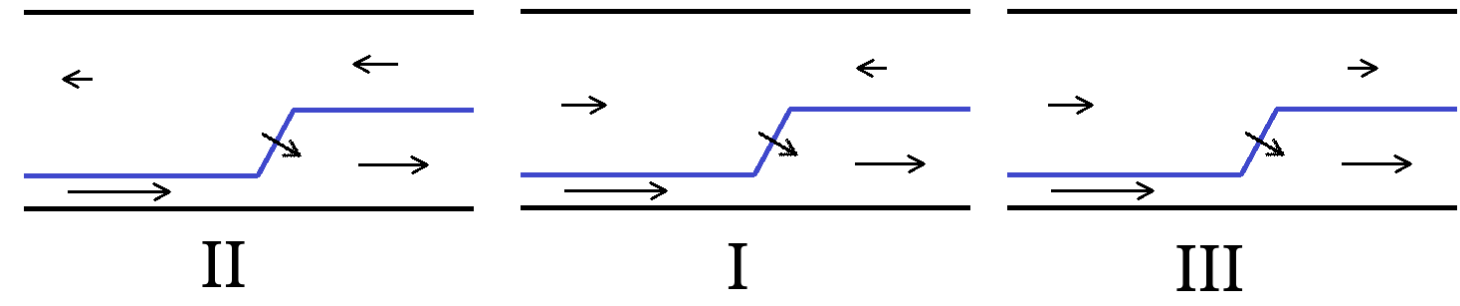

Figure 3-6: Behavior of upper layer for jumps with entrainment. 


\subsection{Numerical simulations}

As with the two-layer theories without entrainment and shape parameters described in chapter 2 , this extended theory $(3.2 .6,3.2 .7,3.2 .8,3.2 .9)$ neglects the dynamics within the jump region and simply matches upstream and downstream states using momentum flux, mass, and volume conservation. However, hydraulic jumps can involve important non-hydrostatic effects in the jump head region, as demonstrated in chapter 2 . Therefore, numerical simulations are conducted to understand qualitative characteristics of the jumps and to assess the validity of the two-layer theory. The simulations include continuous density and velocity profiles, although the upstream conditions are very close to two-layered, with a thin hyperbolic tangent interface.

The numerical simulations are conducted using the CFD software Gerris (Popinet, 2003), which is used in a non-hydrostatic, incompressible, Boussinesq mode. The model solves the incompressible Navier-Stokes equations with the Boussinesq approximation,

$$
\begin{aligned}
\frac{\partial \vec{u}}{\partial t}+\vec{u} \cdot \nabla \vec{u} & =-\frac{1}{\rho_{0}} \nabla p^{\prime}+\nu \nabla^{2} \vec{u}-g^{\prime} \hat{k} \\
\frac{\partial \rho^{\prime}}{\partial t}+\vec{u} \cdot \nabla \rho^{\prime} & =\kappa \nabla^{2} \rho^{\prime} \\
\nabla \cdot \vec{u} & =0 .
\end{aligned}
$$

The velocities are $\vec{u}=(u, v, w)$, where $u$ is along channel, $v$ is transverse (for $3 \mathrm{D}$ simulations only), and $w$ is in the vertical. The density deviation is $\rho^{\prime}, p^{\prime}$ is the pressure deviation from hydrostatic, $\nu$ is the kinematic viscosity, $\kappa$ is the density diffusivity,

$g^{\prime}=\frac{\rho-\rho_{0}}{\rho_{0}} g$ is the reduced gravity, and $\hat{k}$ is the unit vector in the vertical direction. The simulations described here have no explicit viscosity or diffusivity, although they experience mixing due to the numerical viscosity. The simulations are two-dimensional and have an isotropic grid with a resolution of 256 grid points in the vertical direction.

The numerical code uses the same basic advection algorithm as the IAMR model described in chapter 2 (Almgren et al., 1998), and as such uses the implicit large eddy simulation (ILES) method of handling turbulence, which is described in sec- 
tion 2.3 (Popinet, 2003). A different code is used in this chapter because the IAMR model does not allow topography, while the Gerris model does allow topography through the use of mixed cells, which are cut by the boundary (Popinet, 2003).

Unlike the simulations in chapter 2, where a jump was placed in the domain and allowed to develop, topography and a transient inlet condition are included in these simulations to trigger an internal jump. A different approach for developing a jump is taken here because the structure of the jump is unknown beforehand. Furthermore, the topography could affect the jump properties and may be used to make predictions about the jump, as discussed in §3.4.1. Without the topography and transient inlet condition, the upstream conditions could persist through the entire length of the domain. Topography also helps isolate the imperfect outlet boundary condition from the jump, reducing the effect of upstream-propagating disturbances on the jump. Finally, the topography could provide a constraint that helps determine the entrainment, which is the basis of the work by Wilkinson and Wood (1971); this approach is discussed further in $§ 3.4 .1$.

The simulations are initialized with a layer of denser fluid with a depth equal to the desired upstream lower-layer depth. The density transitions to a lower value through a hyperbolic tangent profile,

$$
T=\frac{1}{2}(1-\tanh (\lambda(z-1))), \quad T=\frac{\rho-\rho_{\min }}{\rho_{\max }-\rho_{\min }},
$$

with $\lambda=4$.5. This is shown in figure 3-7a. The velocity field is initially stagnant throughout the domain. The inlet conditions on the left side of the domain include a density profile matching the initial conditions within the domain. The inlet velocity ramps up smoothly from zero at the beginning of the simulation to the profile of the desired upstream conditions: 


$$
\begin{gathered}
u(z, t)=\left(U_{0}-s+s \frac{1}{2}(1-\tanh (\lambda(z-1)))\right) \\
\times\left(H(t-4 \pi)+H(4 \pi-t) \frac{1}{2}\left(1+\cos \left(\frac{t}{4}+\pi\right)\right)\right),
\end{gathered}
$$

where $H()$ is the Heaviside function. The outlet boundary conditions are $\frac{\partial u}{\partial x}=$ $\frac{\partial w}{\partial x}=\frac{\partial \rho}{\partial x}=0$ and $p=0$. This allows the fluid to fall out of the domain. If the barotropic flow is too slow, a bore will propagate into the domain along the upper boundary; however, the flows used in the simulations shown here are fast enough to avoid this problem. The topography also helps isolate the outlet boundary condition from the hydraulic jump upstream by transitioning the flow to supercritical just before the opening so that disturbances are unable to propagate upstream.

As the inlet velocity increases, a disturbance forms due to the pressure of the inlet flow against the stagnant fluid, as shown in figure 3-7b, which depicts the instantaneous tracer (or scaled density) field, $T=\frac{\rho-\rho_{\min }}{\rho_{\max }-\rho_{\min }}$ (previously referred to as $b$ in chapter 2), of a flow with $r=0.1, s=10.8, U_{0}=11.5, R=3.45$, and $q_{\text {efrac }}=0.45$ during the development of the jump. The disturbance propagates downstream and over the topography, eventually developing into a steady flow with a hydraulic jump in the region of the initial disturbance, as seen in figure 3-7c. Both the pressure gradient due to this initialization and the bottom topography contribute to the development of the jump.

The numerical results are non-dimensionalized and processed in the same way as the results from Chapter 2, described in $\S 2.3$, except that, due to the topography and the way the jump develops, the jump front is stationary, so the numerical results are naturally in the frame reference moving with the jump front. By identifying the interface using (2.3.8) and obtaining $U_{0}$ from the inlet conditions, the simulation results can be compared to the theory. The entrainment is calculated from the numerical simulation using 

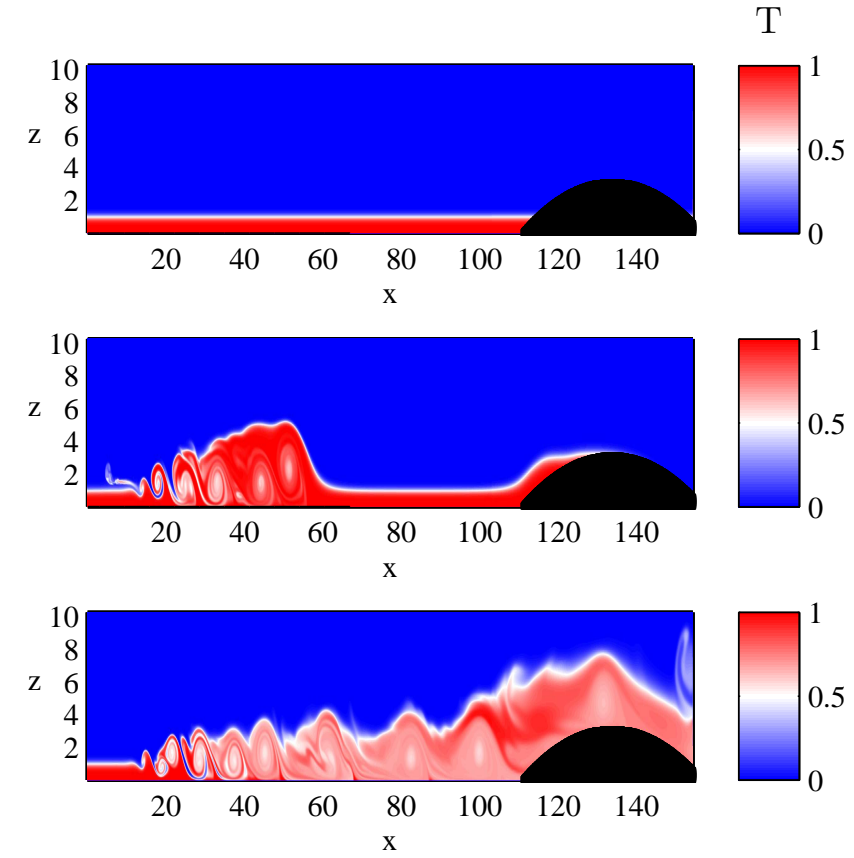

Figure 3-7: Instantaneous scaled density field. Initial conditions $(t=0)$, development $(t=10)$, and fully developed flow $(t=20)$ are shown for a jump with $r=0.1, s=10.8$, $U_{0}=11.5, R=3.45$, and $q_{\text {efrac }}=0.45$.

$$
q_{\text {efrac }}=\frac{\int_{x_{1}}^{x_{2}}\left(\int_{0}^{R} u(x, z)\right) d x}{x_{2}-x_{1}}-\int_{0}^{R} u(x=0, z) d z .
$$

where $R$ is defined in (2.2.13). In this definition, the entrainment is the difference between the downstream averaged volume flow rate of the lower layer and the upstream volume flow rate of the lower layer. The along channel points $x_{1}$ and $x_{2}$ define a dowsntream region after the jump, where the flow is no longer developing in the along channel direction.

\subsubsection{Simulation results}

Figure 3-8 shows simulation results for an example jump with a large upstream shear of $s=10.8$ and $r=0.1, U_{0}=12.7, R=1.9$, and $q_{\text {efrac }}=0.31$. The shape functions calculated from the simulation results are $S_{1 a}^{2}=1.01^{2}, S_{2 a}^{2}=1.05^{2}, S_{1 b}^{2}=1.04^{2}$, and $S_{2 b}^{2}=1.05^{2}$. The time averaged tracer field, with the interface indicated, shows that a steady jump occurs, despite the shear being outside the range of the solution space 
for the basic two-layered theory. The phase speed of the slowest wave $\left(c_{-}\right.$of the first internal mode in the long-wave limit calculated from the Taylor-Goldstein equation, described in Appendix B) is positive throughout the domain, indicating that all waves move in the direction of the flow. The flow therefore undergoes a super- to super-critical transition. The upstream profiles are very close to two-layered, with a thin smooth interface, as defined at the inlet. Downstream, the density profile is still approximately two-layered, with a thicker interface. The velocity profile in the lower layer is approximately linear. The theoretical solution curve is shown in figure 3-8c for $q_{\text {efrac }}=0.31$, which is calculated from the simulation. All shape parameters are set to one when calculating the theoretical solution. The numerical simulation is compared to the solution curve, and falls near the theory. For this simulation, the theory appears to predict the simulation results reasonably well without the use of shape parameters, if the entrainment is known beforehand. Vertical profiles of $T, u$, $R i_{g}$, and the first baroclinic mode wave structure, both upstream and downstream of the jump, are shown in figure 3-9. The wave mode structures do not have any zero-crossings, indicating that they correspond to the first internal mode. The wave speeds associated with these modes are the fastest and slowest in the domain, and are used to categorize the criticality of the flow. The wave speeds are also outside the range of $u(z)$, suggesting that critical layers do not occur.

A different simulation with a lower volume flow rate is depicted in figure 3-10. Again, despite the large upstream shear, a jump occurs. In this case, the phase speed of the slowest internal wave indicates that the flow transitions from super- to sub-critical, which is related to the lower volume flow rate; in general, for $r=0.1$, when $U_{0}-s=u_{2 a} \lesssim 0.8$, the flow will exhibit a super- to sub-critical transition. In the flow shown in figure 3-10, the upstream profiles are again very close to twolayered, as set at the inlet. However, the downstream profiles, while similar to those in figure 3-8, exhibit an important difference: the downstream velocity profile has a velocity minimum, and in fact a flow reversal, at a height of $z \approx 4.5$, close the region of the largest density gradient. This reversal results in a velocity profile that is closer to three-layered than two-layered, as the theory assumes. It also deviates 

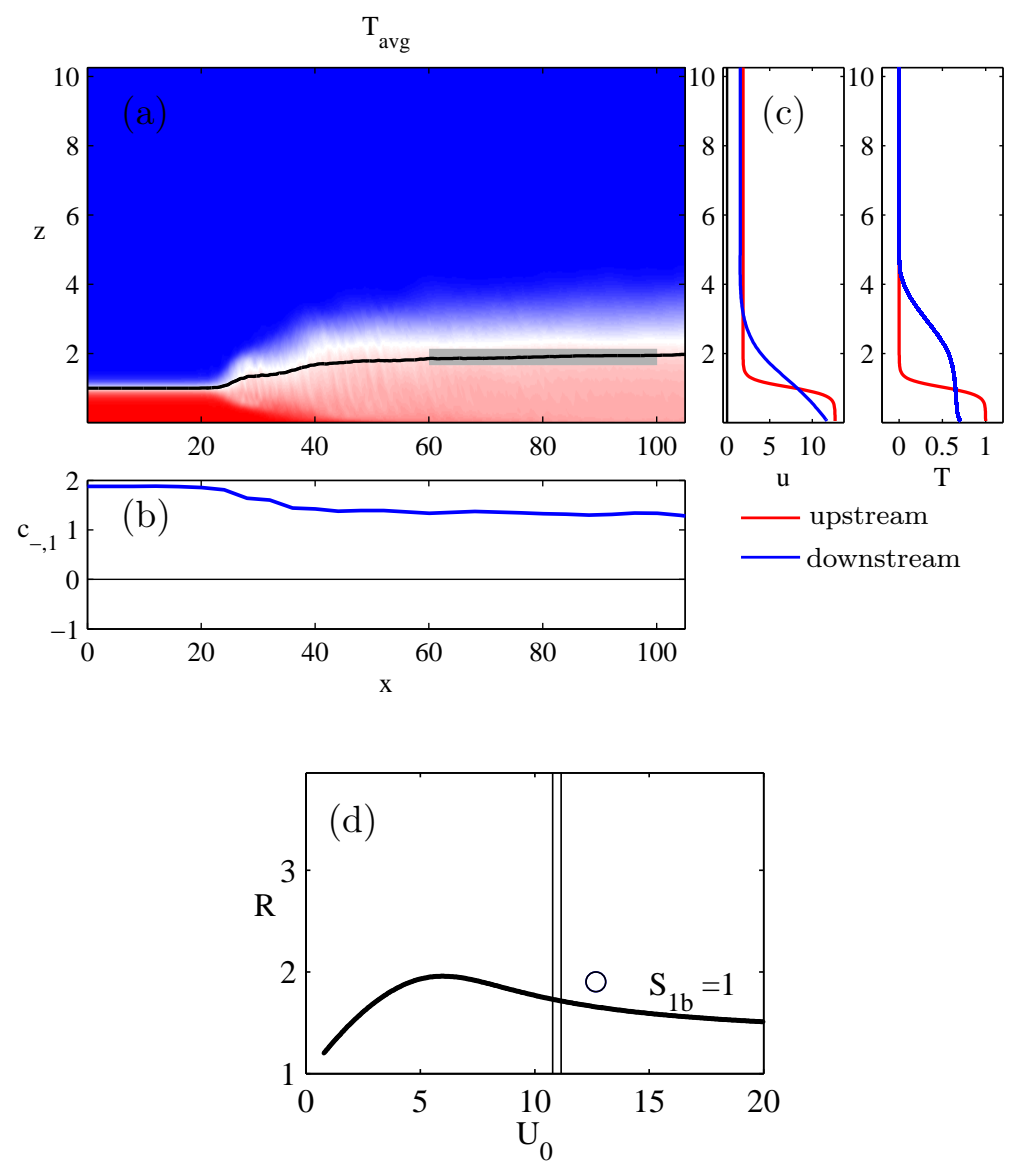

Figure 3-8: Super- to super- critical transition with high upstream shear. $r=0.1 ; s=$ $10.8 ; U_{0}=12.67 ; R=1.90 ; q_{\text {efrac }}=0.31, S_{1 a}^{2}=S_{2 a}^{2}=S_{1 b}^{2}=S_{2 b}^{2}=1.0$. (a) Timeaveraged tracer field of fully developed jump. (b) Phase speed of $c_{1-}$, the slowest moving wave, calculated using the continuous velocity and density profiles and the Taylor-Goldstein equation. (c) Upstream and downstream-averaged velocity and density profiles. (d) Simulation result compared to theory, with entrainment in the theory set equal to the value calculated from the simulation and $S_{1 a}^{2}=S_{2 a}^{2}=S_{2 b}^{2}=1$. 

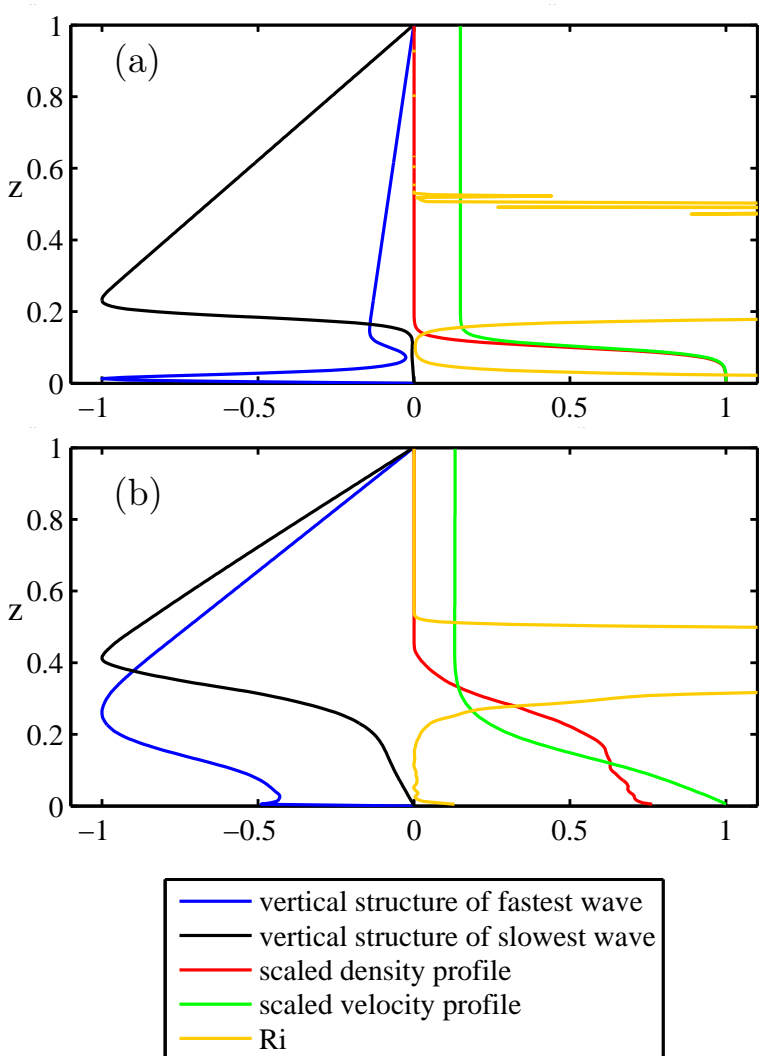

Figure 3-9: Density and velocity profiles, $R i_{g}$, and first-mode structure of super- to supercritical transition shown in figure 3-8. $r=0.1 ; s=10.8 ; U_{0}=12.67 ; R=1.90 ; q_{\text {efrac }}=$ $0.31, S_{1 a}^{2}=S_{2 a}^{2}=S_{1 b}^{2}=S_{2 b}^{2}=1.0$. (a) upstream at $x=0.08$ and (b) downstream at $x=58$. The vertical coordinate and the velocity are scaled to range from zero to one. 
from the unidirectional flow assumed, for example, by Thorpe (2010). Due to this deviation, shape parameters are used in the theory to obtain reasonable agreement with the simulation, as depicted in figure 3-10d. Figure 3-10d shows the solution curves, using a constant entrainment of $q_{\text {efrac }}=0.45$ calculated from the simulation, for three sets of shape parameters. For both curves, $S_{1 a}^{2}=S_{2 a}^{2}=S_{2 b}^{2}=1$. The lower curve uses $S_{1 b}^{2}=1$, the middle curve has $S_{1 b}^{2}=1.3^{2}$, which is closer to the value of the shape parameter calculated from the simulation, $S_{1 b}^{2}=1.6^{2}$. The upper curve uses $S_{1 b}^{2}=1.6^{2}$, which matches the value calculated from the simulation. The theory curve that uses $S_{1 b}^{2}=1.3^{2}$ agrees with the simulation result much better than the curve with $S_{1 b}^{2}=1$, indicating that the downstream vertical velocity profiles deviate from two-layered and must be accounted for in the theory. However, using the simulation value of $S_{1 b}^{2}=1.6^{2}$ in the theory does not agree as well with the simulation, indicating that the continuous profiles are not fully described by the shape parameters. Vertical profiles of $T, u, R i_{g}$, and the first baroclinic mode wave structure are shown for this super- to sub-critical transition in figure3-11.

A set of four simulations with the same upstream shear and total depth, but different values of $U_{0}$ is shown in figure 3-12. In figure 3-12a, the simulations are compared to a solution curve with $S_{1 a}^{2}=S_{2 a}^{2}=S_{1 b}^{2}=S_{2 b}^{2}=1$. The three simulations with larger $U_{0}$, which are all super- to super-critical transitions, fall near the theory. Figure $3-12 \mathrm{~b}$ shows the same set of simulations compared to the theoretical curve with $S_{1 a}^{2}=S_{2 a}^{2}=S_{2 b}^{2}=1$ and $S_{1 b}^{2}=1.3^{2}$. This theoretical curve matches the simulation with the smallest value of $U_{0}$, which is a super- to sub-critical transition. Both solution curves use a set value of $q_{e f r a c}=0.45$. Although the actual values of entrainment vary between the simulations $\left(q_{\text {efrac }}=0.36,0.35,0.41,0.45\right)$, all are reasonably close to this value. These results again show that for super- to sub-critical transitions, which occur for smaller values of $U_{0}-s(\lesssim 0.8)$, the continuous downstream velocity profile must be accounted for in the theory to obtain agreement with the simulation results. The values of the shape parameters calculated from the simulations and the effect on the theory of modifying the shape parameters is discussed in $§ 3.3 .2$. 

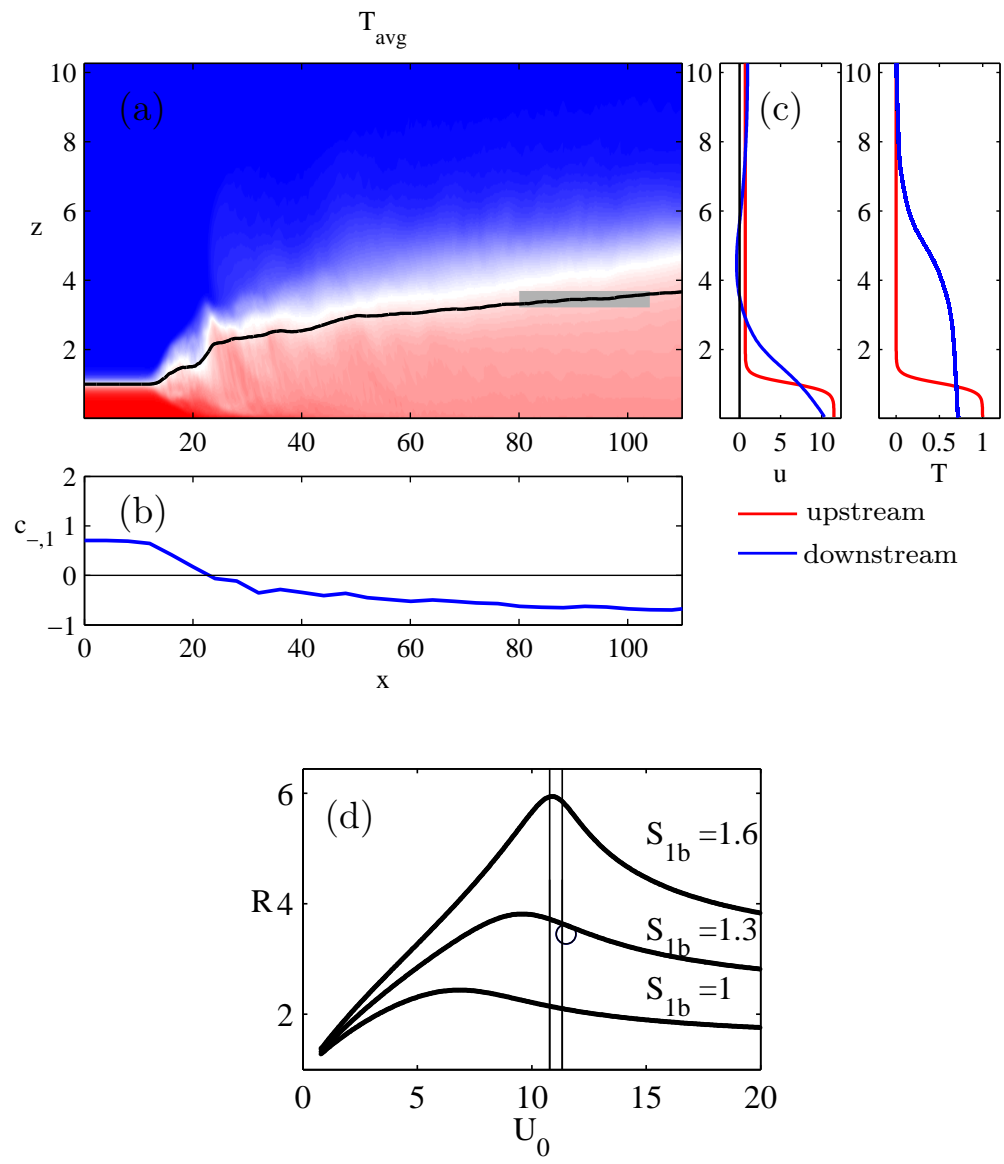

Figure 3-10: Super- to sub- critical transition with high upstream shear. $r=0.1 ; s=$ $10.8 ; U_{0}=11.5 ; R=3.45 ; q_{\text {efrac }}=0.45, S_{1 a}^{2}=1.0, S_{2 a}^{2}=1.3^{2}, S_{1 b}^{2}=1.3^{2}, S_{2 b}^{2}=1.6^{2}$. (a) Time-averaged tracer field of fully developed jump. (b) Phase speed of $c_{1-}$, the slowest moving wave, calculated using the continuous velocity and density profiles and the TaylorGoldstein equation. (c) Upstream and downstream-averaged velocity and density profiles. (d) Simulation result compared to theory, with entrainment in the theory set equal to the value calculated from the simulation and $S_{1 a}^{2}=S_{2 a}^{2}=S_{2 b}^{2}=1$. 

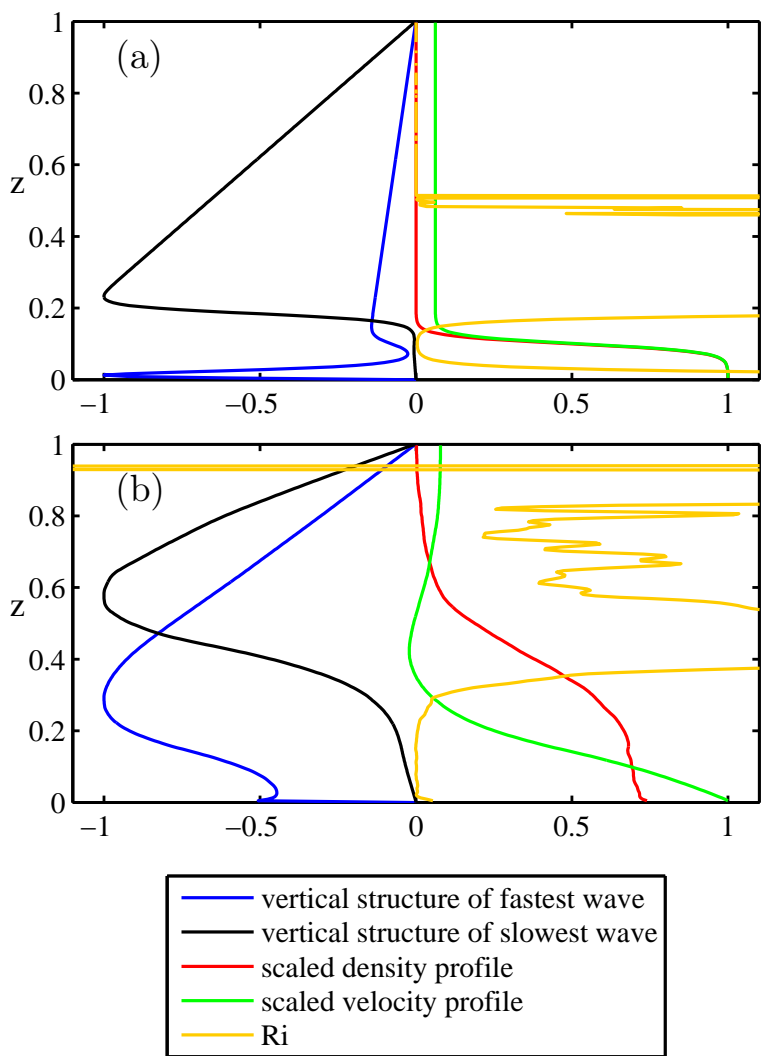

Figure 3-11: Density and velocity profiles, $R i_{g}$, and first-mode structure of super- to supercritical transition shown in figure 3-10. $r=0.1 ; s=10.8 ; U_{0}=11.5 ; R=3.45 ; q_{\text {efrac }}=$ $0.45, S_{1 a}^{2}=1.0, S_{2 a}^{2}=1.3^{2}, S_{1 b}^{2}=1.3^{2}, S_{2 b}^{2}=1.6^{2}$. (a) upstream at $x=0.08$ and (b) downstream at $x=58$. The vertical coordinate and the velocity are scaled to range from zero to one. 
Figure 3-12 also shows the downstream criticality of the two-layered flow with all shape parameters set to one, calculated using (2.2.14). Two-layered solutions in the red region of the solution space are super-critical downstream of the jump, whereas solutions in the blue region of the solutions space are sub-critical downstream of the jump. Only exchange flows, which occur in region I, exhibit sub-critical flow downstream of the jump, in the two-layered approximation. However, some simulations in region III, where both layers flow in the downstream direction, are actually sub-critical according to the internal long, linear wave speeds calculated from the Taylor-Goldstein equation. This demonstrates that the shape of the continuous velocity profile can have important qualitative effects of the flow that are not captured in the two-layer approximation. This is discussed further in §3.3.2.
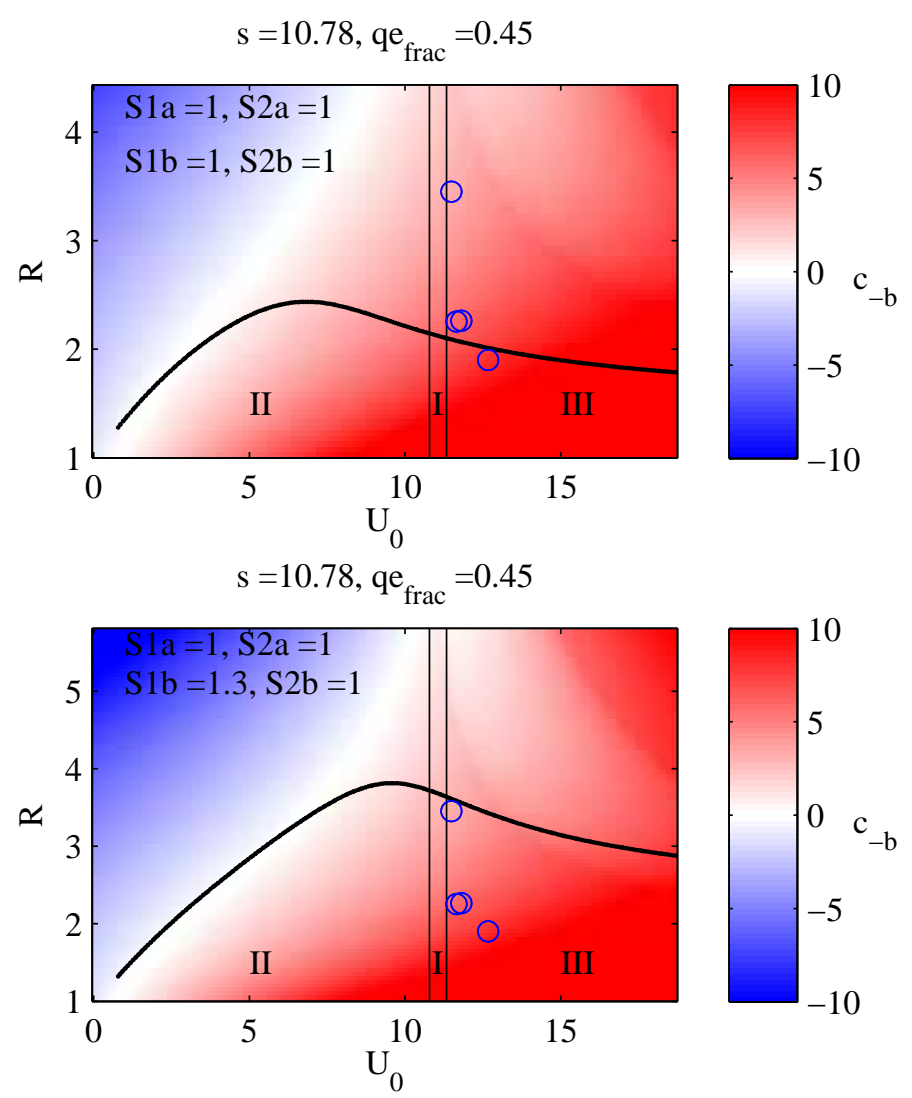

Figure 3-12: Numerical results compared to theory for two sets of shape funtions, with $r=0.1, s=10.8$, and $q_{\text {efrac }}=0.45$. Color indicates $c_{-}$of the two-layer flow. 


\subsubsection{Shape parameters and continuous profiles}

The numerical results produce flows whose velocity and density profiles are significantly different from the two-layered structure on which the theoretical model is based, particularly for super- to sub-critical transitions. Shape functions for the velocity are incorporated into the model to account for this deviation. However, the value of these shape parameters would generally not be known without measuring the flow, in which case the theory would not be needed to predict properties of the flow. Therefore, it is useful to understand the range of shape parameter values that are appropriate in the different flow regimes.

The values of the shape parameters from a set of simulations are shown in table 3.1. The simulations span a range of values of $s$ and $U_{0}$. The results show that the upstream shape parameters are all close to one, which is a result of how the upstream profiles are defined at the inlet. The downstream shape parameters are also approximately one $(1-1.1)$ for the super- to super-critical transitions. However, for the super- to sub-critical transitions, the downstream shape parameters deviate from one more significantly, indicating the significance of the continuous velocity profiles. In particular, the super- to sub-critical transitions exhibit a velocity minimum near the downstream interface of the jump, as seen in figure 3-10c.

\begin{tabular}{llllllll}
\hline Case & $s$ & $U_{0}$ & $S_{1 a}^{2}$ & $S_{2 a}^{2}$ & $S_{1 b}^{2}$ & $S_{2 b}^{2}$ & Downstream criticality \\
\hline RH-4x & 8.0 & 9.0 & 1.0 & $1.1^{2}$ & $1.1^{2}$ & 1.0 & super \\
RH-4c7b & 10.8 & 11.7 & 1.0 & $1.1^{2}$ & $1.1^{2}$ & $1.1^{2}$ & super \\
RH-4r2b & 20.0 & 21.1 & 1.0 & $1.2^{2}$ & $1.1^{2}$ & $1.1^{2}$ & super \\
RH-4c7d & 10.8 & 12.7 & 1.0 & 1.0 & 1.0 & 1.0 & super \\
\hline RH-4c7 & 10.8 & 11.5 & 1.0 & $1.3^{2}$ & $1.3^{2}$ & $1.6^{2}$ & sub \\
RH-4n & 4.3 & 5.2 & 1.0 & $1.1^{2}$ & $1.5^{2}$ & $1.2^{2}$ & sub \\
RH-4m & 5.1 & 5.9 & 1.0 & $1.1^{2}$ & $1.4^{2}$ & $1.3^{2}$ & sub \\
RH-4l2 & 6.7 & 7.1 & 1.0 & $1.1^{2}$ & $1.4^{2}$ & $1.3^{2}$ & sub \\
RH-4u & 8.0 & 8.9 & 1.0 & $1.2^{2}$ & $1.3^{2}$ & $1.4^{2}$ & sub \\
RH-4t & 9.3 & 10.1 & 1.0 & $1.2^{2}$ & $1.3^{2}$ & $1.5^{2}$ & sub \\
RH-4c5a & 10.8 & 11.5 & 1.0 & $1.3^{2}$ & $1.3^{2}$ & $1.8^{2}$ & sub \\
\hline
\end{tabular}

Table 3.1: Shape functions calculated from the simulation results for a set of simulations including super- to super- and super- to sub-critical jumps. $r=0.1$. 
Although there are four different shape parameters included in the theory, the value of $S_{1 b}^{2}$ is the most important. This is illustrated in figure 3-13, which shows theoretical solution curves with various combinations of shape parameters. The superto sub-critical transitions that require shape parameters different from one occur in the far left of region III. In this area, the figure shows that the theory is most sensitive to $S_{1 b}^{2}$. That is, holding $S_{1 b}^{2}$ constant and changing $S_{2 b}^{2}$ (black to green curve or red to blue curve) has little effect in the far left of region III. This is because, in this region, $u_{2 b}$ is very small, so the $S_{2 b}^{2} u_{2 b}^{2}$ term is small, and the precise value of $S_{2 b}^{2}$ is not very important. Therefore $S_{2 b}^{2}=1$ is used, and only $S_{1 b}^{2}$ is varied. This is the region of the solution space in which super- to sub-critical jumps occur. Although super- to super-critical jumps occur in a region where varying $S_{2 b}^{2}$ has a bigger impact on the solution curve, simulation results show that curves with shape parameters equal to one agree reasonably well with the simulations. This is because the density profiles are approximately two-layered and the velocity profiles do not exhibit the velocity minimum or flow reversal seen in the super- to sub-critical transitions. This work therefore only varies $S_{1 b}^{2}$, setting all other shape parameters to one. This also reduces the number of parameters that must be estimated to make predictions about the flow.

Figure 3-14 shows jump heights from a set of simulations compared to the jump height predicted by the theory, using the value of $q_{\text {efrac }}$ calculated from each simulation (simulation values range from $0.2 \lesssim q_{\text {efrac }} \lesssim 0.45$ ). In figure 3 -14a, all shape parameters are set to one. The group of simulations in the lower left of the figure all fall close to the $R_{\text {sim }}=R_{\text {theor }}$ line, indicating that the theory predictions match the simulations reasonably well. These are all super- to super-critical transitions, which have no flow reversal or velocity minimum in the vertical profile downstream of the jump, as seen in figure 3-8. The group of simulations with larger $R$ values, however, are grouped away from this line. In figure 3-14b, a shape parameter of $S_{1 b}^{2}=1.4^{2}$ is used in the theory for this group of simulations. This brings the predicted jump height much closer to the jump heights calculated from the simulations. This group of simulations corresponds to the super- to sub-critical transitions, and all exhibit velocity minima (and often reversals) in the downstream flow. This demonstrates 


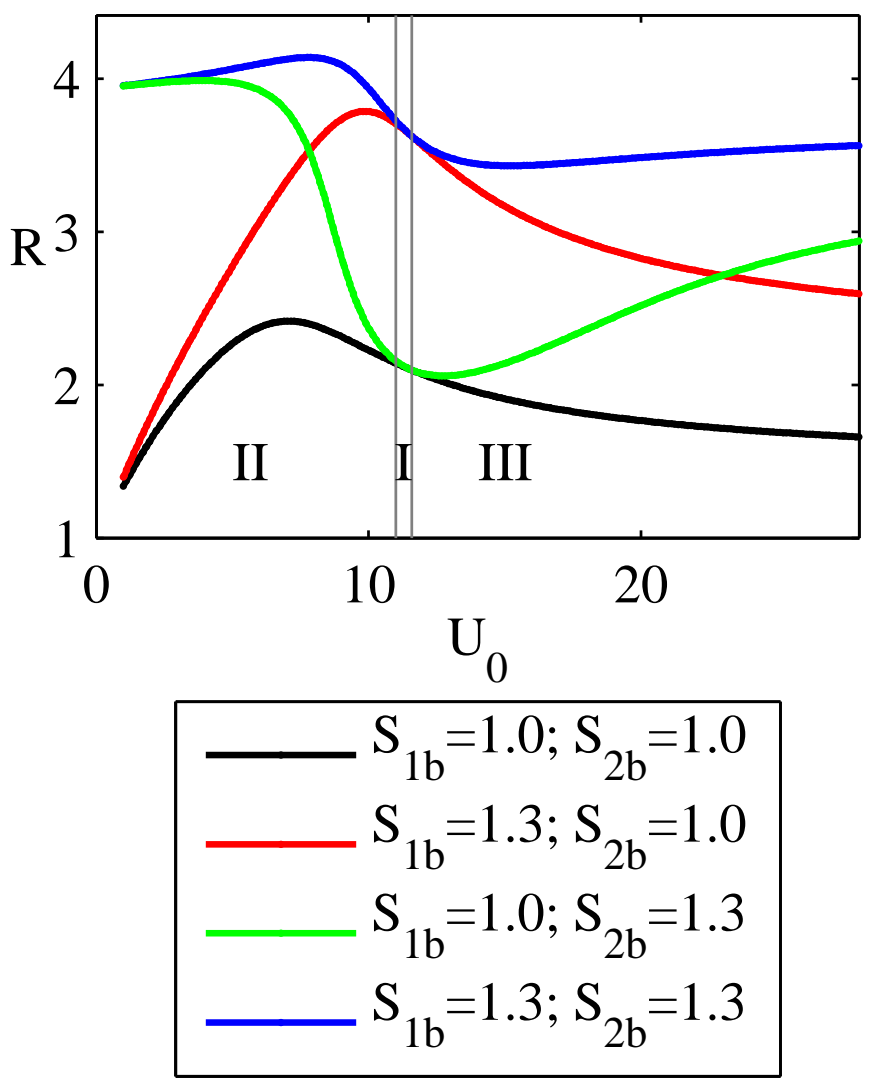

Figure 3-13: Theoretical solution curves with various combinations of shape parameters. $s=11, r=0.1, q_{\text {efrac }}=0.45$.

that using appropriate values of shape parameters to account for the velocity profiles seen in super- to sub-critical transitions is necessary to make predictions that are consistent with the numerical simulations. Furthermore, when the entrainment is found from the simulations, the theory is not highly sensitive to the value of the shape parameter. In figure 3-14, a constant value of $S_{1 b}^{2}=1.4^{2}$ gives reasonable results for all of the super- to sub-critical simulations.

As noted in §3.3.1, the shape of the velocity profile can make a flow that appears to be super-critical in the two-layer approximation, sub-critical according to the long wave speeds $(k=0)$ calculated from the Taylor-Goldstein equation. To explore the effect of the velocity minimum on $c_{ \pm}$, table 3.2 shows idealized continuous velocity and density profiles as well as the fastest and slowest phase speeds for each flow 

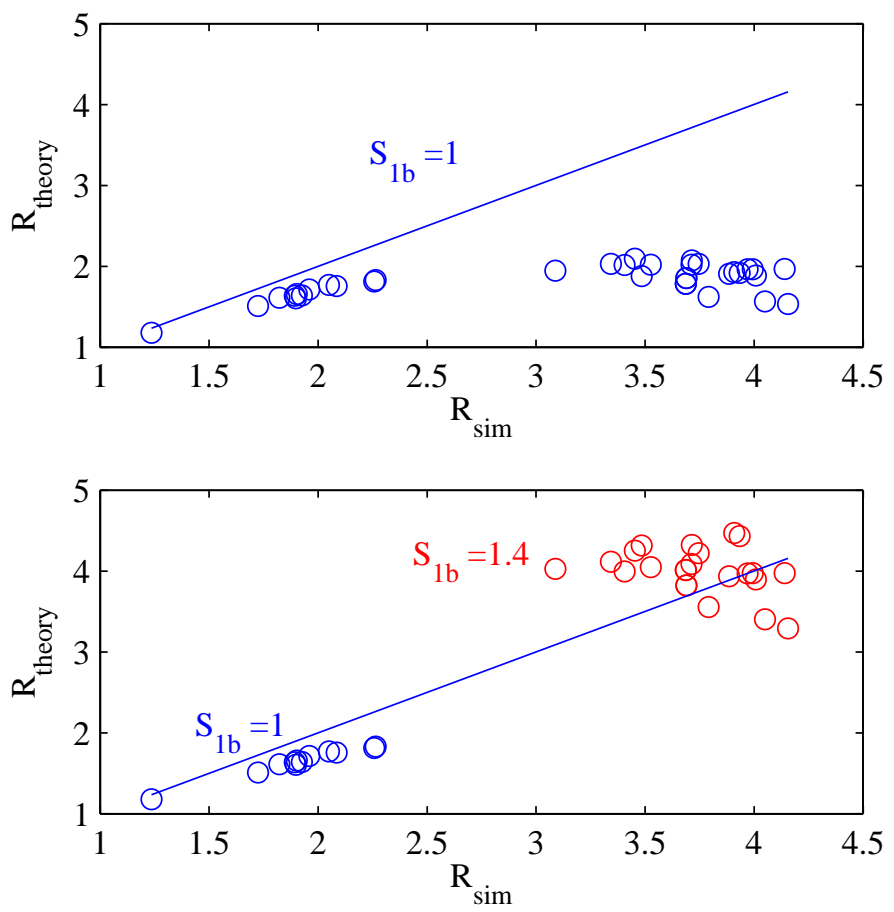

Figure 3-14: Numerical results compared to theory for all simulations using (a) $S_{1 a}^{2}=S_{2 a}^{2}=$ $S_{1 b}^{2}=S_{2 b}^{2}=1$ and (b) $S_{1 a}^{2}=S_{2 a}^{2}=S_{2 b}^{2}=1, S_{1 b}^{2}=1.4^{2} .0 .2 \lesssim q_{\text {efrac }} \lesssim 0.45$. The jump height predicted by the theory is plotted against the jump height calculated from the simulation for equivalent upstream conditions and entrainment.

(i.e. the lowest, long wave mode), calculated using the Taylor-Goldstein equation (Appendix B). The scaled density profile used for all cases is approximately twolayered, with a hyperbolic tangent interface, given by

$$
T=T_{1}+\Delta T \frac{\tanh \left(\lambda\left(z-z_{0}\right)-\tanh \left(-\lambda z_{0}\right)\right.}{\tanh \left(\lambda\left(1-z_{0}\right)\right)-\tanh \left(-\lambda z_{0}\right)}
$$

where

$$
z_{0}=0.2, T_{1}=1, \Delta T=-1, \lambda=45
$$

The velocity profiles are also hyperbolic tangents, with an added component to give the velocity minimum. The profiles are given by

$$
u(z)=V-\Delta V
$$


where

$$
V=u_{1}+\Delta u \frac{\tanh \left(\lambda\left(z-z_{0}\right)-\tanh \left(-\lambda z_{0}\right)\right.}{\tanh \left(\lambda\left(1-z_{0}\right)\right)-\tanh \left(-\lambda z_{0}\right)}
$$

and

$$
\Delta V=b+a\left(\frac{e^{\frac{-\left(z-z_{0}\right)^{2}}{f}}}{\int_{0}^{1} e^{\frac{-\left(z-z_{0}\right)^{2}}{f}} d z}-1\right) .
$$

The $\Delta V$ component gives the velocity minimum, and has the same volume flow rate for all profiles. The values of the constants are

$$
f=0.01, b=0.015, u_{1}=0.55, \Delta u=-0.48
$$

and $a=0,0.005,0.01,0.015$, for rows $1-4$, respectively.

Each net velocity profile $(u(z)=V-\Delta V)$ has the same volume flow rate $\left(\int_{0}^{1} u(z) d z\right)$. The flow depicted in the first row of table 3.2 has an approximately two-layered velocity profile without a velocity minimum. The flow described in the second row has a velocity minimum at the density interface, and each subsequent flow has a more prominant velocity minimum. The flow speeds show that when the velocity minimum is comparable to $c_{\min }$ for the flow without a velocity minimum, the flow becomes subcritical $\left(c_{\min }=0.035\right.$, and adding a velocity minimum of $a=0.015 \approx \frac{c_{\min }}{2}$ creates a sub-critical flow). However, a flow reversal is not required. Furthermore, the subcriticality in the hydraulic jump simulations is not due to the lower volume flow rate. It is due to the velocity minimum, which occurs in flows with a lower volume flow rate. This result shows that the detailed structure of the velocity profile is important, which is consistent with the use of shape parameters.

The shape parameters for each flow are also shown in table 3.2. A shape parameter larger than one occurs if there is a velocity profile that deviates from two-layered, including features such as a hyperbolic tangent transition between layers or a velocity minimum, and the deviation from one is larger if the layer velocity is smaller. The flow in the first row has a hyperbolic tangent transition but no velocity minimum, resulting in $S_{1}>1$. As the velocity minimum is increased, the upper layer velocity 


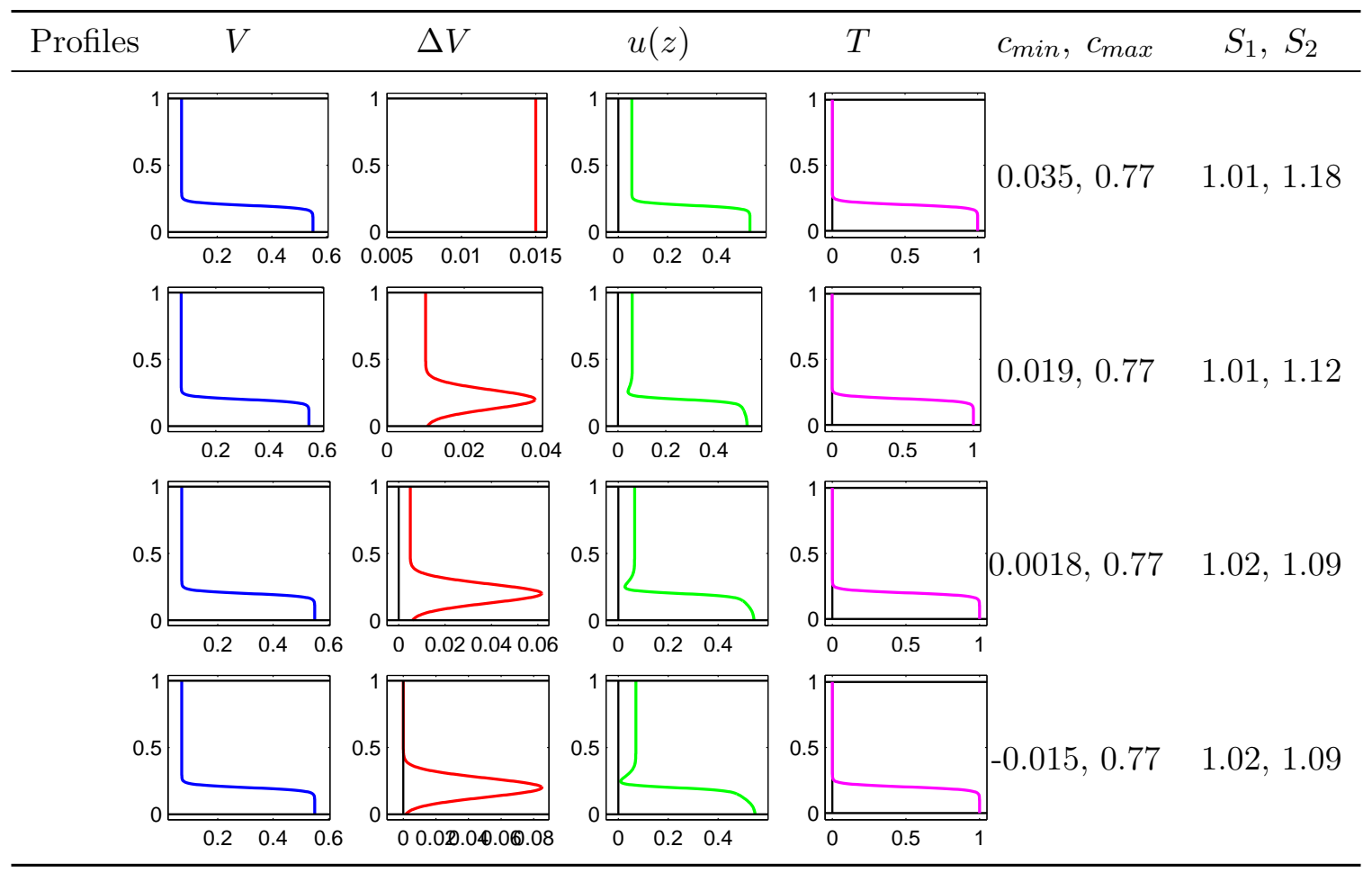

Table 3.2: Idealized density and velocity profiles and corresponding mode- 1 phase speeds. The background velocity $V$ and scaled density field $T$ is the same for all rows.

increases and the lower layer velocity decreases. Therefore, the lower layer shape parameter $S_{1}$ increases due to the increasing velocity minimum and the decreasing layer velocity. The upper layer shape parameter, $S_{2}$, is larger than one even without the velocity minimum, due to the hyperbolic transition. As the velocity minimum is increased, the upper layer velocity also increases, which allays the effect of both the hyperbolic tangent and the velocity minimum on $S_{2}$, causing a decrease. These results show that the shape parameters account for continuous profiles, but do not specify which features are present or prominant the features are. A shape parameter larger than one may occur due to a velocity minimum, a thicker interface, or another variation from strictly two-layered.

\subsubsection{Jump structure}

Wilkinson and Wood (1971) described jump structures in flows with deep, stagnant upper layers as having an entrainment region and a roller region, as sketched figure 3- 
15. In the entrainment region, fluid crosses the interface from the upper into the lower layer, mixing throughout the lower layer. In the roller region, fluid recirculates at the interface. Regev and Hassid (2010) also find these structures in their numerical simulations of hydraulic jumps in one-and-a-half-layer flows with high upstream shear (discussed further in §3.5). The detailed jump structure of two-layered flows with upstream shear varies depending on the type of jump, as shown in figure 3-16. For super- to sub-critical transitions, an entrainment region exists at the leading edge of the jump, shown at $x \approx 2$ in figure 3-16a. Downstream of the entrainment region are vortices that intersect the mean interface, seen from $x=3-6$ and centered on $y \approx 3$ in figure $3-16$ a. These might be equated to the entrainment and roller regions first described by Wilkinson and Wood (1971). In a super- to super-critical transition, such as in figure 3-16b, an entrainment region appears at the head of the jump, although the roller region (or downstream vortices) is absent. Intermediate, or double, jumps also exist, in which there is a super- to super-critical transition followed by a super- to sub-critical transition. An example is shown in figure 3-16c, where the super- to super-critical transition occurs from $1.5 \lesssim x \lesssim 3$ and the super- to sub-critical transition occurs for $3 \lesssim x \lesssim 5$. The intermediate jump can be compared to the theory as either a super- to super-critical transition with shape parameters of approximately one, where the region between $x=3-4$ in figure $3-16 \mathrm{~b}$ is considered the downstream region, or a super- to sub-critical jump with larger shape parameters of $S_{1 b}^{2} \approx 1.4^{2}$, where $x=4.5-5.5$ is the downstream region. Which jump type occurs depends primarily on $u_{2 a}=U_{0}-s$, and less so on $h_{T}$, the topography height, as illustrated in figure 3-17. Flows with $u_{2 a}=U_{0}-s \lesssim 0.8$ tend to be generate super- to sub-critical jumps, whereas flows with larger $U_{0}-s$ produce super- to super-critical jumps. The intermediate jump type exists for $U_{0}-s \approx 0.8$, and larger topography makes a super- to sub-critical transition more likely. Intermediate jumps are plotted twice in figure 3-17, once as a super- to sub-critical transition and once as a superto super-critical transition. 


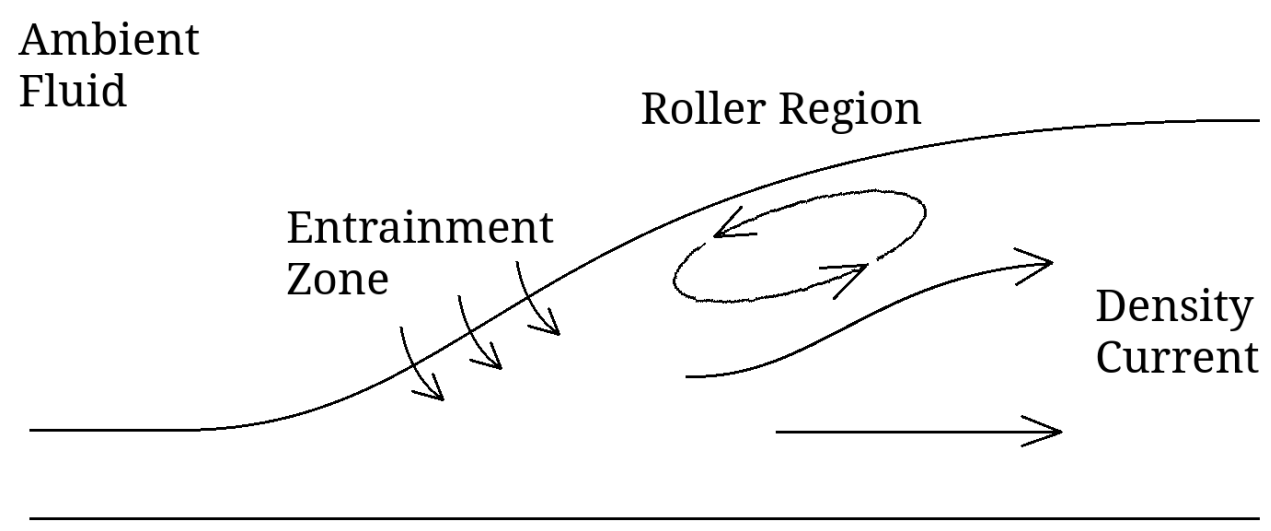

Figure 3-15: Flow structure described by Wilkinson and Wood (1971) for one-and-a-halflayer flows. 

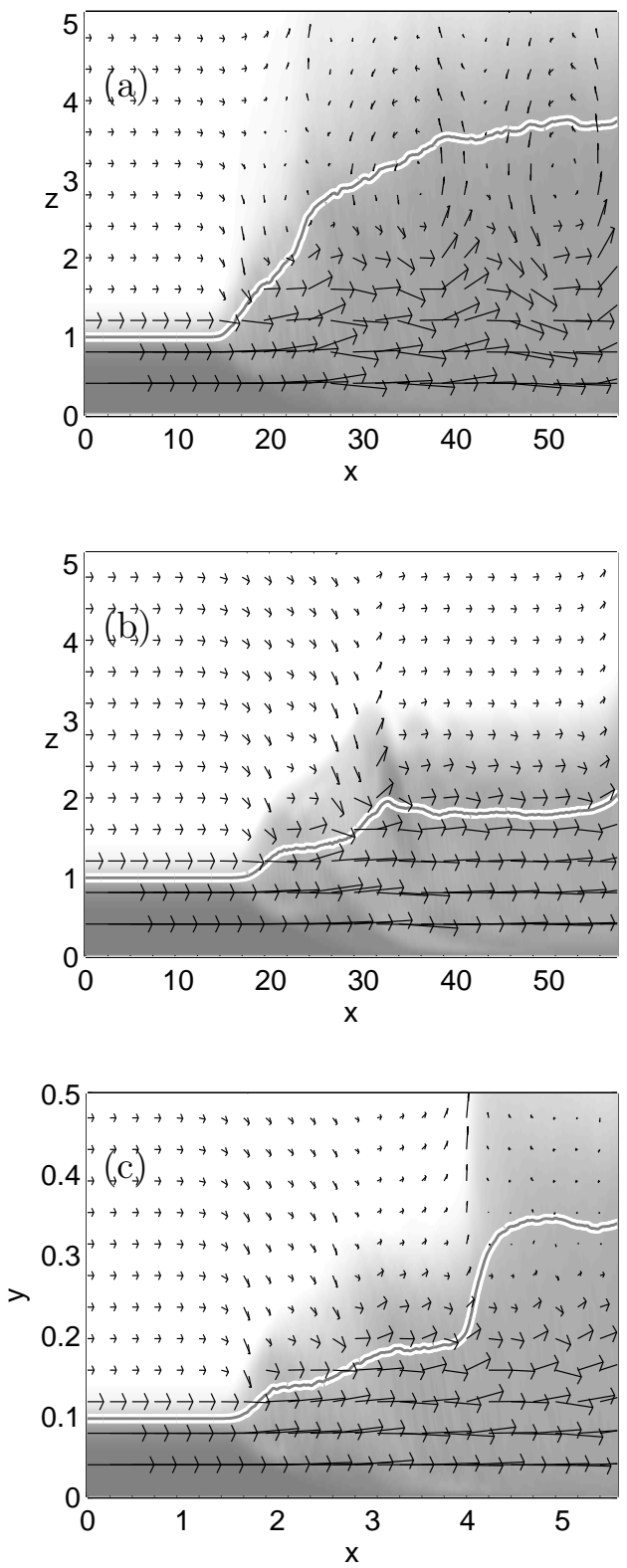

Figure 3-16: Jump structures of (a) a super- to sub-critical jump with $U_{0}=8.75$, (b) a super- to super-critical jump with $U_{0}=9.025$, and (c) a jump with a super- to super-critical transition and a subsequent super- to sub-critical transition with $U_{0}=8.85$. In all cases, $s=8$ and $r=0.1$. 


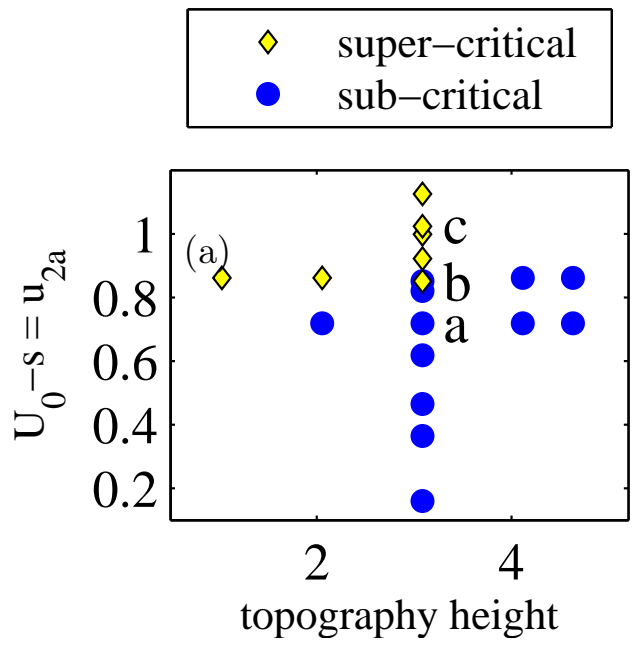

Figure 3-17: Regime diagram of jump structures. Double jumps are included as both superto super- and super- to sub critical transitions. 


\subsubsection{Vorticity budget}

As shear increases, the leading order balance of the vorticity budget (2.3.9) changes from that found in $\S 2.3 .2$, in which the mean baroclinic production balanced the mean vorticity flux divergence. Due to the increasing upstream shear, turbulent advection of vorticity becomes a more significant component than the baroclinic production of vorticity. The dominant vorticity budget balance begins to shift for jumps with shear in the transitional range of values $(3 \lesssim s \lesssim 7)$ through which the theory solutions without entrainment are lost. Figure 3-18 shows that for these intermediate shear values $(s=5.2$ and $s=6.7)$, the baroclinic production and turbulent vorticity divergence terms are comparable, and as shear increases, the turbulent vorticity divergence term becomes larger than the baroclinic production term. This occurs through the same range of shear values as the increase in entrainment and decrease in mixing efficiency, as discussed in $\S 3.7$.

(a)

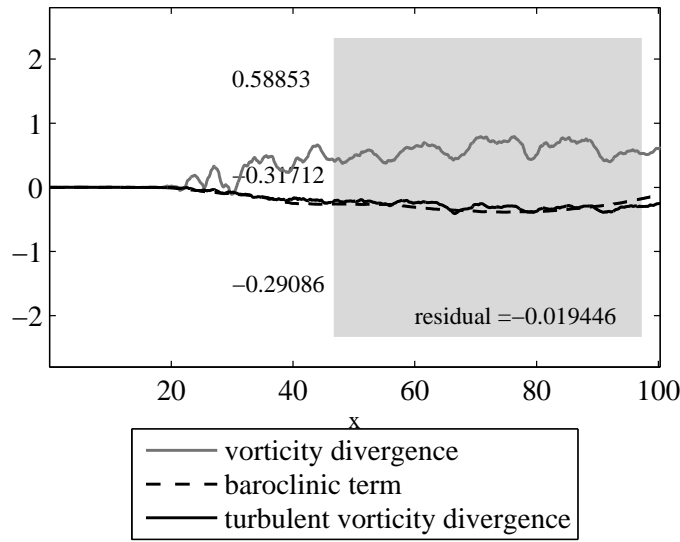

(b)

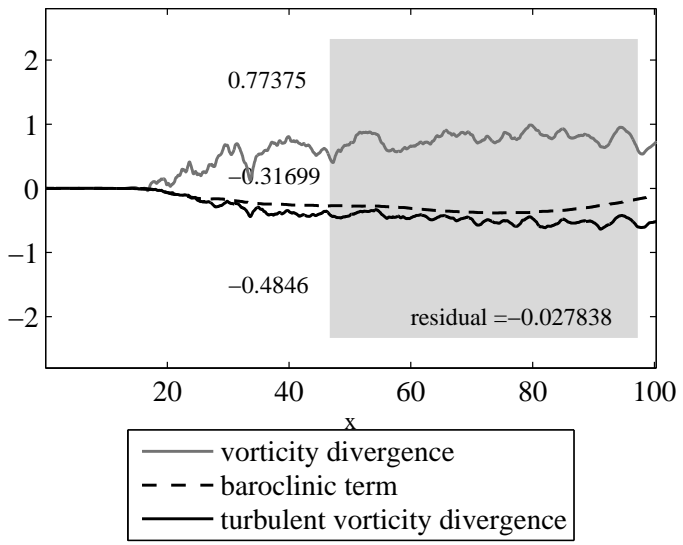

Figure 3-18: Cumulative integral of the vorticity budget terms for jumps with (a) $r=$ $0.1 ; s=5.2 ; U_{0}=5.9 ; R=3.7 ; q_{\text {efrac }}=0.34$ and (b) $r=0.1 ; s=6.7 ; U_{0}=7.1 ; R=$ $3.8 ; q_{\text {efrac }}=0.34$.

For jumps with large shear, the leading balance is between vorticity flux and turbulent vorticity flux, as shown for jumps with $s=10.8$ in figure $3-19$. The residual remains small compared to the other terms in the balance, indicating that the budget has been resolved well. The balance indicates that the vorticity conserving theory (Borden and Meiburg, 2013b) described in $\S 2.2$, which balanced baroclinic production 
of vorticty with mean advection of vorticity, is not applicable for these high shears because turbulent advection of vorticity becomes substantial as the upstream shear increases.

(a)

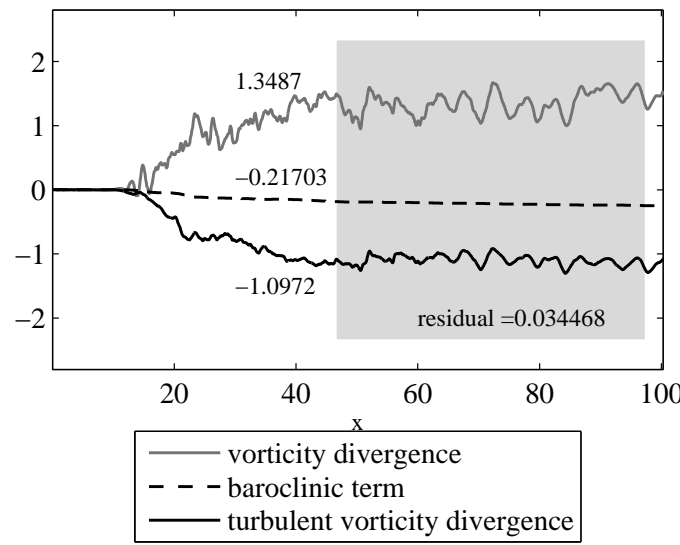

(b)

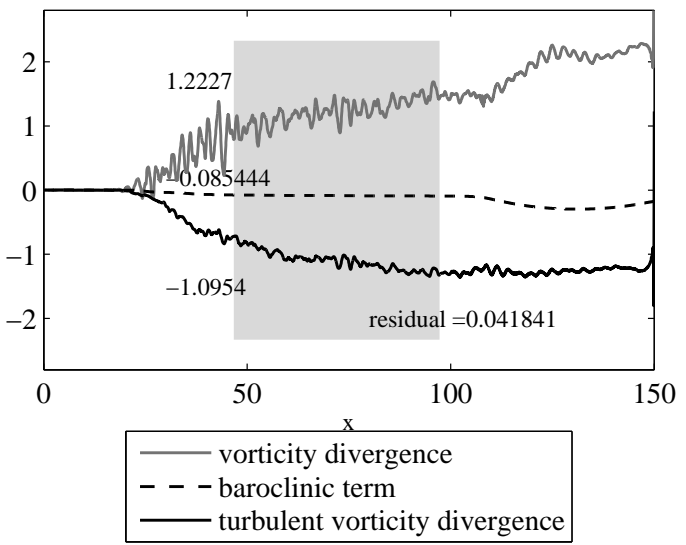

Figure 3-19: Cumulative integrals of the vorticity budget terms of (a) super- to super-critical $\left(r=0.1 ; s=10.8 ; U_{0}=12.67 ; R=1.90 ; q_{\text {efrac }}=0.31\right)$ and $(b)$ super- to sub-critical $\left(r=0.1 ; s=10.8 ; U_{0}=11.5 ; R=3.45 ; q_{\text {efrac }}=0.45\right)$ high shear jumps.

\subsection{Predicting entrainment}

The theory derived in $§ 3.2$ requires specifying the amount of fluid that is entrained into the lower layer. However, the entrainment should depend on the upstream and jump parameters and should be part of the solution. Approaches to calculating the amount of entrainment have been studied, for example by Wilkinson and Wood (1971) and Holland et al. (2002), whose appraoches are described here and in §3.4.1, and Thorpe (2010) and Milewski and Tabak (2015). Thorpe (2010) considers a continuously stratified flow under an infinitely deep, stagnant layer, and finds that entrainment is necessary for flows with $F r_{1} \gtrsim 7$. He finds a range of possible solutions with varying entrainment, and the actual solution depends on the upstream flow, the assumed structure of the downstream flow, and the specified entrainment. Solutions are found by conserving mass, volume, and momentum and by requiring that the upstream and downstream velocities are in the downstream direction, the density is uniform within 
a layer (but not necessarily across the jump), the flow is stably stratified, the density decreases across the jumps, fluid is entrained (not detrained), energy is lost across the jump, a specially defined Froude number is positive, and the flow is dynamically stable and stable to further jumps. Milewski and Tabak (2015) conserve energy instead of layer volume, allowing the energy that would be lost across the jump to mix fluid between layers. They include an adjustable parameter that determines the amount of mixing. The Holland et al. (2002) and Wilkinson and Wood (1971) ideas, which build on the theories explored in chapter 2, are extended to two-layer flows and compared to numerical simulations here, and a modified version of the Holland et al. (2002) approach is found to describe the entrainment well.

Holland et al. (2002) consider the lower layer, depth-integrated energy flux in a reduced gravity model, which, non-dimensionally is

$$
\left[\frac{d_{1} u_{1}^{3}}{2}+b d_{1}^{2} u_{1}+d_{1} u_{1} e\right]_{a}^{b}=0 .
$$

They conserve energy across the jump by assuming that the turbulent energy produced across the jump is converted to internal energy, $e$. They then assume that the internal energy is related to turbulent kinetic energy through

$$
e=d\left(\frac{1}{d_{1}} \int_{0}^{d_{1}} \frac{1}{2}<w^{2}>d z\right) ;<w^{2}>\leq b_{g} z .
$$

The upper limit on $<w^{2}>$ exists because the weight of the lower layer limits the production of turbulent kinetic energy. If more turbulent kinetic energy were produced, the energetic fluid would mix up higher into the water column, and the layer would be thicker. Therefore, the maximum internal energy is

$$
e=\frac{d}{4}\left(b_{g} h\right)
$$

where $d$ is a constant and $b_{g}$ is the scaled density,

$$
b_{g}=\frac{\rho-\rho_{2}}{\rho_{2}} .
$$


Holland et al. (2002) attribute $d$ to the number of dimensions of the flow, suggesting the value should be between 2 and 3 .

Noting that, through the mass and momentum conservation equations,

$$
\begin{gathered}
u_{1 b}=\frac{u_{1 a} d_{1 a}\left(1+q_{\text {efrac }}\right)}{d_{1 b}} \\
\rho_{1 b}=\frac{\rho_{1}+\rho_{2} q_{\text {efrac }}}{1+q_{\text {efrac }}}
\end{gathered}
$$

the entrainment, $q_{\text {efrac }}$, can be calculated using $(3.4 .1,3.4 .3,3.4 .4)$ in terms of the upstream parameters and the one unknown, $d_{1 b}$, or, non-dimensionally, $R$. The limit of $e$, given by (3.4.3) is used instead of finding a general expression for $q_{\text {efrac }}(e)$ because $e$ depends on $b_{g}(3.4 .3)$, which depends on $\rho_{1 b}$ (3.4.4), which depends on $q_{e f r a c}$ (3.4.6). If internal energy from turbulence is allowed both upstream and downstream of the jump, the entrainment is given by

$$
q_{\text {efrac }}=\left(\frac{d_{1 b}^{2}}{d_{1 a}^{2}}\left[1+\frac{2 g}{u_{1 a}^{2}}\left(1+\frac{d}{4}\right)\left(d_{1 a}-d_{1 b}\right)\left(\frac{\rho_{1}}{\rho_{2}}-1\right)\right]\right)^{\frac{1}{3}}-1
$$

So far, this is simply the Holland et al. (2002) approach applied to a two-layer flow. However, rather than calculating the entrainment from the jump height and a set value of $d$, the value of the constant $d$ can instead be determined (in non-dimensional parameters) using the jump height and entrainment from simulation results. This is given by

$$
d=\frac{2 U_{0}^{2}}{1-R}\left(\frac{1}{R^{2}}\left(q_{\text {efrac }}+1\right)^{3}-1\right)-4 .
$$

Similar expressions can be found if turbulent energy is only included downstream of the jump, which is reasonable for the flows with a laminar upstream that are studied here: 


$$
\begin{gathered}
q_{\text {efrac }}=\left[R^{2}\left(1+\frac{2}{U_{0}^{2}}\left[1-R\left(\frac{d}{4}+1\right)\right]\right)\right]^{\frac{1}{3}}-1 \\
d=\frac{4}{R}\left(1-\frac{U_{0}^{2}}{2 R^{2}}\left[\left(q_{\text {efrac }}+1\right)^{3}-R^{2}\right]\right)-4 .
\end{gathered}
$$

Figure 3-20 shows $d$ vs. $s$ for several simulations. When the internal energy is included both upstream and downstream, the super- to super-critical simulations diverge from the super- to sub-critical simulations as shear increases. However, when the turbulence related internal energy is included downstream but not upstream, all of the simulations collapse to a single curve, $d \sim s^{2}$. The simulations have a laminar upstream flow, so excluding a turbulence based internal energy term in the upstream is physically consistent with the simulations and is likely why all simulations collapse to a single curve when $e_{a}=0$. This indicates that the upstream shear is a controlling factor in the amount of entrainment. This fit is shown more clearly in figure 3-21 with the best-fit slope of $d \approx s^{2.05}$. This modified Holland approach for $q_{\text {efrac }}(3.4 .9)$ can be used in the theory $(3.2 .6,3.2 .73 .2 .83 .2 .9)$ from $\S 3.2$ to predict the entrainment as part of the solution. Solution curves, along which the entrainment varies, are compared to numerical simulations in figure 3-22.
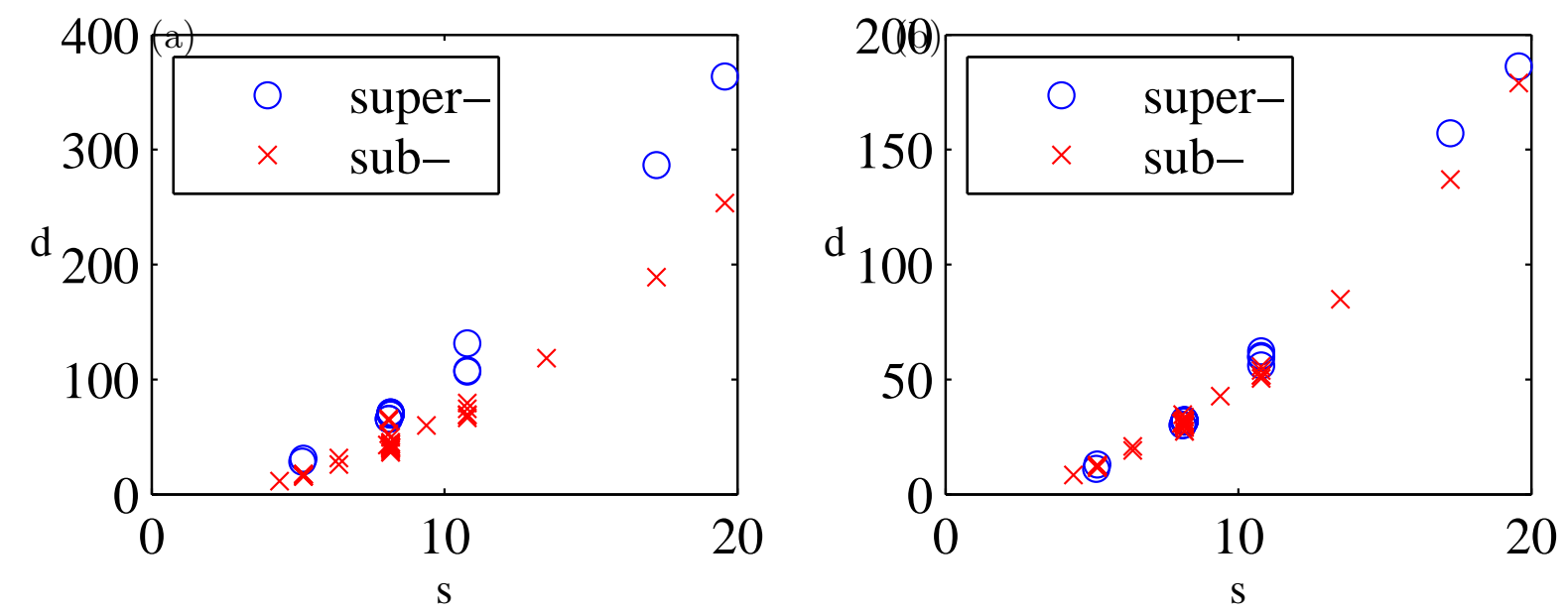

Figure 3-20: Value of constant $d$ versus shear $s$ (a) with turbulent energy upstream and (b) without turbulent energy upstream.

With this method, only the upstream conditions and the downstream lower layer 


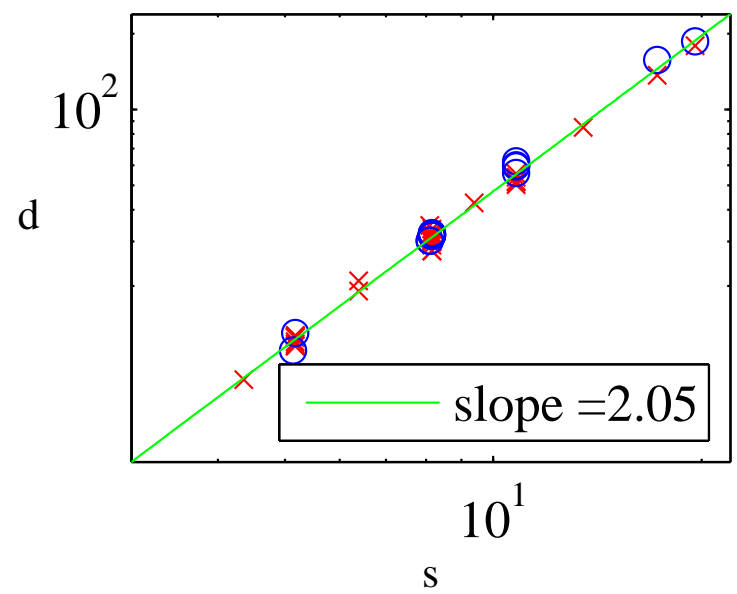

Figure 3-21: Value of constant $d$ versus shear $s$ with turbulent energy downstream.

shape parameter value, $S_{1 b}^{2}$, must be known to predict both the downstream jump height and the entrainment. Figure 3-22 shows that the entrainment predicted by the modified Holland theory with $d \sim s^{2}$ agrees well with the entrainment calculated from the numerical simulations. Although a curve fit was used in the development of the theory, the modified theory accounts for the physical processes (shear controlled entrainment and continuous velocity profiles) that are important. Figure 3-22 also illustrates that the jumps height is predicted well by the theory, if an appropriate shape parameter is used. However, the best shape parameter to match the simulation with the theory is not known a priori, and is not necessarily equal to the shape parameter calculated from the simulation. The shape parameters shown in figure 3-22 were chosen so that the curves pass through the simulation points.

The theoretical predictions for the modified theory that predicts entrainment $(3.2 .6,3.2 .7,3.2 .8,3.2 .9,3.4 .9)$ are compared to the full set of simulations in figure 323, which shows the theoretical jump height compared to the jump height calculated from the simulations (a), and the predicted entrainment compared to the calculated entrainment (b). For a given simulation, the theoretical jump height could match the simulation jump height exactly if the right shape parameter were used. However, this shape parameter would be different for each simulation, and it is also unknown. Therefore, $S_{1 b}^{2}=1.4^{2}$ is used for all super- to sub-critical simulations, and $S_{1 b}^{2}=1.08^{2}$ 


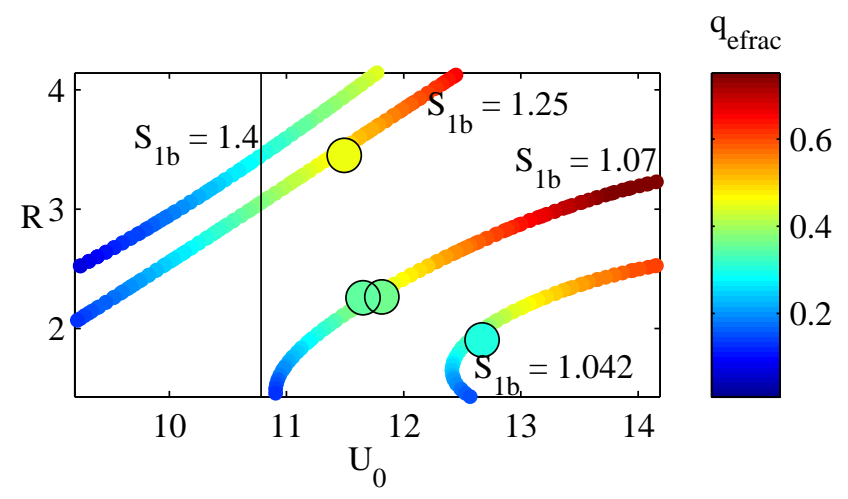

Figure 3-22: Simulation and theory results for modified Holland et al. (2002) model with turbulent energy downstream only. $s=10.8$ and $r=0.1$.

is used for all super- to super-critical simulations. As $S_{1 b}^{2} \rightarrow 1$, the theoretical solution curve of the modified Holland theory $(3.2 .6,3.2 .7,3.2 .8,3.2 .9,3.4 .9)$ shifts to higher values of $U_{0}$, as seen for $S_{1 b}^{2}=1.042^{2}$ in figure 3-22, and would not provide a solution for the range of $U_{0}$ used in the simulations, which is why $S_{1 b}^{2}=1.08^{2}$ is used. The criticality of the flow is diagnosed from the simulation results, and is not known a priori, although generally, simulations with $U_{0}-s=u_{2 a} \lesssim 0.8$ are super- to sub-critical transitions.

Figure 3-23a shows that, for super- to super-critical jumps, the modified theory that includes the prediction of entrainment does not predict the jump height as well as the theory in which the entrainment was calculated from the simulation and specified in the theoretical solution (seen in figure 3-14). This occurs for several reasons. First, using the actual value of entrainment calculated in the simulations is more accurate than predicting it using a model. Second, the modified theory that predicts entrainment is more sensitive to the shape parameters, particularly for superto super-critical jumps, so there is more scatter of the data when the same value of the shape parameter is used for a group of simulations. Finally, in the modified theory, the value of the entrainment changes along the curve (see figure 3-22), so if $U_{0}$ is slightly different, the solution curve predicts both a different $R$ value and a different $q_{\text {efrac }}$. The theory predictions of $R$ for super- to sub-critical transitions are comparable to the predictions from the theory in which the entrainment is specified 
(figure 3-14), which suggests that the modified Holland approach for predicting entrainment is performing well.

The entrainment predicted by the theory $(3.2 .6,3.2 .7,3.2 .8,3.2 .9,3.4 .9)$ is compared to the entrainment calculated from the simulations in figure 3-23b. Again, the theory works reasonably well for super- to sub-critical transitions, but has larger error for super- to super-critical transitions, which are more sensitive to the value of the shape parameter that is used. One point is predicted particularly poorly by the modified theory, for both jump height and entrainment. It is a super- to super-critical jump with a jump height of $R_{\text {sim }}=1.2$. It is predicted poorly because the shape parameter used by the theory, $S_{1 b}^{2}=1.08^{2}$, is much too large. A more appropriate value is $S_{1 b}^{2}=1.04^{2}$, which is the value calculated from the simulation results, or $S_{1 b}^{2}=1.03^{2}$, which provides an even better match for $R$ between the theory and the simulation. This shows that the theory, while consistent with the simulations, is not very useful for making predictions because the most appropriate shape parameter values are not known beforehand, and the theory is very sensitive to this parameter.

The fit $d(s) \sim s^{2}$ suggests that, as the upstream shear increases, the flow begins to behave as an expanding shear layer. As such, the jump region through which entrainment occurs is longer than the jump heads found in the low shear jumps described in chapter 2 . This is because the entrainment region extends in the along-channel direction until the mean gradient Richardson number at the interface is close to $R i_{g}=\frac{1}{4}$, and the flow becomes stable to Kelvin-Helmholtz instabilities. Figure 3-24 depicts the gradient Richardson number along the interface of both low and high shear simulations, with shear values of $s=1,1.5,5.1,10.8$. The flow with the lowest shear $(s=1$, figure 3-24a) is close to stable to Kelvin-Helmholtz instabilities at the interface upstream of the jump, and therefore undergoes a traditional hydraulic jump. When $s=1.5$ (figure 3-24b), the upstream flow has $R i_{g}<\frac{1}{4}$ at the interface. Still, the jump is abrupt with the jump head extending from $10 \lesssim x \lesssim 15$. $R i_{g}$ increases rapidly to $R i_{g}>\frac{1}{4}$ at the jump, and the transition does not behave as an expanding shear layer. Both of those flows also have negligible entrainment $\left(q_{\text {efrac }}<0.1\right)$. As the 
shear increases to $s=5.1$, the entrainment increases $\left(q_{\text {efrac }}<0.2\right)$ and the length of the jump head increases substantially, extending from $20 \lesssim x \lesssim 40$. When $s=10.6$, the jump is very similar to the $s=5.1$ case, with a long jump head, a transition to $R i_{g}>\frac{1}{4}$ near the end of the jump region, and a higher entrainment. These two higher shear jumps behave as expanding shear layers because the jump region through which entrainment occurs extends until the flow becomes stable to shear instabilities. However, all of these flows can be analyzed as hydraulic jumps if this shear-controlled entrainment is included across the jump. 

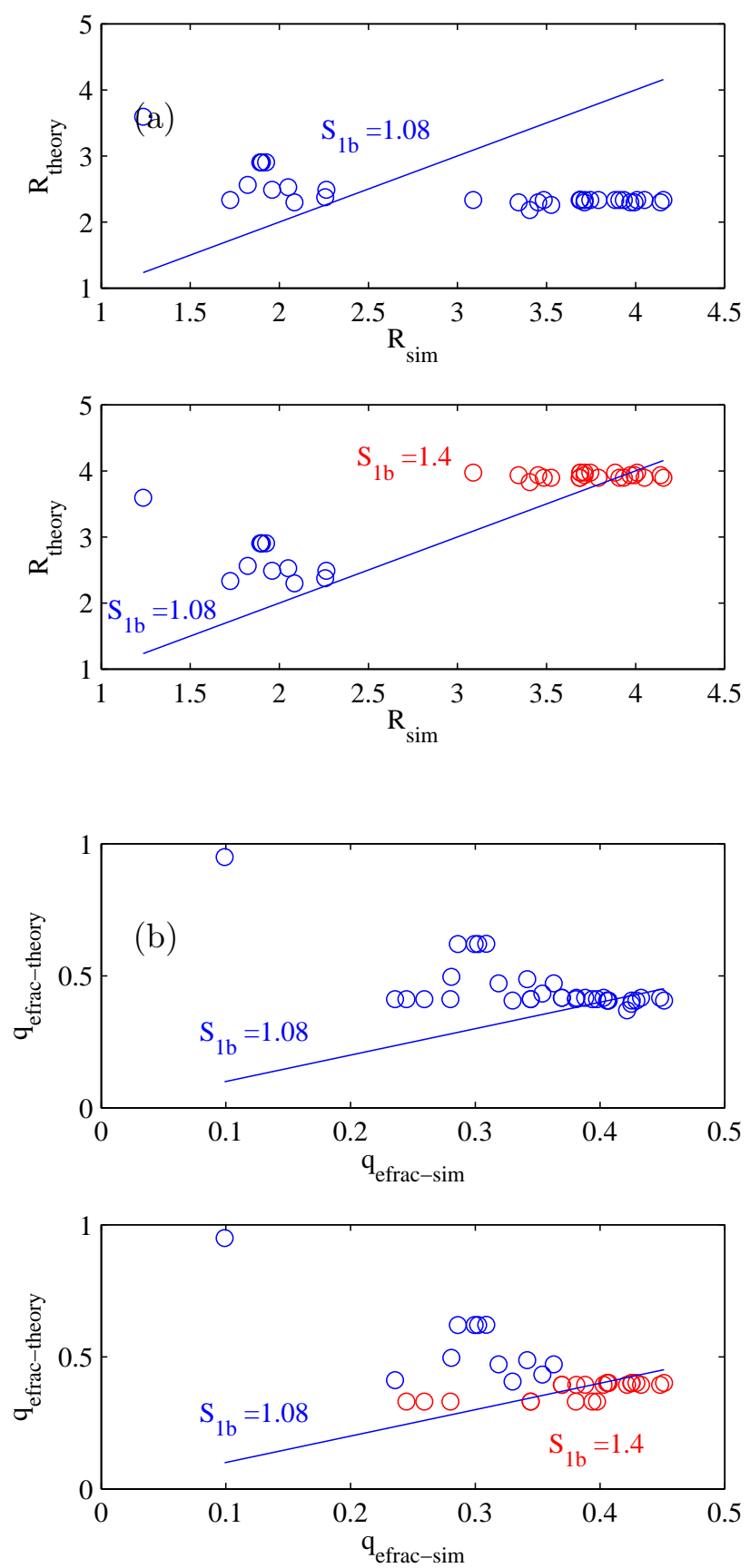

Figure 3-23: (a) $R_{\text {theor }}$ versus $R_{\text {sim }}$ and (b) $q_{\text {efrac,theor }}$ versus $q_{\text {efrac,sim }}$ using modified theory that predicts entrainment, with $S_{1 b}^{2}=1.08^{2}$ for all simulations in upper panel, and $S_{1 b}^{2}=1.08^{2}$ for super- to super-critical transitions (blue) and $S_{1 b}^{2}=1.4^{2}$ for super- to sub-critical (red) transitions in lower panel. 

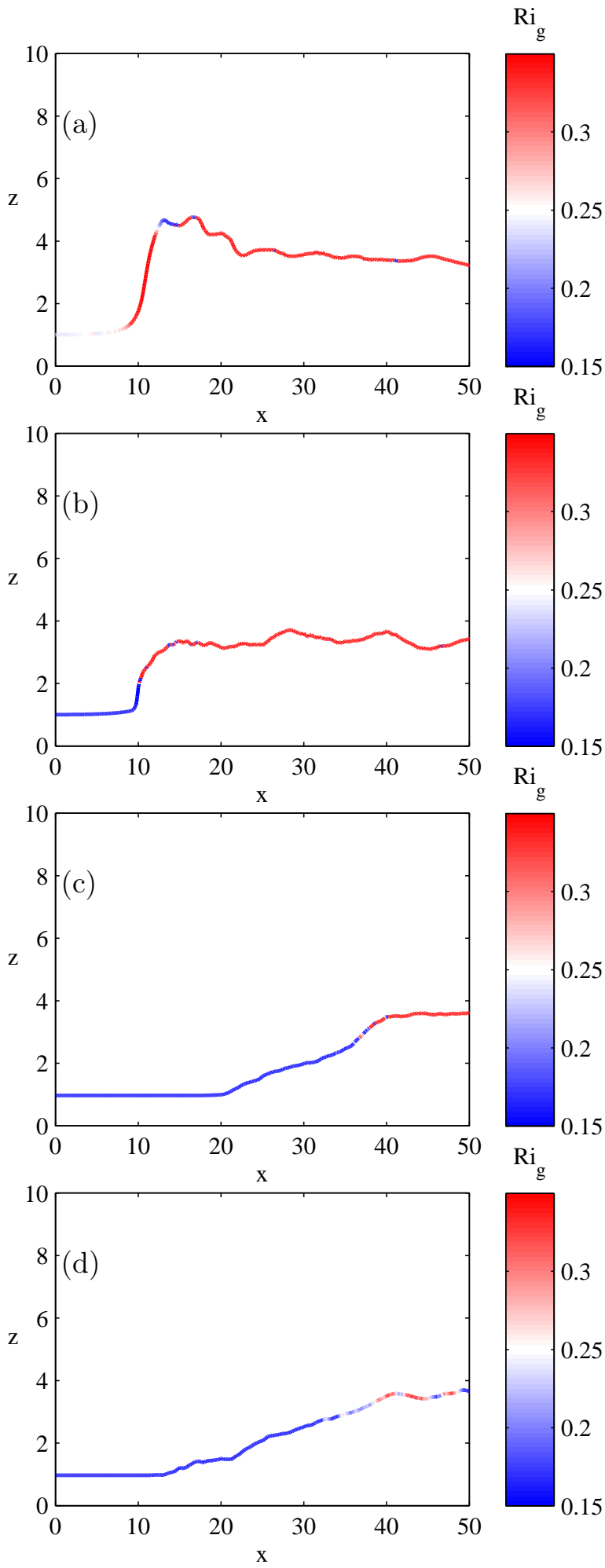

Figure 3-24: Gradient Richardson number for flows with (a) $s=1, U_{0}=1.8$, (b) $s=$ 1.5, $U_{0}=2.6$, (c) $s=5.1, U_{0}=5.8$, and (d) $s=10.6, U_{0}=11.3 . r=0.1$. Blue indicates regions of the flow that are unstable to Kelvin-Helmholtz instabilities, and red indicates regions of the flow that are stable, with white indicating neutral stability. 


\subsubsection{Wilkinson and Wood Approach}

Other approaches for predicting entrainment have been applied to the one-and-a-halflayer problem. For example, Wilkinson and Wood (1971) consider a one-and-a-halflayer flow over an obstacle, as depicted in figure 3-25a. They apply a shock joining approach between points a and b, and then conserve energy between points $\mathrm{b}$ and e, with the added constraint that the flow is critical over the sill. These requirements fully constrain the problem, and the entrainment is implicit in the solution. The application of this method to the two-layer problem with upstream shear, depicted in figure $3-25 \mathrm{~b}$, is considered here. The numerical simulations included in this chapter include downstream topography, so this approach for predicting entrainment applies to to the flows considered here.
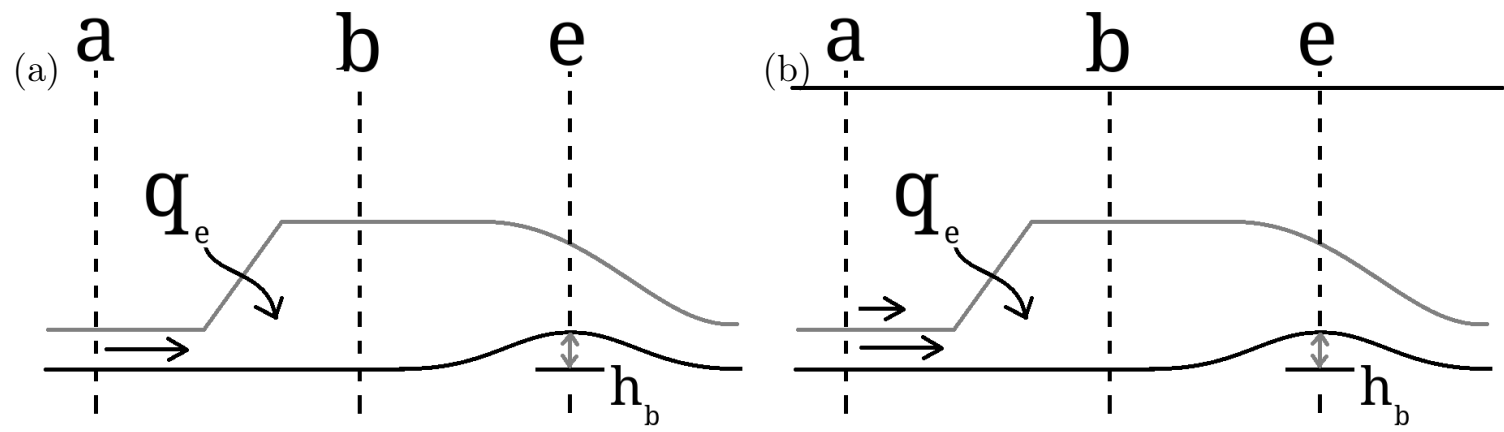

Figure 3-25: Flow setup of Wilkinson and Wood (1971) (a), and two-layer equivalent (b).

The Wilkinson and Wood (1971) approach can be applied to the two-layer flow in figure 3-25b using the shock joining methods described in $\S 3.2$, conserving energy flux in each layer between points $\mathrm{b}$ and e, and assuming that the flow is critical at the sill so that the sill height is a parameter in the solution. The equations for this approach are derived in Appendix A.

Wilkinson and Wood (1971) also include shape parameters in their derivation, which leads to shape parameters based on the cube of the velocity and on the density variation. These are new parameters, in addition to the shape parameters based on the velocity squared that come from the shock joining part of the solution, used in 
§3.2. This approach therefore depends on a larger number of unknown shape parameters. It further assumes that the flow is critical over the sill, thus incorporating the sill height into the solution. However, the flow is not necessarily critical over the sill in the continuous simualtions, as discussed below.

Predictions using this method for the two-layered flow are compared to numerical simulations in figure 3-26. Predictive curves are shown for three sill heights of $h_{b}=2,3$, and 4 . Numerical results are shown for simulations with sill heights of $h_{b}=1,2,3,4,5$, and 6 . The sill height does not affect the simulation results significantly, unless the sill is too short to force the flow to transition to sub-criticali downstream of the jump. If the sill is above this threshold height, the jump is superto sub-critical and the height of the sill does not significantly affect the jump because the flow is not critical at the sill, as noted in §3.3.3. If the sill were high enough, upstream adjustment would likely occur, although such simulations have not been conducted. For the theoretical solution curves with sill heights of $h_{b}=2$ and $h_{b}=3$, the super- to sub-critical simulation results are scattered near the solution curve, and the entrainment predicted by the theory matches the curve well. However, the theoretical curve for a sill height of $h_{b}=4$ does not extend to the $U_{0}$ value of the simulations, even though simulations produced jumps for this and larger sill heights. Furthermore, shape parameters were chosen to match the theoretical curves to the simulations as well as possible, rather than calculating appropriate values from the simulations. However, there are four shape parameters that deviate substantially from 1 , and their values are neither known beforehand nor consistent with the values calculated from the simulations. Therefore, this approach cannot be used predictively for analyzing hydraulic jumps and it is not consistent with the physics occuring within the jump.

Applying this method to the two-layer problem fails because the flow is not generally controlled at the sill, as the method assumes. The numerical simulations illustrate that the flow may be controlled at almost any point over the topography, or not at all, as described below. Furthermore, there are several important but unknown shape 
parameters to which the solution is sensitive, indicating the importance of the continuous profiles, which even the shape parameters fail to fully describe. The modified Holland approach is therefore applied in the remainder of this work.

The numerical simulations show several different qualitative types of flow control over the topography. An example of each type is shown in figure 3-28. In superto super-critical jumps with a strong barotropic flow, the flow remains super-critical over the topography, particularly if the topography is low enough. Similarly, superto sub-critical jumps followed by low topography exhibit sub-critical flow over the topography. However, if the topography is high enough, sub-critical flow after the jump may be controlled over the topography, either at or ahead of the sill. This is counterintuitive because two-layer flows are normally controlled at the sill, or downstream of the sill if the flow experiences bottom friction (Pratt and Whitehead, 2007). Regime diagrams showing various simulations and indicating the type of transition and the type of control that each exhibits are shown in figures 3-17 and 3-27. The control types depends on the upper layer velocity $U_{0}-s$ and less significantly on the sill height $h_{T}$. As $U_{0}-s$ increases from 0.2 to 0.4 , the flow transitions from sub-critical over the topography to critical at the sill crest. As $U_{0}-s \rightarrow 0.5$, the control point moves upstream to a position over the topography but ahead of the crest. The location of control varies with $U_{0}-s$, and when $U_{0}-s \gtrsim 0.9$, the flow remains super-critical over the entire topography. Examples of flows with different locations of control are shown in figure 3-28. The time-averaged scaled density field and $c_{-}$for the first two modes, calculated from the continuous density and velocity profiles using the Taylor-Goldstein equation (Appendix B), are shown for four flows, including a flow that remains sub-critical over the topography, a flow that is controlled at the sill crest, a flow that is controlled upstream of the sill crest, and a flow that remains super-critical over the topography. The various ways in which the flow can be controlled are one of the reasons why the Wilkinson and Wood (1971) approach, which assumes that the flow is controlled at the crest of the sill, fails when applied to two-layered flows. 

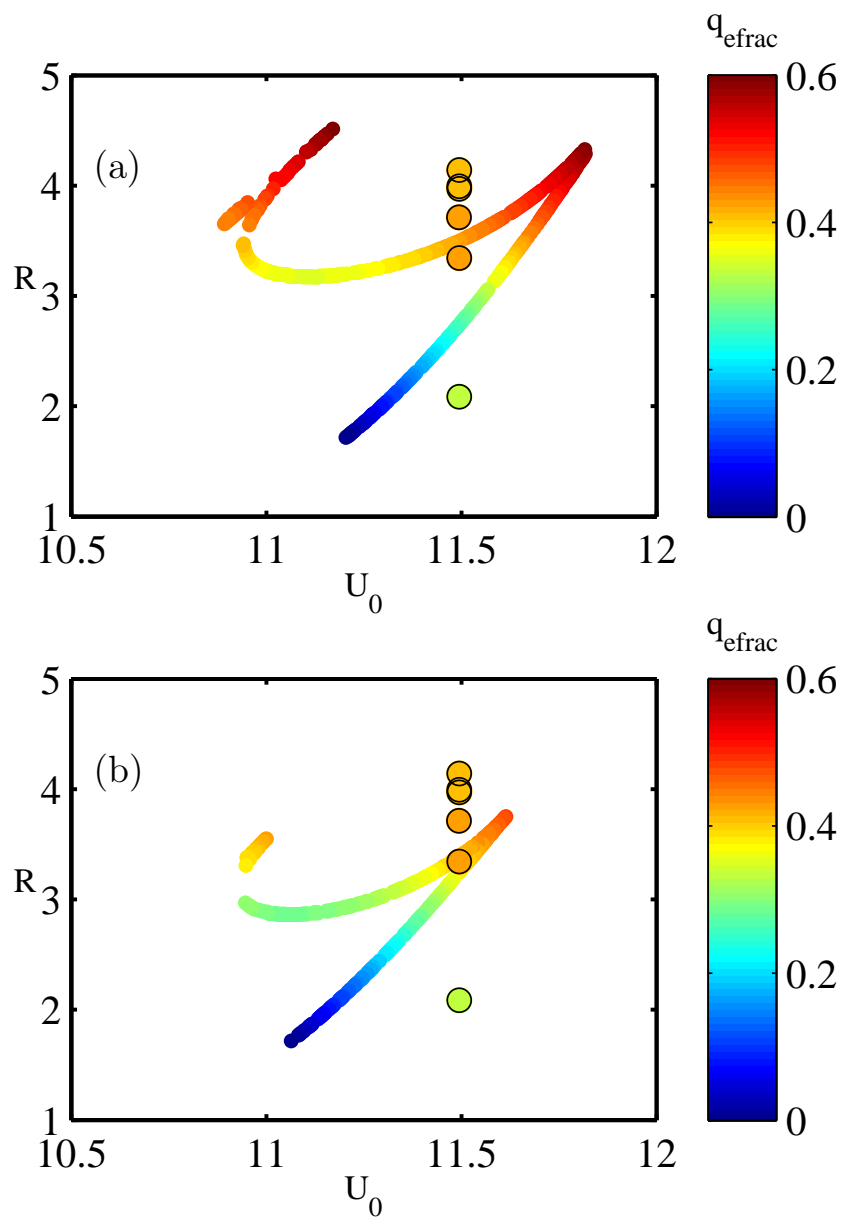

$\mathrm{q}_{\text {efrac }}$
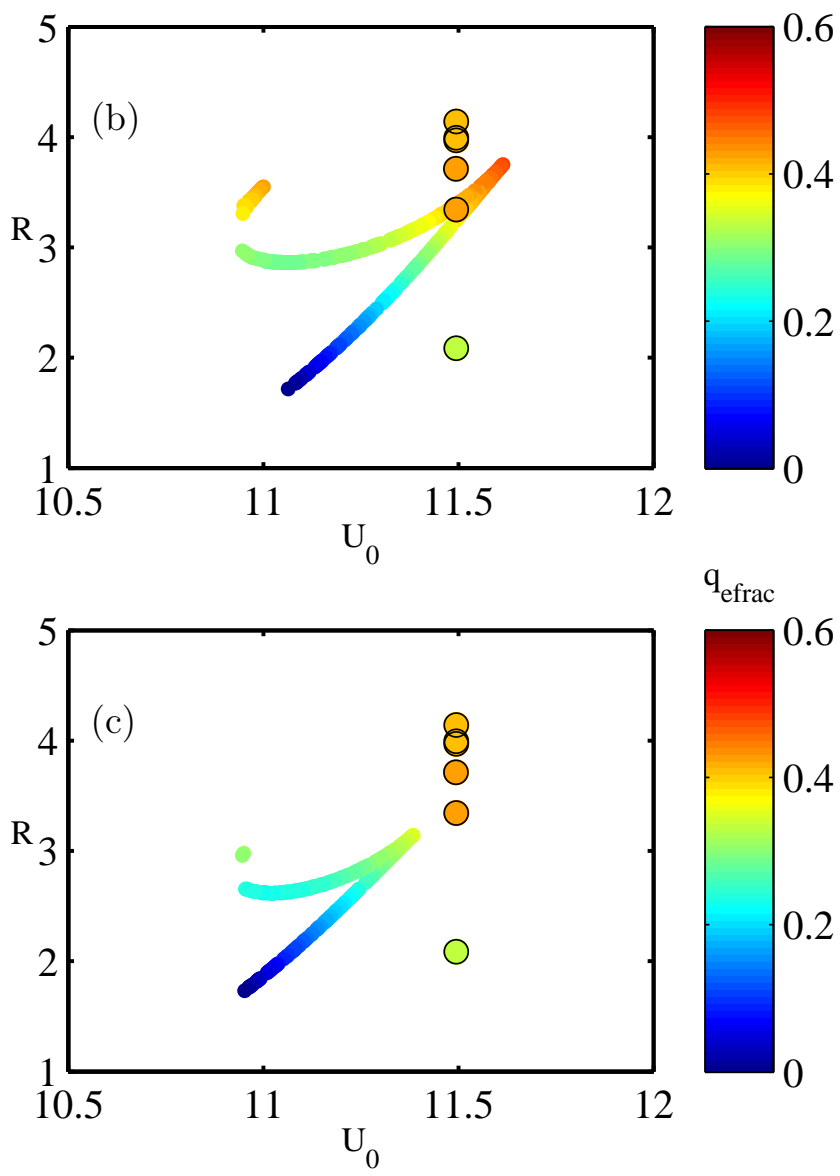

$\mathrm{q}_{\text {efrac }}$

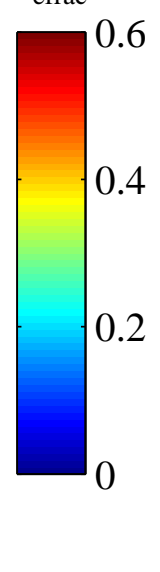

Figure 3-26: Predictions of the modified Wilkinson and Wood (1971) approach. $s=10.8$, $r=0.1$. Topography heights are (a) $h_{b}=2$, (b) $h_{b}=3$, (c) $h_{b}=4$. 


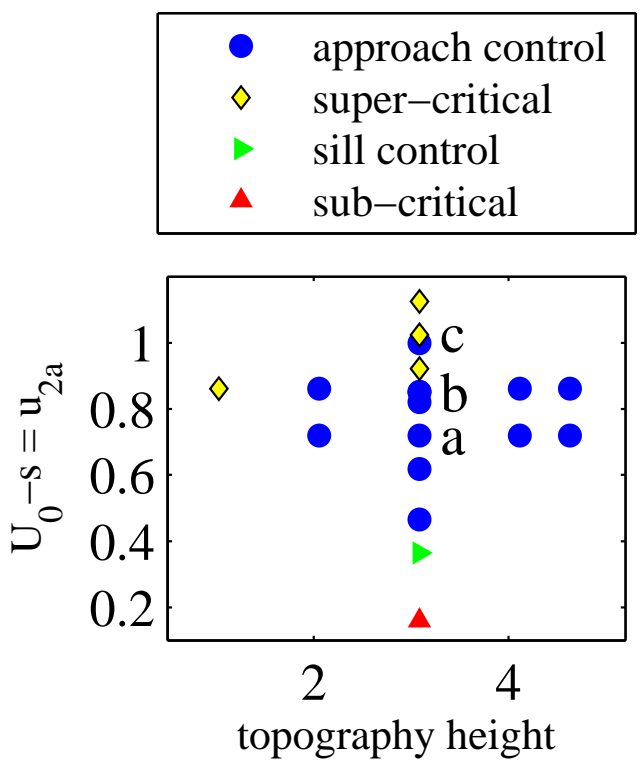

Figure 3-27: Regime diagram of control type. Approach controls are critical overthe topography, upstream of the sill, super-critical flows remain super-critical over the entire domain, sill control flows are critical at the peak of the topography, and sub-critical flows remain sub-critical over the topography. 
(a)
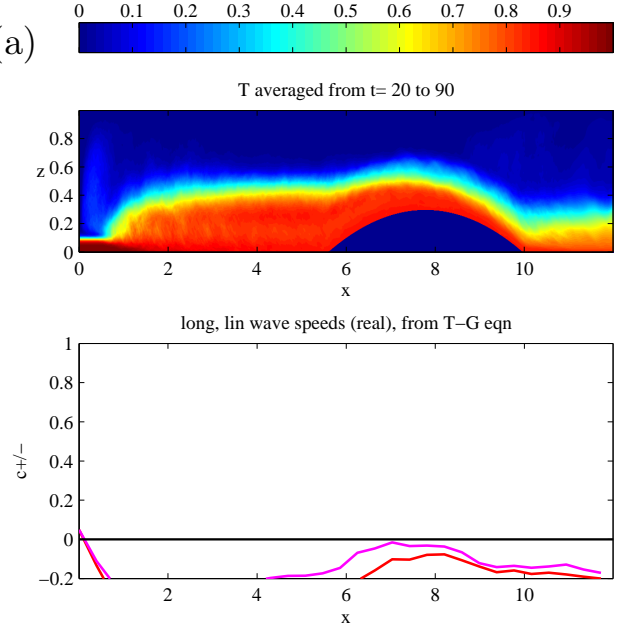

(c)
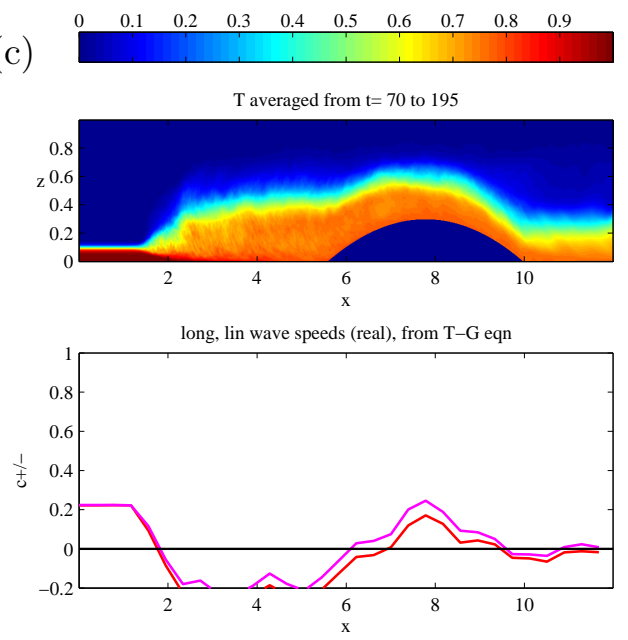

(b)
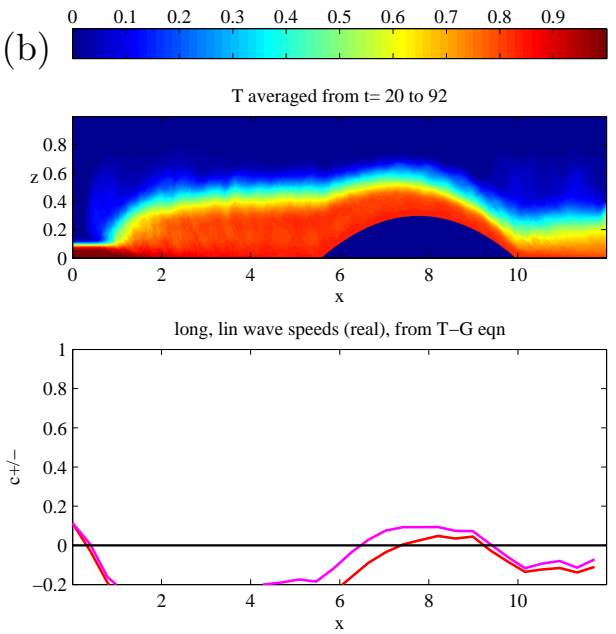

(d)
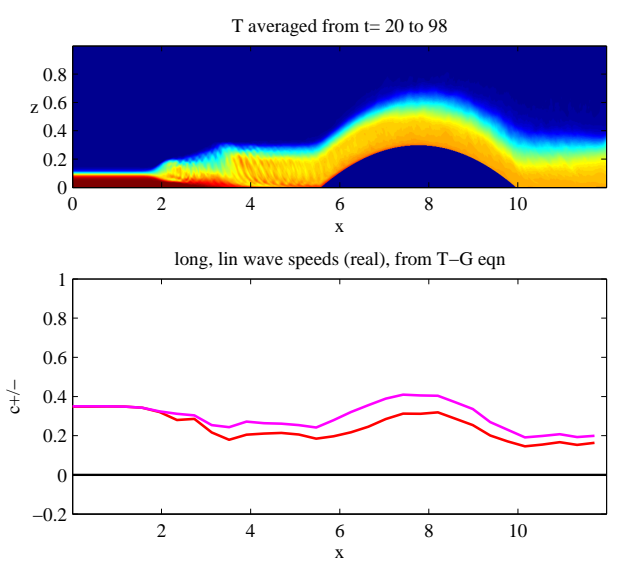

Figure 3-28: Example flows with different control locations. The time averaged scaled density field and $c_{-}$for the first two modes along the domain are shown. Flow is sub-critical when $c_{-}$is negative. $s=8.2, r=0.097$, (a) sub-critical $\left(U_{0}=8.3, R=4.0, q_{\text {efrac }}=0.21\right)$, (b) sill control $\left(U_{0}=8.5, R=4.0, q_{\text {efrac }}=0.23\right)$, (c) approach control $\left(U_{0}=8.9, R=3.7\right.$, $\left.q_{\text {efrac }}=0.37\right)$, (d) super-critical $\left(U_{0}=9.3, R=1.8, q_{\text {efrac }}=0.29\right)$. 


\subsection{Varying Weir Parameters}

The effect of the downstream weir on the flow is considered here. The distance from the inlet to the weir and the height of the weir are varied, and the results are compared to the modified Holland theory (3.2.6, 3.2.7, 3.2.8, 3.2.9, 3.4.9), which does not incorporate these properties into the predictions of jump height and entrainment. The Wilkinson and Wood (1971) theory accounts for the weir height, requiring that the flow be critical at the crest of the weir, as discussed in section 3.4.1. However, these two-layer flows can have flow that is sub-critical over the entire topography, controlled at the crest, controlled upstream of the crest, or super-critical over the topography. Therefore, the precise height of the weir does not determine the jump properties, but instead groups solutions based on whether the flow transitions to subcritical across the jump or remains super-critical over the topography. These solution types are described in $§ 3.4 .1$.

Figure 3-29 shows simulations compared to theory predictions for the three different values of shear of $s=5.2,8.2$, and 10.8, with $r=0.1$. For each shear value, the weir height is varied from 1 to 6 , for a flow with a total depth of 10 . The simulation results gather in two groups: one group of super- to sub-critical transitions for which the flow is controlled $\left(c_{-, 1}=0\right.$, calculated from the continuous profiles using the Taylor-Goldstein equation) at some point over the topography or remains sub-critical over the entire topography, and one group of super- to super-critical transitions for which the flow is not controlled over the downstream topography. The super- to super-critical transitions only occur for the smallest weir height. In this case, the weir is not high enough to force the upstream flow to form a jump to the sub-critical state. The lower layer is instead able to flow over the topography without the flow transitioning to sub-critical. Larger weirs force the upstream flow to transition to sub-critical, although once this adjustment is made, the height of the weir only has a minor influence on the characteristics of the jump.

Figures 3-30 and 3-31a show groups of simulations for which the distance between 


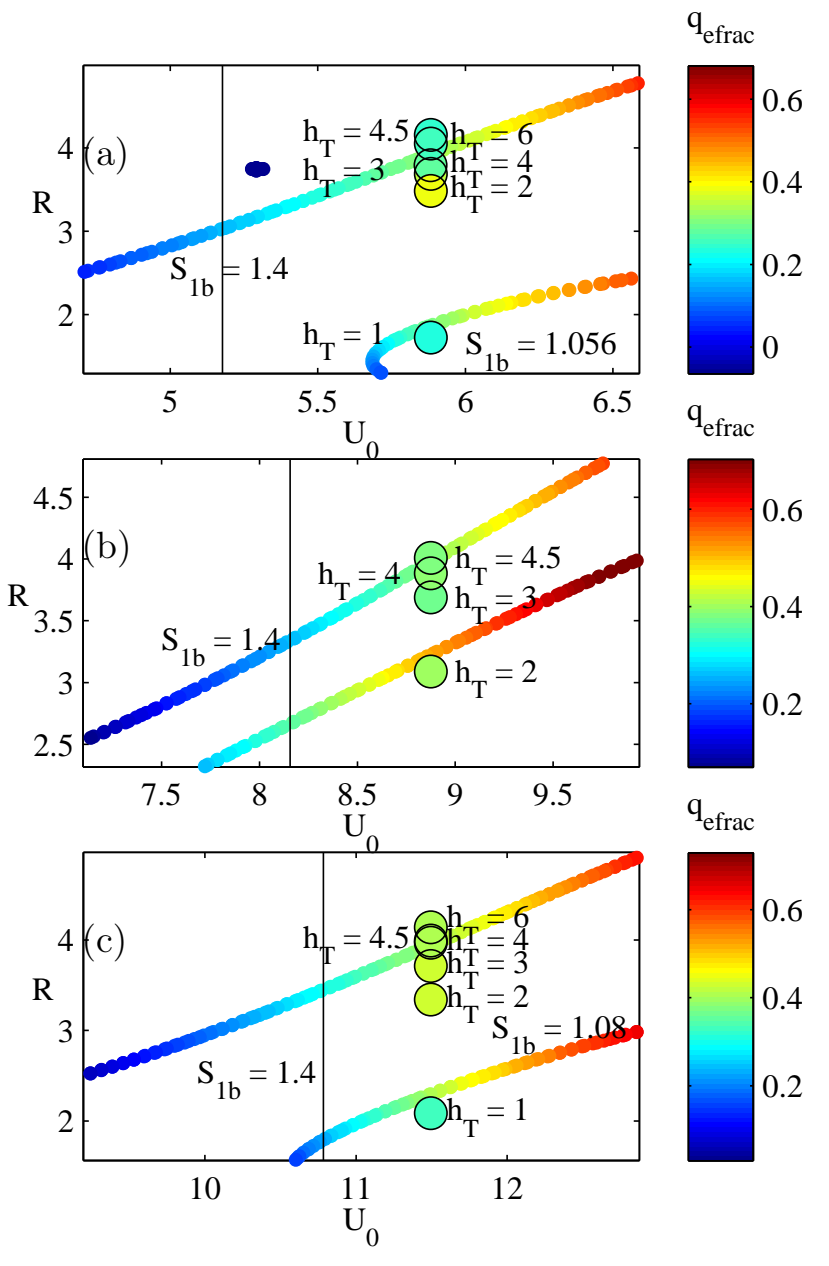

Figure 3-29: Effect of varying weir height from 1 to 6 for (a) $s=5.2$, (b) $s=8.2$, and (c) $s=10.8 . r=0.1$. Circles with borders give numerical simulation results; fill color indicates entrainment calculated from simulation and weir height is labelled for each point. Curve formed by circles without borders gives theoretical predictions; color indicates entrainment predicted by modified Holland approach and $S_{1 b}^{2}$ is labelled for each curve.

the inlet and the weir is varied. For each shear value, super- to sub-critical simulations with distances to the weir of $L=55,110$, and 140 are shown. The results show that the distance to the weir has only a small effect on the jump height and entrainment, with entrainment increasing slightly with distance to the weir. This is consistent with the results of Regev and Hassid (2010), who conducted numerical simulations of a one-and-a-half-layer flow using the commercial CFD package FLUENT, with a k-e turbulence model. Their numerical model simulates an infinitely deep upper layer by using an entraining upper boundary, which allows fluid to exit or enter the numerical domain through the upper boundary. Their simulations indicate that the distance to the weir has a more significant effect on entrainment for smaller distances than those 
considered here because for small distances, the length of the entrainment region can be limited by the distance to the weir. For distances large enough that a roller region develops, entrainment reaches a steady value or decreases slightly as the distance to the weir increases. Their results are shown in figure 3-31b for one-and-a-half layer flows. Their entrainment is given by $\epsilon_{f}=q_{e f r a c}+1$ and the distance between the inlet and the topogarphy, $\frac{L}{h_{1}}$ is equivalent to the non-dimensional $L$ used here. $F r_{1}=U_{0}$, and their upper layer is stagnant for the one-and-a-half-layer flows, giving $s=F r_{1}$. The two-layer simulations shown here are long enough that the entrainment is approximately independent of the distance to the weir, although they see small trends of increasing $q_{\text {efrac }}$ with $L$ for large $U_{0}$ and decreasing $q_{\text {efrac }}$ with increasing $L$ for small $U_{0}$ that are not observed here. The entrainment may be independent of $L$ in two-layer flows because the finite depth limits the vertical extent of the jump, which limits the horizontal extent of the jump if the aspect ratio of the jump is maintained. Also, if the distance to the weir were mush smaller $\left(L=O\left(\frac{1}{r}\right)\right)$, the jump would likely be more significantly affected by the weir.

These results show that, for the parameter ranges considered here, the upstream jump is mostly independent of the downstream weir, except in determining whether the jump is super- to super- or super- to sub-critical. 


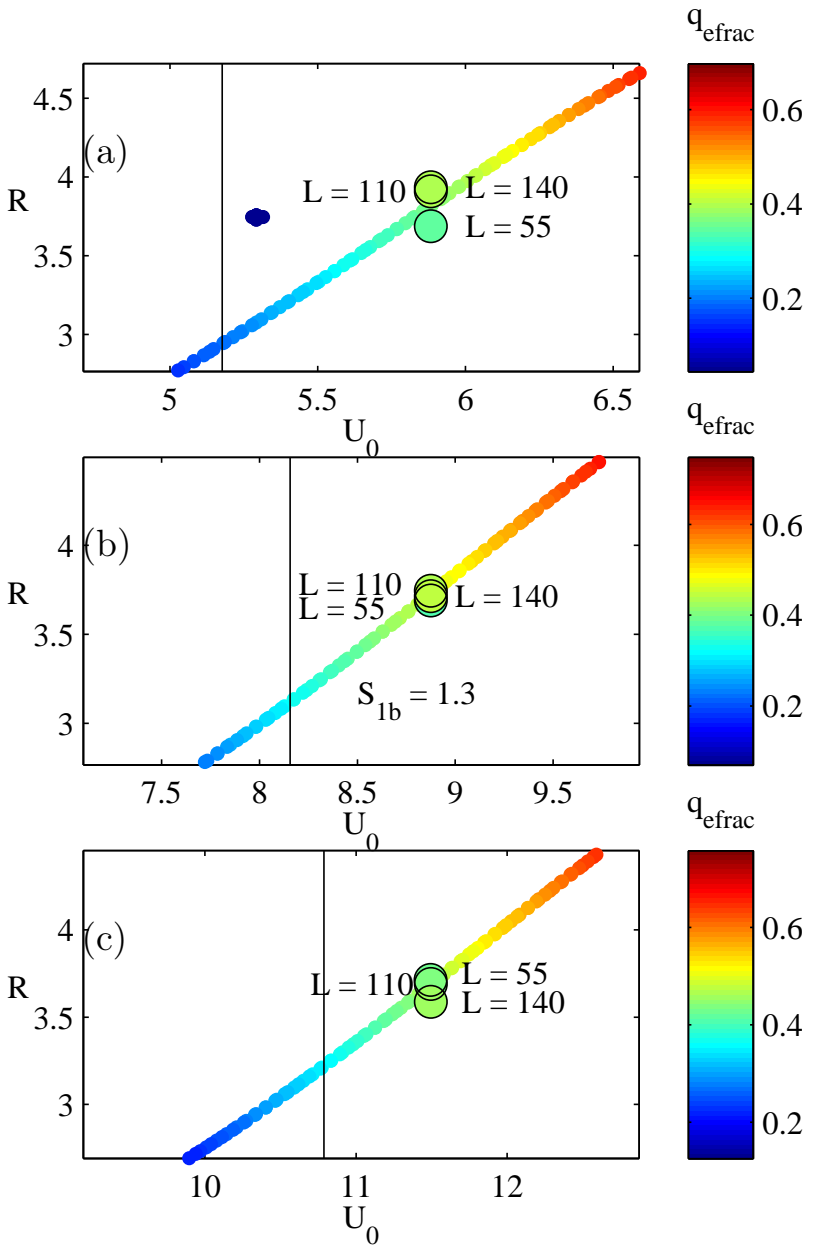

Figure 3-30: Effect of varying distance to weir. Each figure shows three simulation results with distances to the weir of $5.5,11$, and 14. (a) $s=5.2$, (b) $s=8.2$, and (c) $s=10.8$. $r=0.1$.
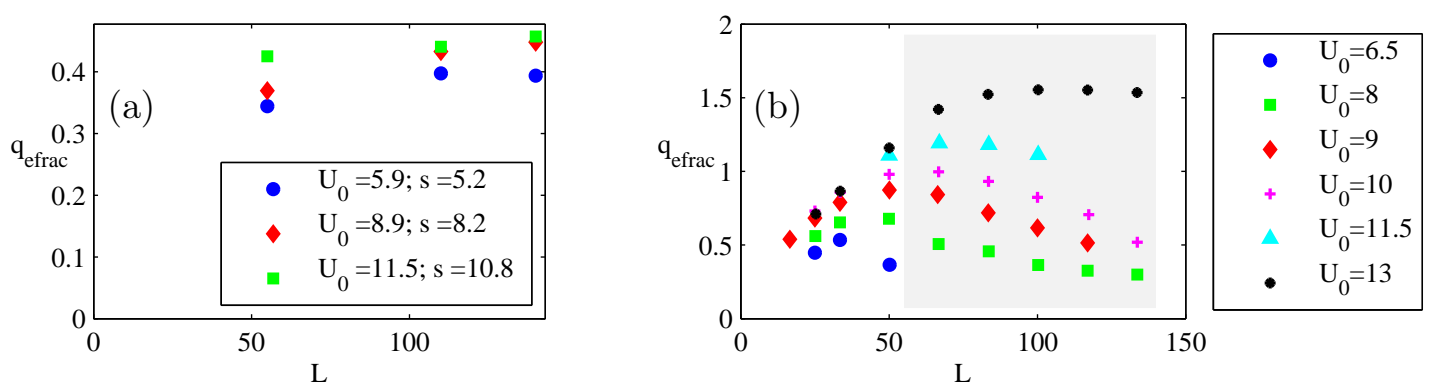

Figure 3-31: Effect on entrainment of varying distance to weir for two-layer flows with $r=0.1$. (b) Shows results from Regev and Hassid (2010) for one-and-a-half layer flow where the upper layer is stagnant so $s=U_{0}$. The shaded region indicates the range of values of $L$ that are considered in this work. 


\subsection{Theory effectiveness}

The two-layer theory with shape parameters $(3.2 .6,3.2 .7,3.2 .8,3.2 .9)$ and the modified Holland et al. (2002) approach for calculating entrainment (3.4.9) can be fit very well with simulations, provided the best shape parameter value is used. Unfortunately, the theory is extremely sensitive to the value of the shape parameter $S_{1 b}^{2}$, particularly for super- to super-critical jumps, and as a result is not useful for predicting jump properties. Although a shape parameter of $S_{1 b}^{2} \approx 1.4^{2}$ is a robust value for super- to sub-critical transitions and $S_{1 b}^{2}=1$ works well for super- to super-critical transitions when the entrainment is specified, the sensitivity to the value of the shape parameter increases significantly when entrainment is calculated as part of the solution.

Although the theory is not very useful for predicting jumps, it nonetheless helps explain the physics of jump. The continuous nature of the velocity profiles is important, as demonstrated by the sensitivity to shape parameters, and entrainment is necessary for high shear jumps. The entrainment also depends on $s^{2}$ because, at the high shear values considered here, the jump begins to behave as an expanding shear layer. However, it can still be analyzed as a hydraulic jump. These results demonstrate the limitations of the two-layer approximation, which fails to predict high shear jumps. The two-layer approximation is only reasonable for low-shear jumps, when the two-layer theory without entrainment or shape parameters (WS, KRS, or VS theories) predicts allowable solutions. When the upstream shear increases beyond this value $(s \approx 3$ for $r=0.1$ ), the flow begins to transition to the high-shear jumps described here, which involve entrainment and have continuous profiles that are not approximately two-layered.

\subsection{Trends in mixing efficiency and entrainment}

The effect of increasing shear on mixing efficiency and entrainment are shown in figure 3-32. As shear increases and entrainment becomes important, mixing efficiency through the jump, defined as the ratio of the cumulative buoyancy flux to the shear 
production,

$$
R i_{f}=\frac{\int_{0}^{x} \int_{0}^{\frac{1}{r}} \overline{w^{\prime} \rho^{\prime}} d z d x}{\int_{0}^{x} \int_{0}^{\frac{1}{r}} \overline{u^{\prime} w^{\prime}} \frac{d U}{d z} d z d x},
$$

decreases. Although the buoyancy flux increases with shear, the shear production increases at a greater rate, resulting in a lower mixing efficiency. The decrease in mixing efficiency occurs through shear values of about $3 \lesssim s \lesssim 8$ for total depth of $D=\frac{1}{r}=10$. These limits approximately correspond to the shear values at which the allowable solution space and then the theoretical solution space are lost. The entrainment increases through these shear values, from $q_{\text {efrac }} \approx 0$ for low shear flows, asymptoting to a value of $q_{\text {efrac }} \approx 0.4$ for larger shears. The super- to sub- critical transitions asymptote to a slightly higher entrainment than the super- to super-critical flows. The transition region for both jump types occurs for $3 \lesssim s \lesssim 8$ and represents a transition from the abrupt, non-entraining $\left(q_{\text {efrac }} \approx 0\right)$ hydraulic jumps described in chapter 2 to a jump that behaves similarly to an expanding shear layer with mixing across the interface and, in these cases, significant entrainment.
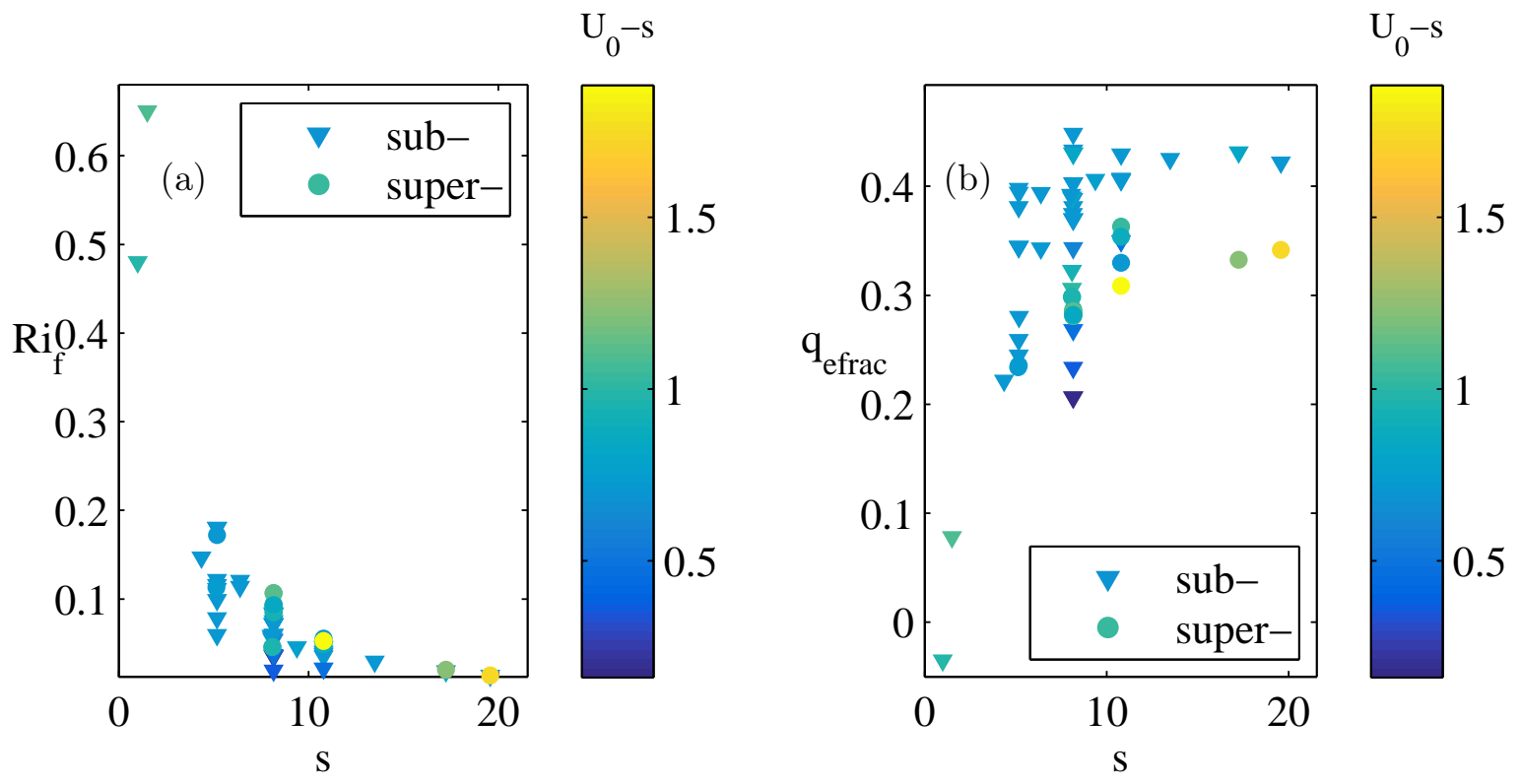

Figure 3-32: (a) Mixing efficiency and (b) entrainment shown for increasing shear. Super- to super-critical and super- to sub-critical jumps are identified by symbol type and $u_{2 a}=U_{0}-s$ is indicated by color. 


\subsection{Comparison with 3D}

The numerical results shown so far in this chapter are all two-dimensional simulations. In chapter $2,3 \mathrm{D}$ simulations were shown to behave similarly to the equivalent $2 \mathrm{D}$ flow, except in the mixing efficiency, which was $R i_{f} \approx 0.55$ for $2 \mathrm{D}$ simulations and $R i_{f} \approx 0.2$ for $3 \mathrm{D}$ results. The jumps were otherwise similar. As the shear increases, however, the 3D simulations diverge from the 2D results, particularly for super- to sub-critical jumps.

In $3 \mathrm{D}$, as the shear increases, the mixed region of the interface extends upward into the fluid until the dense fluid is mixed all the way up to the upper boundary of the flow, and a large recirculating region develops near the upper boundary, just upstream of the sill, as illustrated in figure 3-33. The figure shows super- to sub-critical simulations with $r=0.1$ and $s=5,6.5$, and 8. Figure 3-34 shows velocity and tracer profiles upstream and downstream of the jump for each of the flows shown in figure 333. As the shear increases, the flows transition from having a constant density upper layer, as seen in the low shear jumps (figure 3-34a), to having a continuous, constant stratification throughout the depth (figure 3-34b), and then to having a well-mixed, constant density layer in the interior of the flow (figure 3-34c). These three flow structures are reflected in the downstream density profiles of figures 3-34a through c. The range of shear values through which this occurs corresponds to the range through which the entrainment rapidly increases and the mixing efficiency decreases $(3 \lesssim s \lesssim 8$, figure 3 -32). This is also the range of shear values at which the theoretical solution space is lost for the theories without entrainment or shape parameters.

The difference between the $3 \mathrm{D}$ and $2 \mathrm{D}$ results occurs because the coherent $2 \mathrm{D}$ vortices break down in three dimensions, allowing the fluid to mix more intensely and beyond the extent of the 2D vortices (Peltier and Caulfield, 2003). Peltier and Caulfield (2003) note that 3D mixing begins when 2D vortices reach their maximum amplitude, which, in these flows, appears to be limited by the lower layer depth. When the 3D instability occurs, the fluid is able to mix up farther in the water col- 

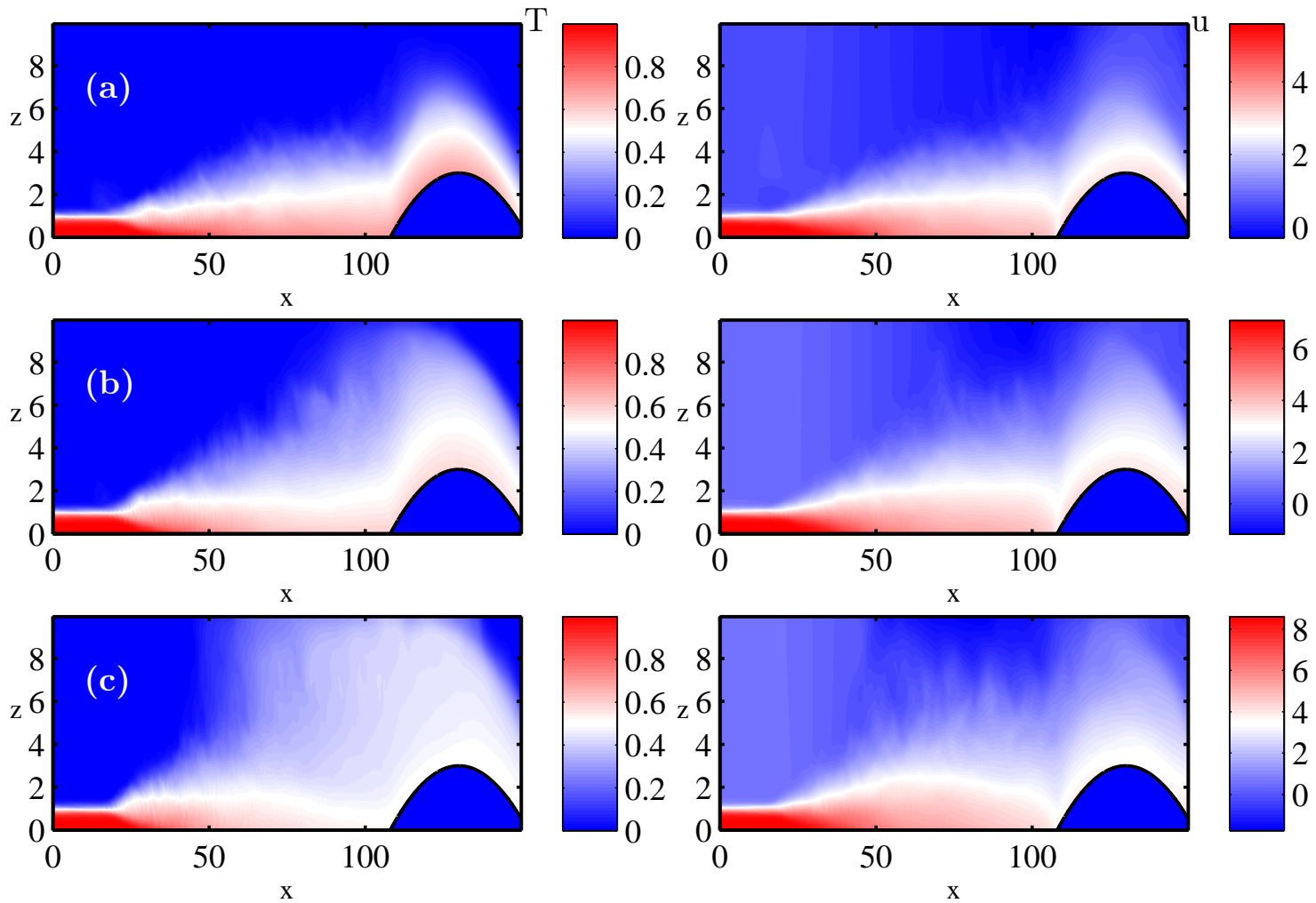

Figure 3-33: Time averaged tracer (left) and velocity (right) fields for 3D super- to subcritical simulation results. (a) $s=5, U_{0}=5.6$, (b) $s=6.5, U_{0}=7.1$, and (c) $s=8, U_{0}=$ 8.6. $r=0.1$.

umn.

For super- to super-critical jumps, a 3D disturbance develops as the inlet velocity in the simulation ramps up, and this disturbance is swept out of the domain as the inlet velocity reaches its steady-state value, leaving only the part of the flow that is similar to the 2D simulations. For super- to sub-critical transitions, however, a region of 3D mixing occurs within the jump and remains ahead of the topography, changing the properties of the jump. The flow develops a strong recirculation region ahead of the jump, with a flow reversal at the top of the domain, as seen in figure 3-34. This provides a strong shear. Conversely, the density mixes throughout the domain, resulting in weak stratification. As a result, the flow does not stabilize, and mixing continues. The gradient Richardson number of the time-averaged flow is shown in figure 3-35, which illustrates that $R i_{g}<\frac{1}{4}$ in the region of the interface for 3D flows. This further demonstrates that, as shear increases, these flows begin to behave more 
as expanding shear layers than hydraulic jumps. It also demonstrates the limitations of the two-layer approximation for high shear flows.

In three dimensions, the density and velocity profiles deviate so significantly from two-layered that approximating them as such is unreasonable. An interface location could still be defined, and entrainment across this interface could be calculated and related to the upstream shear using the modified Holland approach discussed previously. However, the interface location would not have physical significance, and calculating entrainment across this interface would also not be very meaningful. This shows that the two-layered approximation is limited, and cannot reasonably be applied to high shear three-dimensional flows. 

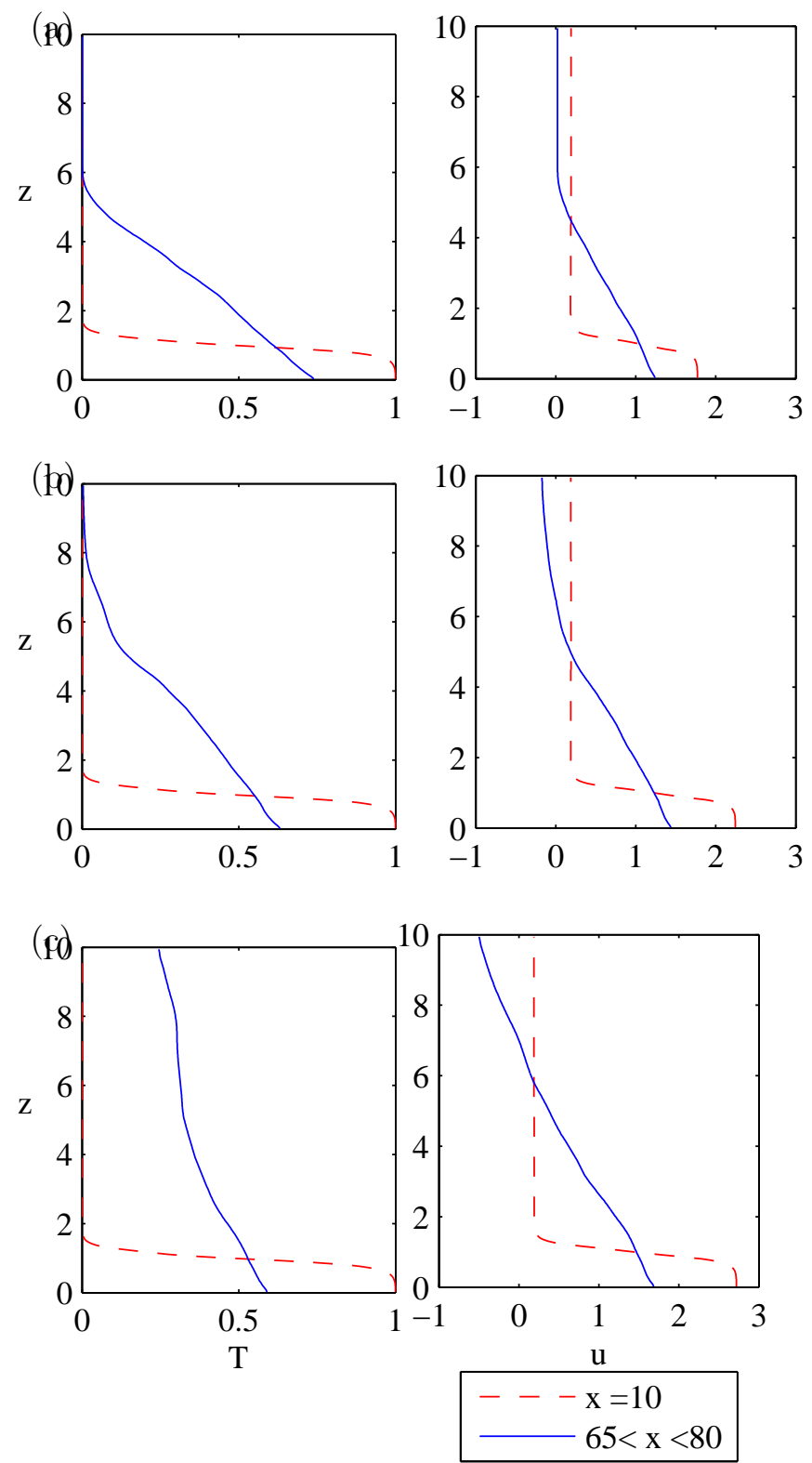

Figure 3-34: Upstream and downstream tracer (left) and velocity (right) profiles from the $3 \mathrm{D}$ simulation results shown in figure 3-33. (a) $s=5, U_{0}=5.6$, (b) $s=6.5, U_{0}=7.1$, and (c) $s=8, U_{0}=8.6 . r=0.1$. 

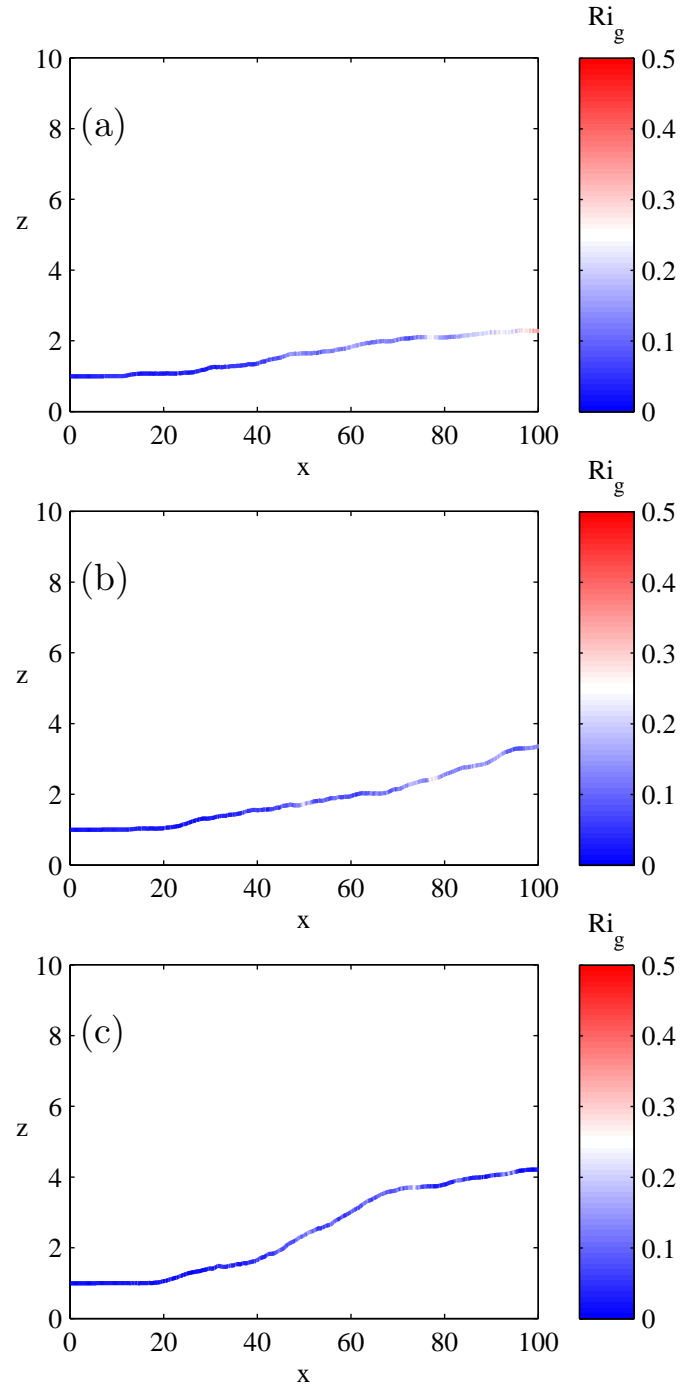

Figure 3-35: $R i_{g}$ and interface location for the 3D simulation results shown in figure 3-33. (a) $s=5, U_{0}=5.6$, (b) $s=6.5, U_{0}=7.1$, and (c) $s=8, U_{0}=8.6 . r=0.1$. 


\subsection{Conclusions}

Basic non-entraining two-layer theories for internal hydraulic jumps fail to provide solutions as the upstream shear increases. First, the allowable solutions, which require that energy be lost across the jump and that the interfacial wave speeds be real, are lost. As shear increases further, even the unphysical mathematical solutions cease to exist. However, previous work by Wilkinson and Wood (1971), Thorpe (2010), and Regev and Hassid (2010) shows that hydraulic jumps in one-and-a-half layer flows with high upstream shear can exist. Numerical simulations also result in jumps in nominally two-layer flows with very high upstream shear. Modifying the two-layer theory to include entrainment and/or shape parameters, which quantify continuous velocity profiles, restores solutions to the theory for these higher shear flows. Both entrainment and shape parameters are required for the theory to be made consistent with the simulations. Shape parameters are particularly important for super- to subcritical solutions with $U_{0}-s=u_{2 a} \lesssim 0.8$, while the values of the shape parameters are all very close to one for super- to super-critical simulations.

Various approaches to predicting the entrainment have been employed for one-anda-half layer flows, such as the energy based approach of Holland et al. (2002) and the flow control approach of Wilkinson and Wood (1971). In this work, it is found that a modification of the Holland et al. (2002) approach is consistent with the numerical simulations. Energy is conserved across the jump, and the energy dissipation in the jump is converted to internal energy to satisfy conservation. The energy dissipation is related to both the turbulent kinetic energy, as proposed by Holland et al. (2002), and, through comparison with calculations, the square of the upstream shear. This indicates that the upstream shear has a controlling influence on the amount of fluid that is entrained into the lower layer. As shear increases, the flow begins to behave similar to an expanding shear layer, although it can still be described as an entraining hydraulic jump.

The modified theory must also include shape parameters for the velocity profile 
because the profiles deviate significantly from a sharp two-layer flow. This is particularly true of the transitions that are super- to super- critical in the two layer limit, but are super- to sub-critical when wave speeds are calculated using the Taylor-Goldstein equation with the continuous velocity and density profiles from the numerical simulations. This inconsistency is related to the velocity minimum that appears near the layer interface in the simulations. A weak velocity minimum can cause a flow that would otherwise be super-critical to become sub-critical. That is, small changes in the shape of the velocity profile can have an important impact on the criticality of flow.

Adding shape parameters, and more importantly entrainment, to the two-layer theory results in jumps predictions at high shears, and these predictions are consistent with the numerical simulations. However, the theory is not very useful for predicting jumps because, when the theory is used to predict both the entrainment and the jump height, it is highly sensitive to the value of the shape parameters, particularly for the super- to super-critical jumps. However, the theory does provide insight into the physics of the jump. The sensitivity to shape parameters indicates that these high shear flows are no longer sufficiently two-layered to apply that approximation. The modified theory also shows that entrainment plays an important role in both super- to super- and super- to sub-critical jumps. Further, the simulations show that the jumps begin to behave like an expanding and entraining shear layer for larger upstream shears. The numerical simulations indicate that this transition occurs in the range of shear values where the basic two-layer theory loses the allowable and then theoretical solutions $(3 \lesssim s \lesssim 8)$.

The numerical simulations show the range of jumps structures that can occur for internal hydraulic jumps with large upstream shear and an active upper layer, which differs from the one-and-a-half layer high shear flows studied previously (Wilkinson and Wood, 1971). These include super-to sub- critical jumps, which have an entrainment and roller region similar to those described by Wilkinson and Wood (1971) and Regev and Hassid (2010), and super- to super-critical jumps, which exhibit an entrainment region but no roller region. These jump structures are very different from 
the jumps found in the low shear flows of chapter 2. Furthermore, the jumps can be controlled at various points over, or not at all by, downstream topography.

The numerical simulations also show how the mixing efficiency is altered by increased upstream shear. There is a transition region between the low and high shear regimes, through which the entrainment increases from zero and the mixing efficiency decreases from about $R i_{f} \approx 0.55$. This transition region also occurs in the range of shear values where the theoretical solutions without entrainment lose the allowable and then theoretical solutions. For larger shear values, the entrainment asymptotes to about $q_{\text {efrac }} \approx 0.4$, with slightly larger values for the super- to sub-critical jumps and slightly smaller values for the super-to super-critical transitions.

Finally, the main results of this section apply to 2D flows. Super- to super-critical transitions in 3D behave similarly. However, super- to sub-critical transitions in $3 \mathrm{D}$ are qualitatively different. As the shear increases, the mixed interface becomes thicker, eventually occupying the entire depth of the fluid. When this occurs, the flow can no longer be reasonable approximated as a two-layer flow, and the theories described in this section are therefore not applicable.

Although most environmental flows in which the lower layer is of comparable depth to the upper layer have lower shear values in the range investigated in chapter 2 , an understanding of how the flows could change if the upstream conditions were modified is important. Furthermore, due to the non-dimensionalization, a flow with two layers that are very close in density effectively provides a high shear flow. This chapter illustrates that as upstream shear increases, entrainment becomes a important physical process and the continuous velocity and density profiles become important, reducing the applicability of the two-layered approximation. 


\section{Chapter 4}

\section{Flow over topography}

\subsection{Introduction}

Hydraulic jumps in the ocean and peripheral channels occur under much more complicated circumstances than those described in chapters 2 and 3. Factors such as topography, stratification, time-dependence, rotation, and three-dimensional effects modify the development, structure, and mixing of these jumps. Table 1.1 summarizes some of the complicating factors of the hydraulic jumps that motivated this work, including friction, rotation, and time-dependence. The purpose of this chapter is to consider more realistic hydraulic jumps with a range of parameters and describe how they compare to the idealized simulations and two-layer theories and how they compare to realistic flows such as Knight Inlet, Hood Canal, and Stellwagen Bank. This analysis can then be used to make predictions about other hydraulic jumps based on a few parameters such as the strength and period of the forcing and the topographic height.

First, the two-layer theory is modified to include a change in topographic height across the jump. This change in depth is assumed to occur instantaneously and at the same location as the jump, as shown in figure 4-1, so that the theory simply connects upstream and downstream states, as in chapters 2 and 3. This modified theory is compared to idealized simulations in which the hydraulic jump occurs over topography. The simulations are otherwise similar to the highly idealized simulations 
of chapters 2 and 3, with an approximately two-layered flow and no time dependence after the jump is fully developed.

More realistic simulations are then conducted to bridge the gap between the highly idealized simulations of chapter 2 and the fairly realistic simulations and observations that have been conducted by others (Farmer and Armi, 1999; Klymak and Gregg, 2004; Cummins, 2000; Lamb, 2004; Afanasyev and Peltier, 2001). More realistic topographies with various heights and downstream slopes are used, and some time-dependence is considered. A more realistic density stratification is applied, and various volume flow rates are simulated. These parameters are varied to cover the range of values found in Knight Inlet, Hood Canal, and Stellwagen Bank, which are the locations that motivated this work. The parameters values for volume flow rate, $q$, topography height, $h_{T}$, tidal perion, $T$, and bottom slope, $S_{l}$, are listed in table 4.1 . While existing work has focused on specific locations, simulating precise details for each locations (Lamb, 2004; Cummins, 2000; Afanasyev and Peltier, 2001), a range of parameters are considered here so that jump behavior in different parameter regimes can be identified. This will allow predictions about the flow in new locations, or how the flow in a given location might change with changing upstream parameters. Some internal hydraulic jump structures that will be identified include the wedge, shear instability versus wave breaking, and transient features versus features of fully developed jumps.

\subsection{Two-layer theory with topography}

The two-layer theory with topography is developed here. The topography is incorporated through an instantaneous change in depth across the jump, depicted in figure 4-1, and hydrostatic pressure against the step is included similar to a form drag. It is very similar to the setup with entrainment shown in figure 3-3, with the addition of a change in depth $(\Delta D)$, giving a new downstream depth of $\frac{1}{r_{b}}$. The theory is first developed using dimensional variables, depicted in figure 2-1. The problem is then 


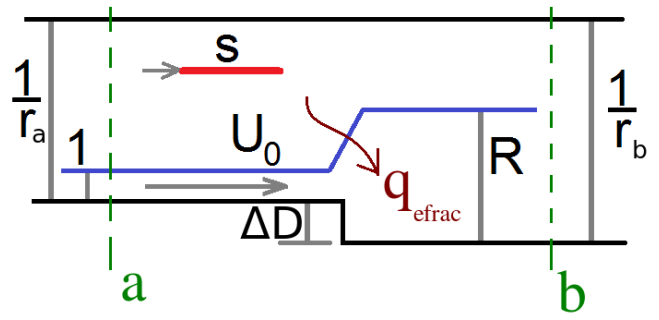

Figure 4-1: Setup of two-layer flow with topography

non-dimensionalized using the same scales as in chapters 2 and 3 (2.2.13). The setup is shown with non-dimensional variables in figure 4-1.

\subsubsection{Theory with instantaneous step}

The momentum flux is conserved as in chapter 2, with the addition of the hydrostatic pressure against the step. Without the hydrostatic pressure against the step, the momentum flux conservation is:

$$
\left[\int_{0}^{D}\left(p+\rho u^{2}\right) d z\right]_{a}^{b}=0 .
$$

In a two-layer approximation, the integral gives:

$$
\begin{aligned}
\int_{0}^{D}\left(p+\rho u^{2}\right) d z= & \int_{0}^{d_{1}}\left(p_{s}+g \rho_{2} d_{2}+g \rho_{1}\left(D-d_{2}\right)-g \rho_{1} z+\rho_{1} u_{1}^{2}\right) d z \\
& +\int_{d_{1}}^{D}\left(p_{s}+g \rho_{2}(D-z)+\rho_{2} u_{2}^{2}\right) d z \\
= & p_{s} D+\left(g \rho_{2} d_{2}+g \rho_{1} d_{1}+\rho_{1} u_{1}^{2}\right) d_{1} \\
& -g \rho_{1} \frac{d_{1}^{2}}{2}+g \rho_{2} d_{2} D+\rho_{2} u_{2}^{2} d_{2}-g \frac{\rho_{2}^{2}}{2}\left(D^{2}-d_{1}^{2}\right) .
\end{aligned}
$$

Note that, for convenience, the coordinate system is redefined so that $z=0$ at the bottom of the domain both upstream and downstream of the jump, and this does not change the integral. The dimensional variables used in (4.2.1) are defined in figure 21, although the total depth changes across the jump $\left(D_{a} \neq D_{b}\right.$, or non-dimensionally, $\left.r_{b} \neq r_{a}\right)$. The momentum flux conservation integral in the two-layer limit is 


$$
\left[p_{s} D+g \rho_{1} \frac{d_{1}^{2}}{2}+g \rho_{2} \frac{d_{2}^{2}}{2}+g \rho_{2} d_{1} d_{2}+\rho_{1} d_{1} u_{1}^{2}+\rho_{2} d_{2} u_{2}^{2}\right]_{a}^{b}=0 .
$$

The hydrostatic pressure drag exerted on the upstream flow by the step is

$$
\int_{-\Delta D}^{0} p_{a} d z
$$

which, in the two-layer approximation gives

$$
\begin{aligned}
\int_{-\Delta D}^{0} p_{a} d z & =\int_{-\Delta D}^{0}\left(p_{s a}+g \rho_{2 a} d_{2 a}+g \rho_{1 a}\left(D_{a}-d_{2 a}-z\right)\right) d z \\
& =\Delta D p_{s a}+g \rho_{2 a} d_{2 a} \Delta D+g \rho_{1 a}\left(D_{a}-d_{2 a}\right) \Delta D+g \frac{\rho_{1 a}}{2} \Delta D^{2}
\end{aligned}
$$

The layer depths in the expression for the hydrostatic pressure against the step could be the upstream depths, the downstream depths, or an average of the two. The upstream depths will be used here because the pressure against the step is added to the upstream side of the momentum flux balance. It will later be shown that this approach produces good quantitative agreement with numerical simulations (figure 43 and 4-3) and very limited use of this theory is made, so other approaches are not considered. The term for the hydrostatic pressure against the step (4.2.3) is added to the upstream side of the momentum conservation equation (4.2.2), giving:

$$
\begin{aligned}
& {\left[p_{s} D+g \rho_{1} \frac{d_{1}^{2}}{2}+g \rho_{2} \frac{d_{2}^{2}}{2}+g \rho_{2} d_{1} d_{2}+\rho_{1} d_{1} u_{1}^{2}+\rho_{2} d_{2} u_{2}^{2}\right]_{a}^{b} } \\
= & \Delta D p_{s a}+g \rho_{2 a} d_{2 a} \Delta D+g \rho_{1 a}\left(D_{a}-d_{2 a}\right) \Delta D+g \frac{\rho_{1 a}}{2} \Delta D^{2} .
\end{aligned}
$$

Entrainment is also included in the theory, and appears in the mass and momentum conservation equations. If entrainment is into the lower layer, the volume conservation equations are

$$
u_{1 a} d_{1 a}\left(1+q_{\text {efrac }}\right)=u_{1 b} d_{1 b}, \quad u_{2 a} d_{2 a}-u_{1 a} d_{1 a}\left(1+q_{e f r a c}\right)=u_{2 b} d_{2 b}
$$

and the mass conservation equation in the lower layer is

$$
u_{1 a} d_{1 a} \rho_{1 a}+u_{1 a} d_{1 a} q_{e f r a c} \rho_{2 a}=u_{1 b} d_{1 b} \rho_{1 b} .
$$


Putting all unknowns in terms of $d_{1 b}$ and $q_{e f r a c}$, which will be specified rather than predicted by the theory, gives:

$$
\begin{gathered}
\rho_{1 b}=\frac{\rho_{1 a}+q_{\text {efrac }} \rho_{2 a}}{1+q_{e f r a c}}, \\
d_{2 b}=D_{b}-d_{1 b}, \\
u_{1 b}=\frac{u_{1 a} d_{1 a}\left(1+q_{e f r a c}\right)}{d_{1 b}}, \\
u_{2 b}=\frac{u_{2 a} d_{2 a}-u_{1 a} d_{1 a} q_{e f r a c}}{D_{b}-d_{1 b}} .
\end{gathered}
$$

Finally, a closure is required to calculate the change in surface pressure across the jump. When entrainment is into the lower layer, the lower layer must lose energy across the jump since this new mass is mixed throughout the lower. This mixing must occur to satisfy the two-layer approximation, which assumes that each layer is homogeneous. Therefore, energy cannot be conserved along the lower streamline, so the KRS theory cannot be applied. Instead, energy is assumed constant along the upper streamline, consistent with the WS theory. Conserving the Bernoulli function along the upper streamline gives

$$
\left[p+\rho g z+\rho \frac{u^{2}}{2}\right]_{a}^{b}=0 .
$$

Since $z$ is constant along the upper streamline,

$$
\left[p+\rho \frac{u^{2}}{2}\right]_{a}^{b}=0
$$

resulting in

$$
p_{s b}-p_{s a}=\frac{\rho_{2}}{2}\left(u_{2 a}^{2}-u_{2 b}^{2}\right)
$$

If entrainment is into the upper layer $\left(q_{\text {efrac }}<0\right)$, the lower layer may still undergo a jump, although the equations change slightly. The momentum flux conservation equation (4.2.4) remains the same. The expressions for volume conservation (4.2.5) also remain the same, while the sign of the entrainment term in them changes. The mass conservation equation changes because the density in the upper layer now 
changes across the jump. Instead of using mass conservation in the lower layer, mass conservation in the upper layer gives a unique equation:

$$
u_{2 a} d_{2 a} \rho_{2 a}-u_{1 a} d_{1 a} q_{e f r a c} \rho_{1 a}=u_{2 b} d_{2 b} \rho_{2 b} .
$$

Putting all unknowns in terms of $d_{1 b}$ and $q_{e f r a c}$ gives:

$$
\begin{gathered}
\rho_{2 b}=\frac{\rho_{2 a} u_{2 a} d_{2 a}-q_{e f r a c} u_{1 a} d_{1 a} \rho_{1 a}}{u_{2 a} d_{2 a}-u_{1 a} d_{1 a} q_{e f r a c}} \\
d_{2 b}=D_{b}-d_{1 b} \\
u_{1 b}=\frac{u_{1 a} d_{1 a}\left(1+q_{e f r a c}\right)}{d_{1 b}} \\
u_{2 b}=\frac{u_{2 a} d_{2 a}-u_{1 a} d_{1 a} q_{e f r a c}}{D_{b}-d_{1 b}}
\end{gathered}
$$

Because fluid mixes into the upper layer, energy can not be conserved along the upper streamline, so energy is instead assumed to be conserved along the lower streamline, giving

$$
p_{s b}-p_{s a}=g \rho_{2 a} d_{2 a}-g \rho_{2 b} d_{2 b}+g \rho_{1}\left(d_{1 a}-d_{1 b}\right)+g \rho_{1} \Delta D+\frac{\rho_{1}}{2}\left(u_{1 a}^{2}-u_{1 b}^{2}\right) .
$$

This model is will be referred to as KRS/WS, and only one of the KRS (energy conserved along lower streamline) or WS (energy conserved along the upper streamline) approaches is appropriate for any given flow. The streamline along which energy is conserved depends on the direction of entrainment.

A change in depth can also be incorporated into the vortex sheet theory. Because the pressure is eliminated from the governing equations by differencing the momentum equations of the two layers, the hydrostatic pressure against the step is not added to the equations. The equations are in flux form, and can therefore be integrated across the jump and the change in depth (Helfrich, 1995). The vortex sheet (VS) theory vorticity equation (2.2.11) is used directly, allowing the total depth $D$ to vary across the jump. Entrainment is also allowed, and the volume conservation equations are modified to account for this. Using (4.2.5) and noting that, because $D=d_{1}+d_{2}$ and 
$q=u_{1} h_{1}+u_{2} h_{2}$

$$
u_{1}=\frac{q+\Delta U\left(D-d_{1}\right)}{D}
$$

where $\Delta U=u_{1}-u_{2}, \Delta U_{b}$ is given by

$$
\Delta U_{b}=\left(d_{1 a} u_{1 a}\left(1+q_{e f r a c}\right) \frac{D_{b}}{d_{1 b}}-q\right) \frac{1}{D_{b}-d_{1 b}}
$$

The downstream density in the entraining layer also changes across the jump according to (4.2.6) if entrainment is into the lower layer or (4.2.7) if entrainment is into the upper layer. This is the Vortex Sheet (VS) theory with entrainment and a change in depth. As with the original VS theory, a closure assumption is not required, except for the assumption that turbulent vorticity terms are negligible. In chapter 3 , the VS theory was not used because the turbulence terms in vorticity balance were significant. It is expected to be applicable here if the shear generated over topography is small, which is found to be true in the numerical simulations described in $\S 4.2 .3$, and if the turbulent vorticity flux term in the vorticity budget is small, which is not necessarily the case (figures 4-3 and 4-4).

\subsubsection{Numerical simulations}

Two-dimensional numerical simulations are conducted using Gerris, described in §3.3. A grid resolution of 256 points in the vertical with isotropic grid cells is used. The simulations are non-dimensionalized in the same was as in chapters 2 and 3 (2.2.13). However, because the jump develops at an a priori unknown location over the to-

pogaphy, the conditions just upstream of the jump $\left(U_{0}, s, r_{a}\right)$ and the characteristic length and velocity scales must be calculated from the simulation results, rather than specified in the setup.

The scaled density field $\left(T=\frac{\rho-\rho_{\min }}{\rho_{\max }-\rho_{\min }}\right)$ at $t=0$, which at $x=0$ is equal to the inlet profile, is shown in figure 4-2. The topography can also be seen in the figure. Topography of height $d_{0}$ is included at the inlet to force upstream shear to develop from a barotropic velocity profile at the inlet. This is similar to jumps in the environment, such as Knight Inlet, where the upstream shear develops due to the 


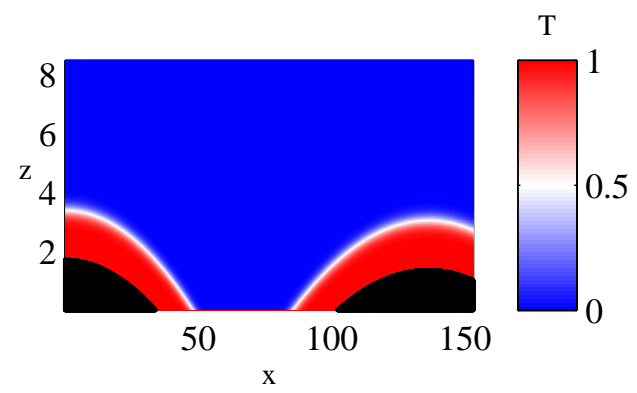

Figure 4-2: Initial scaled density field $T$ for a simulation with a change in topography across the jump. $U_{\text {inlet }}=2.3, d_{0}=1.9, d_{02}=1.5, r_{\text {inlet }}=1.9, c=37, \lambda=3.2$. A jump develops with $s=1.2, r_{a}=0.12, r_{b}=0.11, q_{\text {efrac }}=-0.13$.

stratified flow over topography. Some flows with shear at the inlet were conducted and produced qualitatively similar results to the flows without. The results described here are all barotropic at the inlet and develop upstream shear as the lower layer plunges over the topography. A jump forms over the upstream topography, which results in a change in depth between the upstream and downstream flows, since the jump is not instantaneous (although it is approximated as such in the theory). A downstream topographic feature of height $d_{02}$ helps isolate the jump from the outlet condition. The topography along the entire domain is given by

$$
h_{b}(x)= \begin{cases}d_{0}\left(1-\frac{x^{2}}{c^{2}}\right), & |x|<c \\ d_{02}\left(1-\frac{\left(x-x_{i}\right)^{2}}{c^{2}}\right), & \left|x-x_{i}\right|<c \\ 0, & |x|>c \cup\left|x-x_{i}\right|>c,\end{cases}
$$

where the downstream topography is centered at $x_{i}$ and the half-width of the topographic features is $c$. The topography is depicted in figure 4-2. The initial conditions are $\vec{u}=0$ and

$$
T(x, z, t=0)=\frac{1}{2}\left(1-\tanh \left[\lambda\left(z-\left(r_{\text {inlet }}+f(x)\right)\right)\right]\right),
$$

where

$$
f(x)= \begin{cases}d_{0}\left(1-\frac{x^{2}}{c^{2}}\right), & x \leq \frac{x_{i}}{2} \\ d_{02}\left(1-\frac{\left(x-x_{i}\right)^{2}}{c^{2}}\right), & x>\frac{x_{i}}{2} .\end{cases}
$$

The inlet conditions for $T$, the scaled density field, and $u$, the horizontal velocity, 
are hyperbolic tangent profiles very similar to those defined in $\S 3.3$, except that the profile is defined in terms of $z-d_{0}$ instead of $z$, where $d_{0}$ is the height of the topography at the inlet. The inlet velocity profile is given by

$$
u(x=0)= \begin{cases}U_{\text {inlet } \frac{1}{2}\left(1+\cos \left(\frac{t}{4}+\pi\right)\right),} & t \leq 4 \pi \\ U_{\text {inlet }}, & t>4 \pi\end{cases}
$$

and the inlet scaled density profile is

$$
T(x=0)=\frac{1}{2}\left(1-\tanh \left[\lambda\left(z-d_{0}-r_{\text {inlet }}\right)\right]\right)
$$

where $r_{\text {inlet }}$ is the non-dimensional lower layer thickness at the inlet.

\subsubsection{Theory compared to simulations}

Two-layered theoretical solution curves are shown in the top left panel of figures 4-3 $\left(s=1.2, r_{a}=0.12, r_{b}=0.11, q_{\text {efrac }}=-0.13\right)$ and $4-4\left(s=1.0, r_{a}=0.38, r_{b}=\right.$ $\left.0.31, q_{\text {efrac }}=-0.04\right)$. Solutions to both the KRS/WS and VS theories are plotted. Solutions with entrainment and a change in depth are compared to solutions with entrainment but no change in depth. The change in depth shifts the solution curves up, resulting in larger jumps. This occurs because the downstream depth is larger, so the jump can also be larger. The curves in figure 4-4 are plotted for a larger change in depth, and show that the change also shifts jump solutions to smaller values of $U_{0}$.

The time-averaged scaled density fields of two numerical simulations are depicted in the top right panel of figures $4-3\left(U_{\text {inlet }}=2.3, d_{0}=1.9, d_{02}=1.5, \quad r_{\text {inlet }}=\right.$ $1.9, c=37, \lambda=3.2)$ and $4-4\left(U_{\text {inlet }}=0.81, d_{0}=0.65, d_{02}=0.52, r_{\text {inlet }}=1.9, c=\right.$

$9.2, \lambda=11)$. The parameters of the setup are all very different between the two simulations because they are non-dimensionalized based on the lower layer thickness just upstream of the jump, which cannot be directly controlled. The distance between the topographic features was also intentionally varied between the simulations to test the theory under different conditions. The simulations produce jumps with 
upstream conditions that match the theoretical curves that are also shown in figures 4-3 and 4-4. The lower left panels of figures 4-3 and 4-4 show the upstream and downstream-averaged velocity and scaled density profiles. In both cases, the flow over the initial topographic feature develops upstream shear, and a jump forms over the topography. Because the upstream flow develops over the topography, the upstream profiles are not as close to two-layered as those in chapters 2 and 3, although they are reasonably close. Downstream, the profiles deviate more significantly from two-layered, particularly the velocity profiles, which both develop minima near the density interface. The downstream topography forces the flow to return to a supercritical state, as shown by the wavespeeds in the middle right panel of the figure, which isolates the jump from the (imperfect) outlet.

The numerical results are compared to the theory in the top left panels of figures 4-3 and 4-4. Both the KRS/WS and VS theories predict the correct trend; that is, the theory with a change in depth and the simulations with topography both move away from the original theory curves in the same direction. However, the theory with a change in depth is not accurate enough to make predictions about a jump, particulaly since the location of the jump would typically be unknown beforehand, so the change in depth across the jump and the upstream conditions would also be unknown. The theory does indicate how that change in depth will affect the jump, which is to increase the jump height compared to a flow without an increase in depth.

Entrainment is $q_{\text {efrac }}=-0.13$ for the jump shown in figure 4-3 and $q_{\text {efrac }}=-0.04$ for the jump in figure 4-4. The magnitude of the entrainment is smaller than in the high shear flows of chapter 3 , and the entrainment is typically negative (into the upper, inactive layer), unlike the high shear flows. However, the entrainment is small, and the shear values are consistent with the low shear simulations described in chapter 2 .

The vorticity budgets for the two simulations are shown in figure 4-3 and figure 4-4 in the lower right panel. In figure 4-3, the vorticity budget shows that the turbulent 

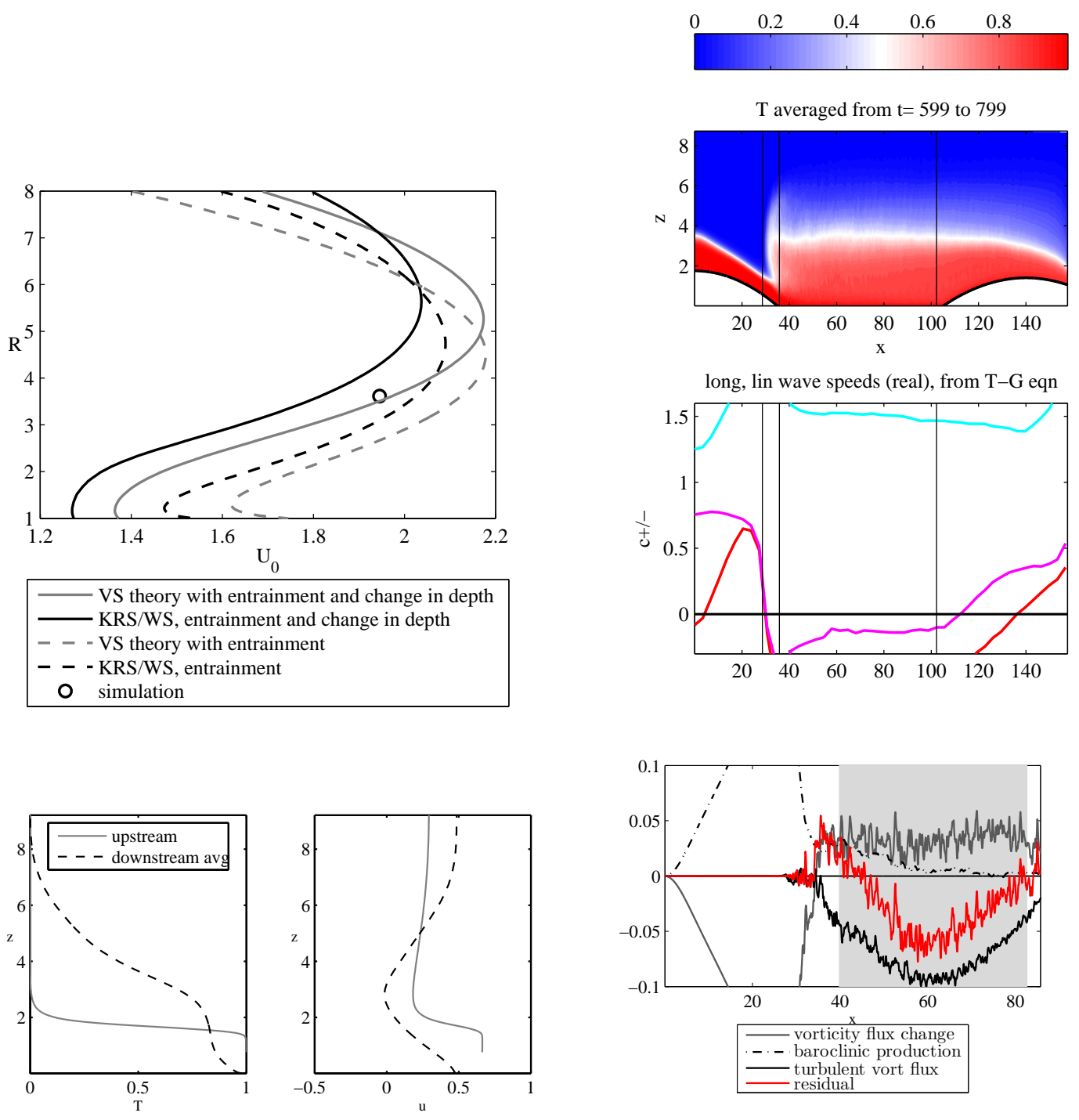

Figure 4-3: Theory with change in depth compared to numerical simulations. The simulation setup parameters are $U_{\text {inlet }}=2.3, d_{0}=1.9, d_{02}=1.5, r_{\text {inlet }}=1.9, c=37, \lambda=3.2$. A jump develops with $s=1.2, r_{a}=0.12, r_{b}=0.11, q_{\text {efrac }}=-0.13$. Upper left panel shows KRS/WS and VS theories with entrainment and change in depth and curves with entrainment and no change in depth. The simulated jump is also indicated. The upper right panel shows the time-averaged tracer field for a fully developed jump over topography. Below that, $c_{ \pm}$for the first two baroclinic modes (calculated from the continuous density and velocity profiles using the Taylor-Goldstein equation in the long wave limit) are shown along the domain. Three vertical lines indicate the upstream location and edges of the downstream averaged location. The lower left panels show upstream and downstream-averaged velocity and tracer profiles. The lower right panel shows the cumulative integral of the terms in the vorticity budget. 

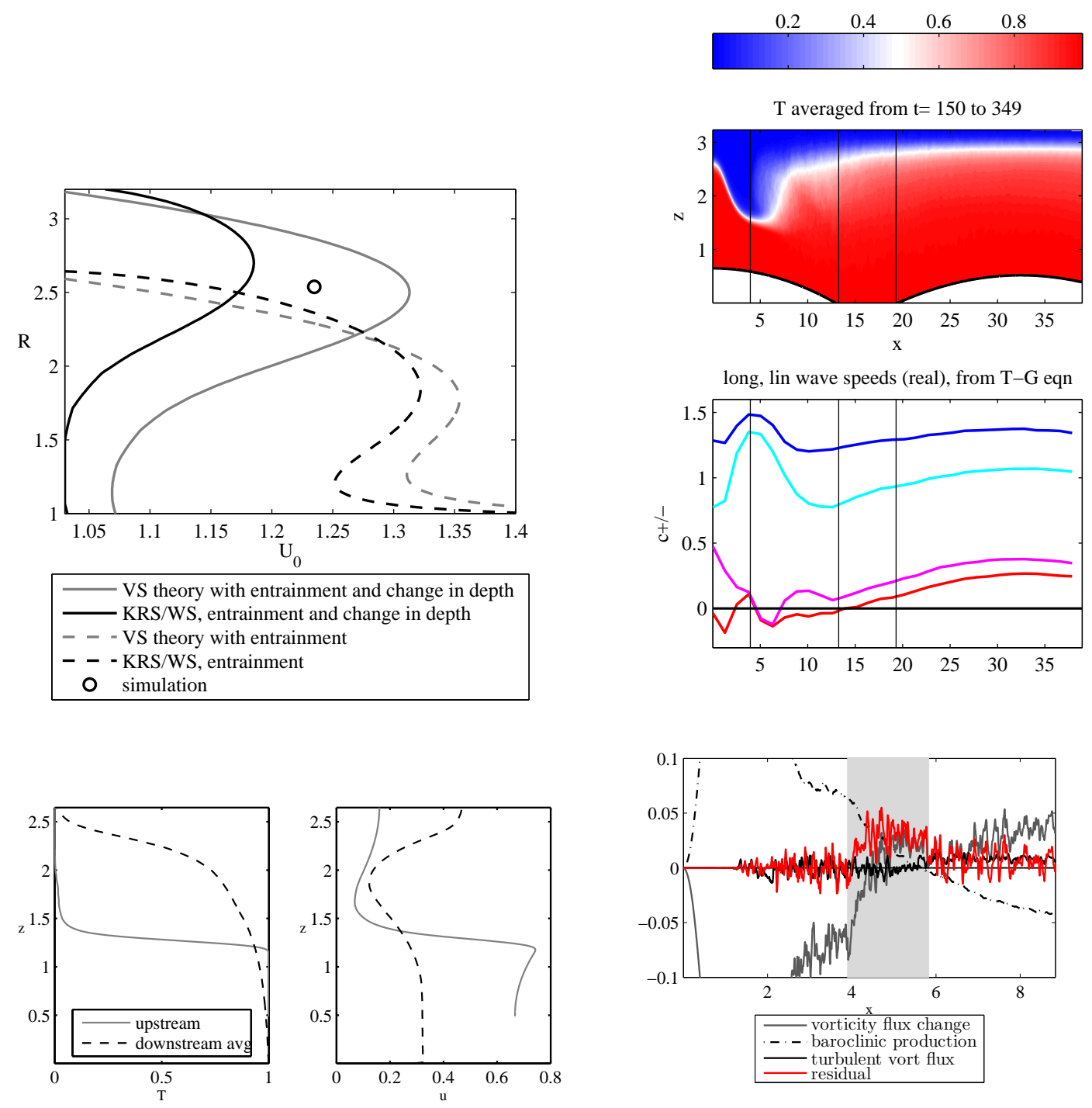

Figure 4-4: Theory with change in depth compared to numerical simulations. Simulation setup parameters are $U_{\text {inlet }}=0.81, d_{0}=0.65, d_{02}=0.52, r_{\text {inlet }}=1.9, c=9.2, \lambda=11$. A jump with $s=1.0, r_{a}=0.38, r_{b}=0.31, q_{\text {efrac }}=-0.04$ develops. Upper left panel shows KRS/WS and VS theories with entrainment and change in depth and curves with entrainment and no change in depth. The simulated jump is also indicated. The upper right panel shows the time-averaged tracer field for a fully developed jump over topography. Below that, $c_{ \pm}$for the first two baroclinic modes (calculated from the continuous density and velocity profiles using the Taylor-Goldstein equation in the long wave limit) are shown along the domain. Three vertical lines indicate the upstream location and edges of the downstream averaged location. The lower left panels show upstream and downstream-averaged velocity and tracer profiles. The lower right panel shows the cumulative integral of the terms in the vorticity budget. 
vorticity flux is large, as is the residual. The large turbulent term is consistent with the moderate magnitude of the entrainment, and suggests that the VS theory may be less applicable. However, the comparison of the simulation to the theories indicates that both theories, and in particular the VS theory, actually agree fairly well with the simulation results. The flow shown in figure 4-4 has a smaller turbulent vorticity flux term, which is consistent with the low value of entrainment. The simulation falls between the two theory curves, agreeing reasonably well with the VS theory. The residual is also large in the region downstream of the jump, between the two topographic features.

The two-layer theories are less useful when significant topography is included because the location of the jump, and therefore the upstream parameters, are not clear given the conditions upstream of or at the sill. While the theories compare reasonably well when the upstream parameters are calculated from the simulations, applying the theories to jumps in the ocean would be difficult. Furthermore, the two-layer theories do not provide any information about the qualitative jump types or mixing. For these reasons, the two-layer theories are abandoned here, and numerical simulations alone are considered throughout the remainder of this chapter.

\subsection{Simulation of more realistic flows}

The two-layer approximation fails in more complicated flows, such as those with large upstream shear or topography. Hydraulic jumps in the environment include topography, as well as other complicating factors such as time-dependent forcing, continuous density stratification, and bottom friction. Furthermore, additional jump structures such as a wedge, overturning wave, and upstream shear instability have been discussed in the context of Knight Inlet (Armi and Farmer, 2002; Lamb, 2004), and these jump structures, particularly the wedge, have not been identified in the very idealized sim-

ulations from chapters 2 and 3 and $\S 4.2$. To help understand these flows without the use of two-layer theories, this section investigates the qualitative types of flows that 
may arise when these parameters are varied, and how mixing is affected. The results are considered in the context of the existing flows in Knight Inlet, Stellwagen Bank, and Hood Canal, and they are analyzed in light of the results from chapters 2 and 3.

\subsubsection{Simulation setup}

More realistic 2D flows are simulated using Gerris (§3.3), and 3D results are later considered in $\S 4.3 .7$. These simulations are non-dimensionalized using the maximum total depth (away from the topography) of the flow as the lengthscale, rather than the upstream lower layer depth, as was used in chapters 2 and 3, because the depth of the lower layer changes between the inlet and the jump, and is unknown before the jump develops. The grid resolution is 128 points in the vertical, which is coarser than the previous simulations. However, the resolution is similar to that of previous work (Lamb, 2004), and some simulations with a resolution of 256 points in the vertical show consistent results.

A barotropic flow is forced over topography to simulate the tidal forcing of flow over a sill as occurs in Knight Inlet, Hood Canal, and Stellwagen Bank. The shape, shown in figure 4-5, is simplified but based on the topography of the sill in Hood Canal (figure 1-3):

$$
h_{b}(x)= \begin{cases}h_{T} e^{\left(\frac{\left(x-x_{0}\right)}{\mu}\right)^{2}} & x \leq x_{0} \\ h_{T} e^{\left(\frac{\left(x-x_{0}\right)}{\nu}\right)^{2}} & x>x_{0} .\end{cases}
$$

A downstream sill is not included because the correct shape is unknown, and the background density stratification is used to provide a downstream reservoir condition to control where the jump occurs on the topography. This approach allows disturbances to propagate from the outlet to the jump, so the simulation results are only valid before these disturbances reach the region of the jump, and this is considered when analyzing the simulation results. The upstream stratified flow specified at the inlet and at the initial time (figure 4-5) has a hyperbolic tangent density interface 
given by

$$
T(z)=\frac{1}{2}\left(1-\tanh \left(\lambda\left(z-z_{0}\right)\right)\right)
$$

The barotropic upstream and initial velocity is

$$
u(z, t)= \begin{cases}U_{\text {inlet }} \sin \left(\frac{2 \pi t}{T_{T}}\right), & t \leq \frac{T_{T}}{4} \\ U_{\text {inlet }}, & t>\frac{T_{T}}{4}\end{cases}
$$

The flow over topography causes a shear to develop, and as the flow spills over the sill, a hydraulic jump forms downstream of the sill. The magnitude of the barotropic flow increases with time on a timescale similar to the M2 tidal period, which is the dominant tidal constituent in Knight Inlet, Hood Canal, and Stellwagen Bank. The first quarter of the tidal cycle is simulated, and the velocity is held at the maximum value for $t>\frac{T_{T}}{4}$. This allows some transient effects to be observed. Once the maximum velocity is reached, it is maintained to allow a fully developed jump to form. Although the tide fluctuates continuously, a steady jump is required to analyze turbulent statistics and averaged fields, which is why the flow is forced with the maximum velocity for $t>\frac{T_{T}}{4}$.

Downstream, the flow exits through a open boundary condition of $p=p_{\text {hydros }}$, as used in chapter $2(\S 2.3)$. Due to the deeper lower layer of dense fluid, a boundary condition of $p=0$ cannot be used because the denser fluid would spill out too quickly, causing a bore to propagate into the domain along the upper boundary. The bottom boundary condition is typically free-slip over the topography, although a no-slip condition with viscosity near the bottom of the domain is considered and discussed further in $§ 4.3 .6$. The upper boundary is a free-slip rigid lid.

\subsubsection{Parameter space of realistic flows}

The simulations are guided by the characteristics of hydraulic jumps in the environment (described in $\S 1.1$ ), and the parameters that are varied include the volume flow rate $q=U_{\text {inlet }}\left(1-h_{b, \text { inlet }}\right)$, the maximum topographic height $h_{T}$, the length of the 

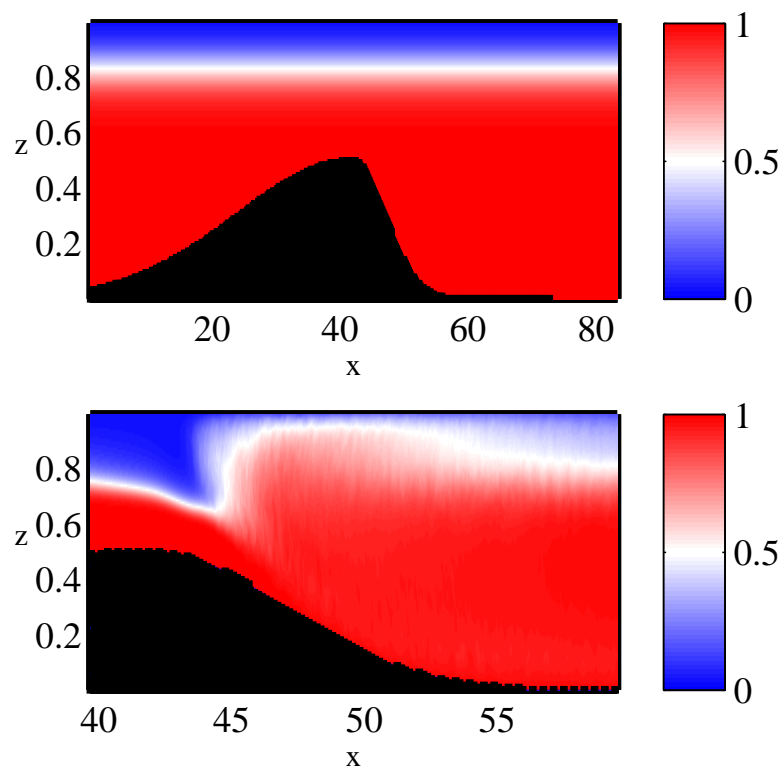

Figure 4-5: Initial conditions and topography. $T=\frac{\rho-\rho_{\min }}{\rho_{\max }-\rho_{\min }}$ at $t=0$, showing full domain and topography (top), and time averaged (from $t=160$ to $t=320$ with data saved at intervals of $\Delta t=2) T$ field zoomed in on the jump. Intermediate instantaneous $T$ fields showin the jump development can be seen in figure 4-7.

non-dimensional tidal period, $T_{T}$, and the slope of the downstream topography, $S_{l}$. The characteristic length scale used to non-dimensionalize the problem is the total depth of the domain, away from the topography, and the characteristic velocity scale incorporates the reduced gravity $\left(\hat{U}=\sqrt{g^{\prime} H}\right)$. The resulting characteristic time scale is $\hat{T}=\sqrt{\frac{H}{g^{\prime}}}$. Varying the non-dimensional tidal period is therefore equivalent to varying the total depth of the flow or the stratification, since all of the motivating environmental jumps are forced at the M2 tidal period of $12.42 \mathrm{hr}$. The volume flow rate is noted instead of the barotropic velocity because the topographic height at the inlet $\left(h_{b, \text { inlet }}\right)$ depends on $h_{T}$, and both are varied. Non-dimensional values of each of these parameters are given in table 4.1 for Knight Inlet, Stellwagen Bank, and Hood Canal. Typical values used in the simulations are $0.03 \lesssim q \lesssim 0.25,0.5 \lesssim h_{T} \lesssim 0.7$, $380 \leq T_{T} \leq 1200$, and $0.02 \lesssim S_{l} \lesssim 0.09$, which are in the range of slope values found in Knight Inlet, Stellwagen Bank, and Hood Canal (table 4.1).

Other simulation parameters, such as the density stratification and bottom friction are also considered. The density stratification in the simulations is typically 


\begin{tabular}{llll}
\hline & Knight Inlet & Stellwagen Bank & Hood Canal \\
\hline$q$ & 0.033 & 0.087 & 0.18 \\
$h_{T}$ & 0.7 & 0.65 & 0.5 \\
$T$ & 1203 & 819 & 379 \\
slope $S_{l}$ & $0.05-0.2$ & $0.003-0.04$ & 0.03 \\
\hline
\end{tabular}

Table 4.1: Non-dimensional parameters of observed hydraulic jumps, calculated from data in Klymak and Gregg (2003); Lamb (2004); Gregg and Pratt (2010); Scotti et al. (2007).

approximately two-layered, with a hyperbolic tangent interface. The interface thickness is varied. This is a simplified stratification compared to the profiles seen in the environment.

\subsubsection{Flow structure}

The resulting jumps can be categorized into a few qualitative types. These types include a higher-mode jump that develops a wedge region, a first-mode jump with wave overturning, and jumps with upstream shear instabilities. Jumps with upstream shear instabilities can be higher mode jumps or first-mode jumps with shear instabilities along the interface upstream of the jump. First-mode jumps with shear instabilities can develop an undular bore that propagates to the front of the jump and then deteriorates into downstream shear instabilities. The mode is determined by the internal long wave speeds calculated from the Taylor-Goldstein equation (Appendix B): firstmode jumps are super-critical to all modes upstream of the jump, whereas $n$ th-mode jumps are sub-critical to $n$-1-mode waves and super-critical to $n$ th-mode and higher mode waves upstream of the jump. Examples of these jumps types are shown in figures 4-6, 4-7, 4-8 and 4-9.

Higher mode jumps with a wedge of stagnant, homogeneous, intermediate density fluid (figure 4-6) represent a new jump structure that can be analyzed due to the topography. Without topography, a higher-mode jump would propagate away or deteriorate into a rarefaction wave. While the jump is only quasi-steady since mode-one disturbances can propagate upstream, the topography provides a reasonable frame of 
reference from which to analyze it, which is why it did not appear in chapters 2 or 3. An distinctive characteristic of the higher-mode jump is the upward deflection of isopycnals near the surface, just ahead of the jump. Isopycnals deeper in the water plunge over the topography, and a region of stagnant, homogeneous fluid is enclosed by these diverging isopycnals. This is the wedge region, which occurs just ahead of the jump itself. Farmer and Armi (1999) suggest that the wedge acts as a passive upper layer, allowing the lower layer to be analyzed using single layer hydraulics with reduced gravity. In this analysis, the layer approximation may indicate a super-critical flow, while wave speeds calculated from the continuous density and velocity profiles through the entire depth of the flow indicate the jump is of a higher-mode.

Mode-one jumps with wave overturning (figure 4-7) occur at higher volume flow rates. In these jumps, all isopycnals plunge downward over the topography and deflect up in a wave that subsequently overturns. As the wave overturns, shear instabilities on the wave develop. The jumps are qualitatively similar to fully turbulent jumps with overturning at the leading edge, described in chapter 2. Jumps with upstream shear instability (figure 4-8 and 4-9) look more like the high shear jumps from chapter 3 because they both have unstable upstream shear layers. However, entrainment is smaller in these lower shear jumps $\left(q_{\text {efrac }}=-0.034\right.$ for figure $4-8$ and $q_{\text {efrac }}=0.037$ for figure 4-9). Furthermore, while shear instabilities form upstream of the jump due to the thin interface, the gradient Richardson number increases above $\frac{1}{4}$ near the interface downstream of the jump.

Which jump type occurs depends on the paramters of the flow, such as the volume flow rate, stratification, and topography. The regime diagrams in figures 4-10, 4-12, and 4-11 show for what parameter ranges each jump type occurs. The mode of the jump is determined after a fully developed jump is formed, since all flows are sub-critical initially and when the forcing is small. However, the time evolution of the jump shows whether wave overturning (identified by streamlines tilting to vertical) or upstream shear instabilities occur first in mode-one jumps, and whether a wedge (identified by streamlines deflecting upward without mixing) or upstream shear in- 

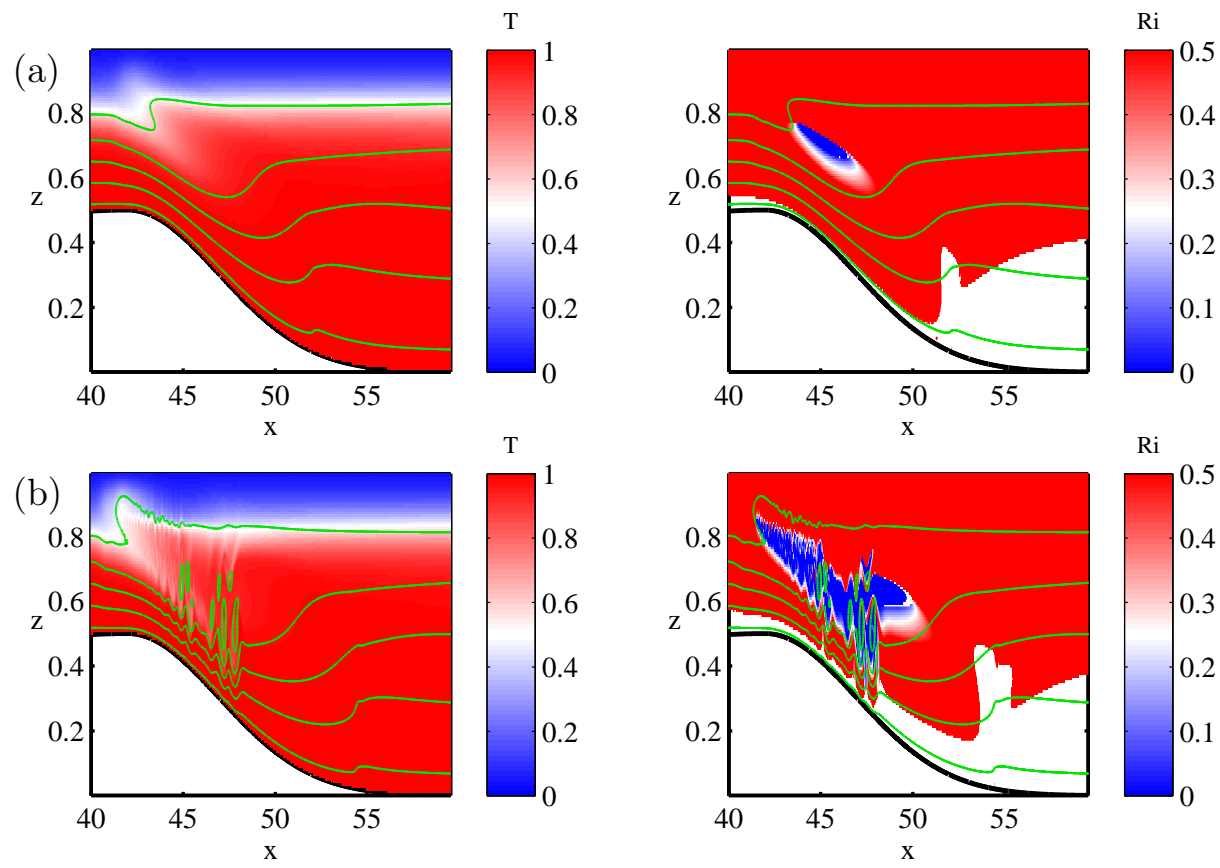

Figure 4-6: Higher-mode jump with wedge. $q=0.05, h_{T}=0.5, T_{T}=380$, two-layer stratification, and $\nu=7$ (defined in (4.3.1)). (a) $t=138$, (b) $t=178$. Green lines in plots of $R i$ are streamlines.
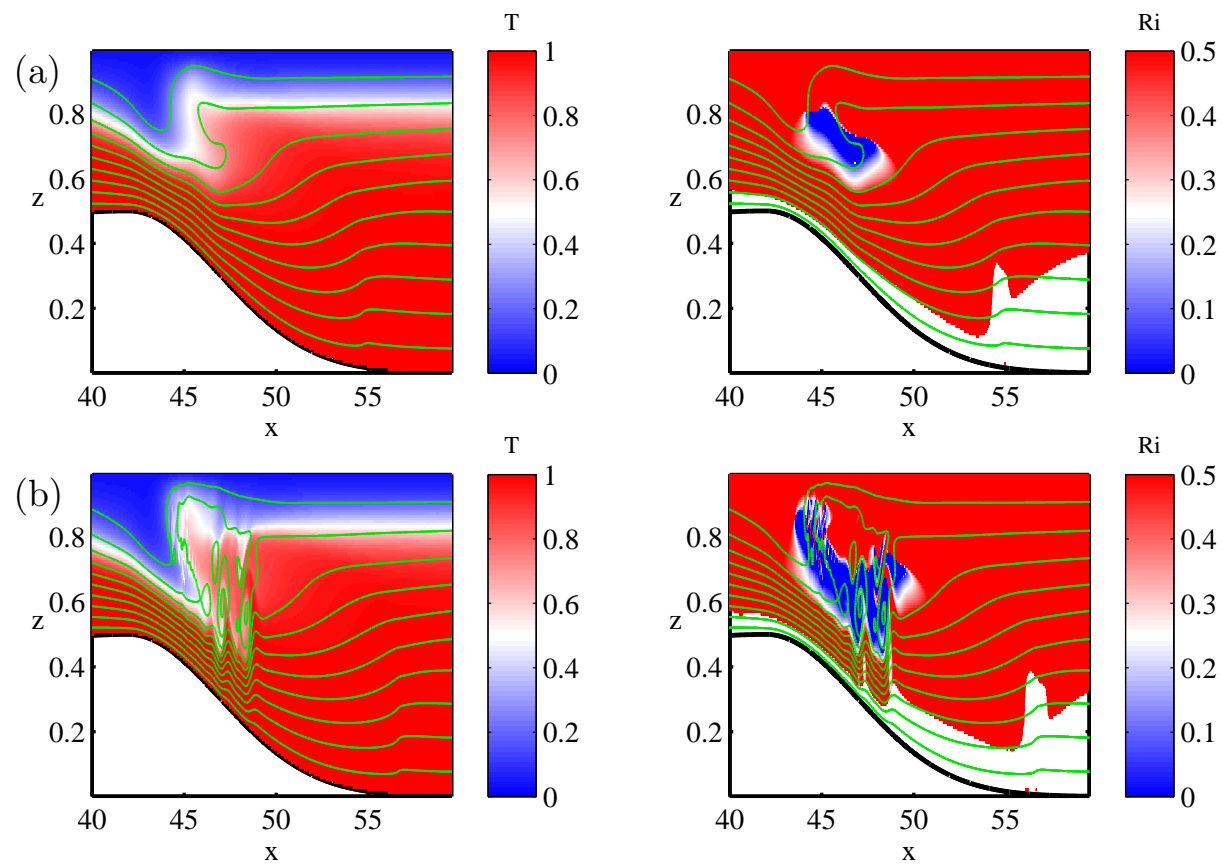

Figure 4-7: First-mode jump with breaking lee wave. $q=0.1, h_{T}=0.5, T_{T}=380$, two-layer stratification, and $\nu=7$ (defined in (4.3.1)). (a) $t=108$, (b) $t=124$. Green lines in plots of $R i$ are streamlines. 

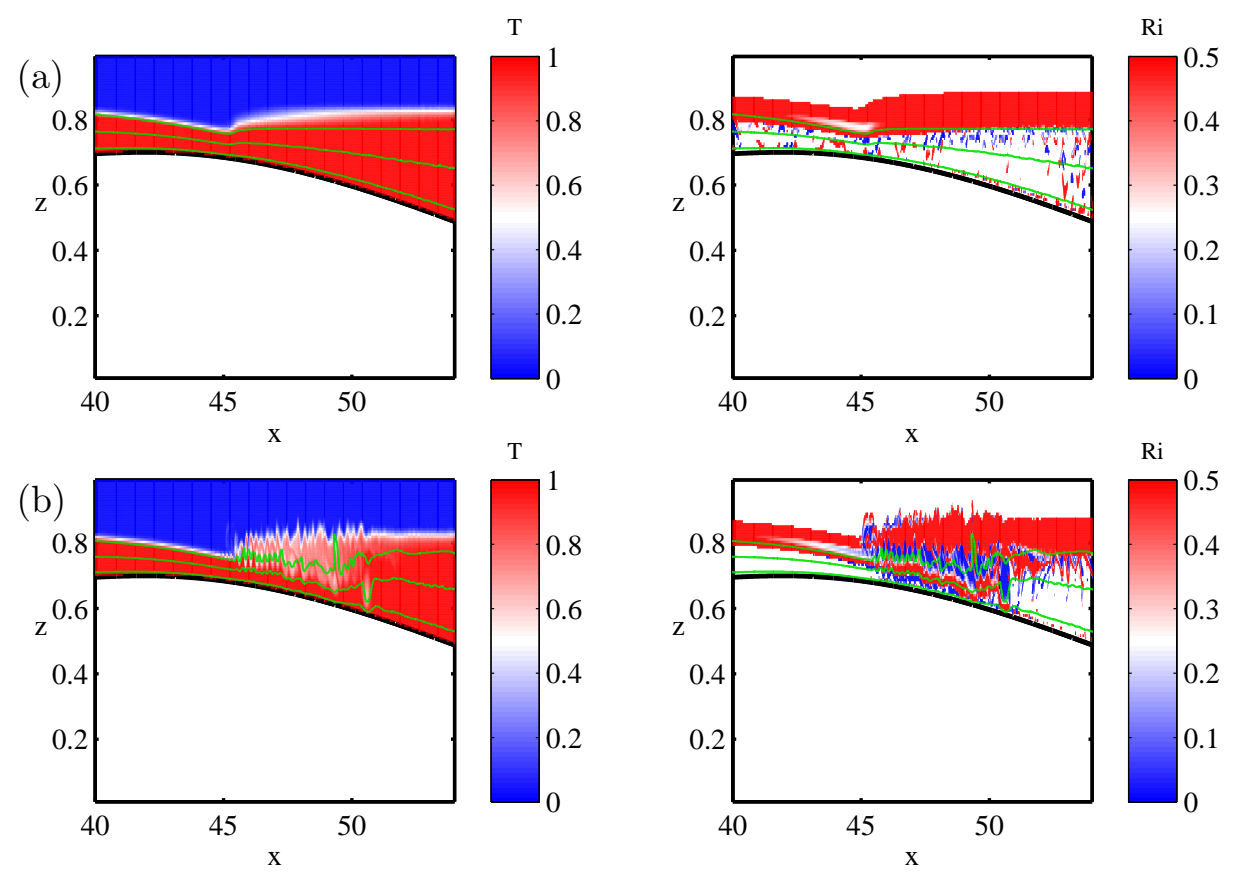

Figure 4-8: Higher-mode jump with upstream shear instability. $q=0.040, h_{T}=0.7$, $T_{T}=380$, two-layer stratification, and $\nu=20$ (defined in (4.3.1)). Upstream flow has a sharper interface with $\lambda=90, z_{0}=0.83$. (a) $t=140$, (b) $t=180$. Green lines in plots of $R i$ are streamlines.
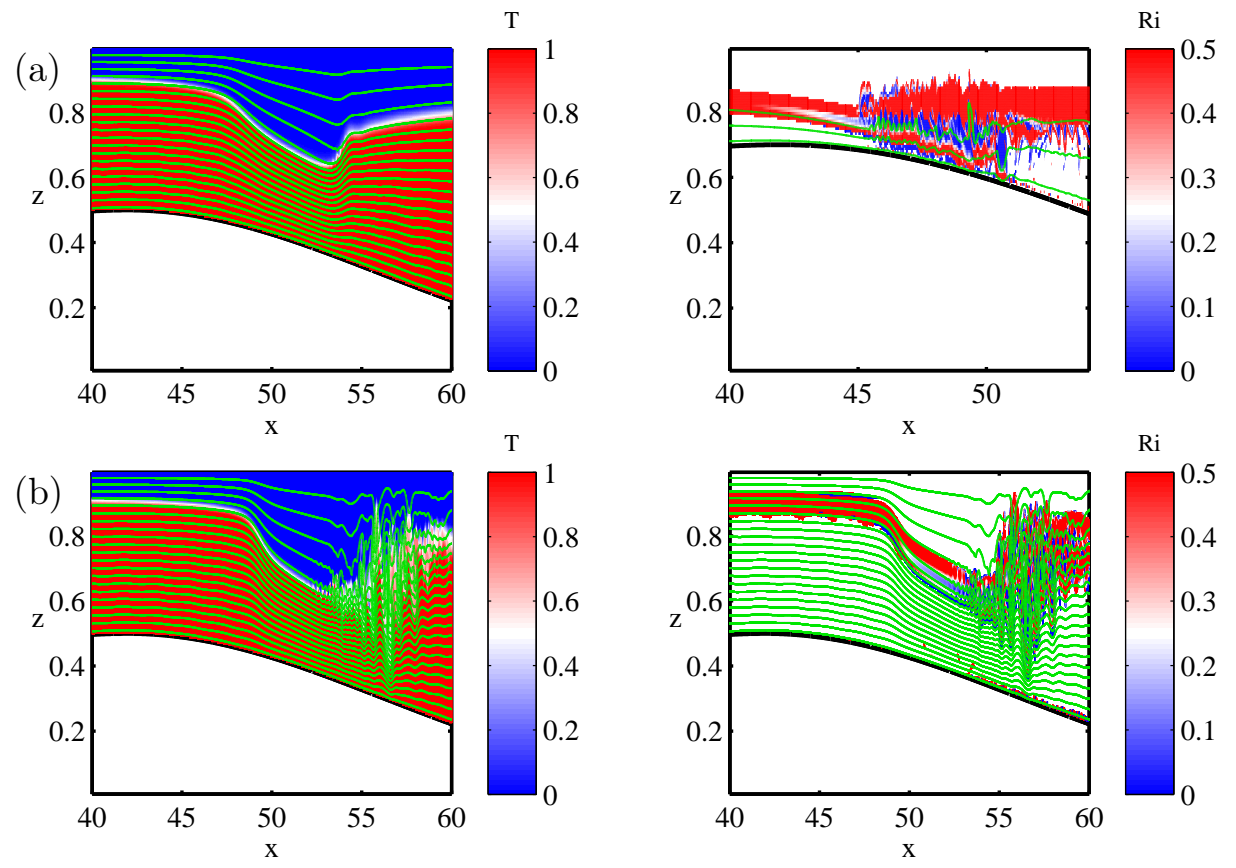

Figure 4-9: First-mode jump with upstream shear instability. $q=0.2, h_{T}=0.5, T_{T}=380$, two-layer stratification, and $\nu=20$ (defined in (4.3.1)). Upstream flow has a sharper interface, with $\lambda=90$. (a) $t=110$, (b) $t=128$. Green lines in plots of $R i$ are streamlines. 
stabilities occur in a higher-mode jump (examples are shown in figures 4-6 to 4-9). In jumps without upstream shear instabilities, whether the jump type is an overturning wave or a higher-mode jump with a wedge depends primarily on the volume flow rate and less so on the topographic height, as shown in figure $4-10\left(z_{0}=0.83\right.$ and $\left.\lambda=10\right)$. In these flows, the interface is too thick for shear instabilities to develop, so all of the jumps are overturning waves or higher-mode jumps with wedges. Note that this regime diagram includes simulations with different downstream slopes (mainly $\nu=7$, defined in (4.3.1), but some with $\nu=20$ and 50 are included) and tidal time periods ( $T_{T}=380$ for most simulations, and $T_{T}=1200$ for some simulations).

The stratification and downstream topographic slope are the most important parameters in determining whether upstream shear instabilities develop and have space to grow. The regime diagram in figure 4-11 shows the jump types that occur in $q-h_{T}$ space for $z_{0}=0.83, \nu=20$, and $\lambda=90$ (defined in (4.3.1)). With a thinner interface and gentler topographic slope, most jumps develop upstream shear instability. Low volume flow rates over lower topography can result in a undular bore with downstream shear instability. However, if $q$ is large enough, the jump smothers the upstream shear instability, resulting in a wave-overturning type jump. The background interface height has not been varied, although the same jump types are expected to form based on the results of chapter 2. However, the parameters values at which the jump type changes likely shift as the interface height changes. The upstream interface height does vary between simulations because it is determined by $q, h_{T}$, and the location of the jump.

Whether a wave-overturning or shear instability-type jump occurs depends primarily on the stratification and downstream slope, so while the $q-h_{T}$ position of Knight Inlet, Hood Canal, and Stellwagen Bank are indicated in figures 4-10 and 4-11, they each have unique stratifications and topographies that affect the actual qualitative jump type. Finally, figure 4-12 shows the jump types in $q-\nu$ space for $z_{0}=0.83$, $\lambda=90$, and $h_{T}=0.5$, indicating roughly the slope at which jumps transition to having upstream shear instability for flows with thin interfaces. 


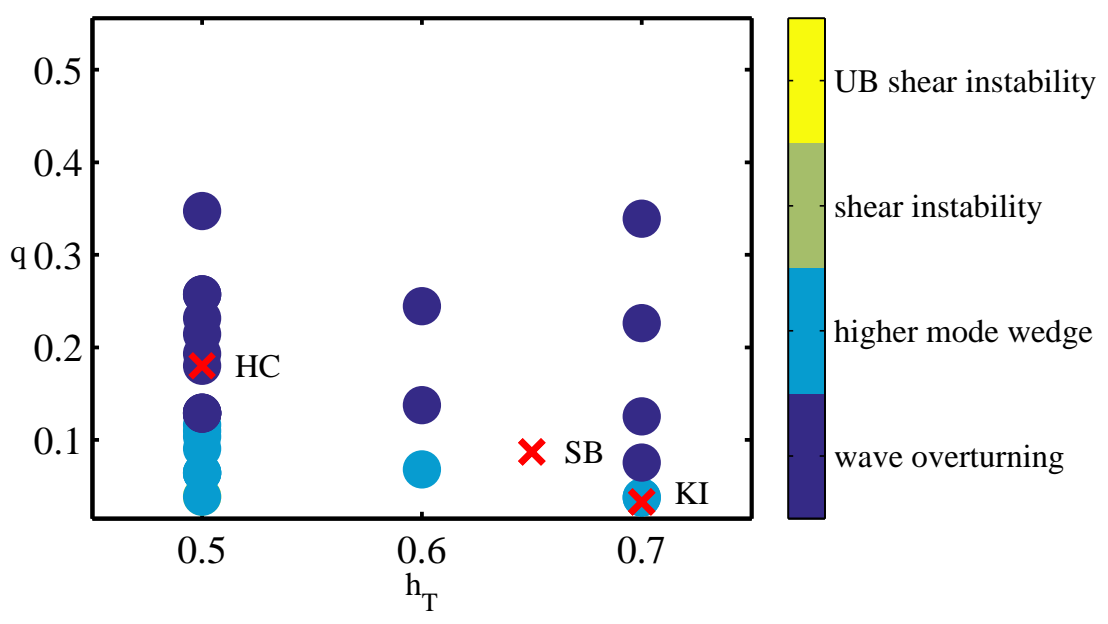

Figure 4-10: Regime diagram for flows with varying topography. $z_{0}=0.83, \nu=7 . q$ and $h_{T}$ observations of Knight Inlet (KI), Hood Canal (HC), and Stellwagen Bank (SB) are indicated by $\times$ in $q-h_{T}$ space, although they have different stratifications and topographic slopes.

The undular bore with trailing shear instabilities occurs at the transition between mode-one and higher-mode jumps with upstream shear instabilities. It is a mode-one jump, and occurs at the lowest forcing for which mode-one jumps develop, which is consistent with undular bores developing for low $U_{0}$ and low shear in chapter 2 . This is shown in figure 4-11, which shows that the undular bore occurs for low $q$ and low $h_{T}$, which results in low upstream shear. 


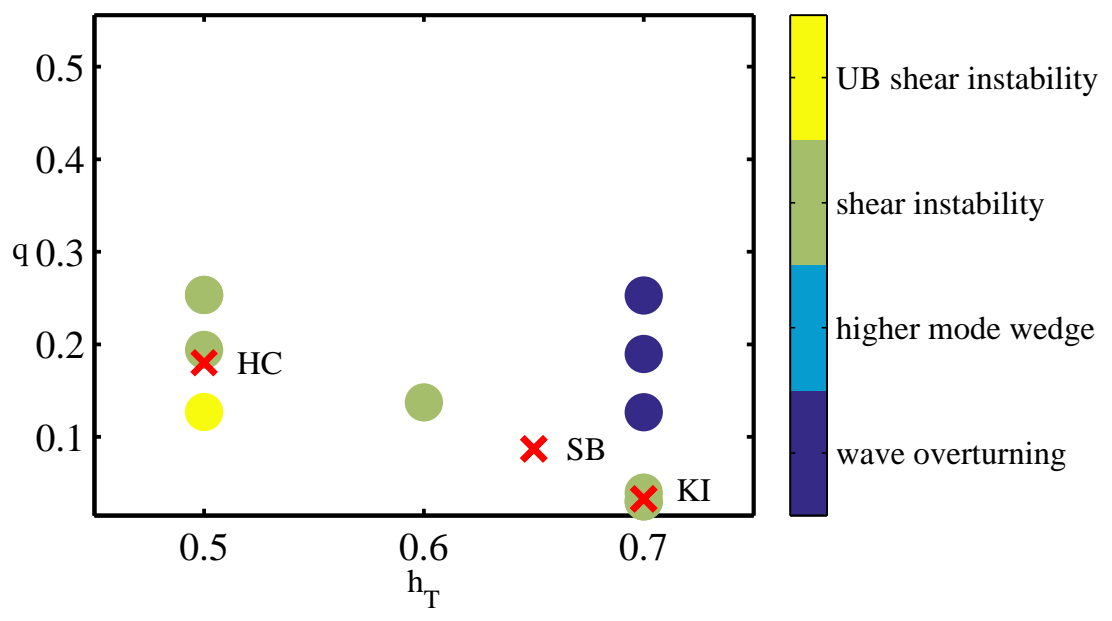

Figure 4-11: Regime diagram for flows with varying topography. $z_{0}=0.83, \nu=20, \lambda=90$. $q$ and $h_{T}$ observations of Knight Inlet (KI), Hood Canal (HC), and Stellwagen Bank (SB) are indicated by $\times$ in $q-h_{T}$ space, although they have different stratifications and topographic slopes.

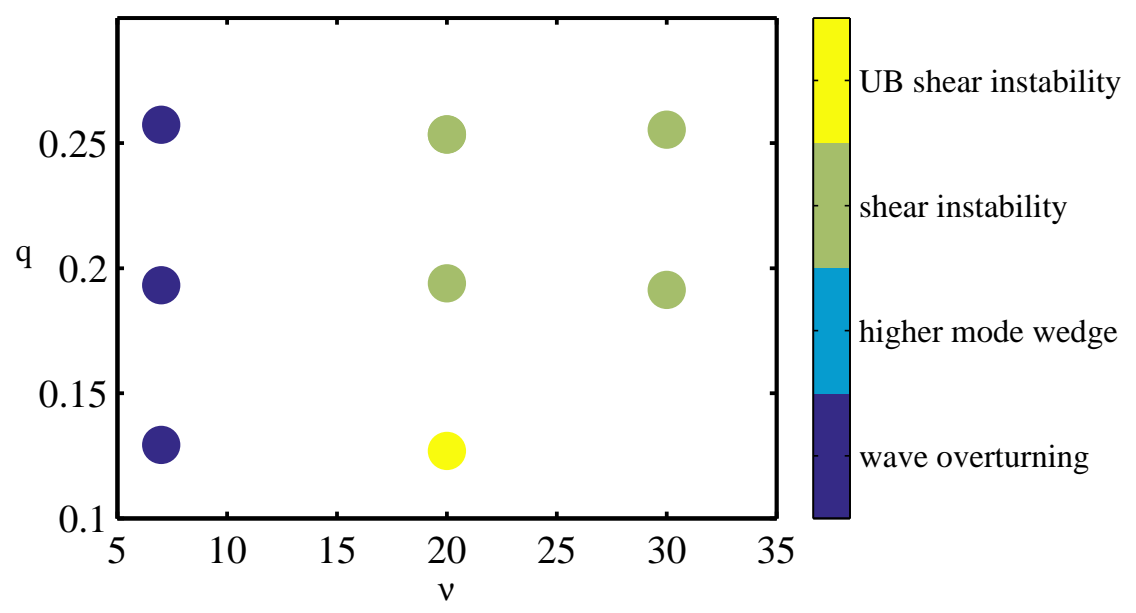

Figure 4-12: Regime diagram for flows with varying downstream slope. $z_{0}=0.83, \lambda=90$, $h_{T}=0.5$. 


\section{Wedge}

Wedge regions of stangnant, homogeneous fluid of intermediate density have previously been described, particularly in the context of Knight Inlet. Armi and Farmer (2002) look at the wedge with a variety of forcing and mention the influence of topographic slope, focusing on Knight Inlet. This work considers a wider range of parameter values to include the type of flow that occurs in other locations (Hood Canal, Knight Inlet, the pre-Bosphorus, and Stellwagen Bank) to assess under what conditions a wedge will form, and to analyze the characteristics of this type of jump. Identifying when a wedge will form is important because it can decouple the layers and allow the jump to be treated as single layer with reduced gravity (Farmer and Armi, 1999; Winters and Armi, 2012). Alternatively, a three-layer approximation could more accurately describe the jump where the wedge is the third layer in between the original two layers.

The wedge is identified as a region of approximately homogeneous, stagnant fluid just ahead of the jump. The isopycnals and streamlines should diverge in the region of the wedge, with some plunging down over the topography while upper streamlines deflect upwards, forming the wedge between. Based on the simuation results discussed above, the wedge appears to be a feature of higher-mode jumps, and occurs for smaller forcing $\left(0.03 \lesssim q \lesssim 0.09\right.$ with $0.5 \lesssim h_{T} \lesssim 0.7$, see figure $4-6$ and $\left.4-10\right)$. It is classified as a higher-mode jump based on the long wave phase speeds calculated from the continuous density and velocity profiles of the entire depth of the flow. As a higher-mode jump, it is also transient, as lower-mode disturbances move both upstream and downstream of the jump and alter the flow in both directions. The transient development, however, is slow; the jump is quasi-steady, and can be analyzed by averaging over a time that is short relative to the timescale of the development. In a higher mode internal jump, the diverging streamlines or isopycnals divide the flow into approximately three layers: the lower, active layer, the middle layer containing the wedge, and the upper layer. Due to this division, the two-layer approximation used in chapters 2 and 3 is particularly innappropriate. The single layer approach of Farmer and Armi (1999) might provide an accurate estimate of the 
jump height. In this approach, the active layer is treated as a single layer flow, with reduced gravity based on the density of the stagnant wedge region. In this case, the criticality of the flow is based only on the lower layer, and not on a continuous profile, and therefore the flow may be classified as super-critical. However, a three layer or continuous model would provide a better description of the entire depth of the flow.

Higher mode jumps in the environment appear to exhibit a wedge region, as seen in Knight Inlet (figure 1-2) and the Pre-Bosphorus Channel (figure 1-5), which is an exchange flow with continuous stratification and would therefore allow waves to travel upstream of the jump (§3.3.2).

\section{Shear instability versus wave breaking}

There has been significant discussion in the literature about whether shear instabilities or wave overturning cause the mixing associated with a hydraulic jump. The discussion is often focused on Knight Inlet, which has been well-studied both observationally and numerically. In that context, Armi and Farmer (2002) argue that "Small-scale shear instabilities are responsible for the initial phase of mixing above the obstacle crest," and are the source of the mixed fluid in the wedge. When analyzing the flow in Knight Inlet, they note that without bottom boundary layer separation, wave overturning will occur instead. Lamb (2004) and Afanasyev and Peltier (2001), however, find a breaking lee wave causes the mixing and formation of a stagnant mixed layer. Lamb (2004) studied the importance of bottom friction and bottom boundary layer separation and concluded that they are important to the flow structure, but that wave overturning is still the main cause of mixing in Knight Inlet.

The simulations shown so far do not include bottom friction, which is required for bottom boundary layer separation. However, Cummins (2000) simulates bottom boundary layer separation with modified topography. Therefore, the simulations described here are generally relevant if the topography used in the simulation is considered 'modified'. The modified topography approach was supported by Farmer and 
Armi (2001). However, the flow with modified topography is not equivalent to a flow with bottom friction and boundary layer separation; for example, shear instabilities on the underside of the separated jet (Winters and Armi, 2013) will not occur if modified topography is used instead of bottom friction. Nonetheless, the modified topography can create a flow in which the dense jet of fluid spilling over the topography takes approximately the same path it would take if bottom boundary layer separation occured at the same location. Regardless, simulations with viscosity near the bottom, described later in $§ 4.3 .6$, show that bottom viscosity and a no-slip bottom boundary condition can cause a flow to develop upstream shear instabilities.

Doyle et al. (2005) studied large-amplitude lee waves breaking over mountains in Greenland, obtaining detailed field observations and numerically simulating the observed event. Breaking lee waves seem to be more prevelant in the atmosphere, while shear instability-driven mixing may occur in channels such as Knight Inlet. This is likely due to the stratification, downstream topographic slope, and an effectively infinitely deep atmosphere. Winters and Armi (2013) conduct general simulations of uniformly stratified flow and again claim that the mixing is caused by shear instability rather than overturning lee waves in flows with low frequency forcing (quasi-steady flow) and low topographic Froude number $\left(\frac{U}{N h_{T}}\right.$, where $U$ is the barotropic forcing velocity away from the topography, $N$ is the buoyancy frequency, and $h_{T}$ is the topographic height). However, they find the highest shear occurs on the underside of the separated jet, rather than along an upstream interface.

The simulations shown here indicate that mixing can be initiated by either overturning waves or shear instability, depending on the parameter regime. All simulations have an approximately two-layered flow with a constant interface depth. The most influential parameters in determining whether wave overturning or shear instability initiates the mixing appear to be the interface thickness and the slope of the downstream topography. For shear instability to develop, the interface must be thin enough for the flow to become unstable and the topographic slope must be gentle enough to allow space for the perturbation to grow. Jumps with upstream shear 
instability may be mode-one or higher-mode jumps, and therefore exist for a wide range of volume flow rates. Flow separation could also provide for an effectively gentler slope and allow upstream shear instabilities to develop (Farmer and Armi, 2001), as investigated later in $§ 4.3 .6$. Flow separation also allows shear instabilities to develop on the underside of the separated jet, as seen by Winters and Armi (2013), and illustrated later in $§ 4.3 .6$.

The qualitative jump types and conditions under which each occurs are consistent in $3 \mathrm{D}$, as described in $\S 4.3 .7$.

\subsubsection{Mass budgets}

Mass budgets are used to identify the source of intermediate density water in the jump region. This is of particular interest for higher-mode jumps that develop a wedge of stagnant, nearly homogeneous water of intermediate density because the mass budget clarifies the source of the water in the wedge. However, even for the other jump types, mass budgets will be used here to indicate characteristics such as the magnitude of mixing compared to advection.

The fluid is divided into three water masses based on density. Water mass $M_{1}$ is dense water with $T>0.7$, water mass $M_{2}$ is intermediate water with $0.3 \leq T \leq$ 0.7 , and water mass $M_{3}$ is light water with $T<0.3$. The mass budgets are not highly sensitive to these bounds, and the values were chosen so that the intermediate water only existed in a thin layer upstream of the jump but filled a thicker layer downstream of the jump. $M_{i}(t)$ is the total volume of the $i^{\text {th }}$ water mass at time $t$. The change in volume of each water mass, $\Delta M_{i}(t)=M_{i}(t)-M_{i}(0)$ is calculated, and the contributions of net advection into the domain and mixing are also determined. The contribution from the divergence of advection is given by

$$
M_{a d v, 1}(t)=\int_{0}^{t}\left[\int_{0}^{D} u H(T-0.7) d z\right]_{b}^{a} d t^{\prime}
$$




$$
\begin{gathered}
M_{a d v, 2}(t)=\int_{0}^{t}\left[\int_{0}^{D} u H(0.7-T) H(T-0.3) d z\right]_{b}^{a} d t^{\prime} \\
M_{a d v, 3}(t)=\int_{0}^{t}\left[\int_{0}^{D} u H(0.3-T) d z\right]_{b}^{a} d t^{\prime} .
\end{gathered}
$$

for each water mass, where $H(\cdot)$ is the Heaviside function. The contribution from mixing is the residual, given by $M_{m i x, i}(t)=\Delta M_{i}(t)-M_{a d v, i}(t)$.

The water mass budgets of four simulations, including a higher-mode jump with a wedge, a first-mode jump with wave overturning, a first-mode with shear instabilities, and a higher mode jump with shear instabilities are shown in figures 4-13 and 4-14. The mass budgets for the wedge (figure 4-13) and first mode jump with upstream shear instability (figure 4-14) are for the simulations depicted in figures 4-6 and 4-9. A different jump with wave overturning is shown in figure 4-14 than in figure 4-7 so that the volume flow rate of both mode-one jumps in figure 4-14 are equal. A different higher-mode jump with upstream shear instability is shown in figure 413 to better illustrate the initial contribution of advection. Figures 4-13 and 4-14 shows $\Delta M_{i}(t)=M_{i}(t)-M_{i}(0)$ for each water mass in the upper panel. The second (third/fourth) panel from the top shows $\Delta M_{i}(t)$ of the light (intermediate/dense) water mass and the contributions from the divergence of advection and mixiing.

The water mass budget shown in the left column of figure 4-13 identifies the source of the intermediate density fluid in the wedge region (third row) of the higher-mode jump seen in figure 4-6 as mainly net advection in from upstream. The streamlines at the interface spread in the region of the jump, as seen in figure 4-6, and water advects in from upstream, creating a pocket of fluid of intermediate density that remains within the jump region. Mixing does not play a significant role in the mass balance in these higher-mode jumps, although some mixing does occur, as seen in figure 4-6. The wedge region initially grows as intermediate density fluid advects in from upstream, but the volume of intermediate water reaches an approximately steady value as the jump becomes fully developed. As a higher-mode jump, it continues to change slowly with time, although it does reach a quasi-steady state after the forcing reaches 
the maximum value.

In first mode jumps with wave overturning (figure 4-14, left column), net advection initially has a significant contribution to the intermediate mass budget. At a time of about $t \approx 70$, the flow enters a regime of more intense mixing as shear instabilities develop along edges of the overturning wave. This mixes the light and dense waters, and mixing becomes the most significant source of intermediate water. When this highly mixed region reaches the downstream boundary $(t \approx 110)$, the net advection of intermediate water becomes out of the domain, so the cumulative contribution of advection $\left(M_{a d v, 2}\right)$ begins to decrease, and soon after becomes negative. Once the mixed region reaches the downstream boundary, mixing and advection balance, and the amount of intermediate water becomes steady with time. The time at which this occurs depends on the extent of the calculation domain, which ends at $x \approx 60$ for the mass budgets shown. Timing of these events also depends on the tidal period, which is $T_{T}=380$ for all of the results shown in figures $4-13$ and $4-14$. The higher-mode wedge (figure 4-6 and left column of figure 4-13) does not experience this intense mixing, and mass flux divergence divergence remains the primary contributor to the intermediate water mass, which comprises the wedge.

First-mode jumps with upstream shear instability (figure 4-14, right column) are similar to jumps with wave overturning in that net advection is initially important to the intermediate water mass budget. As shear instabilities develop, mixing takes over as the main source of intermediate water, and shortly after, there is a net advection out as the intermediate water that is produced by mixing advects out of the jump region. The mass budgets suggest that the total mixing is similar in jumps with and without upstream shear instability.

Higher-mode jumps with upstream shear (figure 4-13, right column) generate intermediate water through similar contributions of advection and mixing during the early stages of the jump development, but mixing takes over as the main source of intermediate fluid in the later stages of jump development as shear instabilities at 
the interface grow. Although these are also higher mode jumps, the mass budget is different from the wedge-type jumps because mixing plays an important role in the intermediate mass budget, while advection is the most significant contributor of intermediate water in jumps with wedges.

Note that mixing is very similar between the cases with wave overturning and shear instability. In the case with shear instability, the mixing is delayed due to the gentler topographic slope, but the mixing and advective terms make similar contributions to the mass budgets of the two jump types. However, the amount of intermediate water that is produced is much greater in the simulation with wave overturning. This is likely a result of the geometry of the domain; while both mass budgets are calculated using the same domain length, the flow with wave overturning has steeper topography, and therefore a greater water depth away from the sill crest, resulting in a larger domain and more space for intermediate water, so more is produced.

The ratio $\frac{M_{a d v, 2}}{\Delta M_{2}}$ versus $q$ for jumps without upstream shear instabilities is shown in figure $4-15$ a. For jumps with upstream shear instabilities, $\frac{M_{a d v, 2}}{\Delta M_{2}}$ versus $q$ is shown in figure 4-15b. All jump types are shown together in figure 4-15c. Curves of best fit are also shown. The figure shows a single value for each simulation, which is obtained by averaging the ratio through a region in time; example regions are indicated by shading in figures 4-13 and 4-14. The averaging region is chosen as a short interval after $\Delta M_{2}$ reaches an approximately constant value. This is a subjective choice, and other valid approaches could be taken instead. The approach was chosen because when $\Delta M_{2}$ becomes constant in time, the jump has just reached a quasi-steady state. However, $M_{a d v}$ and $M_{m i x}$ will continue to change in time, for example if intermediate fluid is continuously generated within the domain and then advected out. While the tidal period $T_{T}$ affects the timing of events such as the onset of mixing, it does not effect the ratio $\frac{M_{a d v, 2}}{\Delta M_{2}}$, and the figure includes many simulations with $T_{T}=380$ and some with $T_{T}=1200$.

Without upstream shear instabilities, the ratio drops from $\frac{M_{a d v, 2}}{\Delta M_{2}} \approx 1$ to $\frac{M_{a d v, 2}}{\Delta M_{2}} \lesssim 0$ 
wedge
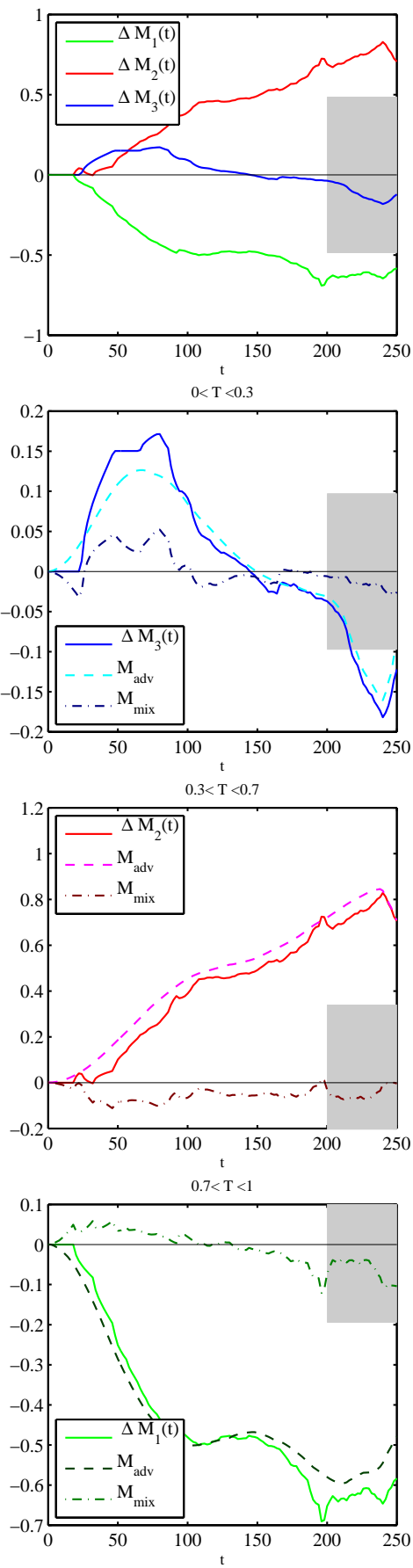

higher-mode shear
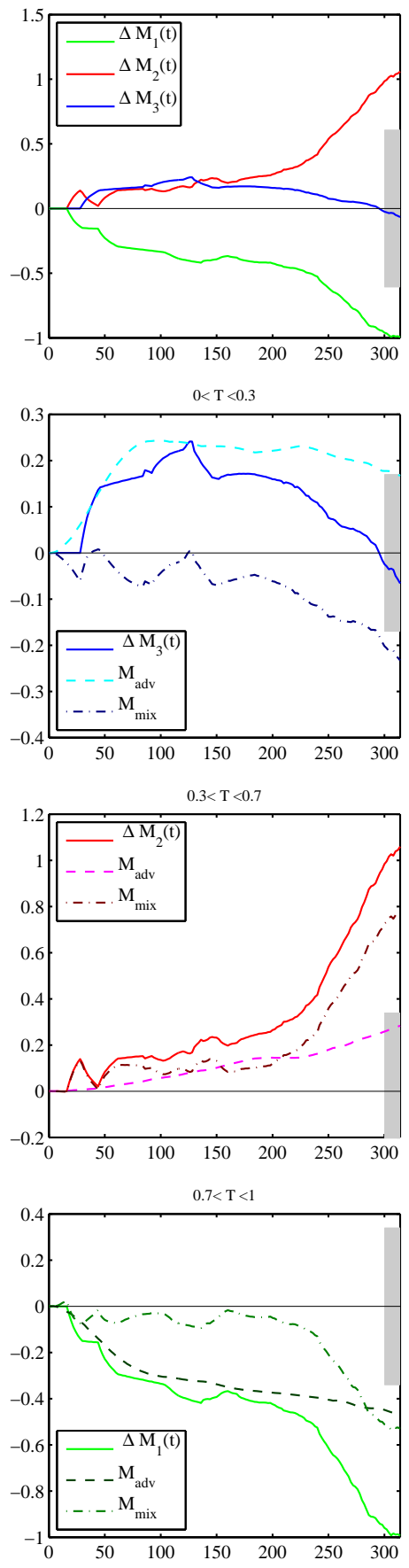

Figure 4-13: Water mass development with time. $\Delta M_{i}(t)=M_{i}(t)-M_{i}(0)$ for each water mass (top). Second (third/fourth) panel from the top shows $\Delta M_{i}(t)$ of the light (intermediate/dense) water mass and the contributions from the divergence of advection and mixing. Higher mode jump with wedge $(q=0.065, \lambda=10, \nu=7)$ (left, shown in figure 4-6) and higher-mode jump with shear instability $(q=0.030, \lambda=90, \nu=20$, different figure 4-8 to more clearly illustrate initially equal contributions of advection and mixing). $T=380$, $h_{T}=0.5$. The shaded gray region indicates the region over which $\frac{M_{a d v}}{\Delta M}$ is averaged to calculate the values used in figure 4-15. The region is chosen where $\Delta M_{2}$ becomes approximately constant. 

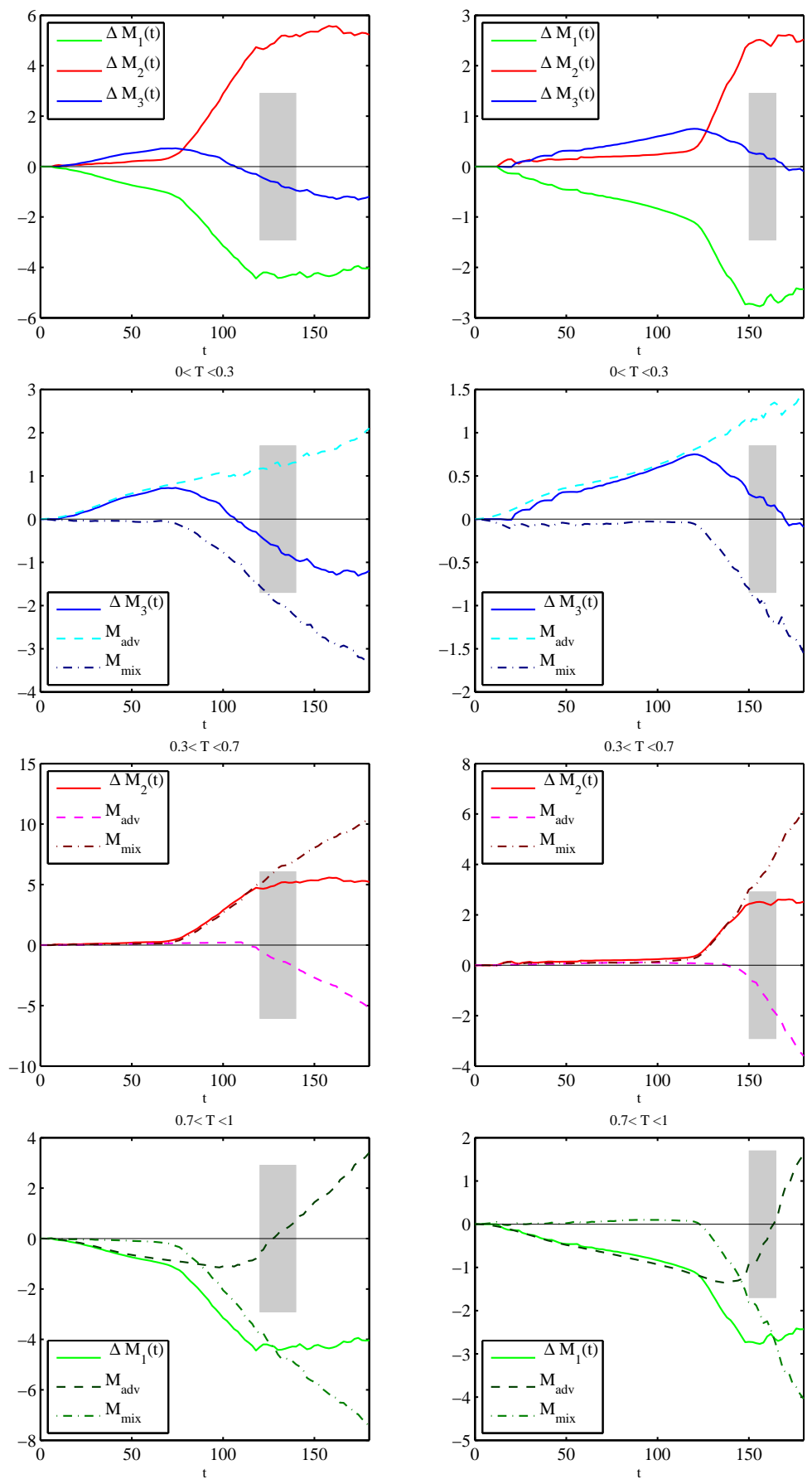

Figure 4-14: Water mass development with time. $\Delta M_{i}(t)=M_{i}(t)-M_{i}(0)$ for each water mass (top). Second (third/fourth) panel from the top shows $\Delta M_{i}(t)$ of the light (intermediate/dense) water mass and the contributions from the divergence of advection and mixing. First-mode jump with wave overturning $(q=0.25, \lambda=90, \nu=7$, different from figure 4-7 so that volume flow rate matches first mode jump with shear instability) (left) and first mode jump with shear instability $(q=0.25, \lambda=90, \nu=20$, shown in figure 4-9) (right, different from figure $4-7) . T=380, h_{T}=0.5$. The shaded gray region indicates the region over which $\frac{M_{a d v}}{\Delta M}$ is averaged to calculate the values used in figure $4-15$. The region is chosen where $\Delta M_{2}$ becomes approximately constant. 
as $q$ increases. This indicates that as the volume flow rate increases, the contribution of advection to the intermediate water mass decreases from being the main contributor to being negligible as mixing takes over. Negative values of $\frac{M_{a d v, 2}}{\Delta M_{2}}$ indicate that intermediate water is being produced by mixing and then advected out. Figure 415a only includes higher-mode jumps with wedges and first-mode jumps with wave overturning for which the interface is thick enough that upstream shear instability does not occur. The figure shows that there is a continuous transition from advection being the exclusive producer of intermediate water to mixing being the main source, rather than an abrupt transition when the jump type changes from a higher-mode jump with a wedge to a jump with wave overturning. There is, however, an abrupt transition between jump types because they are categorized into discreet types.

When upstream shear instabilities occur, mixing contributes more significantly to the production of intermediate water, even at low volume flow rates (figure 4-15, top right). As the volume flow rate increases, the contribution for mixing increases further, until mixing is equally important in jumps with and without upstream shear instabilities (figure 4-15, bottom). 

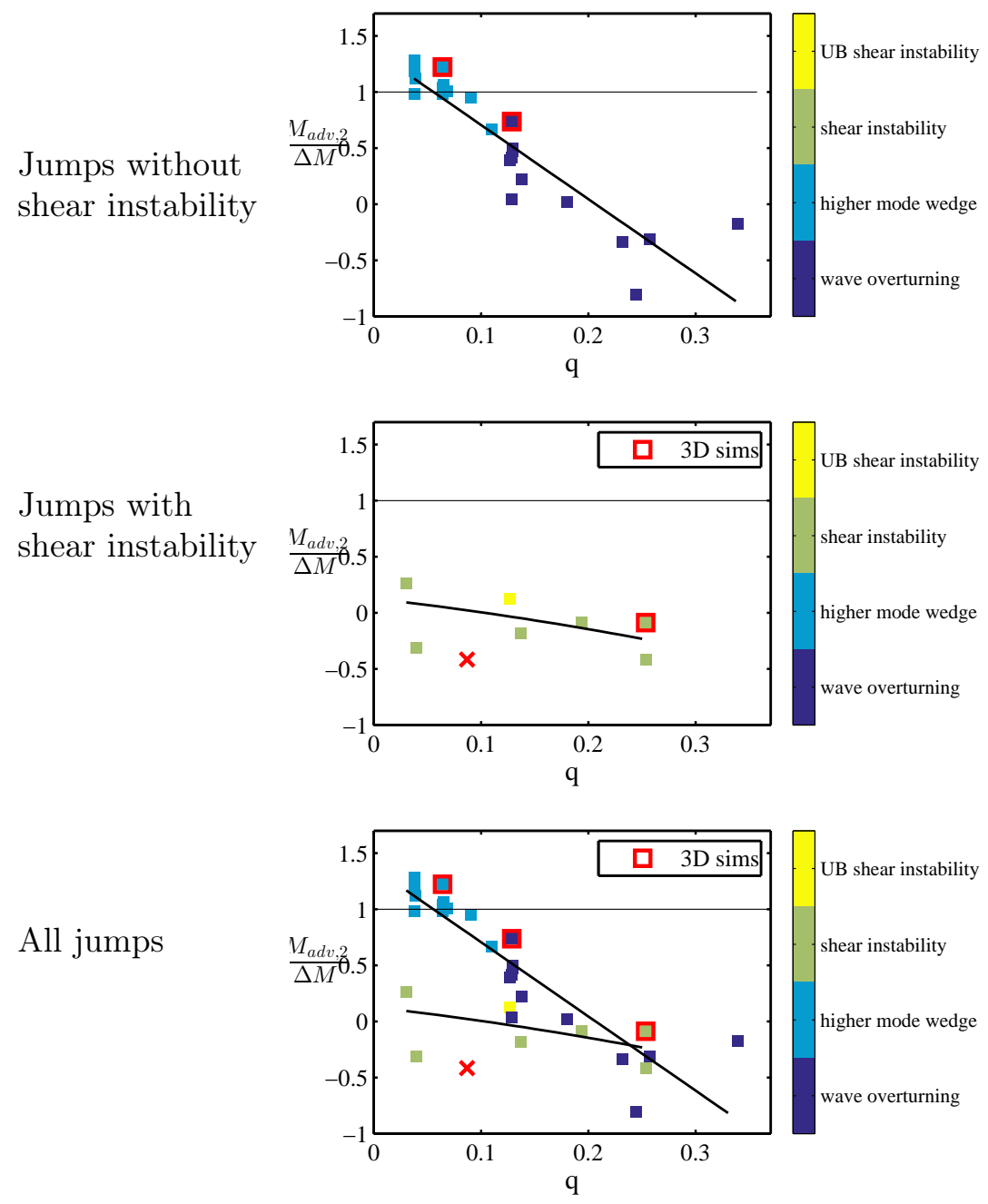

Figure 4-15: $\frac{M_{a d v, 2}}{\Delta M_{2}}$ for the intermediate fluid $(0.3<T<0.7)$. Jumps without upstream shear instabilities are shown in the top panel. Jumps with upstream shear instabilities are shown in the second panel. All jumps are plotted together in the bottom panel. Stellwagen bank simulation result (see $\S 4.4$ ) is indicated by $\times$. 3D simulation results (see $\S 4.3 .7$ ) are indicated by $\square$. 


\subsubsection{Mixing}

The mixing efficiency, $R i_{f}$ (2.4.4), is plotted versus volume flow rate $q$ for the a set of 2D simulations (and two 3D simulations) in figure 4-16. Almost all of the 2D simulations have a mixing efficiency $R i_{f} \approx 0.5$, which is consistent with the $2 \mathrm{D}$ low shear jumps described in chapter 2. However, a few of the simulations have uninformative values that are much lower, or even negative. An example is shown in figure 4-17, which depicts the cumulative integrals of the turbulent shear production (2.4.3), buoyancy flux (2.4.2), and mixing efficiency (2.4.4) along the domain, as well as the time-averaged tracer field, of a simulation with $q=0.076, T_{T}=380, h_{T}=$ $0.7, \nu=7, \lambda=10$ (defined in (4.3.1)), $R i_{f}=-0.44$. In this case, there is upstream adjustment ahead of the point where the flow transitions to super-critical, resulting in a negative cumulative buoyancy flux $(M)$, and this affects the cumulative integral of the mixing efficiency ahead of the jump. The integral could be calculated starting from a point further downstream, or this datum could be discarded. Lower $R i_{f}$ values may also occur for sub-critical flows because disturbances can progagate upstream of the jump, disturbing the flow at the beginning of the integrated region. By restarting the cumulative integral at the point of the minimum cumulative buoyancy flux as shown in figure 4-17 $(x \approx 45)$, the effect of upstream adjustment is eliminated and the resulting value of $R i_{f}$ is reasonable. Applying this approach to all of the simulations with very small or negative $R i_{f}$ in figure $4-16$ and considering only $2 \mathrm{D}$ mode-one jumps gives the distribution of $R i_{f}$ shown in figure 4-18. With this adjustment, the $2 \mathrm{D}$ mixing efficiencies are all approximately 0.5 , which is consistent with the efficiencies found in chapter 2 . To avoid the sensitivity of $R i_{f}$ on the domain, turbulence quantities based on a width average (for 3D simulations) or an ensemble average is many simulations that are equivalent except for the addition of some random background noise are conducted. A spatial average has been applied for calculating mixing efficiencies by Itsweire et al. (1993), for example. A similar difficulty with a slowly varying flow making averging over long times a problem was encountered by Rohr et al. (1988), who were unable to reliably calculate the crossmoments that are required to calculate buoyancy flux and shear production. 


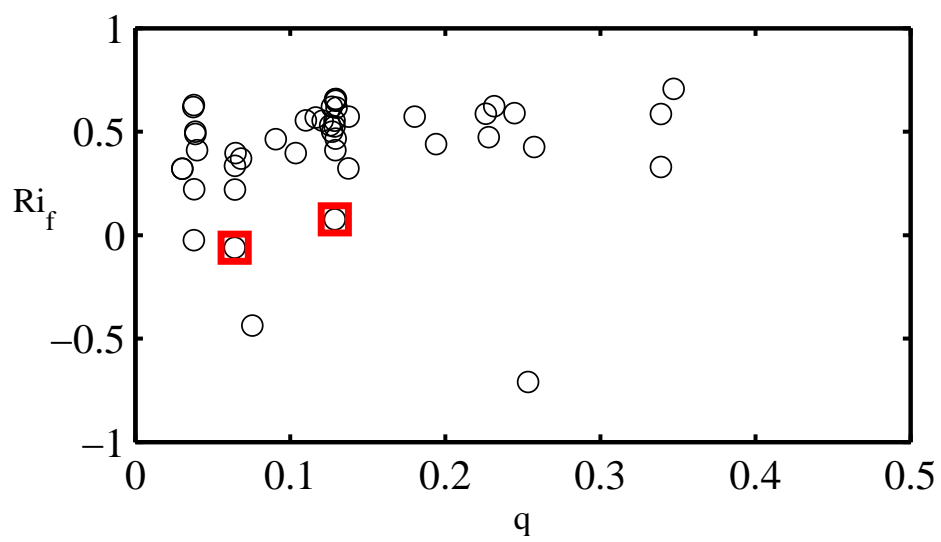

Figure 4-16: $R i_{f}$ for jumps with topography. 3D simulation results (see §4.3.7) are indicated by $\square$.

The mixing efficiencies for these simulations with topography are generally consistent with the results from $\S 2.4$. However, the efficiency does not vary between simulations and therefore does not indicate how much mixing occurs. To quantify the amount of mixing, a quantity such as the shear production of turbulent kinetic energy or the turbulent buoyancy flux on its own could be considered. Instead, the turbulent scalar variance equation is considered here. While the turbulent kinetic energy equation (2.4.1) includes buoyancy flux and shear production terms, as well as time variation, dissipation $(\epsilon)$, and transport terms, the turbulent scalar variance equation (Wyngaard and Coté, 1971),

$$
\frac{1}{2} \frac{\partial}{\partial t} \overline{\theta^{2}}+\frac{U_{i}}{2} \frac{\partial}{\partial x_{j}} \overline{\theta^{2}}+\overline{u_{j} \theta} \frac{\partial \Theta}{\partial x_{j}}+\frac{1}{2} \frac{\partial}{\partial x_{i}} \overline{u_{i} \theta^{2}}+D \overline{\frac{\partial \theta}{\partial x_{i}} \frac{\partial \theta}{\partial x_{i}}}=0
$$

only includes one term that can produce scalar variance, in addition to the time variation, dissipation $(\chi)$, and transport terms. In these equations, $\theta$ is the turbulent component of the scalar (in this case, scaled density), $u$ is the turbulence velocity, and capitals represent the mean values $\left(u_{t o t a l}=U+u\right)$. Because the scalar (scaled density) variance equation only includes one production term and dissipation (in addition to transport and time variation), the scalar production term is perhaps a better measure of the amount of mixing that occurs in the flow. Scalar (in this case density) variance production has been considered as a measure of turbulence by Wyngaard and Coté (1971), Shaw et al. (2001), and Burchard and Rennau (2008). The cummulative 

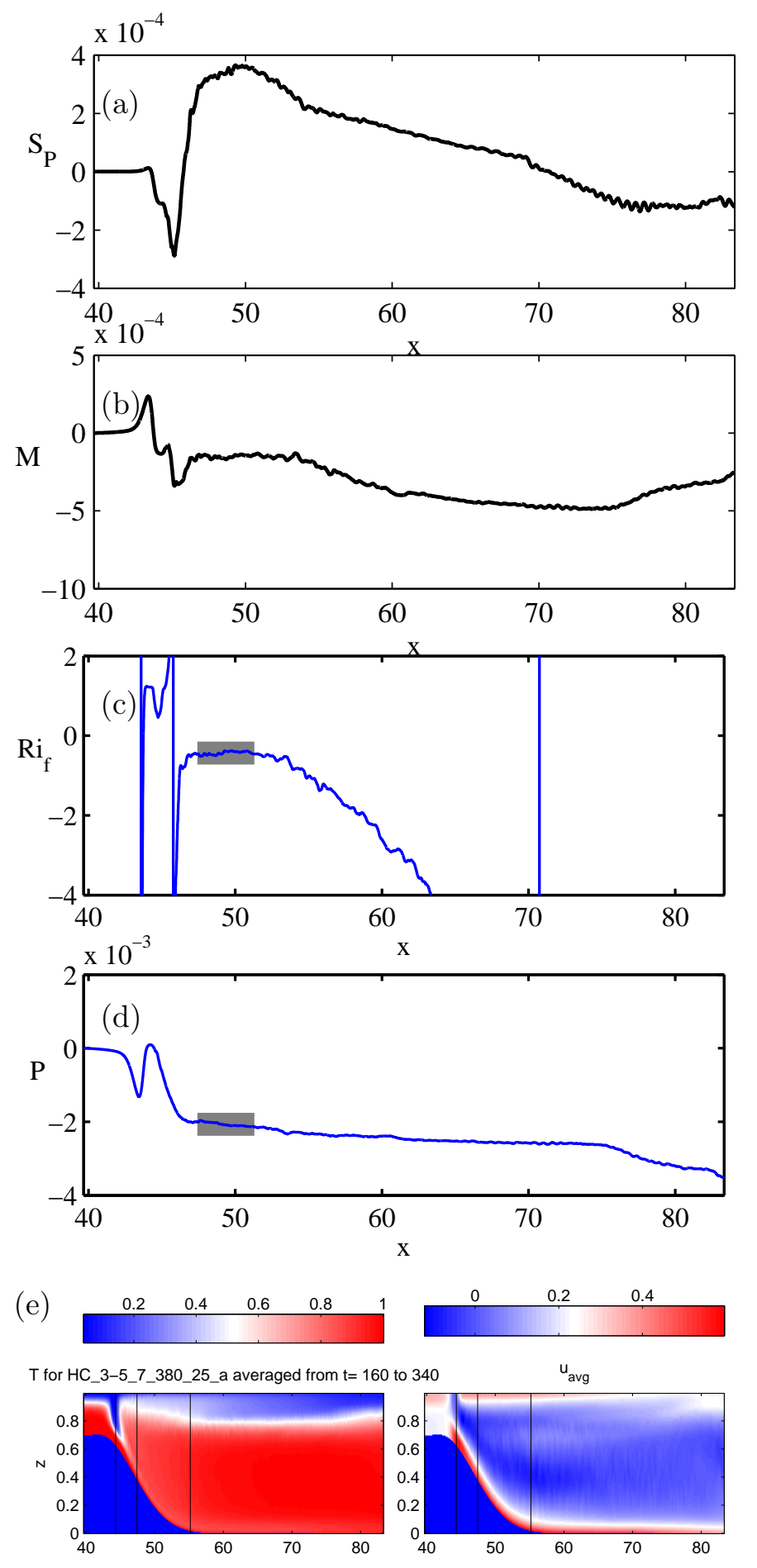

Figure 4-17: Cumulative integrals of (a) shear production of turbulent kinetic energy, (b) turbulent buoyancy flux, (c) Mixing efficiency, and (d) scalar variance production for a higher-mode jump with wedge $\left(q=0.076, T_{T}=380, h_{T}=0.7, \nu=7, \lambda=10, P=\right.$ $-0.0021, R i_{f}=-0.44$ ). (e) Time-averaged scaled density field $T$ (left) and horizontal velocity field ( $u$, right) averaged from $t=160$ to $t=340$, with data every $\Delta t=2$. 


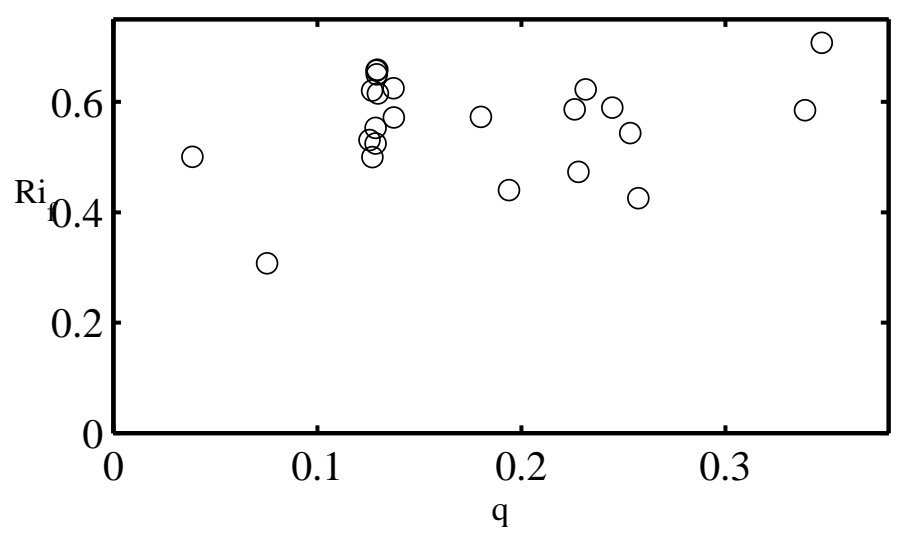

Figure 4-18: $R i_{f}$ for jumps with topography, corrected to account for upstream adjustment. 2D mode-one jumps are shown.

integral of the scalar variance production,

$$
P=\int_{a}^{x} \int_{h_{b}}^{H} \overline{u_{j} T} \frac{\partial T}{\partial u_{j}} d z d x
$$

is a measure of the total mixing that occurs in the jump. The cumulative intergral of the scalar variance production is also shown in figure 4-17. In this case, despite the problems with $R i_{f}, P$ asymptotes to an approximately constant value, $P=-0.0021$.

Another example of the cumulative integral of the density variance production is shown in figure 4-19. In this case, the upstream flow does not vary with time, so the mixing efficiency calculated from the cumulative integral produces a reasonable value. The simulation has a volume flow rate of $q=0.13, T_{T}=380, h_{T}=0.5, \nu=7, \lambda=$ 10, $P=-0.0032, R i_{f}=0.65, \frac{M_{a d v, 2}}{\Delta M_{2}}=0.48$. It is averaged from $t=160$ to $t=300$, with results saved at intervals of $\Delta t=2$. The jump is a mode-one internal hydraulic jump. The mixing efficiency is $R i_{f}=0.65$ and the scalar variance production is $P=-0.0032$, which indicates more mixing occurs than in the simulation depicted in figure 4-17, for which the scalar variance production is $P=-0.0021$.

The cumulative integral of the scalar variance production $P$ is mainly a function of shear, although shear and volume flow rate $q$ are also closely related. This is shown in figure 4-20, in which $P$ is plotted against $s, q, T_{T}, \nu, h_{T}$, and the mode of the jump 

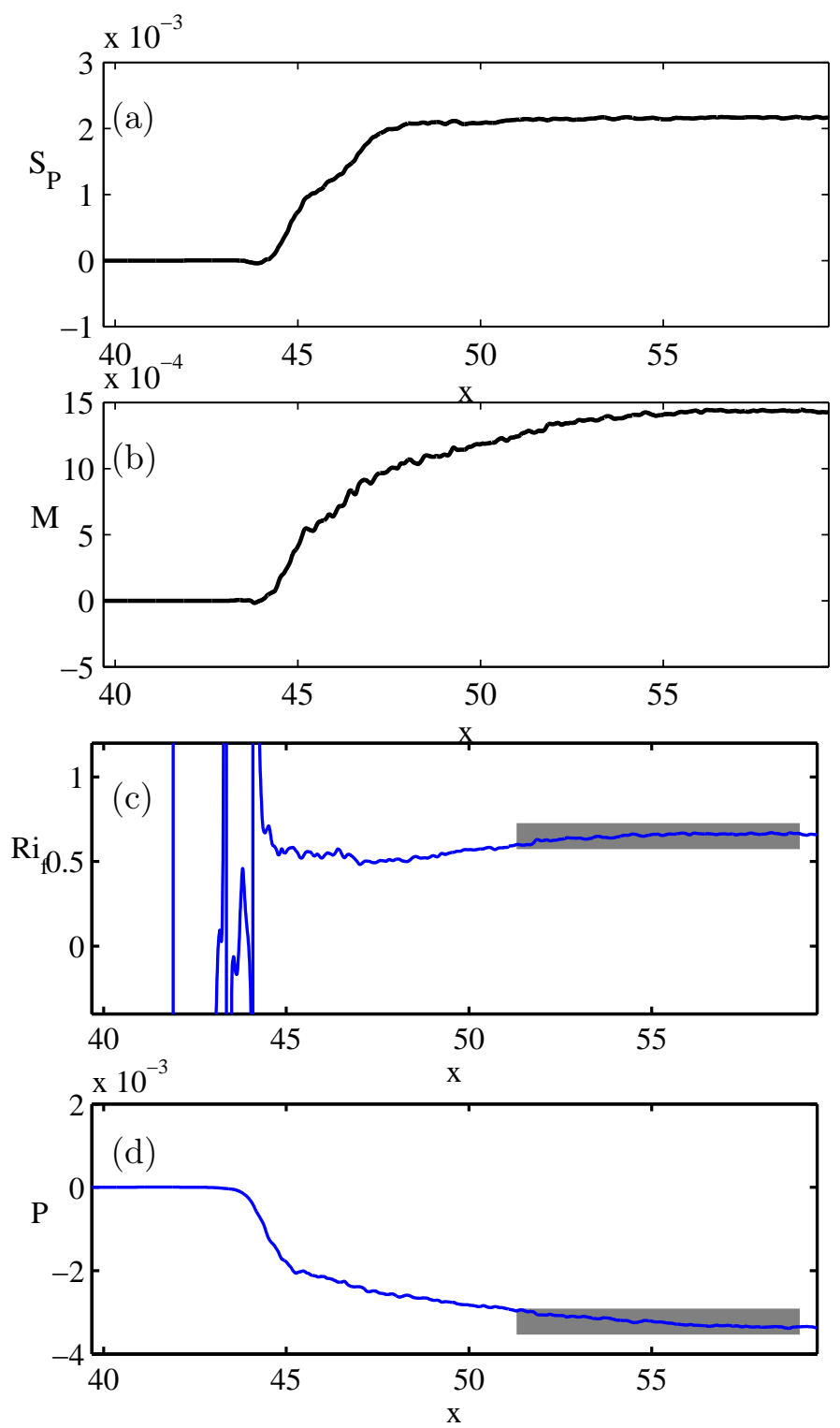

(e)
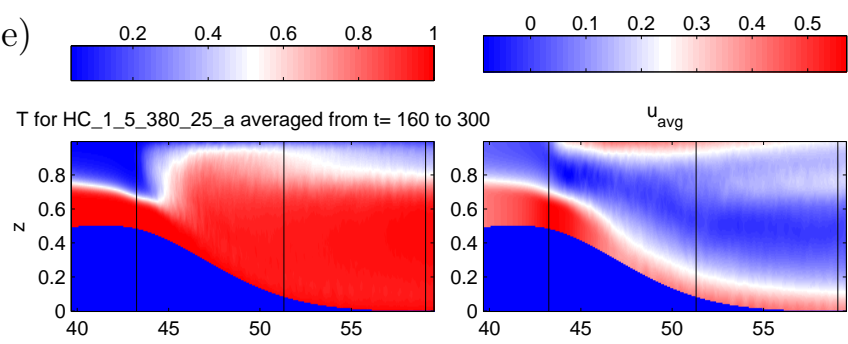

Figure 4-19: Cumulative integrals of (a) shear production of turbulent kinetic energy, (b) turbulent buoyancy flux, (c) Mixing efficiency, and (d) scalar variance production for a higher-mode jump with wedge $\left(q=0.1, h_{T}=0.5, T=380, \nu=7, R i_{f}=0.65, P=\right.$ $-0.0032)$. (d) Time-averaged scaled density field $T$ (left) and horizontal velocity field ( $u$, right) averaged from $t=160$ to $t=300$, with data every $\Delta t=2$. 
for a set of simulations. The plots of $P$ versus $T_{T}, \nu, h_{T}$, and the mode show that $P$ is not directly a function of any of these parameters. Note that the mode is determined by the highest mode of internal wave for which $c_{-}$changes from positive to negative across the jump, where internal wave speeds are calculated using the continuous density and velocity profiles using the Taylor-Goldstein equation (Appendix B).

The cumulative integral of scalar variance production is plotted versus upstream shear for a set of simulations in figure 4-21a. In this plot, color indicates the volume flow rate, which is correlated with shear because the flow over topography generates the shear. The figure uses the total depth as the length scale because that would be known when studying a naturally occuring internal hydraulic jumps (characteristic scales are given in 4.3.2). In chapters 2 and 3, the upstream lower-layer depth was used as the length scale, which would give non-dimensional shear values ranging from $0.2 \lesssim s \lesssim 1.3$ for the simulations shown in figure 4-21a. These shear values are consistent with the low shear jumps described in chapter 2. Figure 4-21b shows the scalar variance production versus upstream shear, with the mode of the jump indicated. Lower shear jumps tend to be higher-mode jumps with lower scalar variance production. While the shear and volume flow rate are highly correlated, the topographic height $h_{T}$ also affects the upstream shear that is generated, so flows with a lower volume flow rate (lower forcing) and lower topography generate less shear over the topography, producing jumps that are super-critical only to higher mode waves and have less mixing.

Jumps both with and without shear instabilities are included in figure 4-21. Although the mass balance ratios $\left(\frac{M_{a d v, 2}}{\Delta M_{2}}\right)$ of jumps with upstream shear are lower than those of jumps without upstream shear instabilities (overturning jumps or wedges), particularly at small $q$, the scalar variance production of all jumps follows the same trend. This suggests that upstream shear instabilities are not a controlling factor in the mixing produced by the jump; in first-mode jumps with upstream shear instabilities and jumps with wave overturning, shear instabilities develop within the jump, and the scalar variance production is similar for both types. At low volume flow 

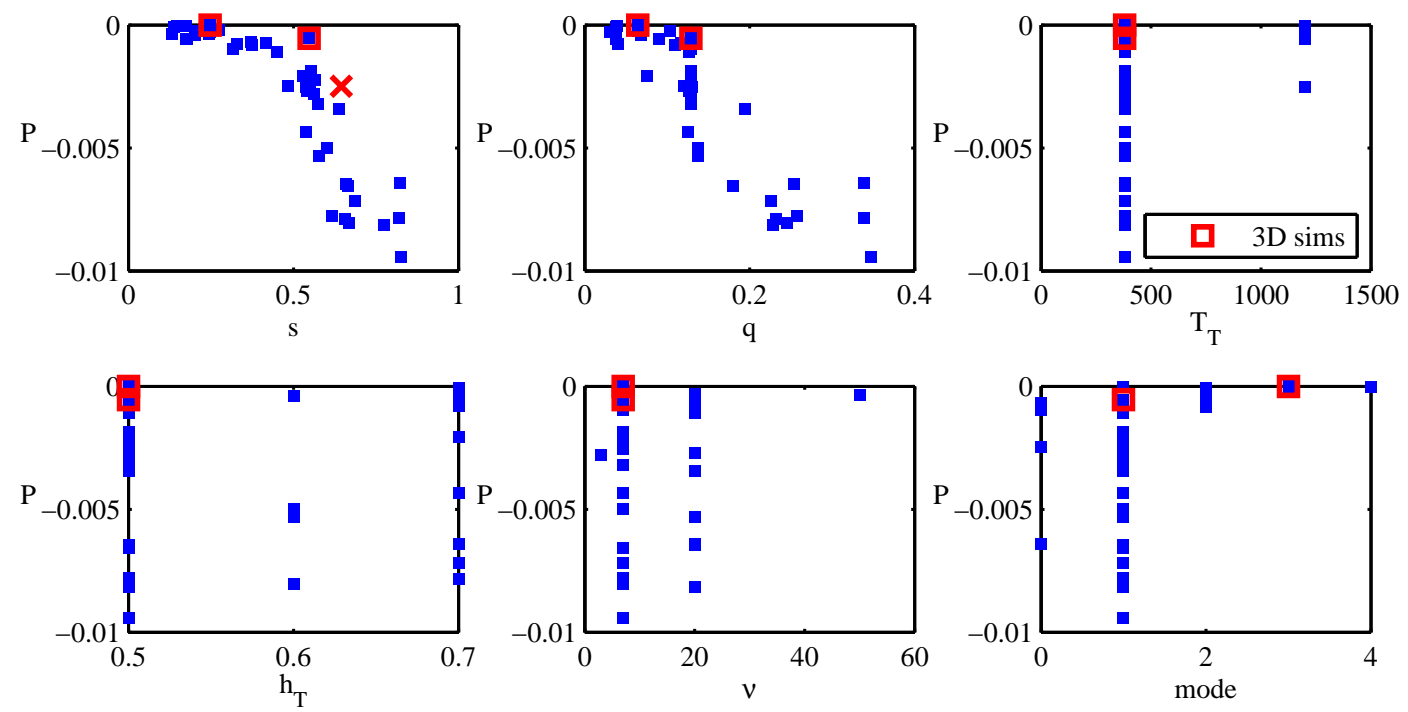

Figure 4-20: Scalar variance production $P$ as a function of the flow parameters $s, q, h_{T}, T_{T}$, $\nu$, and the mode of the jump. Stellwagen bank simulation result (see $\S 4.4$ ) is indicated by $\times$. 3D simulation results (see $\S 4.3 .7$ ) are indicated by $\square$.

rates, the upstream shear is small, so the scalar variance production is small for both wedge-type jumps and jumps that develop upstream shear instability.

While the relative contributions of advection and mixing to intermediate density water depend on whether upstream shear instability exists for jumps with a low volume flow rate, the scalar variance production follows the same trend for jumps both with and without upstream shear instability. This is illustrated in figure 4-22, which shows the scalar variance production versus upstream shear, with the jump type indicated. While it is interested to note which mechanism is responsible for mixing in a jump, the amount of mixing, when quantified by the scalar (density) variance production, appears to be independent or the jump type, varying mainly with upstream shear that develops.

When analyzing an internal hydraulic jump in the environment, parameters such as the upstream shear are unknown because it depends on where on the topography the jump forms. Known parameters include the volume flow rate and topographic features. Therefore, a regime diagram showing the scalar variance production in $q-h_{T}$ 

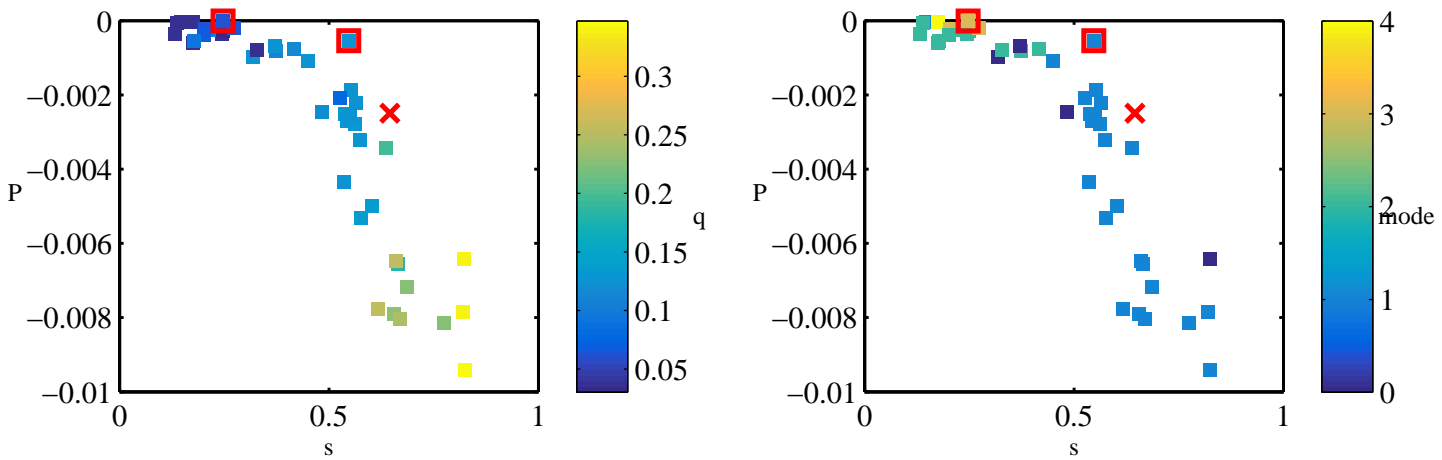

Figure 4-21: Cumulative integral of scalar variance production versus upstream shear. Color indicates volume flow rate (left), which is correlated with shear because the flow over topography generates the shear. Color indicates mode of jump (right), showing that higher mode jumps have lower scalar variance production, and therefore lower mixing. Stellwagen bank simulation result (see $\S 4.4$ ) is indicated by $\times$. 3D simulation results (see §4.3.7) are indicated by $\square$.

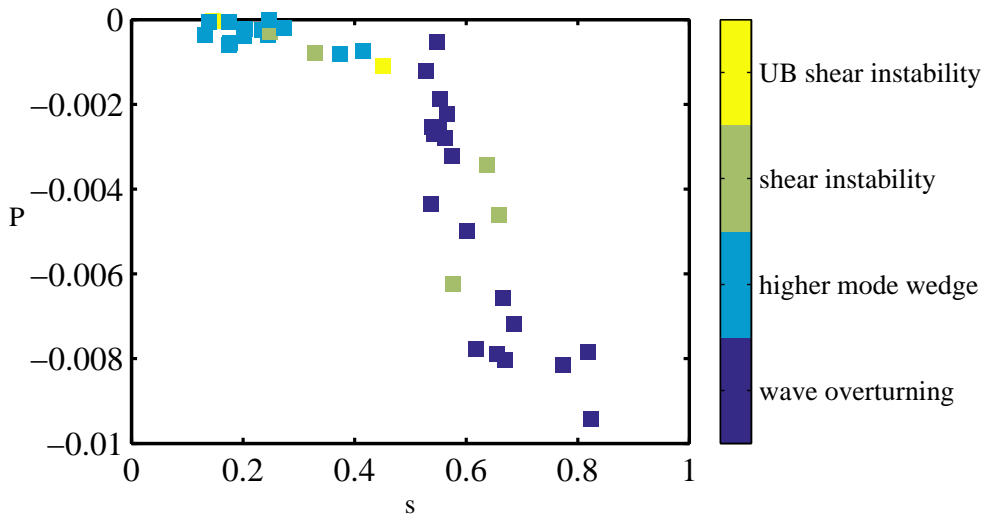

Figure 4-22: Scalar variance production versus upstream shear with jump type indicated. 

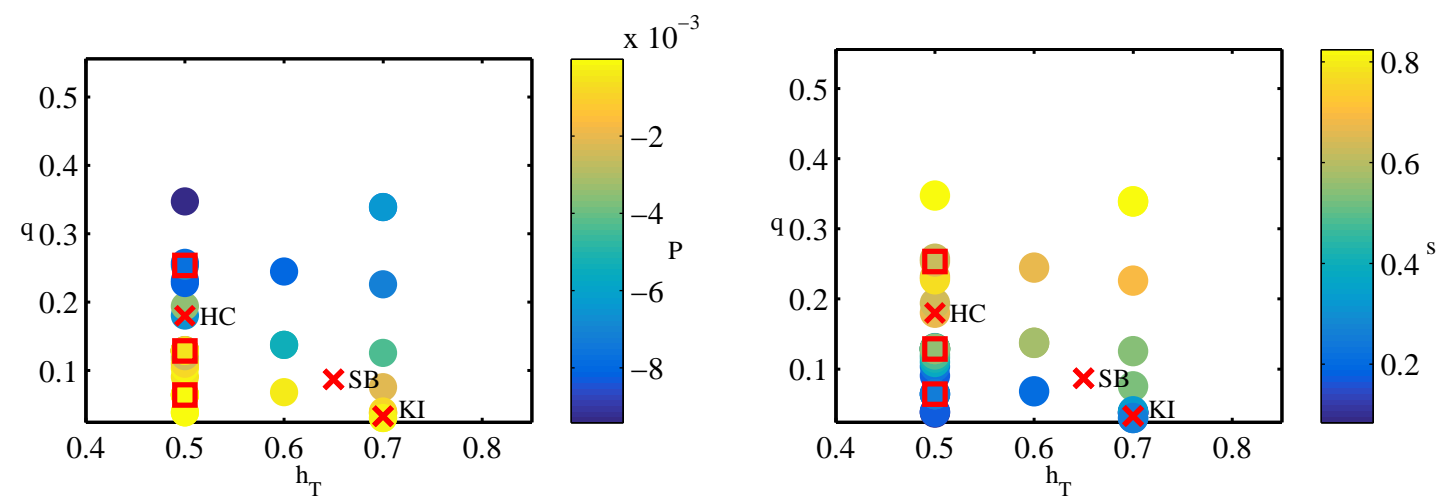

Figure 4-23: Regime diagram of $q$ versus $h_{T}$, with color indicating the scalar variance production (left) and the upstream shear (right). Increasing $h_{T}$ and/or $q$ increases both shear and scalar variance production (or mixing). $q$ and $h_{T}$ observations of Knight Inlet $(\mathrm{KI})$, Hood Canal (HC), and Stellwagen Bank (SB) are indicated by $\times$. 3D simulation results (see $\S 4.3 .7$ ) are indicated by $\square$ surrounding the colored symbol.

space is shown in figure 4-23. Although upstream shear $s$ depends on topographic height $h_{T}$ (figure 4-23), the dependence on $q$ is much stronger.

\subsubsection{Simulations with viscosity}

The simulations shown so far in this chapter have not included explicit viscosity and no-slip bottom boundaries, which together will be referred to as no-slip and can have important effects on flows with topography. In chapter 2, simulations with viscosity $(R e=500,1250$, and 4000) and a free-slip bottom boundary condition were performed and were found to be very similar to flows without explicit viscosity. However, when the flow is over topography, the no-slip condition may cause flow separation, altering the path of the descending dense flow (Lamb, 2004). Cummins (2000) simulates bottom boundary layer separation using modified topography that forces the bottom jet to follow the appropriate path that a separated jet would follow in a no-slip flow. While the precise shape of topography that would force the flow to follow the appropriate separated path is unknown a priori, the previous simulations described in this work could be considered as having modified topography and are therefore still relevant for categorizing the qualitative types of jumps that occur and studying mixing trends and mass budgets. However, features such as shear instabilities along the underside of the separating jet could impact the flow in a way that 


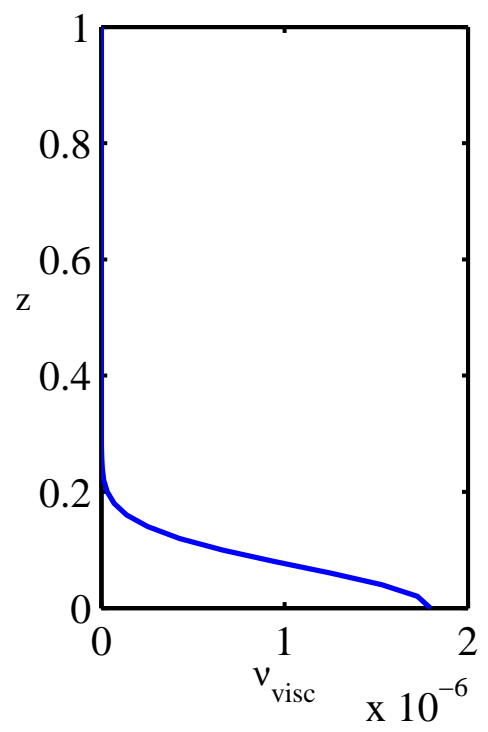

Figure 4-24: Viscosity function.

cannot be accounted for by modified topography, so a few no-slip simulations are also conducted and described here.

The no-slip condition in these simulations includes a spatially varying viscosity profile given by

$$
\nu_{v i s c}=1.8 \times 10^{-6} \times e^{-\frac{\left(y-h_{b}\right)^{2}}{0.1^{2}}}
$$

and shown in figure 4-24, along with a no-slip bottom boundary condition over the topography. The viscosity is larger near the bottom topography and decreases to zero away from the topography so that it can cause flow separation without significantly affecting the interior of the flow. The magnitude of the viscosity and the decay rate were chosen somewhat arbitrarily; the simulations (discussed below) show that flow separation occurs without significantly affecting the structure of the jump, so the values chosen are acceptable. Explicit diffusivity is not included.

An example no-slip simulation is shown in figure 4-25 $\left(q=0.13, T_{T}=380, h_{T}=\right.$ $\left.0.5, \nu=7, \lambda=10, P=-0.0026, R i_{f}=0.55, \frac{M_{a d v, 2}}{\Delta M_{2}}=0.41\right)$. The time-averaged tracer and velocity fields are shown. The first three modes of the internal wave speeds are also shown along the domain, calculated from the continuous profiles using the 
Taylor-Goldstein equation. Vertical profiles of $T$ and $u$ are shown at three locations along the domain; one location is just upstream of the jump and the other two are downstream. The equivalent inviscid simulation is shown in the lower half of figure $4-25\left(q=0.13, T_{T}=380, h_{T}=0.5, \quad \nu=7, \lambda=10, P=-0.0032, R i_{f}=\right.$ $\left.0.65 \frac{M_{a d v, 2}}{\Delta M_{2}}=0.48\right)$. The path of the flow is significantly different in the case the noslip condition; the flow separates from the topography and the jet enters the interior of the domain before breaking up, instead of forming a persistent jet that follows the topography, as in the inviscid case. This is apparent in the velocity field as well as the velocity profiles, which show that the velocity is zero near the topography in the no-slip flows. The separated jet is also visible in the downstream velocity profiles, whereas the velocity profiles for flows without viscosity show large velocities at the bottom through the bottom jet. The scaled density profiles of the no-slip flows, however, are similar to the profiles for the flows without viscosity.

In no-slip flows, shear instabilities form on the underside of the jet, seen in figure 4-26 which shows the instantaneous streamlines and $R i_{g}$ at two times during the development of the jump for both the no-slip simulation and the simulation without viscosity. The no-slip condition also appear to slightly delay wave overturning, which is is identified as the time at which $M_{m i x, 2}$ begins to increase quickly. This is seen in figure 4-27, which shows the mass budget for the no-slip simulation and the simulation without viscosity. Mixing due to wave overturning begins at $t \approx 140$ in the simulation without viscosity, and at $t \approx 150$ in the no-slip simulation, although this is very small difference. However, the no-slip condition and the resulting bottom boundary layer separation do not appear to change the jump type to a jump with upstream shear instability, although the leading edge of the jump is qualitatively different (figure 4-26) due to the oscillations in the separated jet starting at $x \approx 45$ just above the topography. The mass budgets for these two simulations (figure 4-27) show that the advection and mixing between water masses is similar in both the magnitude and the source (advection or mixing).

A higher-mode jump with the no-slip condition $\left(q=0.064, T_{T}=380, h_{T}=\right.$ 
(a)

(b)

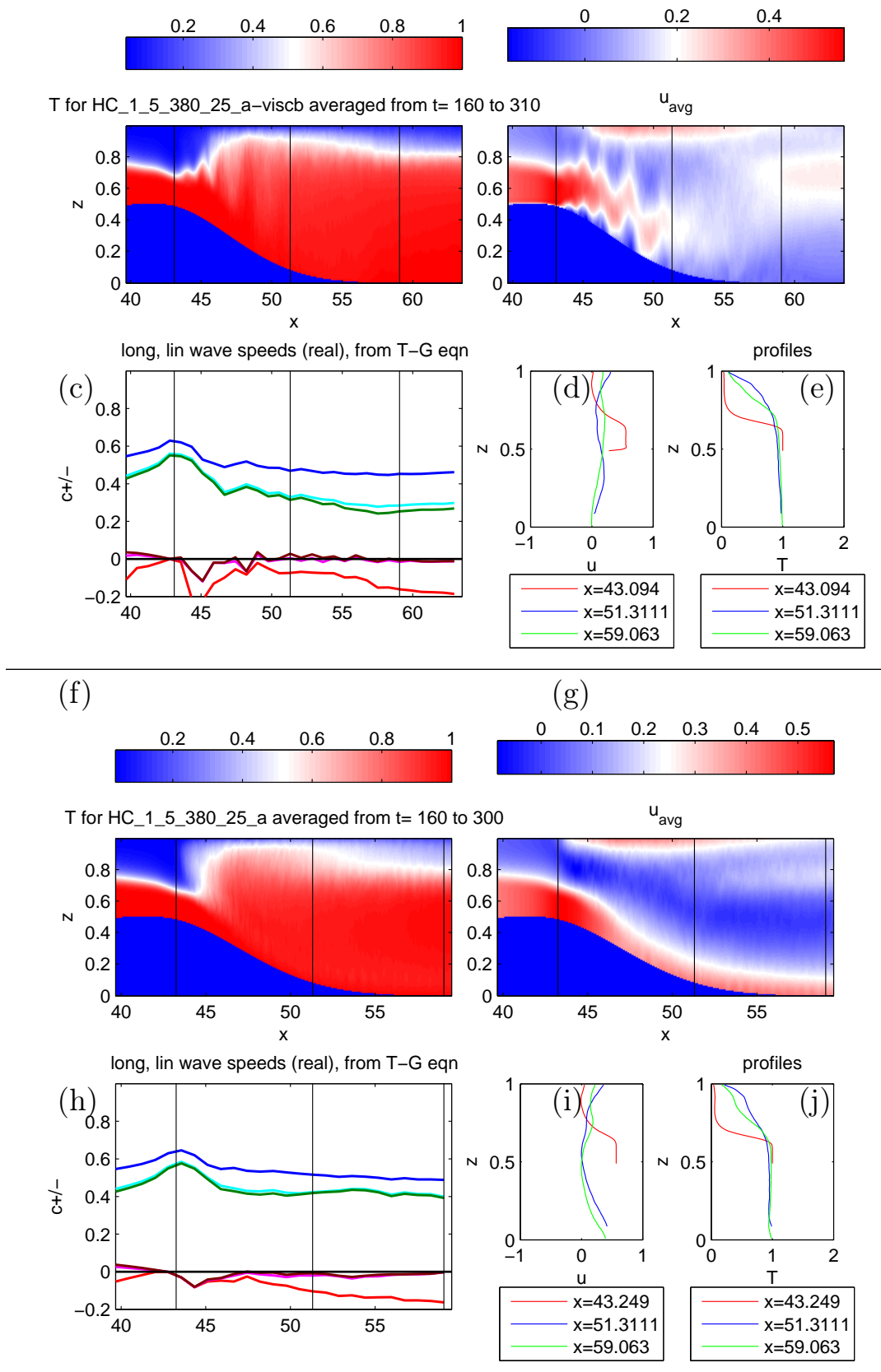

Figure 4-25: Time-averaged results for a no-slip flow ((a)-(e), $q=0.13, T_{T}=380, h_{T}=$ $\left.0.5, \quad \nu=7, \lambda=10, P=-0.0026, R i_{f}=0.55, \frac{M_{a d v, 2}}{\Delta M_{2}}=0.41\right)$ and a flow without viscosity $\left((\mathrm{f})-(\mathrm{j}), q=0.13, T_{T}=380, h_{T}=0.5, \nu=7, \lambda=10, P=-0.0032, R i_{f}=\right.$ $0.65 \frac{M_{a d v, 2}}{\Delta M_{2}}=0.48$ ). (a) and (f) show the time averaged, scaled density field, (b) and (g) show the horizontal velocity field, (c) and (h) show $c_{ \pm}$of the first two modes of the long internal wave speeds, (d) and (i) show vertical velocity profiles at three locations along the domain, indicated by vertical lines in the density and velocity plots, and (e) and (j) shown vertical density profiles at the same three locations. 

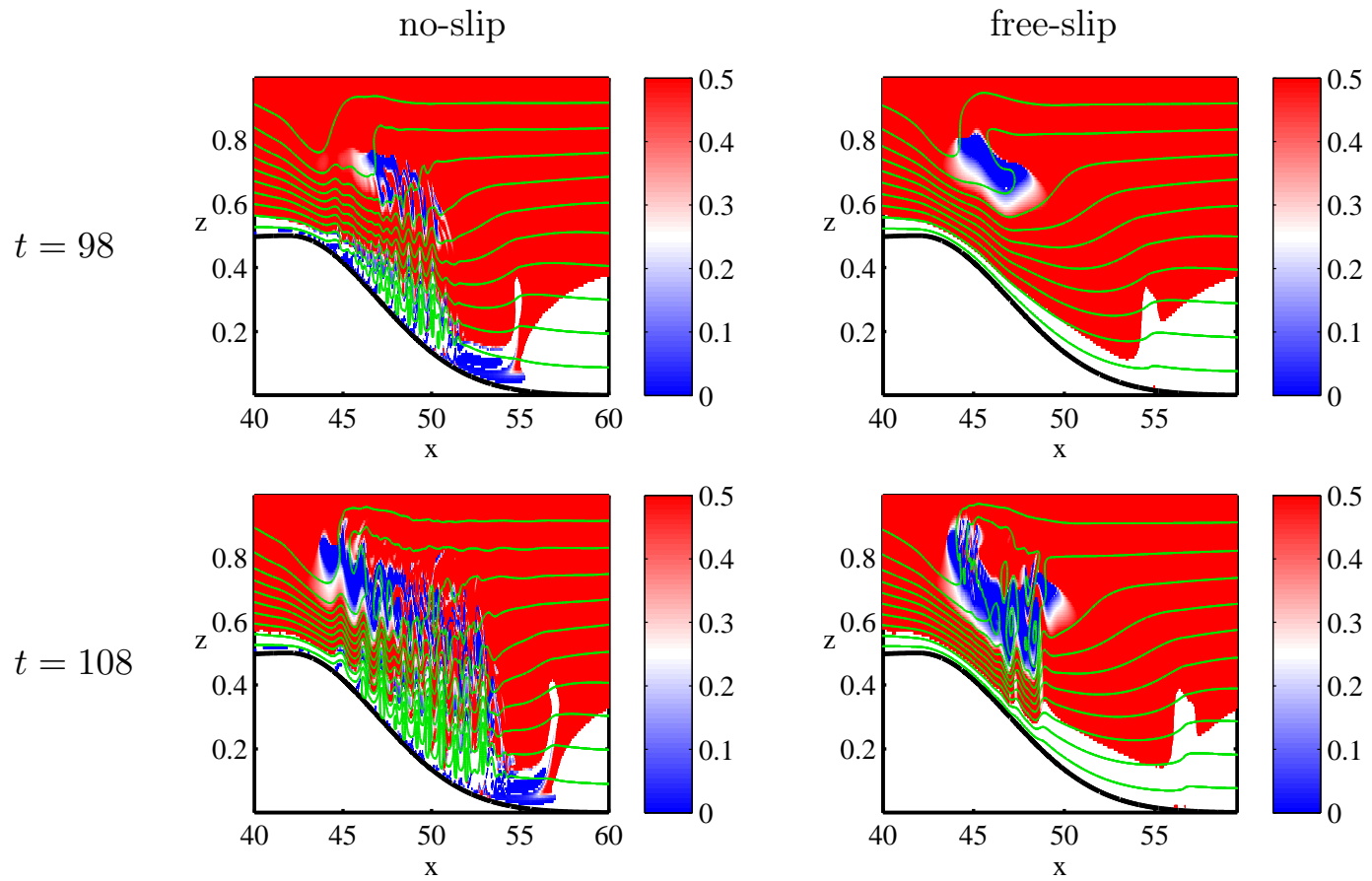

Figure 4-26: Richardson number and streamlines for flows with (left, $q=0.13, T_{T}=$ $\left.380, h_{T}=0.5, \nu=7, \lambda=10, P=-0.0026, R i_{f}=0.55, \frac{M_{a d v, 2}}{\Delta M_{2}}=0.41\right)$ and without (right, $q=0.13, T_{T}=380, h_{T}=0.5, \nu=7, \lambda=10, P=-0.0032, R i_{f}=0.65 \frac{M_{a d v, 2}}{\Delta M_{2}}=$ 0.48 ) no-slip condition at $t=108$ (top) and $t=124$ (bottom).

$\left.0.5, \quad \nu=7, \lambda=10, P=-0.00019, R i_{f}=0.22, \frac{M_{a d v, 2}}{\Delta M_{2}}=1.0\right)$ is compared to a simulation without explicit viscosity $\left(q=0.065, T_{T}=380, h_{T}=0.5, \nu=7, \lambda=\right.$ 10, $\left.P=-0.00023, \frac{M_{a d v, 2}}{\Delta M_{2}}=1.1\right)$ in figure 4-28. In this case, the jump occurs upstream of the location where the jet separates from the topography, so the jump itself is very similar in both cases. Again, flow separation occurs with the no-slip condition, so the velocity field is qualitatively different, although mainly downstream of the jump. The velocity field and profiles show a bottom jet with the largest velocity along the bottom topography in the flow without viscosity, whereas the no-slip flow develops a separated jet at $x \approx 44$, which flows into the interior of the domain. Furthermore, in the no-slip flow, the velocity is zero along the topography, as seen in the velocity profiles.

Jumps with upstream shear instabilities with and without the no-slip condition are shown in figure 4-29. The free-slip jump in figure 4-29 is also shown in figure 4-9, which shows the upstream shear instabilities more clearly in the instantaneous fields. 

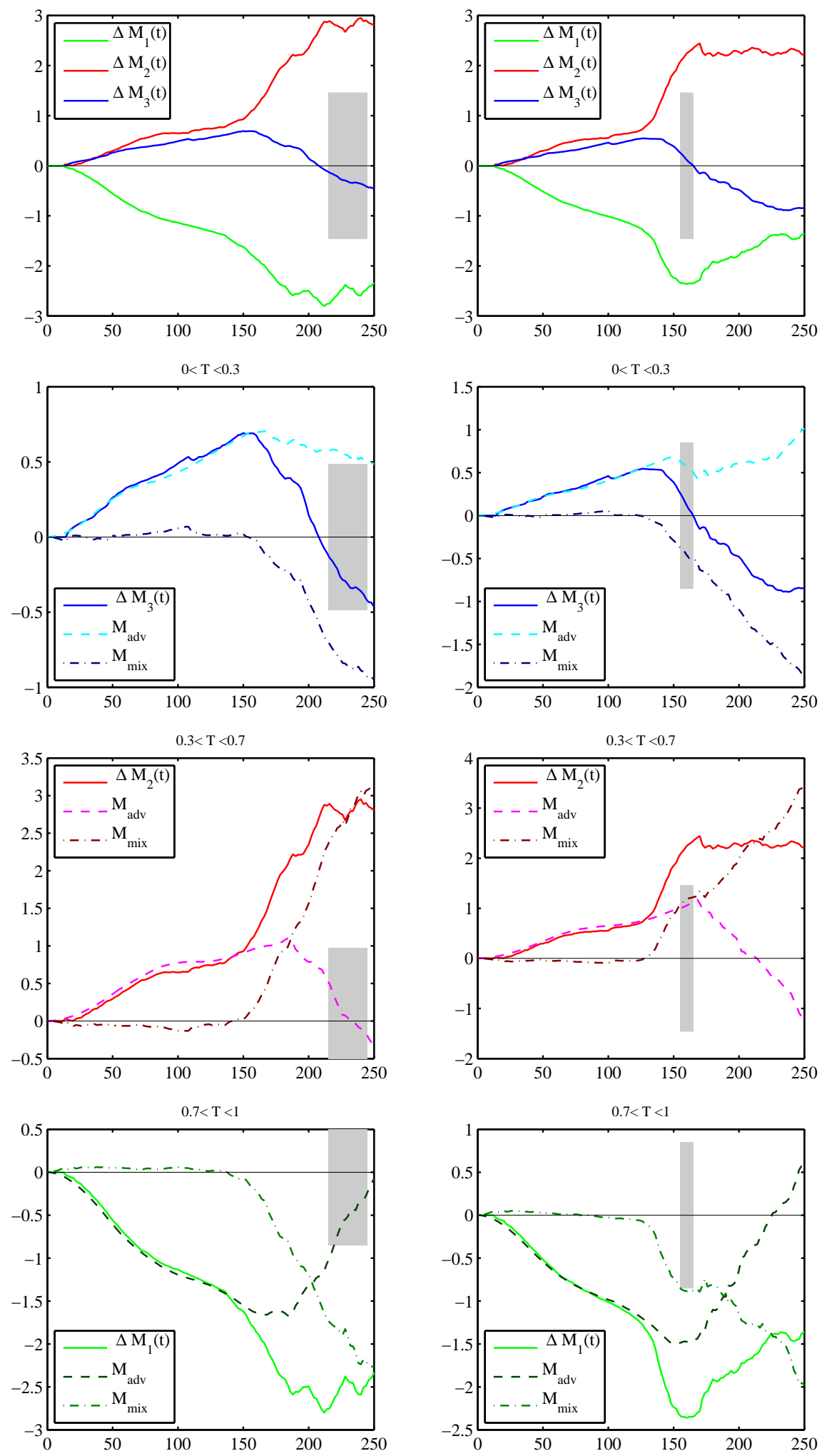

Figure 4-27: Water mass development with time (top) for each water mass. Second (third, fourth) row shows light (middle, heavy) water mass budget with contributions from advection and mixing. No-slip (left, $q=0.13, T_{T}=380, h_{T}=0.5, \nu=7, \lambda=10, P=$ $-0.0026, R i_{f}=0.55, \frac{M_{a d v, 2}}{\Delta M_{2}}=0.41$ ) and inviscid (right, $q=0.13, T_{T}=380, h_{T}=$ $\left.0.5, \nu=7, \lambda=10, P=-0.0032, R i_{f}=0.652 \frac{M_{a d v, 2}}{\Delta M_{2}}=0.48\right) . T=380, h_{T}=0.5$. 
(a)

(b)

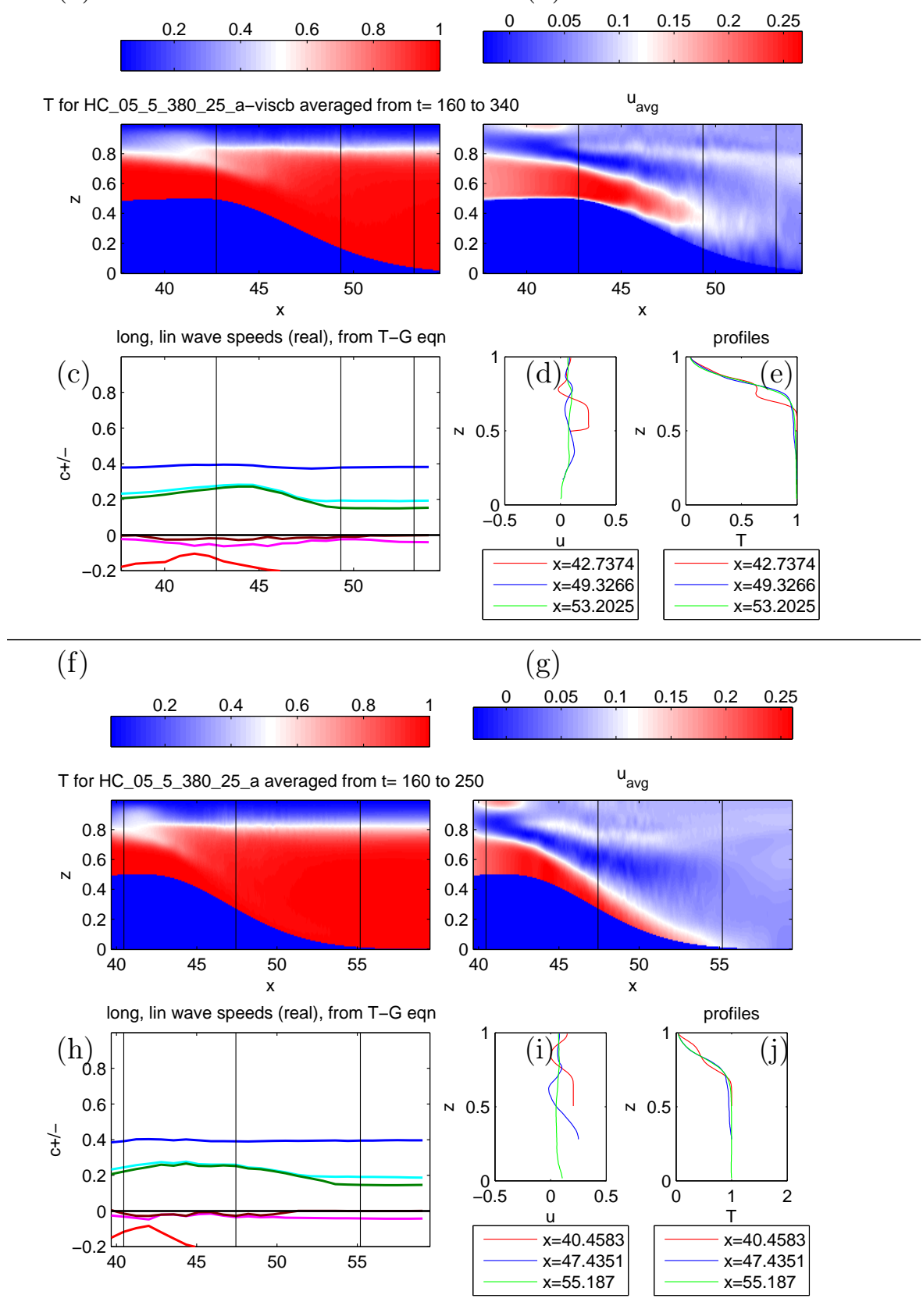

Figure 4-28: Time-averaged results for a no-slip flow ((a)-(e), $q=0.064, T_{T}=380, h_{T}=$ $\left.0.5, \nu=7, \lambda=10, P=-0.00019, R i_{f}=0.22, \frac{M_{a d v, 2}}{\Delta M_{2}}=1.0\right)$ and a flow without viscosity $\left((\mathrm{f})-(\mathrm{j}), q=0.065, T_{T}=380, h_{T}=0.5, \nu=7, \lambda=10, P=-0.00023, \frac{M_{a d v, 2}}{\Delta M_{2}}=1.1\right)$. (a) and (f) show the time averaged, scaled density field, (b) and (g) show the horizontal velocity field, (c) and (h) show $c_{ \pm}$of the first two modes of the long internal wave speeds, (d) and (i) show vertical velocity profiles at three locations along the domain, indicated by vertical lines in the density and velocity plots, and (e) and (j) shown vertical density profiles at the same three locations. 
Again, the bottom jet separates in the no-slip case, although the jump type does not change. The jump front is influenced by the instabilities on the underside of the separating jet, resulting a more gradual jump. However, the jump structure is otherwise similar.

Farmer and Armi (2001) conclude that bottom boundary layer separation can change the jump type from an overturning wave to a jump with upstream shear instability. This possibility is investigated by conducting two simulations that are equivalent except for the use of the no-slip condition in one and a free-slip bottom in the other. The results are presented in figure 4-30, which shows a first-mode jump with a thin interface but steep bottom topography with the no-slip condition. The equivalent case without viscosity is also shown in figure 4-30. Again, the velocity field is qualitatively different due to bottom boundary layer separation. However, in this case, the bottom boundary layer separation provides an effectively gentler topographic slope, allowing shear instabilities to develop just upstream of the jump. The upstream shear instabilities are difficult to identify in figure 4-30, although they are visible at $x \approx 46$ and $z \approx 0.6$ in figure 4-31, which shows instantaneous $R i_{g}$ and streamlines at two times for both the no-slip and free-slip cases. The equivalent flow without viscosity does not exhibit upstream shear instabilities. This change in jump types between the no-slip and free-slip flows seen in figures 4-30 and 4-31 is possible because the upstream interface is thin and unstable to shear instabilities, and steep topography is the only parameter preventing them from developing in the free-slip flow.

The two no-slip simulations shown in figures $4-25$ and $4-28$ are included in figures 4-19 and 4-23, which show that mixing increases with increasing upstream shear, and figure 4-15, which shows that the contribution of advection divergence to the intermediate mass budget decreases as volume flow rate increases. The no-slip simulations follow the same trends as the simulations without explicit viscosity. 
(a)

(b)

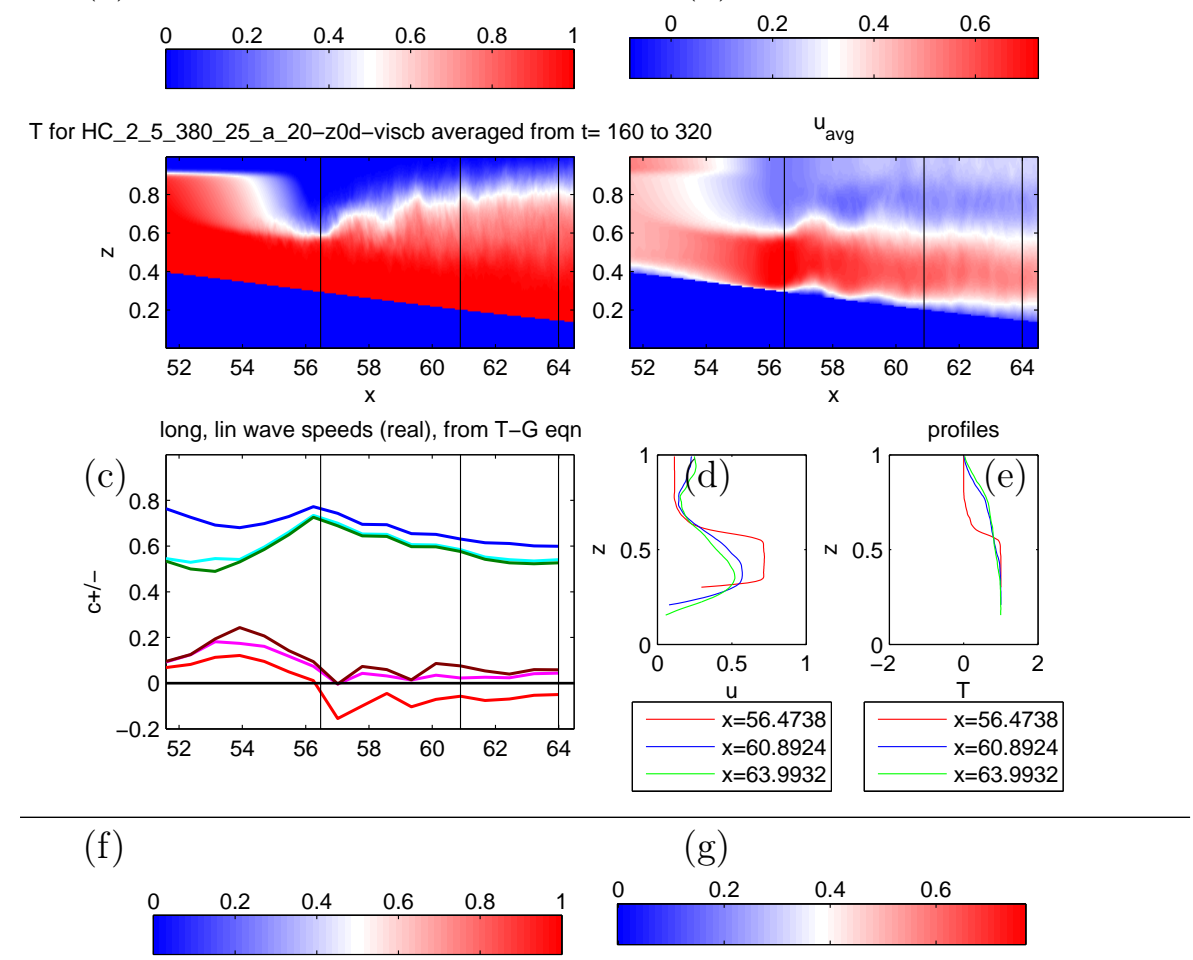

T for HC_2_5_380_25_a_20-z0d-coarse averaged from t= 160 to $340 \quad u_{\text {avg }}$
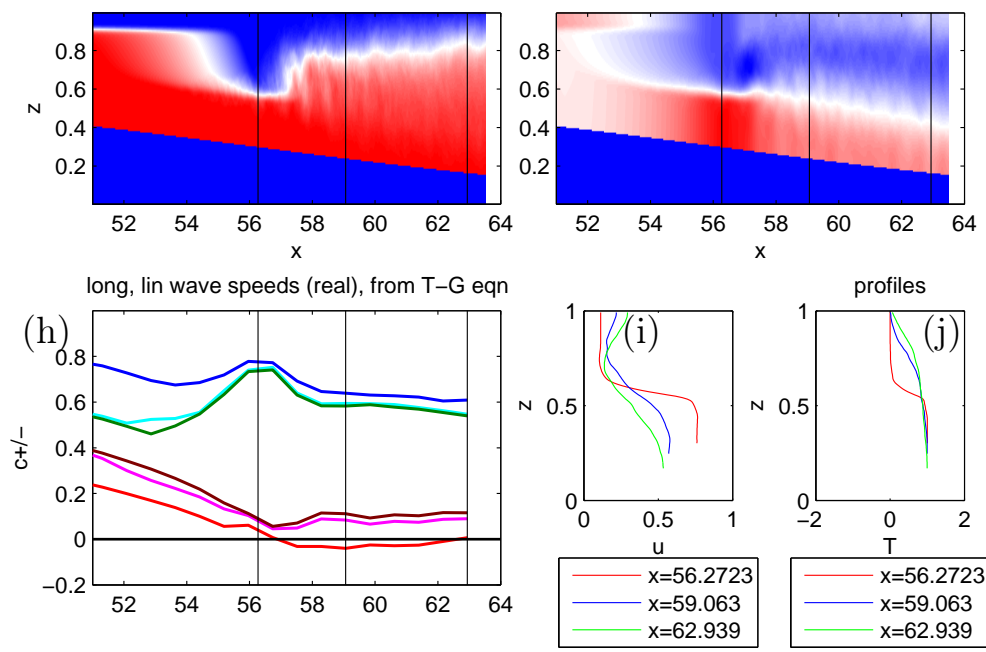

Figure 4-29: Time-averaged results for a first-mode jump with no-slip condition ((a)-(e), $\left.q=0.25, T_{T}=380, h_{T}=0.5, \nu=20, \lambda=90\right)$ and an equivalent flow without viscosity ((f)-(j), $\left.q=0.25, T_{T}=380, h_{T}=0.5, \nu=20, \lambda=90\right) . \quad$ (a) and (f) show the time averaged, scaled density field, (b) and (g) show the horizontal velocity field, (c) and (h) show $c_{ \pm}$of the first two modes of the long internal wave speeds, (d) and (i) show vertical velocity profiles at three locations along the domain, indicated by vertical lines in the density and velocity plots, and (e) and (j) shown vertical density profiles at the same three locations. 
(a)

(b)
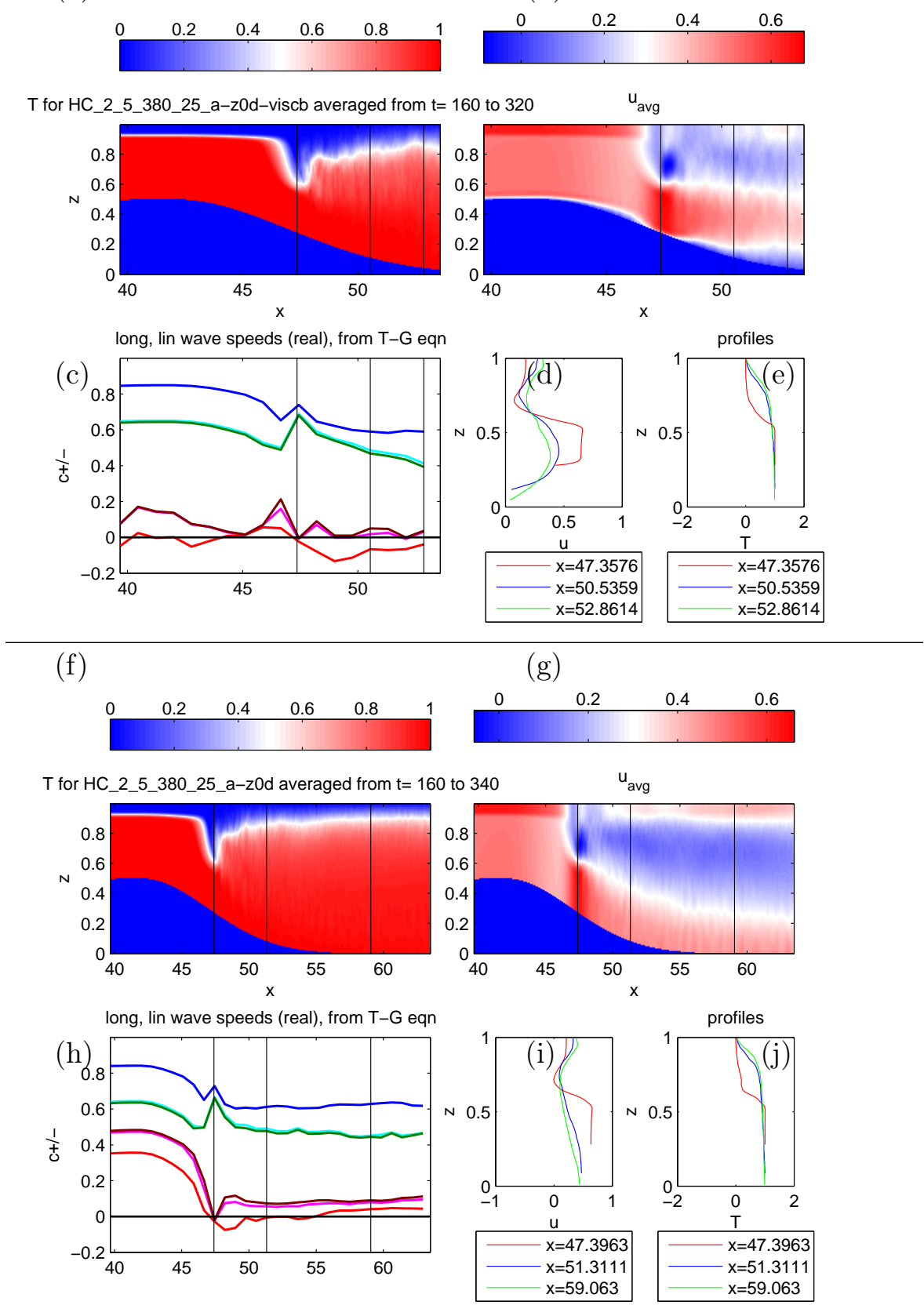

Figure 4-30: Time-averaged results for a first-mode jumps with the no-slip condition. ((a)(e), $\left.q=0.25, T_{T}=380, h_{T}=0.5, \nu=7, \lambda=90\right)$ and an equivalent flow without viscosity ((f)-(j)) that produces an overturning wave. (a) and (f) show the time averaged, scaled density field, (b) and (g) show the horizontal velocity field, (c) and (h) show $c_{ \pm}$of the first two modes of the long internal wave speeds, (d) and (i) show vertical velocity profiles at three locations along the domain, indicated by vertical lines in the density and velocity plots, and (e) and (j) shown vertical density profiles at the same three locations. 


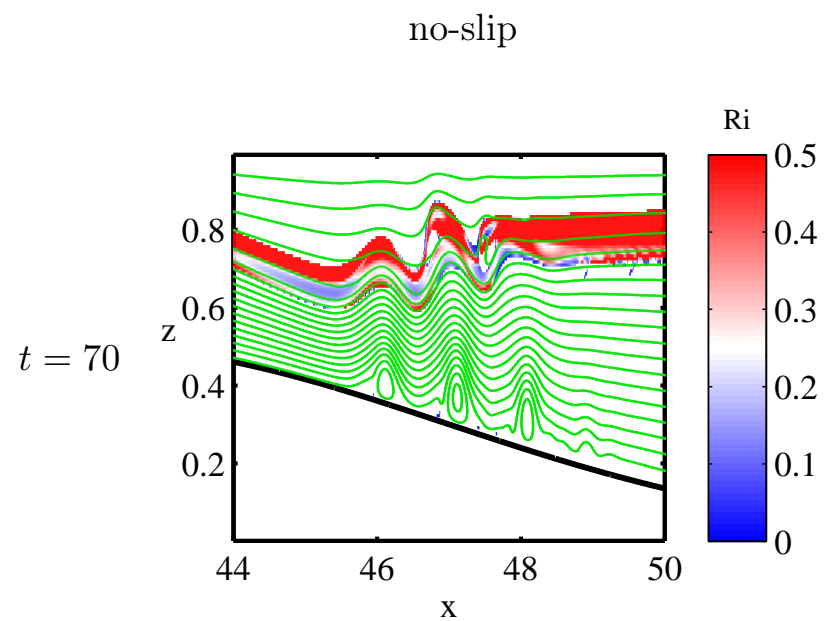

free-slip
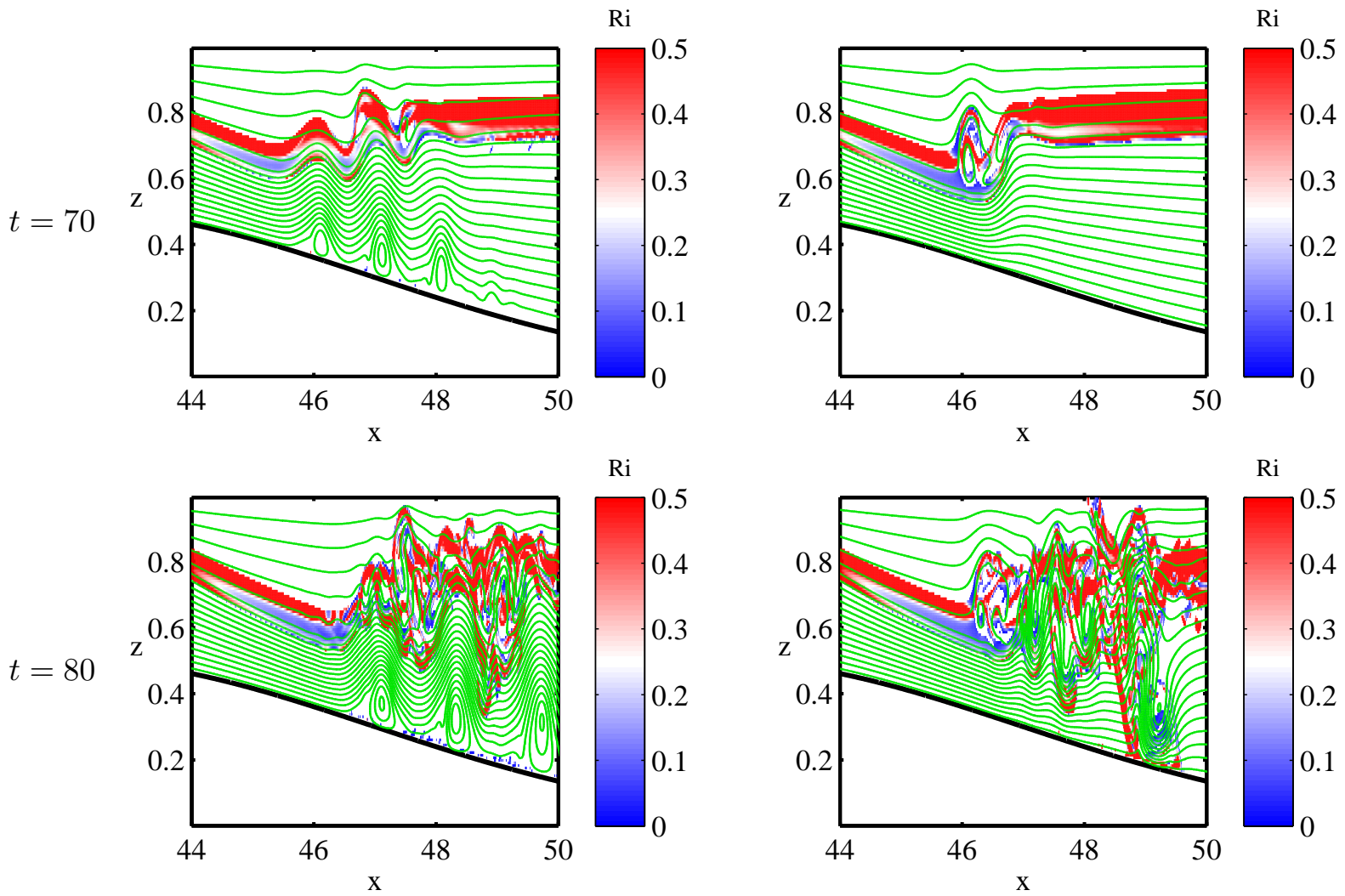

$\mathrm{Ri}$

Ri

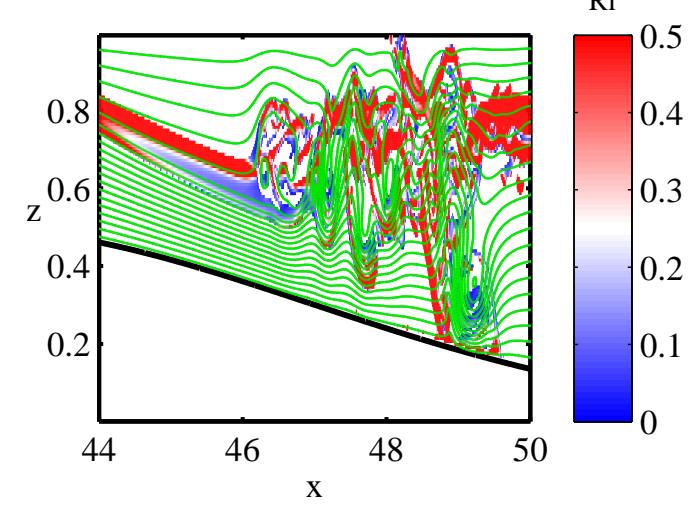

Figure 4-31: $R i_{g}$ and streamlines for flow with (left) and without (right) the no-slip condition at $t=70$ (top) and $t=80$ (bottom). Results correspond to the simulations shown in figure 4-30. 


\subsubsection{D Simulations}

The stratification, forcing, and geometry found in locations such as Knight Inlet and Hood Canal, and used in these simulations, results in flows with small upstream shear consistent with the jumps described in chapter 2 . Therefore, it is expected that threedimensional jumps in flows over topography will be consistent with the $2 \mathrm{D}$ results found here, just as the $2 \mathrm{D}$ and $3 \mathrm{D}$ jumps were consistent in chapter 2. Several 3D simulations are compared to the equivalent $2 \mathrm{D}$ simulations to determine whether this is the case.

The 3D simulations have a channel width equal to the height of the domain, which allows 3D turbulence to develop. However, the cross-channel width prevents larger lateral features from developing in the flow, and the topography does not vary in the cross-channel direction. The resolution is 128 points in the vertical in the region of the jump, and 64 points in the vertical throughout the rest of the domain with $\Delta x=\Delta y=\Delta z$. There is a periodic boundary condition with no topographic variation in the transverse direction. The simulations are otherwise equivalent to the corresponding 2D simulations. Simulations without explicit viscosity or diffusivity are considered here.

A 3D mode-1 internal hydraulic jump with wave overturning is shown in figure 4$32\left(q=0.13, h_{T}=0.5, T_{T}=380, \nu=7, \lambda=10, P=-0.0005\right.$, and $\left.\frac{M_{a d v, 2}}{\Delta M_{2}}=0.7373\right)$. The magnitude of the velocity and the velocity vector field are shown in the top panel of figure 4-32, density contours and isopycnals can be seen in the second panel from the top, and the instantaneous water masses, defined in $\S 4.3 .4$, are shown in the third panel from the top. The change in each water mass with time is shown for all three water masses in the bottom panel, and the vertical line indicates the time at which the instantaneous plots were taken. Similar plots for the equivalent $2 \mathrm{D}$ simulation are seen in the right column of figure $4-32\left(P=-0.0032\right.$ and $\left.\frac{M_{a d v, 2}}{\Delta M_{2}}=0.48\right)$.

The jumps form in approximately the same location in both cases, with similar jump structures. As in $\S 2.5,2 \mathrm{D}$ vortices break down quickly in $3 \mathrm{D}$, resulting in 
$3 \mathrm{D}$
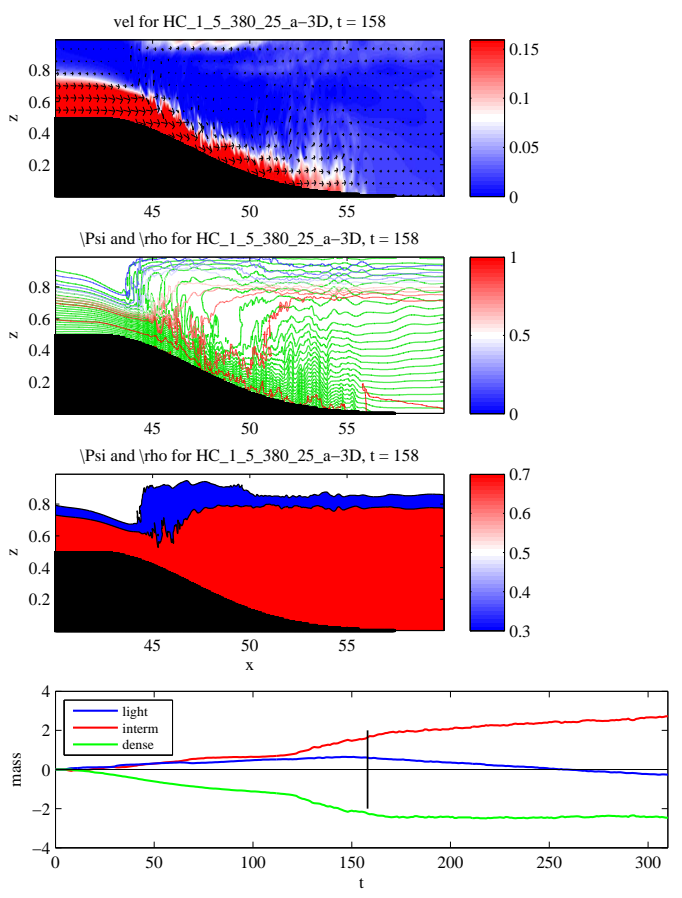

$2 \mathrm{D}$
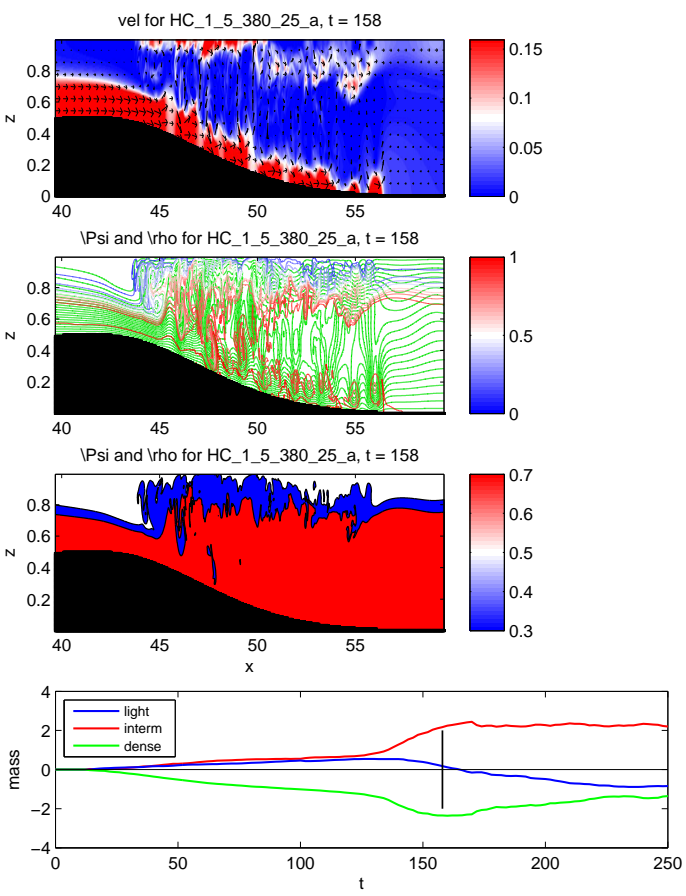

Figure 4-32: $3 \mathrm{D}$ (top, $P=-0.0005, \frac{M_{a d v, 2}}{\Delta M_{2}}=0.7373$ ) and 2D (bottom, $P=-0.0032$, $\left.\frac{M_{a d v, 2}}{\Delta M_{2}}=0.48\right)$ equivalent simulations of a first-mode jump with wave overturning. $q=0.13$, $h_{T}=0.5, T_{T}=380, \nu=7$, and $\lambda=10$. Top panel shows horizontal velocity field and vector field. Second panel from top shows density contours and streamlines. Third panel from top identifies the three water masses. Bottom panel shoes $\Delta M_{i}(t)$ for each of the three water masses. 
sharper interfaces. Mixing is also less efficient in 3D, resulting in a slightly larger advective term compared to the mixing term in the mass balance (figure 4-15), although the ratio is still similar to those found in $2 \mathrm{D}$ simulations. The scalar variance production is significantly smaller in 3D (figure 4-19) than in 2D, which is consistent with the smaller mixing efficiencies seen in $3 \mathrm{D}$ simulations in $\S 2.5$, compared to the equivalent $2 \mathrm{D}$ simulations. The flux Richardson number for this 3D simulation (figure $\left.4-26, R i_{f} \approx 0.07\right)$ is smaller than the values seen in the idealized calculation of $\S 2.5$, most likely due to upstream adjustment as discussed in $\S 4.3 .5$, which is why the scalar variance production is considered here. The mass balance ratio, $\frac{M_{a d v, 2}}{\Delta M_{2}}$, is also somewhat smaller, but still within the scatter of similar 2D simulations. Otherwise, the simulations are very similar, suggesting that results from the $2 \mathrm{D}$ simulations also apply to 3D flows and to hydraulic jumps found in the environment. The differences between 2D and 3D in these simulations with topography are similar to the differences between 2D and 3D found in the idealized simulations of $\S 2.5$.

A 3D simulation of a higher-mode jump with a wedge $\left(q=0.065, T_{T}=380, h_{T}=\right.$ $\left.0.5, \nu=7, \lambda=10, P=-0.000007, \frac{M_{a d v, 2}}{\Delta M_{2}}=1.2\right)$ is compared to the equivalent $2 \mathrm{D}$ simulation $\left(P=-0.00023, \frac{M_{a d v, 2}}{\Delta M_{2}}=1.1\right)$ in figure 4-33. The jumps are qualitatively very similar with the expected quantitative differences in $P$ and $\frac{M_{a d v, 2}}{\Delta M_{2}}$, as with the first-mode jump. This is again consistent with the results from $§ 2.5$, which is expected because these jumps over topography are low shear jumps, with shear values in the same range as those considered in chapter 2 . These results suggest that the findings from this chapter can be directly extended to 3D, with the scalar variance production and mixing efficieny appropriately reduced. 
$3 \mathrm{D}$

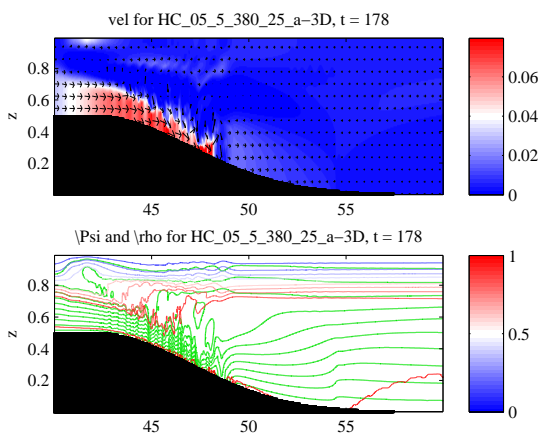

PPsi and $\backslash$ rho for HC_05_5_380_25_a-3D, $t=178$
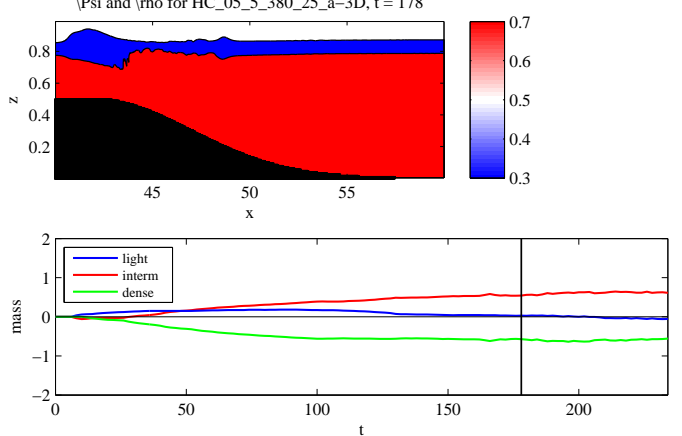

$2 \mathrm{D}$
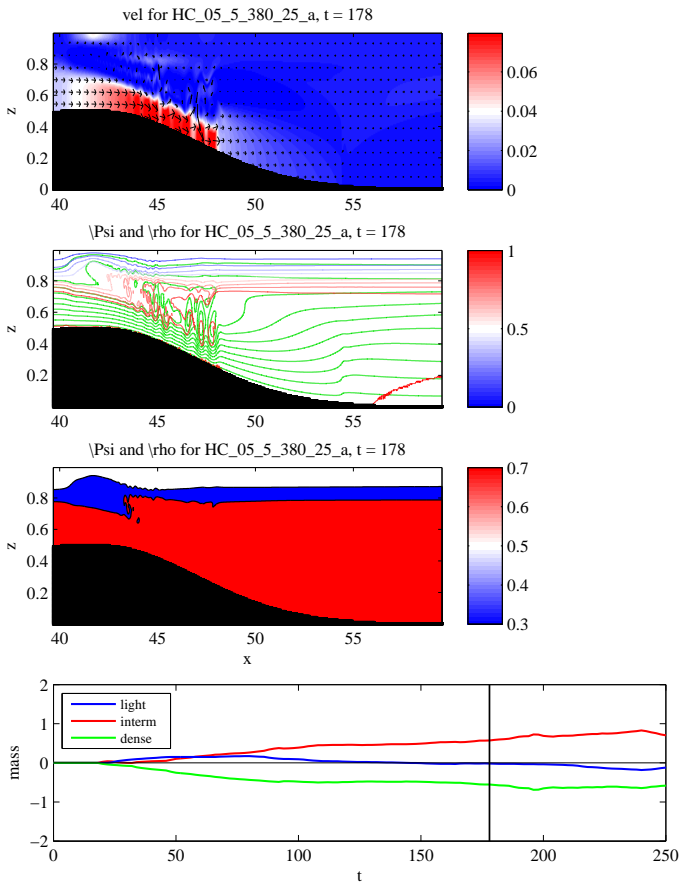

Figure 4-33: 3D (top) and 2D (bottom) equivalent simulations of a higher-mode jump with a wedge. $q=0.06, h_{T}=0.5, T_{T}=380, \nu=7$, and $\lambda=10$. Top panel shows horizontal velocity field and vector field. Second panel from top shows density contours and streamlines. Third panel from top identifies the three water masses. Bottom panel shoes $\Delta M_{i}(t)$ for each of the three water masses. 


\subsection{Stellwagen Bank}

Observations of the flow over a sill in Stellwagen Bank are shown in figure 4-34 (Pineda, personnal communication). The flow over Stellwagen is tidally driven, and the M2 (semi-diurnal lunar) tidal component is dominant. As noted in $\S 1.1$, flow over the sill results in a hydraulic jump forming in the lee of the sill. Figure 4-34a shows acoustic backscatter, and figure 4-34b shows the velocity field (negative velocities are to the right in the figure) of the observations taken from Stellwagen Bank. The $x$-coordinate is for reference only, and does not represent actual distances. The upper red curve in the acoustic backscatter is the bottom topography. Other curves seen in the shading likely represent isopycnals, but could also be related to suspended sediment, organisms, or regions of high scalar variance. The backscatter shows two structures that resemble undular bores at $x \approx 35$ and 38 and a depth of $z \approx 25 \mathrm{~m}$. The undular bores appear to deteriorate into shear instabilities and turbulence just downstream. The velocity field indicates that flow separation likely occurs at $x \approx 36$, just below the leading bore. Density profiles, calculated from measured salinity and temperature profiles, on and off the bank are depicted in figure 4-35.

Two-dimensional simulations modeling the flow in Stellwagen bank were conducted to assess how closely the simulations in this chapter agree with existing hydraulic jumps in the environment, and to better understand the flow in Stellwagen bank using the same analyses applied to the more idealized simulations. The simulation setup is very similar to the simulations described in $§ 4.3 .1$. The simulations are nondimensionalized by the total depth away from the topography, giving a characteristic length scale of $\hat{H}=100 \mathrm{~m}$ (Scotti et al., 2007). The top-to-bottom density variation is approximately $\Delta \rho \approx 3.5 \frac{\mathrm{kg}}{\mathrm{m}^{3}}$, with a characteristic density of $\rho_{0}=1023 \frac{\mathrm{kg}}{\mathrm{m}^{3}}$ (figure 4-35

and Pineda, personnal communication). This gives a time scale of $\hat{T} \approx \sqrt{\frac{H}{g^{\prime}}} \approx 55 s$ and a characteristic velocity scale of $\hat{U}=\sqrt{g^{\prime} H}=1.8 \mathrm{~m} / \mathrm{s}$. The volume flow rate per unit width over Stellwagen Bank is $Q=16 \mathrm{~m}^{2} / \mathrm{s}$, or, non-dimensionally, $q=0.087$ (Scotti et al., 2007). The non-dimensional M2 tidal period of $12.42 \mathrm{hr}$ is $T_{T}=820$. 

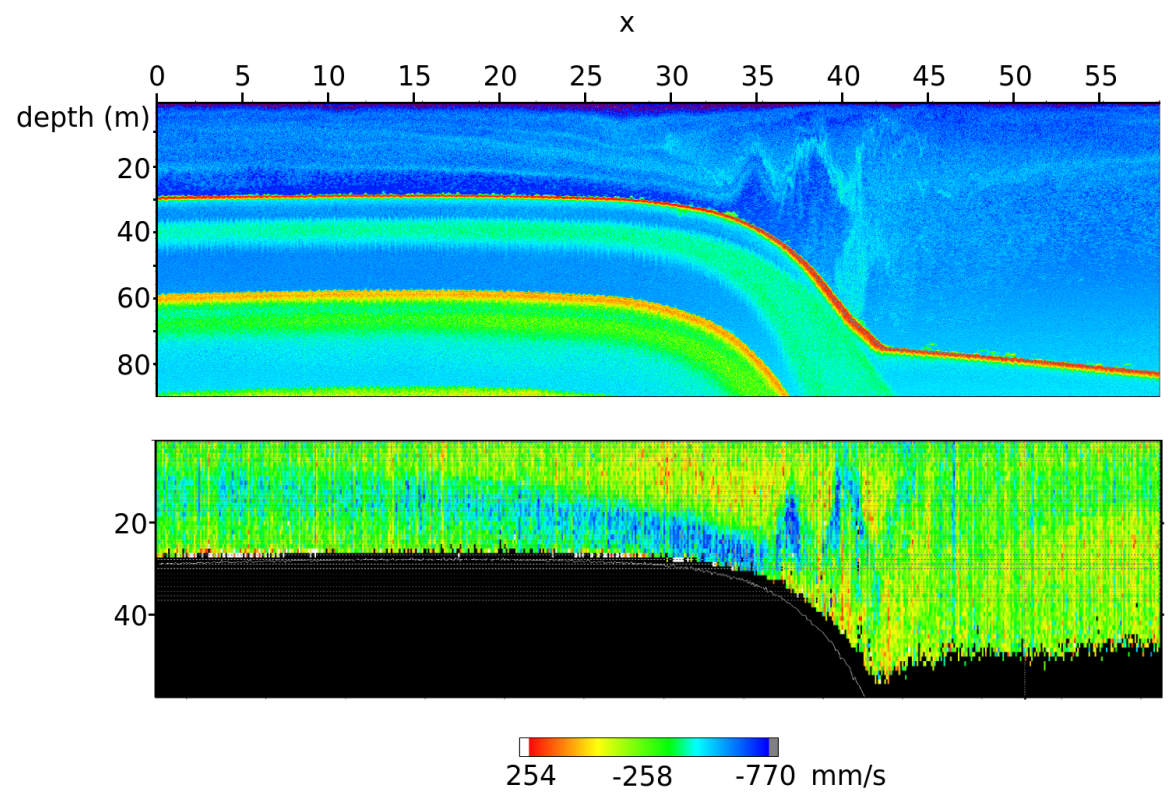

Figure 4-34: Observational data from Pineda (personal communication). (a) Acoustic backscatter and (b) velocity. $x$-coordinate is for reference only, and does not correspond to a physical distance.

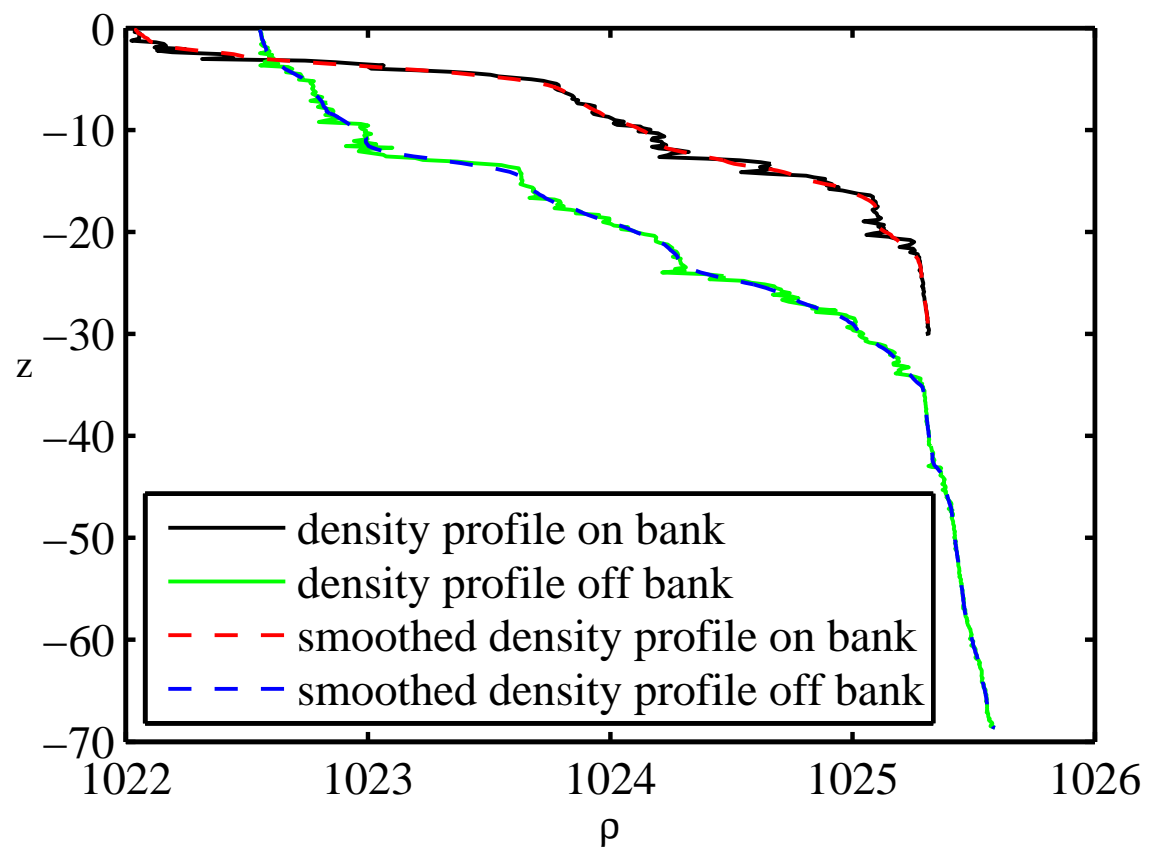

Figure 4-35: Density profiles calculated from observed temperature and salinity profiles (data from Pineda (personal communication, 2016), density calculation done using McDougall and Barker (2011) Oceanographic Toolbox.). 


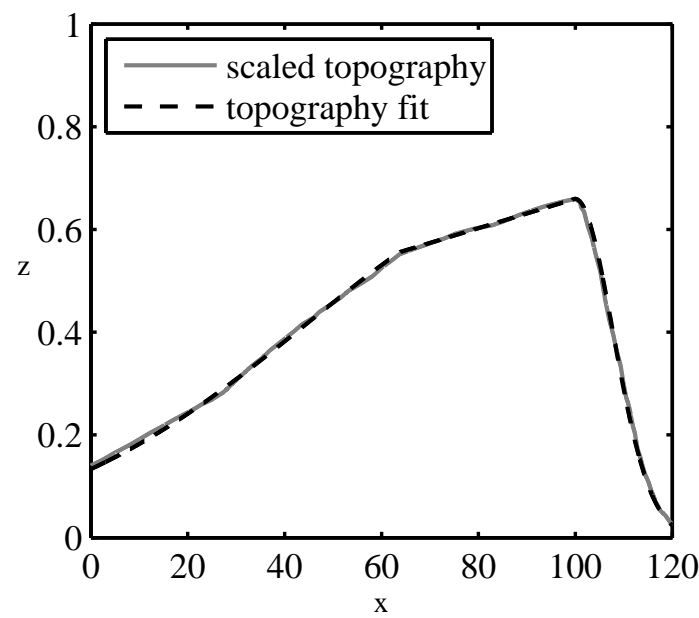

Figure 4-36: Topography from Scotti et al. (2007), non-dimensionalized by $\hat{H}=100 \mathrm{~m}$, and topography fit (4.4.1) used in simulations.

The non-dimensionalized topography used in this simulation is given by:

$$
h_{b}= \begin{cases}0.6596 \times e^{\frac{-(x-63.72)^{2}}{11^{2}}}, & x \geq 100 \\ -0.0029 x+0.7375, & 63.72<x<100 \\ 0.69 \times e^{\frac{-(x-63.72)^{2}}{78^{2}}}, & x<63.72\end{cases}
$$

The expression for the topography (4.4.1) closely fits the topography from Scotti et al. (2007), as shown in figure 4-36, which is non-dimensionalized using the total depth, $\hat{H}=100 \mathrm{~m}$. Bottom-intesified viscosity given by (4.3.6) (figure 4-24) and a no-slip bottom boundary condition are included because the physical topography is used and the observations indicate that the bottom boundary layer separates from the topography, so viscosity and a no-slip bottom boundary condition are required to reproduce this separation. The resolution is 128 grid cells in the vertical away from the topography, with $\Delta x=\Delta z$ constant throughout the domain.

The initial and inlet densities are the smoothed off-bank profile shown in figure 435 , scaled to range from $0 \leq T \leq 1$. The initial velocity field is zero and the flow is 
forced by a non-dimensional inlet velocity given by

$$
U_{\text {inlet }}= \begin{cases}0.1009 \sin \frac{2 \pi t}{T_{T}}, & t \leq \frac{T_{T}}{4} \\ 0.1009, & t>\frac{T_{T}}{4}\end{cases}
$$

which gives a volume flow rate $q=0.087$. Note that the total depth at the inlet is less than one. The velocity increases from zero sinusoidally for a quarter of a tidal period, and then is held at the maximum forcing velocity to allow the jump to fully develop. Time averages and turbulent statistics can then be calculated from the fully developed jump.

Figure 4-37 shows the bottom topography and three other curves taken from the acoustic backscatter, which are assumed to approximate isopycnals. Figure 4-37 also shows the instantaneous density and velocity fields of the simulation at $t=205$, which is at the maximum tidal forcing $\left(T_{T}=820\right)$. Five points from the acoustic backscatter are identified and plotted on the simulation results. Two points are located on the topography and are used to align the observations with the simulation results. The observations are stretched and shifted in the horizontal direction so that the two topography points, whose depths are known, align with the topography in the simulations. This helps place the observed features, such as the undular bore, in the simulated domain. Errors may be introduced by this approach if the ship speed varied while the observations were collected. The other three points help identify the amplitude, wavelength, and location of the leading disturbance in the undular bore.

The simulation produces an undular bore with trailing shear instability that appears to be consistent with the observations. The amplitudes of the first two waves are similar, and both the simulation and the observations show two wave-like undulations before the jet deteriorates into turbulence. The location and wavelength of the bore in the simulation, however, are different from the observations. There are many reasons why the simulations may not match the observations. The topography used in the simulations is likely not exactly the same as the topography in the location of the observations. The density profiles considered are instantaneous profiles from 

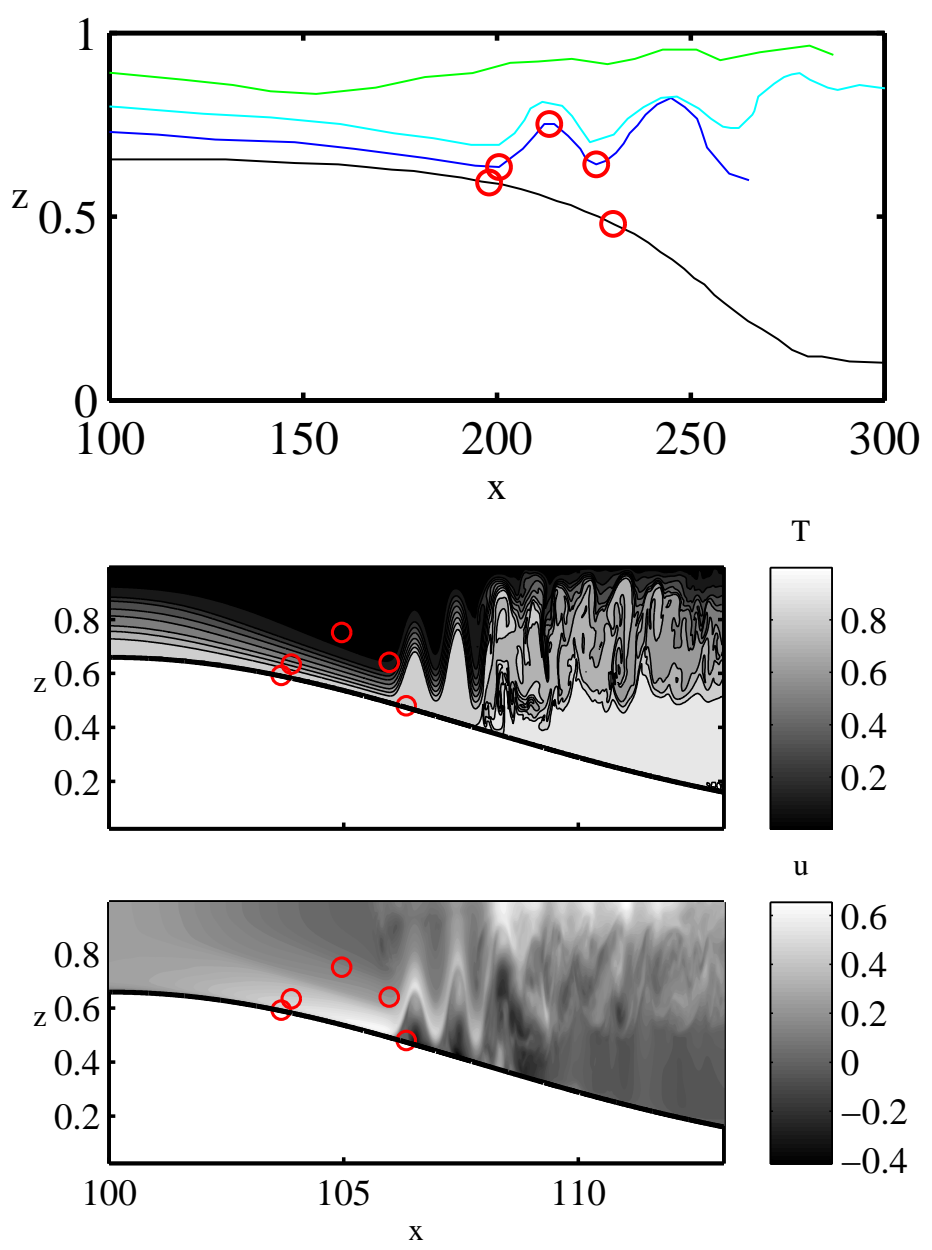

Figure 4-37: (a) Topography and curves from acoustic backscatter, with reference points plotted. Shown before shifting and stretching to align reference points. (b) Density contour plot from simulation with reference points from observational data indicated. (c) Horizontal velocity field from simulation with reference points from observational data indicated. Simulation results shown at $t=\frac{T_{T}}{4}=205$. 
two locations, and are not necessarily the same as the density profile upstream of the sill; the effects of asymmetric density profiles around a sill have been discussed by Lamb (2004) in the context of Knight Inlet, who found the difference to have notable effects on the jump. Furthermore, the previous results in the section indicate that the density profile is important, even to the qualitative jump type. Simulations modifying the topographic height and slope, the tidal period, the volume flow rate, and the viscosity magnitude and profile shape were also compared to the observational data to determine whether modifying any of these parameters produced a flow closer to the observations. The results shown in figure 4-37 are the best match to the observations, and also use the most appropriate parameter values and the off-bank density profile.

This comparison suggests that the qualitative jump types decribed in $\S 4.3 .3$ and the parameter space in which each occurs likely extends to jumps in the environment. The mass balance ratio (figure 4-15) and the scalar variance production (figure 4-19) calculated from the Stellwagen Bank simulation are also consistent with the more idealized simulations of $\S 4.3$, despite the significant differences in background desnity profiles. The hydraulic jump in Stellwagen Bank has a low mass balance ratio of $\frac{M_{a d v, 2}}{\Delta M_{2}}=-0.42$ because a jump with shear instabilities develops, indicating that mixing due to shear instabilities is the main source of intermediate water in the jump in Stellwagen Bank. The scalar variance production is low-to-moderate $(P=-0.0025)$ due to the moderate forcing, and the simulation results are at the lower end of the scatter for the forcing. The results show that jumps in the environment are consistent with the more idealized simulations studied throughout this chapter, and suggest that the conclusions from these simulations can be applied to observations.

\subsection{Conclusions of jumps with topography}

While the two-layer theory can be extended to include topography and the simulation results agree with the theory, the theory is not useful for predicting jumps because the location of the jump must be known in order to specify the upstream conditions. 
Therefore, the theory is set aside and more realistic simulations are used to gain a better understanding of internal hydraulic jumps in the environment.

Simulations show three qualitative jump types that can occur, and the mass budgets and scalar variance production vary with the jump type. Qualitative jump types include mode-one jumps with wave overturning, higher-mode jump with a wedge, and both higher-mode and first-mode jumps with upstream shear instability. The mode of the jump depends on the volume flow rate and less so on topographic slope. Whether the jump has upstream shear instabilities depends on the density profile, particularly the interface thickness, and the downsteam topographic slope. Thin interfaces are unstable and gentle slopes are required for upstream shear instabilities to develop.

Mass budgets show that, at low volume flow rates, mixing plays the main role in producing intermediate water for higher-mode jumps with shear instabilties. However, in higher-mode jumps with a wedge, intermediate-density fluid comes from net advection in from upstream. The first-mode jumps have an initial time period during which advection is significant, but mixing becomes the main source of intermediatedensity fluid when wave overturning begins or upstream shear instabilities develop. Jumps with wave overturning and jumps with upstream shear instabilities have similar mass budgets, despite the different types of mixing.

Mixing efficiencies for $2 \mathrm{D}$ jumps are similar to those found in chapter $2\left(R i_{f} \approx 0.5\right)$. Scalar variance production is studied to identifiy how varying parameters affects the amount of mixing in a jump. Scalar variance production depends primarily on upstream shear, which develop as the flow spills over the sill. Scalar variance production increases with increasing upstream shear, which depends mainly on $q$, and less so on $h_{T}$. Other parameters, such as $T_{T}$ and $\nu$ do not have a significant effect on the scalar variance production.

Most of the simulations in this chapter have a free-slip bottom boundary and no explicit viscosity. In this case, the bottom topography can be considered 'modified' to 
follow the path that the jet of separated fluid would follow is bottom boundary layer separation occured. However, several simulations with a no-slip bottom boundary and bottom ntensified viscosity are conducted to determine if the mixing trends and qualitative jump types change. The results show that the same jump types develop and mass budgets and mixing trends remain the same. However, the simulations with viscosity do show that in some cases, viscosity can cause flow separation that mimics a gentler topographic slope and results in a jump with upstream shear instabilities when wave overturing would otherwise occur. This gentler slope is simulated by using modified topography in simulations without viscosity. Furthermore, even in cases in which the jump type does not change, the structure of the jump has small changes due to instabilities on the under-side of the jet, and the velocity field changes significantly due to flow separation.

Jumps in $3 \mathrm{D}$ are very similar to $2 \mathrm{D}$ jumps, with lower $R i_{f}$ and scalar variance production. Otherwise, jumps are qualitatively very similar, with similar mass budgets. This result is consistent with chapter 2 , which is expected because these jumps have similar upstream shear values.

The flow in Stellwagen Bank is simulated using observed density profiles and topography, and the results are compared to observational data (Pineda, 2016). Some features of the simulated flow, such as the wavelength and position of the udular bores, are different. However, the jump structure of an undular bore with two waves followed by turbulence, and the amplitude of the undulations, are reproduced fairly accurately. The mixing and mass budget of the Stellwagen Bank simulation also follow the same trends as the other, more idealized simulations described in this chapter. This suggests that the conclusions drawn here could help understand jumps found in the environment. 


\section{Chapter 5}

\section{Conclusions}

\subsection{Theory}

This work investigates internal hydraulic jumps using two-layer theories and numerical simulations. Several two-layer theories are extended to include upstream shear, and the solution space is identified. These theories require assuming that the energy flux is conserved in either the upper or lower layer, or apply vorticity conservation. As upstream shear increases, the basic two-layer theories do not have solutions, and entrainment and consideration of continuous velocity profiles are required for the theory to be consistent with simulations. The entrainment can be predicted as part of the solution by relating it to the square upstream shear. This relationship suggests that, as upstream shear increases, the jumps behave in some cases similar to an expanding shear layer. The predictive value of the two-layer theory is also limited for high shear flows due to the importance of continuous density and velocity profiles, which are quantified in the theory by shape parameters. While the theory indicates the physical processes that are important in this type of jump, it is not very helpful in predicting how a jump will behave. Topography can also be incorporated into the two-layer theory by allowing a change in total depth across the jump and including the form drag on the topography. While this modification provides predictions that are broadly consistent with the simulations, the theory is again not useful for predicting jumps because the location of the jump must be known a priori to identify the upstream parameters. 
The theoretical aspects of this thesis focus on the approaches of Wood and Simpson (1984) and Klemp et al. (1997), who close the equation set by making an assumption about where dissipation occurs in the jump. Recent work by Borden and Meiburg (2013b), who consider the mean vorticity budget instead of the momentum flux equation, avoid such an assumption, and their approach is also considered here and extended to include upstream shear and topography. However, the vorticity budgets of the high shear jumps with entrainment show that the turbulent vorticity fluxes are of leading order, so mean vorticity conservation is not applicable in those flows. While the vorticity approach is useful and cleverly avoids assumptions about the distribution of dissipation, it is limited as the flows become intensely turbulent.

This work with two-layer theories provides useful insight into the behavior of hydraulic jumps in finite-depth flows with upstream shear and demonstrates that simplified theories are useful for the physical insights that they can provide. However, this work also illustrates the limitations of such simplified theories, in particular the limitations of the two-layer approximation. The two-layer approximation works well for flows that are approximately two-layered upstream of the jump and have low upstream shear. However, as shear increases and entrainment becomes important, the continuous velocity profiles must be taken into account to accurately describe the flow because the shape of the profiles deviate significantly from two-layered and from the shape of a classic shear layer, with a velocity minimum developing in some cases.

In hydraulic jumps in the environment, the downstream conditions affect jump properties by influencing the location and height of the jump. As a jump develops, there is complicated feedback between the upstream flow over topography, the developing jump, the downstream topography or stratification, and possibly upstream adjustment of the flow. The problem of super-critial flow over topography with a downstream sill was recently investigated by Winters (2016). In the theories developed in this work, it is assumed that the upstream parameters are known, which limits the applicability of the theories to flows over topography, and is one reason the 
theories are abandoned after $\S 4.2 .3$. However, the theory could be modified to include the downstream influence. An example of this approach is the work of Wilkinson and Wood (1971), which is adapted to the two-layer problem with large upstream shear in §3.4.1. This method failed because the flow was not necessarily critical at the crest of the sill, as the theory assumed. This approach may provide better predictions in lower shear flows, although that has not been investigated here. The downstream influence could be added to the theory for a jump over topography by assuming the position of the interface far downstream of the jump and searching (through iteration) for a jump position and height that matches the downstream interface height and the upstream conditions. However, this theory would still be limited by the two-layer approximation, which is often inconsistent with the downstream density and velocity profiles.

Current theoretical work on internal hydraulic jumps is moving toward theories with continuous stratification (Thorpe, 2010; White and Helfrich, 2014; Nasr-Azadani and Meiburg, 2016), often taking the vorticity approach. Nasr-Azadani and Meiburg (2016) use vorticity conservation to derive a equation for the displacement of isopycnals for a continuously stratified and sheared ambient with a propagating gravity current. Their work is similar to the wave treatment of internal hydraulic jumps discussed in $\S 2.3 .3$, and applies to gravity current with smooth fronts. Lin and Redekopp (2012) also study internal hydraulic jumps in a continuously stratified and sheared flow by applying conservation laws to streamtubes. They show that upstream shear allows solutions for a greater range of parameters and jumps of larger amplitude, consistent with the conclusions of chapter 2 . This type work will likely provide insights into the physics of these jumps. However, it will also likely be limited by the need to assume the shape of the density and velocity profiles downstream of the jump, to which this work demonstrated that the theory was highly sensitive, or to the assumption of a smooth front, which would exclude the fully turbulent jumps found throughout this work. 


\subsection{Simulations: jump types}

Numerical simulations, which are compared to the two-layer theories throughout most of this work, also provide additional insight into the behavior of internal hydraulic jumps. The simulations reveal the many qualitative jump types that may occur, and under what conditions each type will develop. At low upstream shears, jumps may be undular bores (low shear and small lower layer velocities), smooth front turbulent jumps (low shear and moderate lower layer velocities), fully turbulent jumps (moderate shears), or conjugate state jumps (a special energy conserving solution at low shear that gives a large jump). As upstream shear increases, jumps begin to behave as an expanding shear layer, with a longer jump transition including either an entrainment region and a roller region for super- to sub-critical jumps or an entrainment region for super- to super-critical jumps. When the jump occurs over topography, additional jumps structures are possible because the topography provides a frame of reference from which to analyze a higher-mode jump. This allows higher-mode wedges to develop. Additionally, jumps with wave overturning, which are similar to the fully turbulent jump, and jumps with upstream shear instabilities, which are similar to the high shear jump, are both possible.

This work mainly focuses on very idealized jumps, moving toward more realistic jumps by including topography and some time dependence in chapter 4 . The simulations include approximately two-layered density stratifications, which is a reasonable approximation for the internal hydraulic jumps in exchange flows, and also allows the reproduction of qualitative features seen in tidally driven flows, such as a wedge, overturning wave, and jumps with upstream shear instability. A simulation reproducing the flow in Stellwagen Bank, using an observed density profile, is also consistent with the idealized two-layered simulations. The jump structure in the simulation matches the jump structure seen in the observations as well as the jumps structures seen in more idealized simulations with similar parameters. This suggests that the jump structure categorization based on the idealized simulations can help predict the jump types that might occur in hydraulic jumps in the environment. 
This extends existing idealized work, which commonly applies a constant density stratification (Winters and Armi, 2012, 2013), and helps close the gap between very idealized simulations and the very realistic simulations of specific locations such as Knight Inlet (Lamb, 2004; Stashchuk and Vlasenko, 2007).

\subsection{Simulations: mixing}

Identifying mixing trends is one on the main goals of this work. Mixing in internal hydraulic jumps is important because of the energy that it can dissipate and the changes in water properties that result. At low shear, the mixing efficiency for $2 \mathrm{D}$ simulations is $R i_{f} \approx 0.5$, and for $3 \mathrm{D}$ simulations $R i_{f} \approx 0.2$. As upstream shear increases and entrainment becomes important, the mixing efficiency decreases, asymptoting toward $R i_{f} \rightarrow 0$. This transition occurs in the same range of shear values, $3 \lesssim s \lesssim 8$, through which the basic two-layer theories lose solutions and entrainment becomes necessary to predict two-layer jumps. Flows over topography tend to develop low upstream shear values, so the $2 \mathrm{D}$ mixing efficiencies are similar to the values found for the very idealized jumps with low upstream shear. These jumps over topography are more realistic than the idealized simulations with a flat bottom. This shows that the idealized work can be used to help understand more complicated flow in the environment. An alternative metric of mixing, the cumulative integral of the scalar (density) variance production, varies between jumps even when the mixing efficiency is approximately constant. In jumps with topography, it was found to increase with the upstream shear, which illustrates the important influence that upstream shear has on the jump, even at low shear values.

There has been significant discussion in the literature about whether wave overturning or upstream shear instability generates the intermediate density water in internal jumps, with many of the studies focusing on Knight Inlet (Lamb, 2004;

Farmer and Armi, 1999). Simulations with topography demonstrate that mixing can 
be initiated by either wave overturning or upstream shear instability, depending on the parameters of the flow. Which of the two occurs depends primarily on the density profile and the downstream topographic slope. Although the results do not provide direct evidence of whether wave overturning or upstream shear instabilities cause the mixing in specific places such as Knight Inlet, the results show that either mechanism is possbile. They also demonstrate that bottom boundary layer separation can cause upstream shear instability in flows that would otherwise not experience this by creating an effectively gentler slope and allowing the instabilties enough space to develop. However, for a given volume flow rate, the mass budgets and the scalar variance production generated by both types are very similar.

\subsection{Applications}

The results of chapter 4 are the most relevant in understanding internal hydraulic jumps in the environment. Many jump properties depend on the vertical structure of the density profile, the details of which determine whether upstream shear instabilities will develop. If the volume flow rate is low, the existence of upstream shear instabilities has a significant impact on the mass balance ratio $\frac{M_{a d v, 2}}{\Delta M_{2}}$. At higher volume flow rates, when the possible jumps types are wave overturning or first-mode jumps with upstream shear instabilities, the mass balance ratio is not highly dependent on the jump type. In this case, simply knowing the volume flow rate allows an estimate of the mass balance ratio. The scalar variance production follows the same trend for all of the density profiles considered in this thesis, including the observed profile from Stellwagen Bank. The regime diagrams in figure 4-23 can therefore be used to estimate the scalar variance production of jumps in the environment. The amount of upstream shear that will develop, the mode and qualitative jump type, and the source of intermediate water could also be estimated using figures 4-23, 410, 4-11, 4-12, and 4-15. Since the density profile can have a significant influence on the jump properties, particularly the jump structure and source of intermediate water, the accuracy of predictions will depend on the similarity of the observed pro- 
files to the idealized profiles studied here. However, the simulations of Stellwagen Bank described in $\S 4.4$ indicate that the trends found in idealized simulations may be reasonably consistent with observed jumps.

This thesis focuses on jumps in peripheral channels and the coastal ocean, where friction is not significant (table 1.2). However, there are dense overflows in the deep ocean that might also be expected to exhibit hydraulic jumps, such as in the Faroe Bank Channel and the Denmark Strait. However, in these locations, significant entrainment occurs without a sudden jump. This is likely because the topographic slope is gentle and friction is therefore an important consideration. Also, the gentle topographic slope would allow the flow to develop and entrain overlying fluid slowly, without requiring a jump to quickly transition to a downstream state. Furthermore, rotation may be important in these larger scale jumps, and the effect of rotation has not been studied here.

Hydraulically controlled flow over topography results in form drag by the topography on the flow due to the asymmetry of the flow and resulting pressure field. The form drag due to a hydraulically controlled flow that includes a hydraulic jump is important when considering the momentum balance of flows in a larger geographical region. If the location of the jump and the jump height are known, the hydrostatic pressure can be calculated and used to estimate the form drag. To estimate the jump height, the theory that includes a change in total depth across the jump (either the KRS/WS theory or the VS theory with topography, §4.2) could be used, if the jump location is known. This approach involves the same difficulties discussed in $§ 4.2 .3$ because the jump location may not be known. Furthermore, only the hydrostatic pressure can be included when using the theory. This approach neglects the nonhydrostatic pressure and the finite length the jump head.

The momentum balance of the Stellwagen Bank simulation identifies the signifi- 
cance of the form drag. The momentum flux balance is

$$
\left[\int_{h_{b}}^{D}\left(p_{\text {hydros }}+p_{\text {non-hydros }}+\rho u^{2}\right) d z\right]_{b}^{a}+\oint_{h_{b}} p \cdot \hat{n} d s=\oint_{h_{b}} D_{\text {friction }} d s
$$

where the first term is the net momentum flux due to the flow, the second term is the form drag, and the term on the right hand side is the frictional drag. The leading order balance is between the difference of the vertical integrals of the upstream and downstream non-hydrostatic pressure terms (0.11) and the form drag $(-0.08)$, which are an order of magnitude larger than the velocity flux term (-0.02), the vertical integrals of the upstream and downstream hydrostatic pressure (0.01) and the frictional drag (0.02). This shows that the form drag resulting from an internal hydraulic jump is a significant term in the momentum flux balance. Using the theory and assuming the pressure is hydrostatic to calculate the form drag $\left(D_{\text {form }}=\oint_{h_{b}} p \cdot \hat{n} d s=-\left[\int_{h_{b}}^{D}\left(p_{\text {hydros }}+\rho u^{2}\right) d z\right]_{a}^{b}\right)$ will not provide a good estimate because it neglects the leading order term $\left[\int_{h_{b}}^{D} p_{\text {non-hydros }} d z\right]_{a}^{b}$.

\subsection{Future work}

Many questions about the physics of internal hydraulic jumps remain. Some results from this thesis could be further explored, such as what determines the direction of entrainment. In jumps with very large upstream shear, the entrainment is large and into the lower (active) layer. However, in jumps with topography, entrainment is often negative, with a small to moderate magnitude. An explanation for why the entrainment changes sign, and for what parameter values, has not been provided. Based on the results of this thesis, the answer is likely related to the height and perhaps slope of the topography and to the upstream shear, which is often controlled by the topography. Furthermore, the definition of entrainment used in this work assumes that the flow is two-layered, which can be a poor assumption, particularly for high shear flows. A more extensive description of entrainment in internal hydraulic jumps may require redefining the term to accomodate continuous density profiles. 
Three-dimensional simulations have also been conducted sparingly in this work due to high computational costs. Although several 3D simulations have shown that many of the results described in this work carry over to 3D, more extensive simulations would be beneficial, particularly in studying high shear flows, which exhibit significant differences in 3D compared to 2D. 3D simulations will also be necessary to study the effects of two-dimensional topography.

Finally, many other factors that affect internal hydraulic jumps in the environment have not been considered in this work. These include rotation, which could be important in locations such as Hood Canal (Gregg and Pratt, 2010) and the Strait of Gibraltar (Sánchez-Garrido et al., 2011), and two-dimensional topography including contractions and non-rectangular cross-sections, which has been identified as important in Hood Canal (Gregg and Pratt, 2010). 


\section{Appendix A}

\section{Calculating Entrainment with Controlled Flow at Weir}

In this section, equations are derived to calculate the jump height and entrainment by assuming that the flow is controlled at the weir and energy is conserved between the region downstream of the jump and the weir. This follows the work of Wilkinson and Wood (1971), who applied the concept to a one-and-a-half layer flow with a stagnant upper layer. It is applied here to a two-layer flow whose upper layer can have a velocity independent of the lower layer. Figure 3-25b depicts the flow setup. Variables are defined in figure 2-1.

The pressure through the depth of the flow is given by

$$
p=p_{s}+\int_{y}^{D} \rho g d y
$$

Let $\rho=\rho_{0}+\Delta \rho$, where $\rho_{0}=\min (\rho)=\rho_{2}$.

Then, the pressure is

$$
p=p_{s}+\int_{y}^{D} \rho_{0} g d y+\int_{y}^{D} \Delta \rho g d y=p_{s}+\rho_{0} g(D-y)+\int_{y}^{D} \Delta \rho g d y .
$$


The mass flux $\left(q_{M}\right)$ in the lower layer is defined as

$$
\frac{q_{M}}{\rho_{0}}=\int_{0}^{d_{1}} \frac{\rho}{\rho_{0}} u d y=\int_{0}^{d_{1}} \frac{\left(\rho_{0}+\Delta \rho\right)}{\rho_{0}} u d y=\int_{0}^{d_{1}} u d y+\int_{0}^{d_{1}} \frac{\Delta \rho}{\rho_{0}} u d y
$$

The mass flux can then be re-written in terms of the volume flux $\left(q_{L}=\int_{0}^{d_{1}} u d y\right)$ :

$$
\frac{q_{M}}{\rho_{0}}=\int_{0}^{d_{1}} u d y+\int_{0}^{d_{1}} \frac{\Delta \rho}{\rho_{0}} u d y=q_{L}+\frac{q_{\Delta}}{g} .
$$

Since $q_{M}$ and $q_{L}$ are conserved, $q_{\Delta}$ must also be conserved. The same concept applies to the upper layer, and these results will be used later.

\section{A.1 Lower Layer}

Energy flux, $q_{E}$, is conserved in each layer away from the jump because the flow is frictionless and the interface is a streamline. Therefore, energy flux is conserved along the interface and no energy crosses the interface. In the lower layer,

$$
\frac{q_{E}}{\rho_{0}}=\int_{0}^{d_{1}}\left(\frac{p}{\rho_{0}}+\frac{u^{2} \rho}{2 \rho_{0}}+\frac{\rho}{\rho_{0}} g\left(y+h_{b}\right)\right) u d y .
$$

The flow is Boussinesq, so

$$
\frac{q_{E}}{\rho_{0}}=\int_{0}^{d_{1}}\left(\frac{p}{\rho_{0}}+\frac{u^{2}}{2}+\frac{\rho}{\rho_{0}} g\left(y+h_{b}\right)\right) u d y .
$$

Substitute (A.0.1) for the pressure in to (A.1.1) to get

$$
\frac{q_{E}}{\rho_{0}}=\int_{0}^{d_{1}}\left(\frac{p_{s}+\rho_{0} g(D-y)+\int_{y}^{D} \Delta \rho g d y}{\rho_{0}}+\frac{u^{2}}{2}+\frac{\rho}{\rho_{0}} g\left(y+h_{b}\right)\right) u d y .
$$

The equation can be re-written as follows:

$$
\frac{q_{E}}{\rho_{0}}=\int_{0}^{d_{1}}\left(\frac{p_{s}}{\rho_{0}}+g(D-y)+\int_{y}^{D} \frac{\Delta \rho}{\rho_{0}} g d y+\frac{u^{2}}{2}+\frac{\rho_{0}+\Delta \rho}{\rho_{0}} g\left(y+h_{b}\right)\right) u d y
$$




$$
\begin{gathered}
\frac{q_{E}}{\rho_{0}}=\int_{0}^{d_{1}}\left(\frac{p_{s}}{\rho_{0}}+g(D-y)+\int_{y}^{D} \frac{\Delta \rho}{\rho_{0}} g d y+\frac{u^{2}}{2}+\frac{\Delta \rho}{\rho_{0}} g\left(y+h_{b}\right)+g\left(y+h_{b}\right)\right) u d y \\
\frac{q_{E}}{\rho_{0}}=\int_{0}^{d_{1}}\left(\frac{p_{s}}{\rho_{0}}+g\left(D+h_{b}\right)+\int_{y}^{D} \frac{\Delta \rho}{\rho_{0}} g d y+\frac{u^{2}}{2}+\frac{\Delta \rho}{\rho_{0}} g\left(y+h_{b}\right)\right) u d y \\
\frac{q_{E}}{\rho_{0}}=q_{L}\left(\frac{p_{s}}{\rho_{0}}+g\left(D+h_{b}\right)\right)+\int_{0}^{d_{1}}\left(\int_{y}^{D} \frac{\Delta \rho}{\rho_{0}} g d y+\frac{u^{2}}{2}+\frac{\Delta \rho}{\rho_{0}} g\left(y+h_{b}\right)\right) u d y \\
\frac{q_{E}}{\rho_{0}}=q_{L}\left(\frac{p_{s}}{\rho_{0}}+g\left(D+h_{b}\right)\right)+\int_{0}^{d_{1}}\left(\int_{y}^{D} \frac{\Delta \rho}{\rho_{0}} g d y+\frac{\Delta \rho}{\rho_{0}} g y+\frac{u^{2}}{2}\right) u d y+h_{b} q_{\Delta} \\
\frac{q_{E}}{\rho_{0}}=q_{L}\left(\frac{p_{s}}{\rho_{0}}+g\left(D+h_{b}\right)\right)+\int_{0}^{d_{1}}\left(\int_{y}^{D} \Delta \rho d y+\Delta \rho y\right) \frac{g u}{\rho_{0}} d y+\int_{0}^{d_{1}} \frac{u^{3}}{2} d y+h_{b} q_{\Delta}
\end{gathered}
$$

Non-dimensional quantities are defined as follows:

$$
\hat{u}=\frac{u d_{1}}{q_{L}} ; \quad \hat{\Delta}=\frac{\frac{\Delta \rho}{\rho_{0}} g}{\frac{q_{\Delta}}{q_{L}}} ; \hat{y}=\frac{y}{d_{1}}
$$

Shape parameters are defined as follows:

$$
S_{E}=\int_{0}^{1} \hat{u}^{3} d \hat{y} ; \quad S_{P}=\int_{0}^{1} \hat{u}\left(\int_{\frac{y}{d_{1}}}^{\frac{D}{d_{1}}} \hat{\Delta} d \hat{y}+\hat{\Delta} \hat{y}\right) d \hat{y}
$$

Using the non-dimensional variables (A.1.3) and the shape parameters (A.1.4) in the energy flux conservation equations (A.1.2) gives

$$
\frac{q_{E}}{\rho_{0}}=q_{L}\left(\frac{p_{s}}{\rho_{0}}+g\left(D+h_{b}\right)\right)+h_{b} q_{\Delta}+\frac{q_{L}^{3}}{d_{1}^{2}} \frac{S_{E}}{2}+q_{\Delta} d_{1} S_{P} .
$$

Shape functions are assumed not to vary along the flow, except across the jump. $d_{1}$ and $p_{s}$ vary along the channel, forced by the imposed along channel variation of $h$. 
Equation (A.1.5) can be re-written as

$$
\frac{q_{E}}{\rho_{0}}-q_{L} g\left(D+h_{b}\right)=q_{L} \frac{p_{s}}{\rho_{0}}+h_{b} q_{\Delta}+\frac{q_{L}^{3}}{d_{1}^{2}} \frac{S_{E}}{2}+q_{\Delta} d_{1} S_{P}
$$

where the left hand side is constant along the channel and the right hand side varies as $h$ varies. Differencing the equation between two points that do not bracket a hydraulic jump the gives

$$
\left[q_{L} \frac{p_{s}}{\rho_{0}}+h_{b} q_{\Delta}+\frac{q_{L}^{3}}{d_{1}^{2}} \frac{S_{E}}{2}+q_{\Delta} d_{1} S_{P}\right]_{b}^{e}=0
$$

which applies between the region downstream of the jump and the point at the crest of the downstream sill.

The flow is assumed to be controlled at the crest of the sill. Equation ( A.1.6) is differentiated with respect to $x$, and at a critical point, $\frac{d h_{b}}{d x}=0$ and $\frac{d d_{1}}{d x} \neq 0$. This gives

$$
0=\frac{q_{L}}{\rho_{0}} \frac{d p_{s}}{d x}-2 \frac{q_{L}^{3}}{d_{1}^{3}} \frac{S_{E}}{2} \frac{d d_{1}}{d x}+q_{\Delta} S_{P} \frac{d d_{1}}{d x} .
$$

Noting that $F_{1}^{2}=\frac{q_{L}^{3}}{d_{1}^{3} q_{\Delta}}$ and substituting this into (A.1.8) gives

$$
0=\frac{q_{L}}{\rho_{0} q_{\Delta}} \frac{d p_{s}}{d x}-F_{1}^{2} S_{E} \frac{d d_{1}}{d x}+S_{P} \frac{d d_{1}}{d x}
$$

\section{A.2 Upper layer}

Now consider the energy conservation in the upper layer. The same process is followed as in $\S$ A.1, and the equations are summarized here.

$$
\frac{q_{E}}{\rho_{0}}=\int_{d_{1}}^{D}\left(\frac{p}{\rho_{0}}+\frac{u^{2} \rho}{2 \rho_{0}}+\frac{\rho}{\rho_{0}} g\left(y+h_{b}+d_{1}\right)\right) u d y
$$

The flow is Boussinesq:

$$
\frac{q_{E}}{\rho_{0}}=\int_{d_{1}}^{D}\left(\frac{p}{\rho_{0}}+\frac{u^{2}}{2}+\frac{\rho}{\rho_{0}} g\left(y+h_{b}+d_{1}\right)\right) u d y
$$


Substitute for the pressure (note that the limits on the pressure integral change so that $\left.p=p_{s}+\rho_{0} g\left(D-\left(y+d_{1}\right)\right)+\int_{y}^{D} \Delta \rho g d y\right)$ :

$$
\begin{gathered}
\frac{q_{E}}{\rho_{0}}=\int_{d_{1}}^{D}\left(\frac{p_{s}+\rho_{0} g\left(D-\left(y+d_{1}\right)\right)+\int_{y}^{D} \Delta \rho g d y}{\rho_{0}}+\frac{u^{2}}{2}+\frac{\rho}{\rho_{0}} g\left(y+h_{b}+d_{1}\right)\right) u d y \\
\frac{q_{E}}{\rho_{0}}=\int_{d_{1}}^{D}\left(\frac{p_{s}}{\rho_{0}}+g\left(D-y-d_{1}\right)+\int_{y}^{D} \frac{\Delta \rho}{\rho_{0}} g d y+\frac{u^{2}}{2}+\frac{\rho_{0}+\Delta \rho}{\rho_{0}} g\left(y+h_{b}+d_{1}\right)\right) u d y \\
\frac{q_{E}}{\rho_{0}}=\int_{d_{1}}^{D}\left(\frac{p_{s}}{\rho_{0}}+g\left(D-y-d_{1}\right)+\int_{y}^{D} \frac{\Delta \rho}{\rho_{0}} g d y+\frac{u^{2}}{2}+\frac{\Delta \rho}{\rho_{0}} g\left(y+h_{b}+d_{1}\right)+\frac{\Delta \rho}{\rho_{0}} g\left(y+h_{b}+d_{1}\right)\right) u d y
\end{gathered}
$$

$$
\frac{q_{E}}{\rho_{0}}=\int_{d_{1}}^{D}\left(\frac{p_{s}}{\rho_{0}}+g\left(D+h_{b}\right)+\int_{y}^{D} \frac{\Delta \rho}{\rho_{0}} g d y+\frac{u^{2}}{2}+\frac{\Delta \rho}{\rho_{0}} g\left(y+h_{b}+d_{1}\right)\right) u d y
$$

$$
\frac{q_{E}}{\rho_{0}}=q_{U}\left(\frac{p_{s}}{\rho_{0}}+g\left(D+h_{b}\right)\right)+\int_{d_{1}}^{D}\left(\int_{y}^{D} \frac{\Delta \rho}{\rho_{0}} g d y+\frac{u^{2}}{2}+\frac{\Delta \rho}{\rho_{0}} g\left(y+h_{b}+d_{1}\right)\right) u d y,
$$

where $q_{U}=\int_{0}^{D} u d y$. Note that 0 is located at the interface, so $q_{U}$ is the volume flux in the upper layer. Continued rearrangement gives

$$
\begin{aligned}
& \frac{q_{E}}{\rho_{0}}=q_{U}\left(\frac{p_{s}}{\rho_{0}}+g\left(D+h_{b}\right)\right)+\int_{0}^{D}\left(\int_{y}^{D} \frac{\Delta \rho}{\rho_{0}} g d y+\frac{\Delta \rho}{\rho_{0}} g y+\frac{u^{2}}{2}\right) u d y+\left(h_{b}+d_{1}\right) q_{\Delta U}, \\
& \frac{q_{E}}{\rho_{0}}=q_{U}\left(\frac{p_{s}}{\rho_{0}}+g\left(D+h_{b}\right)\right)+\int_{0}^{D}\left(\int_{y}^{D} \Delta \rho d y+\Delta \rho y\right) \frac{g u}{\rho_{0}} d y+\int_{0}^{D} \frac{u^{3}}{2} d y+\left(h_{b}+d_{1}\right) q_{\Delta} .
\end{aligned}
$$

Non-dimensional variables are 


$$
\hat{u}=\frac{u d_{2}}{q_{U}} ; \quad \hat{\Delta}=\frac{\frac{\Delta \rho}{\rho_{0}} g}{\frac{q_{\Delta U}}{q_{U}}} ; \hat{y}=\frac{y}{d_{2}} .
$$

Shape parameters for the upper layer are

$$
S_{E U}=\int_{d_{1}}^{\frac{D}{d_{2}}} \hat{u}^{3} d \hat{y} ; \quad S_{P U}=\int_{d_{1}}^{\frac{D}{d_{2}}} \hat{u}\left(\int_{\frac{y}{d_{2}}}^{\frac{D}{d_{2}}} \hat{\Delta} d \hat{y}+\hat{\Delta} \hat{y}\right) d \hat{y}
$$

Using the above definitions in energy flux conservation gives

$$
\frac{q_{E U}}{\rho_{0}}=q_{U}\left(\frac{p_{s}}{\rho_{0}}+g\left(D+h_{b}\right)\right)+\left(h_{b}+d_{1}\right) q_{\Delta U}+\frac{q_{U}^{3}}{d_{2}^{2}} \frac{S_{E U}}{2}+q_{\Delta U} d_{2} S_{P U} .
$$

Equation (A.2.1) can be arranged so that the left hand side is constant along the channel and the right hand side varies as $h$ varies. This gives

$$
\frac{q_{E U}}{\rho_{0}}-q_{U} g\left(D+h_{b}\right)=q_{U} \frac{p_{s}}{\rho_{0}}+\left(h_{b}+d_{1}\right) q_{\Delta U}+\frac{q_{U}^{3}}{d_{2}^{2}} \frac{S_{E U}}{2}+q_{\Delta U} d_{2} S_{P U} .
$$

Differencing (A.2.2) between the region downstream of the jump and the point at the sill crest gives

$$
\left[q_{U} \frac{p_{s}}{\rho_{0}}+\left(h_{b}+d_{1}\right) q_{\Delta U}+\frac{q_{U}^{3}}{d_{2}^{2}} \frac{S_{E U}}{2}+q_{\Delta U} d_{2} S_{P U}\right]_{b}^{e}=0
$$

if points $\mathrm{b}$ and e do not bracket a hydraulic jump.

Differentiating (A.2.2) with respect to $x$ and considering a critical point (where $\frac{d h}{d x}=0$ and $\left.\frac{d d_{1}}{d x} \neq 0\right)$ gives

$$
0=\frac{q_{U}}{\rho_{0}} \frac{d p_{s}}{d x}+q_{\Delta U} \frac{d d_{1}}{d x}-2 \frac{q_{U}^{3}}{d_{2}^{3}} \frac{S_{E U}}{2} \frac{d d_{2}}{d x}+q_{\Delta U} S_{P U} \frac{d d_{2}}{d x}
$$

Noting that $F_{2}^{2}=\frac{q_{U}^{3}}{h_{U}^{3} q_{\Delta U}},(\mathrm{~A} .2 .4)$ becomes

$$
0=\frac{1}{q_{\Delta U}}\left(\frac{q_{U}}{\rho_{0}} \frac{d p_{s}}{d x}+q_{\Delta U} \frac{d d_{1}}{d x}\right)-2 F_{2}^{2} \frac{S_{E U}}{2} \frac{d d_{2}}{d x}+S_{P U} \frac{d d_{2}}{d x},
$$

and since $d_{2}=D-d_{1}-h_{b}$, this gives 


$$
0=\frac{1}{q_{\Delta U}}\left(\frac{q_{U}}{\rho_{0}} \frac{d p_{s}}{d x}+q_{\Delta U} \frac{d d_{1}}{d x}\right)-2 F_{2}^{2} \frac{S_{E U}}{2} \frac{d\left(D-d_{1}-h_{b}\right)}{d x}+S_{P U} \frac{d\left(D-d_{1}-h_{b}\right)}{d x}
$$

For critical flow, $\frac{d h_{b}}{d x}=0$. Also, $D$ is constant, giving

$$
0=\frac{1}{q_{\Delta U}}\left(\frac{q_{U}}{\rho_{0}} \frac{d p_{s}}{d x}+q_{\Delta U} \frac{d d_{1}}{d x}\right)+F_{2}^{2} S_{E U} \frac{d d_{1}}{d x}-S_{P U} \frac{d d_{1}}{d x}
$$

or

$$
0=\frac{q_{U}}{q_{\Delta U} \rho_{0}} \frac{d p_{s}}{d x}+\frac{d d_{1}}{d x}+F_{2}^{2} S_{E U} \frac{d d_{1}}{d x}-S_{P U} \frac{d d_{1}}{d x}
$$

\section{A.3 Combining upper and lower layer equations to find $h_{1 e}$}

The lower layer at the weir satisfies

$$
0=\frac{q_{L}}{\rho_{0}} \frac{d p_{s}}{d x}-2 \frac{q_{L}^{3}}{d_{1}^{3}} \frac{S_{E}}{2} \frac{d d_{1}}{d x}+q_{\Delta} S_{P} \frac{d d_{1}}{d x}
$$

which is (A.1.8). The upper layer at the weir satisfies

$$
0=\frac{q_{U}}{\rho_{0}} \frac{d p_{s}}{d x}+q_{\Delta U} \frac{d d_{1}}{d x}+\frac{q_{U}^{3}}{d_{2}^{3}} S_{E U} \frac{d d_{1}}{d x}-q_{\Delta U} S_{P U} \frac{d d_{1}}{d x}
$$

which is (A.2.5) with $F_{2}^{2}=\frac{q_{U}^{3}}{h_{U}^{3} q_{\Delta U}}$. These two equations can be rearranged to give

$$
\frac{d p_{s}}{d x}=\frac{\rho_{0}}{q_{U}}\left(-q_{\Delta U} \frac{d d_{1}}{d x}-\frac{q_{U}^{3}}{d_{2}^{3}} S_{E U} \frac{d d_{1}}{d x}+q_{\Delta U} S_{P U} \frac{d d_{1}}{d x}\right)
$$

and

$$
\frac{d p_{s}}{d x}=\frac{\rho_{0}}{q_{U}}\left(q_{\Delta U} S_{P U}-q_{\Delta U}-\frac{q_{U}^{3}}{\left(D-d_{1}-h_{b}\right)^{3}} S_{E U}\right) \frac{d d_{1}}{d x}
$$

and then combined to eliminate the surface pressure, giving

$$
0=\frac{q_{L}}{\rho_{0}} \frac{\rho_{0}}{q_{U}}\left(q_{\Delta U} S_{P U}-q_{\Delta U}-\frac{q_{U}^{3}}{\left(D-d_{1}-h_{b}\right)^{3}} S_{E U}\right) \frac{d d_{1}}{d x}-2 \frac{q_{L}^{3}}{d_{1}^{3}} \frac{S_{E}}{2} \frac{d d_{1}}{d x}+q_{\Delta} S_{P} \frac{d d_{1}}{d x}
$$

Flow is critical at the crest of the weir, so $\frac{d d_{1}}{d x} \neq 0$ and can be cancelled, resulting in

$$
0=\frac{q_{L}}{q_{U}}\left(q_{\Delta U} S_{P U}-q_{\Delta U}-\frac{q_{U}^{3}}{\left(D-d_{1}-h_{b}\right)^{3}} S_{E U}\right)-2 \frac{q_{L}^{3}}{d_{1}^{3}} \frac{S_{E}}{2}+q_{\Delta} S_{P}
$$


In the two-layer approximated (and for 2-layer predictions), the upper layer always has the minimum density in the system, throughout the entire upper layer. Therefore, $q_{\Delta U}=0$. Applying this to (A.3.1) gives

$$
0=\frac{q_{U}^{2} S_{E U}}{\left(D-d_{1}-h_{b}\right)^{3}}+\frac{q_{L}^{2}}{d_{1}^{3}} S_{E}-\frac{q_{\Delta}}{q_{L}} S_{P}
$$

Solve (A.3.2) for $h_{1 e}$ by assuming $q_{e f r a c}$ and using the following definitions:

$$
\begin{gathered}
q_{L}=u_{1 a} h_{1 a}\left(1+q_{e f r a c}\right), \\
q_{U}=u_{2 a} h_{2 a}-u_{1 a} h_{1 a} q_{e f r a c}, \\
q_{\Delta}=\frac{g}{\rho_{0}} \int \Delta \rho u d y=\frac{g}{\rho_{2}}\left(\rho_{1 b}-\rho_{2}\right) u_{1 a} h_{1 a}\left(1+q_{e f r a c}\right)=g^{\prime} u_{1 a} h_{1 a}, \\
\frac{q_{L}}{q_{\Delta}}=\frac{1+q_{e f r a c}}{g^{\prime}} .
\end{gathered}
$$

\section{A.4 Combining upper and lower layer equations to find $h_{1 b}$, knowing $h_{1 e}$}

To calculate $h_{1 b}$ knowing $h_{1 e}$ from the above section, use A.1.7:

$$
\left[q_{L} \frac{p_{s}}{\rho_{0}}+h_{b} q_{\Delta}+\frac{q_{L}^{3}}{d_{1}^{2}} \frac{S_{E}}{2}+q_{\Delta} d_{1} S_{P}\right]_{b}^{e}=0
$$

and A.2.3:

$$
\left[q_{U} \frac{p_{s}}{\rho_{0}}+\left(h_{b}+d_{1}\right) q_{\Delta U}+\frac{q_{U}^{3}}{d_{2}^{2}} \frac{S_{E U}}{2}+q_{\Delta U} d_{2} S_{P U}\right]_{b}^{e}=0 .
$$

Again, since the flow is two-layered, $q_{\Delta U}=0$, so these equations can be simplified to

$$
\left[q_{U} \frac{p_{s}}{\rho_{0}}+\frac{q_{U}^{3}}{d_{2}^{2}} \frac{S_{E U}}{2}\right]_{b}^{e}=0
$$


and

$$
\left[\frac{p_{s}}{\rho_{0}}\right]_{b}^{e}=\left[\frac{q_{U}^{2}}{d_{2}^{2}} \frac{S_{E U}}{2}\right]_{e}^{b}
$$

Substituting them into A.1.7 gives

$$
q_{L}\left[\frac{q_{U}^{2}}{d_{2}^{2}} \frac{S_{E U}}{2}\right]_{e}^{b}+\left[h_{b} q_{\Delta}+\frac{q_{L}^{3}}{d_{1}^{2}} \frac{S_{E}}{2}+q_{\Delta} d_{1} S_{P}\right]_{b}^{e}=0
$$

which can be rearranged to

$$
\left[\frac{q_{U}^{2}}{\left(D-h_{b}-d_{1}\right)^{2}} \frac{S_{E U}}{2}\right]_{e}^{b}+\left[h_{b} \frac{q_{\Delta}}{q_{L}}+\frac{q_{L}^{2}}{d_{1}^{2}} \frac{S_{E}}{2}+\frac{q_{\Delta}}{q_{L}} d_{1} S_{P}\right]_{b}^{e}=0
$$

or

$\frac{q_{U}^{2} S_{E U}}{2}\left(\frac{1}{\left(D-h_{1 b}\right)^{2}}-\frac{1}{\left(D-h_{b}-h_{1 e}\right)^{2}}\right)+h_{b} \frac{q_{\Delta}}{q_{L}}+\frac{q_{L}^{2} S_{E}}{2}\left(\frac{1}{h_{1 e}^{2}}-\frac{1}{h_{1 b}^{2}}\right)+\frac{q_{\Delta}}{q_{L}} S_{P}\left(h_{1 e}-h_{1 b}\right)=0$.

Knowing $h_{q e}$, (A.4.1) can be solved for $h_{1 b}$. The solution for $h_{1 b}$ must also be a jump solution to the WS theory with the assumed entrainment for the given the upstream conditions. Therefore, iteration is required. In each iteration, the entrainment assumed when solving (A.3.2) must be adjusted, a new value for $h_{1 e}$ must be found using (A.3.2), and then a new value of $h_{1 b}$ should be calculated using (A.4.1). If this value of $h_{1 b}$ is not a jump solution, another iteration is required. 


\section{Appendix B}

\section{Taylor-Goldstein equation and solution}

The Taylor-Goldstein equation is used to calculate the internal wave speeds of the numerical simulations, using the continuous velocity and density profiles. The TaylorGoldstein equation is

$$
(U-c)\left(\frac{\partial^{2}}{\partial z^{2}}-k^{2}\right) w-U_{z z} w+\frac{N^{2}}{U-c} w=0
$$

where $w=w(z)$ is the amplitude of the assumed form of the vertical velocity component of the wave solution, $W=w(z) e^{i k(x-c t)}$ (Kundu and Cohen, 2008).

In the long-wave limit, which gives the fastest moving waves in each direction, $k \rightarrow 0$, giving

$$
(U-c) \frac{\partial^{2} w}{\partial z^{2}}-U_{z} z w+\frac{N^{2}}{U-c} w=0
$$

This is solved in matlab using the polyeig routine, which solved an eigenvalue problem in the form

$$
\left(A_{0}+A_{1} \lambda+A_{2} \lambda^{2}+\ldots\right) x=0 .
$$

In equation B.0.2, $c$ is the eigenvalue, and $\psi$ can be discretized using

$$
\frac{\partial^{2} w}{\partial z^{2}}=\frac{1}{\Delta z^{2}}\left(w_{i+1}-2 w_{i}+w_{i-1}\right)
$$


Putting equation B.0.2 in this form of B.0.3 using this discretization gives

$$
\left[U^{2} M+N^{2}-U U_{z z}+c\left(U_{z z}-2 M\right)+c^{2} M\right] w=0
$$

where

$$
M=\frac{1}{\Delta z^{2}}\left(\begin{array}{ccccc}
-2 & 1 & \ldots & & 0 \\
1 & -2 & 1 & \ldots & \\
& 1 & -2 & 1 & \ldots \\
0 & \ldots & & \ddots &
\end{array}\right)
$$

Equation B.0.4 is subject to the boundary conditions $w=0$ at $z=0$ and $z=D$ due to the flat bottom and rigid lid. 


\section{Bibliography}

Afanasyev, Y. and Peltier, W. (2001). On breaking internal waves over the sill in Knight Inlet. Proc. R. Soc. Lond. A, 457:2799-2825.

Alford, M. H., Girton, J., Voet, G., Carter, G., Mickett, J., and Klymak, J. (2013). Turbulent mixing and hydraulic control of abyssal water in the samoan passage. Geophysical Research Letters, 40:4668âĂŞ4674.

Almgren, A., Bell, J., Colella, P., Howell, L., and Welcome, M. (1998). A conservative adaptive projection method for the variable density incompressible Navier-Stokes equations. Journal of Computational Physics, 142:1-46.

Armi, L. and Farmer, D. (2002). Stratified flow over topography: bifurcation fronts and transition to the uncontrolled state. Proceedings of the Royal Society A, 458(2019):513-538.

Aspden, A., Nikiforakis, N., Dalziel, S., and Bell, J. (2008). Analysis of implicit LES methods. Comm. App. Math. and Comp. Sci., 3(1):103-126.

Baines, P. (1995). Topographic Effects in Stratified Flows. Cambridge University Press.

Benjamin, T. (1968). Gravity currents and related phenomenon. J. Fluid Mech., $31: 209-248$.

Borden, Z. and Meiburg, E. (2013a). Circulation based models for Boussinesq gravity currents. Physics of Fluids, 25(101301).

Borden, Z. and Meiburg, E. (2013b). Circulation-based models for Boussinesq internal bores. Journal of Fluid Mechanics, 726.

Borden, Z., Meiburg, E., and Constantinescu, G. (2012). Internal bores: an improved model via a detailed analysis of the energy budget. Journal of Fluid Mechanics, 703:279-314.

Burchard, H. and Rennau, H. (2008). Comparative quantification of physically and numerically induced mixing in ocean models. Ocean Modelling, 20:293-311.

Carr, M., King, S., and Dritschel, D. (2012). Instability in internal solitary waves with trapped cores. Physics of Fluids, 24(016601).

Choi, W. (2006). The effect of a background shear current on large amplitude internal solitary waves. Physics of Fluids, 18(036601). 
Cummins, P. (2000). Stratified flow over topography: time-dependent comparisons between model solutions and observations. Dynamics of Atmospheres and Oceans, $33: 43-72$.

Doyle, J., Shapiro, M., Jiang, Q., and Bartels, D. (2005). Large-amplitude mountain wave breaking over Greenland. Journal of the Atmospheric Sciences, 62:3106-3126.

Farmer, D. and Armi, L. (1999). Stratified flow over topography: the role of smallscale entrainment and mixing in flow establishment. Proc. R. Soc. Lon. A, 455:32213258 .

Farmer, D. and Armi, L. (2001). Stratified flow over topography: models versus observations. Proc. R. Soc. Lon. A, 457:2827-2830.

Farmer, D. and Smith, J. D. (1980). Tidal interaction of a stratified flow with a sill in Knight Inlet. Deep-Sea Research, 27A:239-254.

Gregg, M. and Özsoy, E. (1999). Mixing on the Black Sea shelf north of the Bosphorus. Geophysical Research Letters, 26(13):1869-1872.

Gregg, M. and Özsoy, E. (2002). Flow, water mass changes, and hydraulics in the bosphorus. J. of Geophysical Research, 107(C3).

Gregg, M. and Pratt, L. (2010). Flow and hydraulics near the sill of Hood Canal, a strongly sheared, continuously stratified fjord. Journal of Physical Oceanography, 40:1087-1105.

Grimshaw, R., Pelinovsky, E., Talipova, T., and Kurkin, A. (2004). Simulation of the transformation of internal solitary waves on oceanic shelves. Journal of Physical Oceanography, 34:2774-2791.

Grinstein, F., Margolin, L., and Rider, W., editors (2007). Implicit Large Eddy Simulation: Computing Turbulent Fluid Dynamics. Cambridge University Press.

Helfrich, K. (1995). Time-dependent two-layer hydraulic exchange flows. Journal of Physical Oceanography, 25:359-373.

Helfrich, K. R. and White, B. L. (2010). A model for large-amplitude internal solitary waves with trapped cores. Nonlinear Processes in Geophysics, 17(4):303-318.

Hickel, S., Adams, N., and Mansour, N. (2007). Implicit subgrid-scale modeling for large-eddy simulation of passive-scalar mixing. Physics of Fluids, 19(095102).

Holland, D., Rosales, R., Stefanica, D., and Tabak, E. (2002). Internal hydraulic jumps and mixing in two-layer flows. Journal of Fluid Mechanics, 470:63-83.

Itsweire, E., Koseff, J., Briggs, D., and Ferziger, J. (1993). Turbulence in stratified shear flows: Implications for interpreting shear-induced mixing in the ocean. Journal of Physical Oceanography, 23:1508-1522.

Jacobson, T., Milewski, P. A., and Tabak, E. (2008). Mixing closures for conservation laws in stratified flows. Studies in Applied Mathematics, 121:89-116. 
Josberger, E. and Cheng, R. (2005). Modeling tidal circulation and fresh water mixing in Hood Canal, Washington. USGS Modeling Conference, Port Angeles, Washington, November 15-17, 2005. http://wa.water.usgs.gov/projects/hoodcanal/publications.htm.

Klemp, J., Rotunno, R., and Skamarock, W. (1997). On the propagation of internal bores. Journal of Fluid Mechanics, 331:81-106.

Klymak, J. and Gregg, M. (2001). Three-dimensional nature of flow near a sill. Journal of Geophysical Research, 106(C10):22295-22311.

Klymak, J. and Gregg, M. (2003). The role of upstream waves and a downstream density pool in the growth of lee waves: stratified flow over the Knight Inlet Sill. Journal of Physical Oceanography, 33:1446-1461.

Klymak, J. and Gregg, M. (2004). Tidally generated turbulence over the Knight Inlet Sill. Journal of Physical Oceanography, 34(5):1135-1151.

Kundu, P. K. and Cohen, I. M. (2008). Fluid Mechanics, Fourth Edition. Associated Press.

Lamb, K. (2000). Conjugate slows for a three-layer fluid. Physics of Fluids, $12(9): 2169-2185$.

Lamb, K. (2004). On boundary-layer separation and internal wave generation at the Knight Inlet sill. Proc. R. Soc. Lond. A, 460:2305-2337.

Li, M. and Cummins, P. (1998). A note on hydraulic theory of internal bores. Dynamics of Atmospheres and Oceans, 28:1-7.

Lilly, D. (1978). A severe downslope windstorm and aircraft turbulence event induced by a mountain wave. Journal of the Atmospheric Sciences, 35:59-77.

Lin, Y. and Redekopp, L. (2012). Internal wave shocks in continuously stratified flows with velocity shear. European Journal of Mechanics - B/Fluids, 36(0):176 - 186.

Luzzatto-Fegiz, P. and Helfrich, K. R. (2014). Laboratory experiments and simulations for solitary internal waves with trapped cores. Journal of Fluid Mechanics, $757: 354-380$.

Margolin, L., Rider, W., and Grinstein, F. (2006). River plumes as a soumodeling turbulent flow with implicit LES. Journal of Turbulence, 7(15).

McDougall, T. and Barker, R. (2011). Getting started with teos-10 and the gibbs seawater (gsw) oceanographic toolbox. 28pp., SCOR/IAPSO WG127, ISBN 9780-646-55621-5.

Milewski, P. A. and Tabak, E. G. (2015). Conservation law modelling of entrainment in layered hydrostatic flows. Journal of Fluid Mechanics, 772:272-294.

Nash, J. and Moum, J. (2005). River plumes as a source of large-amplitude internal waves in the coastal ocean. Nature, 437:400-403. 
Nasr-Azadani, M. and Meiburg, E. (2016). Gravity currents propagating into ambients with arbitrary shear and density stratification: vorticity-based modelling. Quarterly Journal of the Royal Meteorological Society.

Ogden, K. A. and Helfrich, K. R. (2016). Internal hydraulic jumps in two-layer flows with upstream shear. Journal of Fluid Mechanics, 789:64-92.

Peltier, W. and Caulfield, C. (2003). Mixing efficiency in stratified shear flows. Annual Review of Fluid Mechanics, 35:135-167.

Peltier, W. and Clark, T. (1979). The evolution and stability of finite-amplitude mountain waves. part II: Surface wave frag and severe downslope windstorms. Journal of the Atmospheric Sciences, 36:1498-1529.

Polzin, K., Speer, K., Toole, J., and Schmitt, R. (1996). Intense mixing of Antarctic Bottom Water in the equatorial Atlantic Ocean. Nature, 380(7):54-57.

Popinet, S. (2003). Gerris: a tree-based adaptive solver for the incompressible Euler equations in complex geometries. Journal of Computational Physics, 190:572-600.

Pratt, L. and Whitehead, J. (2007). Rotating Hydraulics. Springer.

Pratt, L. J., Riemenschneider, U., and Helfrich, K. R. (2007). A transverse hydraulic jump in a model of the faroe bank channel outflow. Ocean Modelling, 19:1-9.

Regev, A. and Hassid, S. (2010). On the streamwise development of density jumps. Journal of Fluids Engineering, 132(2).

Remmler, S. and Hickel, S. (2012). Direct and large eddy simulation of stratified turbulence. Intl. J. Heat Fluid Flow, 35:13-24.

Rohr, J., Itsweire, E., Helland, K., and van Atta, C. (1988). Growth and decay of turbulence in a stably stratified shear flow. Journal of Fluid Mechanics, 195:77-111.

Rottman, J. and Simpson, J. (1989). The formation of internal bores in the atmosphere: A laboratory model. Q.J.R. Meteorol. Soc., 115:941-963.

Sánchez-Garrido, J., Sannino, G., Liberti, L., Lafuente, J. G., and Pratt, L. (2011). Numerical modeling three-dimensional stratified tidal flow over Camarinal sill, Strait of Gibraltar. Journal of Geophysical Research, 116.

Scotti, A., Beardsley, R., and Butman, B. (2007). Generation and propagation of nonlinear internal waves in Massachusetts Bay. Journal of Geophysical Research, 112.

Shaw, W. J., Trowbridge, J., and III, A. W. (2001). Budgets of turbulent kinetic energy and scalar variance in the continental sheld bottom boundary layer. Journal of Geophysical Research, 106(C5):9551-9564.

Smith, R. B. (1985). On severe downslope winds. American Meteorological Society, 42(23):2597-2603. 
Stashchuk, N. and Vlasenko, V. (2007). Numerical modelling of stratified tidal flow over a fjord sill. Ocean Dynamics, 57:325-338.

Stastna, M. and Lamb, K. (2002). Large fully nonlinear internal solitary waves: the effect of background current. Physics of Fluids, 14(9):2987-2999.

St.Laurent, L. and Thurnherr, A. (2007). Intense mixing of lower thermocline water on the crest of the Mid-Atlantic Ridge. Nature, 448(9):680-683.

Thorpe, S. (2010). Turbulent hydraulic jumps in a stratified shear flow. Journal of Fluid Mechanics, 654:305-350.

Ünlüata, U., Aubrey, D., Belberoz, Z., Bologa, A., Eremeev, V., and Vinogradov, M. (1993). International program investigates the Black Sea. EOS. Transactions, American Geophysical Union, 74(36):401-416.

Waite, M. and Smolarkiewicz, P. (2008). Instability and breakdown of a vertical vortex pair in a strongly stratified fluid. J. Fluid. Mech., 606:239-273.

Wesson, J. and Gregg, M. (1994). Mixing at camarinal sill in the strait of gibraltar. Journal of Geophysical Research.

White, B. and Helfrich, K. (2013). Rapid gravitational adjustment of horizontal shear flows. Journal of Fluids Mechanics, 721:86-117.

White, B. and Helfrich, K. (2014). A model for internal bores in continuous stratification. Journal of Fluids Mechanics, 761:282-304.

Wilkinson, D. and Wood, I. (1971). A rapidly varying flow phenomenon a in two-layer flow. Journal of Fluids Mechanics, 47(2):241-256.

Winters, K. (2016). The turbulent transition of a supercritical downslope flow: sensitivity to downstream conditions. Journal of Fluids Mechanics, 792:997-1012.

Winters, K. and Armi, L. (2012). Hydraulic control of continuously stratified flow over an obstacle. Journal of Fluids Mechanics, 700:502-513.

Winters, K. and Armi, L. (2013). The response of a continuously stratified fluid to an oscillating flow past an obstacle. Journal of Fluids Mechanics, 727:83-118.

Wood, I. and Simpson, J. (1984). Jumps in layered miscible fluids. Journal of Fluids Mechanics, 140:329-342.

Wyngaard, J. and Coté, O. (1971). The budgets of turbulent kinetic energy and temperature variance in the atmospheric surface layer. Journal of the Atmospheric Sciences, 28:190-201.

Wyngaard, J. C. (2010). Turbulence in the Atmosphere. Cambridge University Press.

Zhou, Y., Grinstein, F., Wachtor, A., and Haines, B. (2014). Estimating the effective reynolds number in implicit large-eddy simulation. Phys. Rev. E, 89:013303. 